\author{
Universidade de São Paulo \\ Instituto de Física
}

\title{
Estrutura Eletrônica de Impurezas Simples e Complexas Envolvendo Átomos Leves em GaAs
}

\author{
Walter Manuel Orellana Muñoz
}

Tese apresentada no Instituto
de Física da USP, para
a obtenção do título
de doutor em ciências.

Orientador:

Prof. Dr. Armando Corbani Ferraz

Banca examinadora:

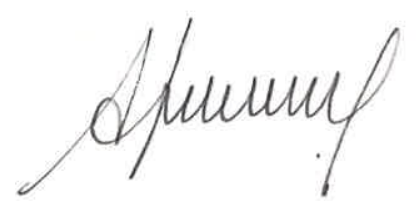

Prof. Dr. Adalberto Fazzio - USP

Prof. Dr. Alex Antonelli - UNICAMP

Prof. Dr. Klaus Krambrock - UFMG

Prof, Dr. Nelson Studart - USPCar
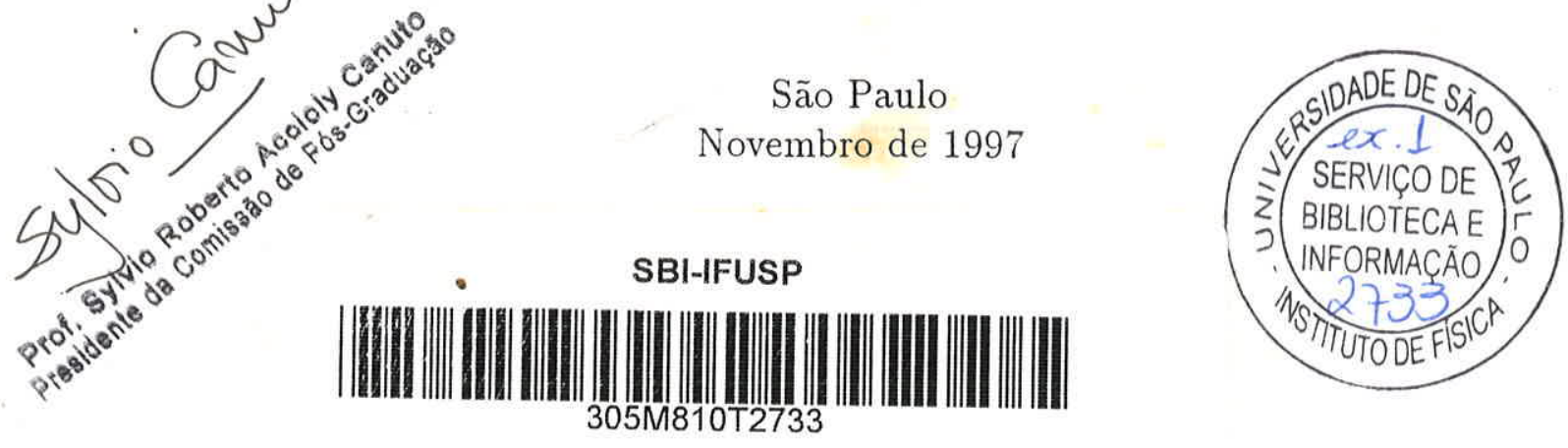


\section{5 \\ 19971 e}

FICHA CATALOGRÁFICA

Preparada pelo Serviço de Biblioteca e Informação

do Instituto de Física da Universidade de São Paulo

Muñoz, Walter Manuel Orellana

Estrutura Eletrônica de Impurezas Simples e Complexas Envolvendo Átomos Leves em GaAs. São Paulo, 1997.

Tese (Doutoramento) Universidade de São Paulo. Instituto de Física - Departamento de Física dos Materiais e Mecânica.

Área de Concentração: Física da Matéria Condensada Orientador: Prof. Dr. Armando Corbani Ferraz

Unitermos: 1. Oxigênio; 2. Nitrogênio; 3. Hidrogênio; 4. Pseudopotencial; 5. Estrutura Eletrônica. 


\section{Agradecimentos}

Ao Prof. Armando Corbani Ferraz pela orientação e por ter me oferecido a oportunidade de trabalhar com métodos de cálculo sofisticados.

Aos Profs. Adalberto Fazzio e Lucy Assali pelo interesse e discussões relacionados com este trabalho.

Aos colegas e amigos: Anderson, Claudino, Douglas, Hiroki, Pedro, Rogério e Tomé, pela agradável convivência durante estes anos; e um agradecimento especial ao Hiroki pelas dicas e sugestões relacionadas ao método e pela leitura crítica da tese.

Ao colega J. Kintop pela ajuda computacional.

Aos centros de computação: LCCA da Universidade de São Paulo, CENAPAD de Campinas e LNCC do Rio de Janeiro.

Às agências CNPq e FAPESP pela ajuda financeira.

E finalmente à minha familia pelo apoio nas minhas decisões. 


\section{Índice}

$\begin{array}{ll}\text { Abstract } & 3\end{array}$

Resumo 4

Introdução $\quad 5$

1 Método de Cálculo 9

1.1 Teoria do Funcional da Densidade . . . . . . . . . . . . . 10

1.1.1 Aproximação da Densidade Local (LDA) . . . . . . . . 14

1.2 Teoria do Pseudopotencial . . . . . . . . . . . . . . 16

1.2.1 Pseudopotenciais Separáveis . . . . . . . . . . . . 21

1.2.2 Pseudopotencial de Troullier-Martins . . . . . . . . . 23

1.3 Energia Total no Espaço dos Momento . . . . . . . . . . . 25

1.3.1 Pontos Especiais na Zona de Brillouin . . . . . . . . 34

1.3.2 Forças de Hellmann-Feynman . . . . . . . . . . . 37

1.4 Dinâmica Molecular de Car-Parrinello . . . . . . . . . . . . . 39

2 Impurezas Substitucionais de Oxigênio e Nitrogênio em GaAs 43

2.1 Oxigênio Substitucional em GaAs . . . . . . . . . . . 44

2.1.1 Oxigênio Substituindo um Arsênio em GaAs . . . . . . 45

2.2 Nitrogênio Substitucional em GaAs . . . . . . . . . . . 56

2.2.1 Nitrogênio Substituindo um Arsênio em GaAs . . . . . 57

2.2.2 Nitrogênio Substituindo um Gálio em GaAs . . . . . . 62

3 Impurezas Complexas $\mathrm{O}-\mathrm{H}$ e $\mathrm{N}-\mathrm{H}$ em $\mathrm{GaAs} \quad 73$

3.1 Complexo $O_{A s}-H$ em $G a A s \ldots \ldots \ldots \ldots . \ldots . \ldots 75$

3.2 Complexo $N_{A s}-H$ em $G a A s \ldots \ldots \ldots \ldots$

3.3 Complexo $N_{G a}-H$ em GaAs . . . . . . . . . . . . 90 
4 Complexos de Nitrogênio e Pares de Hidrogênios em $G a A s \quad 99$ 4.1 Complexo $N_{A s}-H_{2}$ em GaAs . . . . . . . . . . . . 102

4.2 Complexo $N_{G a}-H_{2}$ em $G a A s \ldots \ldots \ldots \ldots 11$

5 Conclusões 119

A. Geração dos Pseudopotenciais 124

B Parâmetros Estructurais do GaAs $\quad 129$

C Testes de Dispersão e Convergência 134

$\begin{array}{ll}\text { D Energia de Formação } & 137\end{array}$ 


\section{Abstract}

We report first-principles calculations of the electronic structure, atomic geometry and formation energy for the isolated oxygen and nitrogen substitutional impurities in $G a A s\left(O_{A s}, N_{A s}\right.$ and $\left.N_{G a}\right)$. Also we performed electronic structure and atomic geometry calculations for the neutral complexes formed by the same substitutional impurities and interstitial hydrogen atoms $\left(\mathrm{O}_{A s}-H\right.$, $\mathrm{N}_{A s}-\mathrm{H}, \mathrm{N}_{G a}-\mathrm{H}, \mathrm{N}_{A s}-\mathrm{H}_{2}$ and $\mathrm{N}_{G a^{-}}-\mathrm{H}_{2}$ ).

Our results for the $O_{A s}$ and $N_{G a}$ centers for different charge states show Jahn-Teller distortions which induce unstable charge states, implying in a negative- $\mathrm{U}$ behaviour for each center. The $N_{A s}$ center remains on-site for all the charge states studied. All the substitutional impurity give rise deep levels in the gap.

For the $O-H$ and $N-H$ complexes we found several metastable configurations related to different hydrogen equilibrium positions, with energies ranging from 0.5 to $2.5 \mathrm{eV}$ relative to the stable configuration. The stable configuration for the $O_{A s}-H$ complex shows a weak interaction between oxygen and hydrogen, while for the $N_{A s}-H$ and $N_{G a}-H$ complexes it shows the formation of a $\mathrm{NH}$ dimer which is bonded to the lattice.

For the $\mathrm{N}-\mathrm{H}_{2}$ complexes we also found several metastable configuration. The stable configuration for the $N_{A s}-H_{2}$ complex shows one $H$ atom forming a $N H$ dimer with nitrogen, while the second one bonds with a nearestneighbour $\mathrm{Ga}$ atom, in $\mathrm{C}_{3}$, symmetry. For the $\mathrm{N}_{\mathrm{Ga}}-\mathrm{H}_{2}$ complex we observed the formation of a $\mathrm{NH}_{2}$-like molecule also bonded to the lattice.

The passivation and activation properties related to hydrogen atom and their interaction with the gap levels are discussed. 


\section{Resumo}

Apresentamos cálculos de primeiros princípios da geometria atômica, energia de formação e estrutura eletrônica para as impurezas substitucionais de oxigênio e nitrogênio em GaAs $\left(O_{A s}, N_{A s}\right.$ e $\left.N_{G a}\right)$. Também estudamos a geometria atômica e estrutura eletrônica dos complexos neutros formados pelas mesmas impurezas substitucionais e átomos de hidrogênio intersticial $\left(O_{A s}-H\right.$, $\mathrm{N}_{A s}-\mathrm{H}, \mathrm{N}_{G a^{-}}-\mathrm{H}, \mathrm{N}_{A s}-\mathrm{H}_{2}$ e $\left.\mathrm{N}_{G a}-\mathrm{H}_{2}\right)$.

Nossos resultados para os centros $O_{A s}$ e $N_{G a}$, em diferentes estados de carga, mostram distorções Jahn-Teller as quais induzem estados de carga não estáveis, observando-se um comportamento U-negativo para cada centro. Entretanto para o centro $N_{A s}$ não foram observadas distorções. Em todos os sistemas estudados, as impurezas introduzem níveis profundos no gap.

Para os complexos $\mathrm{O}-\mathrm{H}$ e $\mathrm{N}-\mathrm{H}$ foram encontradas várias configurações metaestáveis, correspondentes a diferentes posições de equilíbrio do átomo de hidrogênio, as quais apresentam energias entre $0.5 \mathrm{e} 2.5 \mathrm{eV}$ relativas à configuração estável. Na configuração estável do complexo $O_{A s}-H$, oxigênio não interage diretamente com hidrogênio, ligando-se a três gálios primeiros vizinhos. Entretanto para os complexos $N_{A s}-H$ e $N_{G a}-H$ é observada a formação de um dímero $N H$ ligado à rede.

Para os complexos $\mathrm{N}-\mathrm{H}_{2}$ também são encontradas várias configurações metaestáveis. O complexo $N_{A s}-H_{2}$ apresenta uma configuraçāo estável onde um dos hidrogênios forma o dímero $N H$, enquanto que o segundo fica ligado a um gálio primeiro vizinho, em simetria $C_{3 v}$. Para o complexo $\mathrm{NGa}_{G a}-\mathrm{H}_{2}$ é observada a formação de uma molécula do tipo $\mathrm{NH}_{2}$, a qual também se liga à rede.

As propriedades passivadora e ativadora do átomo de hidrogênio, como também sua interação com os níveis no gap, são discutidas para cada complexo. 


\section{Introdução}

A compreensão das propriedades eletrônicas e estruturais de centros profundos em semicondutores tem atraido a atenção dos pesquisadores ininterruptamente durante os últimos 25 anos. Centros profundos são defeitos da rede ou impurezas que, ao invés das impurezas rasas, provocam perturbaçōes locais severas, introduzindo estados ligados na região central da banda proibida dos semicondutores, sendo referidos comumente como níveis profundos. As funções de onda associadas a estes estados ligados são extremadamente localizadas abrangendo distâncias da ordem de um parâmetro de rede em torno do defeito. Os centros profundos alteram drasticamente os processos de difusão de portadores que no caso de dispositivos constitui um problema dramático, e devido a sua interação ser forte e de curto alcance, reagem facilmente com outras impurezas provocando defeitos estendidos, chegando até a modificar as características do material (formação de cluster, oxidação etc.)

Entre os centros profundos mais estudados encontram-se a vacância em silício, o centro conhecido como EL2 em $G a A s$ e a impureza de oxigênio em silício, $G a P$ e $G a A s$. Estes centros têm cativado a imaginação dos grupos teóricos da área de defeitos, incentivando-os a desenvolver técnicas cada vez mais sofisticadas as quais têm confirmado e também contrariado uma parte importante do grande volume de informações experimentais disponíveis. $O$ primeiro estudo teórico bem sucedido da vacância em silício é relativamente recente (1978), sendo obtido por Bernholc et al. ${ }^{1,2}$ e Baraff e Schlüter, ${ }^{3,4}$ separadamente, utilizando o método da função de Green autoconsistente. Neste pela primeira vez foram obtidas energias de uma partícula e funções de onda para a vacância não relaxada, as quais permitiram estimativas realísticas da posição dos níveis de ocupação ou estados de transição. Posteriormente, nos anos oitenta, outro centro profundo agora em $G a A s$ passou a ser o mais estudado, trata-se do centro EL2. Este é caracterizado por um nível doador localizado no meio do gap, onde o mais surpreendente é a existência do nível profundo em um estado metaestável originado por uma grande relaxação da rede. Originalmente este centro foi relacionado a uma impureza de oxigênio, 
sendo posteriormente descartado o envolvimento desta ou de qualquer outra impureza. Na atualidade a hipótese mais aceita é a existência de um defeito da rede relacionado a um antisítio de arsênio. Este centro têm sido estudado com diversos métodos teóricos, inclusive de primeiros princípios, os quais entretanto não têm conseguido conciliar todos os resultados experimentais disponíveis, mostrando que este continua sendo um problema em aberto. Por outro lado a impureza de oxigênio em semicondutores elementares e compostos é outro dos centros profundos amplamente estudados do ponto de vista experimental. É sabido que quando ocupa um sítio substitucional em silício sofre um deslocamento espontâneo na direção $\langle 100\rangle$, formando uma ligação do tipo Si-O-Si. ${ }^{5}$ Este deslocamento ocorre no estado de carga neutro onde o estado fundamenal não tem degenerecência orbital. Oxigênio em $G a P$ quando ocupa um sítio de fósforo $\left(O_{P}\right)$, forma um centro profundo eletricamente ativo, tendo-se sugerido ${ }^{6}$ um estado metaestável para este centro no estado de carga negativo, mas nada tem sido totalmente definido. Mais recentemente, estudos experimentais de oxigênio substitucional em GaAs quando ocupa um sítio de arsênio $\left(O_{A s}\right)$, também tem mostrado deslocamentos importantes da impureza, onde é observada a formação da ligação $G a-O-G a^{7}$ de forma similar ao silício. Os estados de carga onde ocorreriam estes deslocamentos ainda são motivo de controvérsia.

Cálculos teóricos de primeiros princípios, envolvendo a impureza de oxigênio em semicondutores considerando-se a relaxação da rede, são escasos. As principais dificuldades encontram-se na correta descrição dos orbitais atômicos do oxigênio e o número adequado de átomos que devem ser considerados, seja em cálculos de supercélula ou clusters, o que faz ser necessário um enorme esforço computacional. Um grande avanço neste sentido foi introduzido por Car e Parrinello ${ }^{8}$ em 1985, propondo um método alternativo para o cálculo da estrutura eletrônica e geometria atômica dentro da teoria do funcional da densidade ${ }^{9}$ (DFT). Neste método, os elétrons e núcleos são tratados simultaneamente em um esquema de dinâmica molecular de modo que a parte eletrônica e a relaxação da rede são obtidas diretamente. Esta nova técnica tem permitido a realização de cálculos com um número maior de átomos na supercélula ou com um número maior de elementos no conjunto base, dentro de um requerimento computacional acessível. Uma outra vantagem tem sido o desenvolvimento de uma nova geração de pseudopotencias chamados "suaves" que se caracterizam por descrever orbitais atômicos profundos, tais como os orbitais $p$ dos elementos da primeira linha da Tabela Periódica, precisando de uma expansão em ondas planas menor que com os pseudopotenciais convencionais. 
A investigação de impurezas leves em $G a A s$, tais como carbono, nitrogênio e oxigênio, é de particular interesse para o desenvolvimento e otimização da tecnologia de crescimento de cristais. Este tipo impurezas constituem os principais contaminantes em praticamente todos os semicondutores, estando presentes nos próprios ambientes de crescimento, na forma de gases, ou em posteriores tratamentos químicos a que os dispositivos são submetidos, principalmente na forma de solventes. Estes átomos são altamente reativos, e quando incorporados substitucionalmente na estrutura cristalina de semicondutores, interagem fortemente com impurezas intersticiais, em particular com átomos de hidrogênio. Recentes experiências de modos locais de vibração (LVM) têm detectado frequiências na região do infravermelho as quais têm sido atribuidas aos modos normais dos pares $\mathrm{C}-\mathrm{H}, \mathrm{O}-\mathrm{H}$ e $\mathrm{N}-\mathrm{H}$ em $\mathrm{GaAs}{ }^{1-14}$ e $\mathrm{N}-\mathrm{H}_{2}$ em $\mathrm{GaP}$ e $G a A s .{ }^{15,16}$ Esta atividade óptica é esperada considerando-se que o hidrogênio é a impureza mais comum em todos os semicondutores, possuindo uma grande afinidade eletrônica. Entretanto hidrogênio também tem sido introduzido deliberadamente nos semicondutores, devido a sua conhecida propriedade passivadora em centros eletricamente ativos, cujo principal efeito é o deslocamento dos níveis de impureza fora da banda proibida. Também é sabido seu efeito contrário, isto é, a ativação elétrica de centros inativos, introduzindo níveis no gap. Entretanto nada é sabido sobre sua estrutura microscópica especialmente na sua interação com centros profundos.

Apesar do grande esforço teórico e experimental dedicado ao estudo do hidrogênio em semicondutores cristalinos, especialmente em silício, ainda permanecem inexplicados uma grande quantidade de fenômenos relacionados a esta impureza. Entre estas podemos citar o mecanismo de passivação de centros dopantes multivalentes; a "reativação" de centros previamente passivados, i.e., a reversibilidade entre centros passivados/ativados; a influência do hidrogênio na difusão de outras impurezas tais como oxigênio em silício; ${ }^{17} \mathrm{com}$ plexos envolvendo defeitos ou impurezas interagindo com mais de um átomo de hidrogênio, etc. Algumas destas questões aqui apresentadas sāo discutidas neste trabalho.

A motivaçāo deste trabalho é apresentar um cálculo de primeiros princípios utilizando os métodos do estado da arte para as impurezas substitucionais de oxigênio e nitrogênio e sua interação com a impureza intersticial de hidrogênio, que constituem os átomos mais abundantes nas diversas técnicas de crescimento de cristais e processamento de dispositivos. A importância das impurezas de oxigênio e nitrogênio é fundamental na tecnologia de semicondutores visto que estas introduzem níveis profundos na banda proibida, são altamente reativas e muito fáceis de serem incorporadas na estrutura cristalina. 
Seu tratamento teórico tem permanecido proibitivo até muito pouco tempo atrás e somente agora com o desenvolvimento de métodos de cálculo eficientes e de computadores de grande porte, tem sido possível uma descrição mais rigorosa.

A organização da tese é feita da seguinte maneira: no Capítulo 1 apresentamos o formalismo teórico utilizado no estudo dos sistemas de impureza em GaAs. No Capítulo 2 mostramos os resultados dos cálculos da geometria atômica de equilíbrio, estrutura eletrônica e energia de formação para as impurezas substitucionais de oxigênio substituindo um arsênio $\left(O_{A s}\right)$, nitrogênio substituindo um arsênio $\left(N_{A s}\right)$ e nitrogênio substituindo um gálio $\left(N_{G a}\right)$. No Capítulo 3 apresentamos os cálculos de geometria atômica de equilíbrio e estrutura eletrônica para os complexos formados por oxigênio substitucional e hidrogênio intersticial $\left(O_{A s}-H\right)$, e nitrogênio substitucional e hidrogênio intersticial $\left(N_{A s^{-}}-\mathrm{H}\right.$ e $\left.N_{G a^{-}} H\right)$. No Capítulo 4 apresentamos os resultados da geometria atômica de equilíbrio e estrutura eletrônica para os complexos formados por nitrogênio substitucional e dois hidrogênios intersticiais $\left(\mathrm{N}_{\mathrm{As}}-\mathrm{H}_{2}\right.$ e $N_{G a}-H_{2}$ ). Finalmente no Capítulo 5 apresentamos nossas conclusões. Também incluímos quatro apêndices onde são descritos alguns detalhes técnicos e os principais testes para o método de cálculo. 


\section{Capítulo 1}

\section{Método de Cálculo}

Neste capítulo descreveremos o método utilizado no cálculo teórico da energia total e a subsequente minimização desta com respeito das coordenadas electrônicas e nucleares. A energia total de um sistema de muitas partículas pode ser calculada unicamente fazendo-se um grande número de aproximações, a fim de se diminuir a complexidade do problema. Devido à grande diferença entre as massas dos eletrons e dos núcleos, e a natureza das forças ser a mesma para todas as partículas, a primeira aproximação que surge é considerar o movimento dos elétrons respondendo instantaneamente ao movimento dos núcleos, i.e., reduzindo o problema da dinâmica de elétrons e núcleos a um sistema que considera a dinâmica dos elétrons em uma configuração nuclear congelada. Esta separação entre coordenadas eletrônicas e nucleares na função de onda de muitos corpos é conhecida como aproximação adiabática ou de BornOppenheimer.

Outras simplificaçōes que devem ser feitas estão relacionadas com as interaçōes elétron-elétron e elétron-íon. A interação elétron-elétron é modelada pela teoria do funcional da densidade (DFT) ${ }^{9.10}$ Nela o problema de um gás de elétrons fortemente interagentes, na presença de um potencial externo devido aos núcleos, pode ser representado equivalentemente por um conjunto de sistemas de um elétron movimentando-se em um potencial efetivo. Estes sistemas de um elétron são descritos pelas equações de Kohn-Sham. A simplificação introduzida nesta teoria considera o potencial efetivo como sendo local, devido a um gás de elétrons homogêneo. Esta aproximação conhecida como da densidade local (LDA) ${ }^{18}$ tem dado bons resultados para uma serie de propriedades do estado fundamental em semicondutores, tais como o parâmetro de rede e níveis de energia, no entanto também apresenta erros consideráveis, como por exemplo na largura da banda proibida que tem subestimado seu 
valor. Em alguns casos, a banda proibida chega a ser até $50 \%$ menor que seu valor experimental. Com respeito a interação elétron-íon, uma aproximação bastante bem sucedida é a do método de pseudopotencial. ${ }^{19}$ Neste, o forte potencial entre os elétrons de caroço e núcleos atômicos no cristal não são incluidos explicitamente no cálculo, o único potencial considerado é aquele sentido pelos elétrons de valência, que é devido aos caroços atômicos ou íons. Este potencial mais fraco é chamado de pseudopotencial.

Por último, para modelar o cristal infinito é utilizada a aproximação da supercélula. Esta consiste em uma célula unitaria ampliada, contendo varios parâmetros de rede, a qual é repetida no espaço. Esta célula pode conter defeitos os que não afetam a simetria do sistema, permitindo o uso da simetria translacional da rede e, portanto, o teorema de Bloch. Nestas circunstâncias as funções de onda são expandidas em um conjunto de ondas planas, onde as equações de Kohn-Sham adquirem uma forma matricial. A dimensão da matriz é determinada pela escolha de uma energia de corte que é proporcional a energia cinética eletrônica no espaço recíproco.

O procedimento de cálculo utilizado na obtenção da energia total, consiste na resolução autoconsistente das equações de Kohn-Sham para os elétrons de valência do sistema. Tradicionalmente esta equação é resolvida por métodos de diagonalização de matriz. No entanto utilizando-se a técnica de minimização iterativa introduzida no método de dinâmica molecular de Car e Parrinello, ${ }^{8}$ é possível obter simultaneamente as autofunções de KohnSham e as forças iônicas do sistema sem precisar de diagonalização alguma, permitindo assim um cálculo rápido e eficiente da energia total do sistema. A seguir apresentaremos uma descrição detalhada das diferentes aproximações acima citadas.

Todas as fórmulas utilizadas neste capítulo, serão dadas em unidades atômicas, onde: $\hbar=1, m_{e}=1, e=1, \epsilon_{0}=1 / 4 \pi$. A unidade de energia é o Hartree, $(1$ Hartree $=2$ Rydberg $=27.211652 \mathrm{eV})$.

\subsection{Teoria do Funcional da Densidade}

A teoria do funcional da densidade (DFT), tem o mérito de caracterizar um problema bastante complexo, que é a descrição dos efeitos de troca e correlação em um gás de elétrons interagentes não homogêneo, por meio de um de um conjunto de equações de um elétron. A base desta teoria é constituida 
por dois teoremas, devidos a Hohemberg e Kohn $(1964),{ }^{9}$ cujas demonstrações podem ser encontradas na referência [10].

Teorema 1 A funçâo de onda do estado fundamental, para um sistema de muitos elétrons, $\Psi_{0}$, é um funcional único da densidade de carga eletrônica, $\rho(\mathbf{r})$, i.e., $\Psi_{0}=\Psi_{0}[\rho(\mathbf{r})]$.

O teorema 1 mostra que a densidade de carga eletrônica é uma variável básica e independente para o problema de muitos elétrons no estado fundamental. Ela determina univocamente a função de onda $\Psi_{0}$, e por conseqüência também determina qualquer interação eletrônica do sistema: energia cinetica, potencial externo, etc. Antes de discutir o segundo teorema, vamos fundamentar o formalismo utilizado na descrição do problema.

Consideremos um sistema de $N$ elétrons sujeitos a um potencial externo $V_{\text {ext }}(\mathbf{r})$. Seja $\Psi_{0}\left(\mathrm{r}_{1}, \mathrm{r}_{2}, \ldots, \mathrm{r}_{N}\right)$ a função de onda do estado fundamental (não degenerado) e $\rho(r)$ a densidade de carga eletrônica correspondente. Definimos o funcional energia, associado ao potencial externo no estado fundamental como:

$$
E_{v}\left[V_{e x t}, \rho(\mathbf{r})\right] \equiv \int d \mathbf{r} V_{e x t}(\mathbf{r}) \rho(\mathbf{r})
$$

Como $\rho(\mathbf{r})$ é a variável fundamental do sistema, temos que:

$$
E_{v}\left[V_{e x t}, \rho(\mathbf{r})\right]=E_{v}[\rho(\mathbf{r})]
$$

Para uma densidade de carga arbitrária $n(\mathbf{r})$, obtida a partir de alguma função de onda $\Psi\left(\mathbf{r}_{1}, \mathbf{r}_{2}, \ldots, \mathbf{r}_{N}\right)$ antisimétrica, e considerando unicamente a interação elétron-elétron, definimos o funcional $F[n(\mathrm{r})]$ como:

$$
F[n(\mathbf{r})] \equiv\left\langle\Psi\left|\left(T+V_{e e}\right)\right| \Psi\right\rangle
$$

onde $T$ e $V_{e e}$ são os operadores energia cinética e de interação eletrônica respectivamente. O funcional $F[n]$ é universal, nāo dependendo do número de partículas nem do potencial externo. Logo o funcional energia total para um sistema de elétrons interagentes na presença de um potencial externo $V_{\text {ext }}$, é um funcional único da densidade, e pode ser escrito como:

$$
E_{t}[n(\mathbf{r})]=\int d \mathbf{r} V_{\text {ext }}(\mathbf{r}) n(\mathbf{r})+F[n(\mathbf{r})]
$$


Teorema 2 O funcional $E_{t}[n(\mathbf{r})]$ obedece ao princípio variacional na densidade de carga $n(\mathbf{r})$, isto é:

$$
\frac{\delta E_{t}[n(\mathbf{r})]}{\delta n(\mathbf{r})}=0 \quad \Longleftrightarrow n(\mathbf{r})=\rho(\mathbf{r}) .
$$

O teorema 2 mostra que o funcional $E_{t}[n(\mathbf{r})]$ assume o valor mínimo, ou a energia do estado fundamental, quando a densidade arbitrária $n(\mathbf{r})$ é igual a densidade de carga eletrônica $\rho(\mathbf{r})$, para o mesmo número de elétrons $N$. Se o funcional $F[n]$ fosse uma função conhecida e simples da densidade eletrônica $n$, a determinação da energia e densidade de carga do estado fundamental para um sistema de muitos elétrons se reduziria a um problema simples de minimização.

Das equações (1.3) e (1.4), a energia do estado fundamental de um gás de elétrons interagentes, não homogêneo, na presença de um potencial externo devido aos íons, pode ser escrito como:

$$
E_{t}[\rho]=\int d \mathbf{r} V_{i o n}(\mathbf{r}) \rho(\mathbf{r})+\frac{1}{2} \iint d \mathbf{r} d \mathbf{r}^{\prime} \frac{\rho(\mathbf{r}) \rho\left(\mathbf{r}^{\prime}\right)}{\left|\mathbf{r}-\mathbf{r}^{\prime}\right|}+G[\rho]
$$

onde $G[\rho]$ é outro funcional universal da densidade, introduzido por Kohn e Sham (1965), os quais propuseram uma aproximação para $G[\rho]$ que leva a um procedimento analogo ao método de Hartree, onde agora são incluidas todas as correções ao modelo do elétron independente. Se $G[\rho]$ é dividido convenientemente em duas partes:

$$
G[\rho] \equiv T_{0}[\rho]+E_{x c}[\rho]
$$

onde $T_{0}[\rho]$ é a energia cinética de um sistema de elétrons não interagentes com densidade $\rho(\mathbf{r})$, e $E_{x c}[\rho]$ é um funcional da densidade que representa todas as contribuições devido a um sistema interagente, i.e., os efeitos de troca e correlação $(x c)$. A origem dos termos de troca e correlação vem de um fenômeno puramente quântico. A exigência de antisimetria da função de onda para um sistema de férmions com respeito a troca de rótulo de duas partículas, dá origem a uma inter-relação entre suas componentes espacial e de spin. Se temos dois elétrons de spins paralelos (componente de spin simétrica), sua componente espacial deve ser antisimétrica. A antisimetria da função espacial tem o efeito de uma separação entre os elétrons de mesmo spin, produzindo uma redução na energia coulombiana do sistema que é conhecida como energia de troca. Por outro lado, a diferença de energia entre um sistema real de 
muitos elétrons e a energia obtida considerando-se um sistema de elétrons não interagentes, é a energia de correlaçāo.

Substituindo (1.7) em (1.6), e considerando uma densidade de carga arbitrária $n(\mathbf{r})$, é possível obter uma expresão exata para a energia total de um sistema de elétrons interagentes de densidade $n$, dada por:

$$
E_{t}[n]=T_{0}[n]+\int d \mathbf{r} V_{i o n}(\mathbf{r}) n(\mathbf{r})+\frac{1}{2} \iint d \mathbf{r} d \mathbf{r}^{\prime} \frac{n(\mathbf{r}) n\left(\mathbf{r}^{\prime}\right)}{\left|\mathbf{r}-\mathbf{r}^{\prime}\right|}+E_{x c}[n]
$$

Segundo o teorema 2 , aplicando o princípio variacional na equação $(1.8), E_{t}[n]$ assume o valor do estado fundamental para a densidade correta. Mas antes é necessário ter uma expresão para a energia cinética de um sistema de elétrons não interagentes, $T_{0}[n]$. Consideremos um sistema de $N$ elétrons não interagentes, movendo-se em um potencial $V(\mathbf{r})$ que dá origem a uma densidade de carga $n(\mathbf{r})$ definida por:

$$
n(\mathbf{r}) \equiv \sum_{i=1}^{o c c}\left|\phi_{i}(\mathbf{r})\right|^{2}
$$

onde a soma é feita nos estados ocupados e $\phi_{i}(\mathbf{r})$ são as funçōes de onda dos eletrons não interagentes que satisfazem a equação de Schrödinger de uma partícula:

$$
\left[-\frac{1}{2} \nabla^{2}+V(\mathbf{r})\right] \phi_{i}(\mathbf{r})=\varepsilon_{i} \phi_{i}(\mathbf{r})
$$

fazendo $\varepsilon_{i}=\left\langle\phi_{i}|H| \phi_{i}\right\rangle$ e somando-se sobre todos os estados do sistema obtemos:

$$
\sum_{i} \varepsilon_{i}=T_{0}[n]+\int d \mathbf{r} V(\mathbf{r}) n(\mathbf{r})
$$

Substituindo $T_{0}[n]$ na equação $(1.8)$ obtemos:

$E_{t}[n]=\sum_{i} \varepsilon_{i}-\int d \mathbf{r} V(\mathbf{r}) n(\mathbf{r})+\int d \mathbf{r} V_{i o n}(\mathbf{r}) n(\mathbf{r})+\frac{1}{2} \iint d \mathbf{r} d \mathbf{r}^{\prime} \frac{n(\mathbf{r}) n\left(\mathbf{r}^{\prime}\right)}{\left|\mathbf{r}-\mathbf{r}^{\prime}\right|}+E_{x c}[n]$

$E_{t}[n]$ obedece ao princípio variacional quando: 


$$
\begin{aligned}
V(\mathbf{r}) \rightarrow V_{e f}(\mathbf{r}) & \equiv V_{i o n}(\mathbf{r})+\int d \mathbf{r}^{\prime} \frac{n\left(\mathbf{r}^{\prime}\right)}{\left|\mathbf{r}-\mathbf{r}^{\prime}\right|}+\frac{\delta E_{x c}[n]}{\delta n(\mathbf{r})} \\
& =V_{i o n}(\mathbf{r})+V_{H}(\mathbf{r})+V_{x c}(\mathbf{r})
\end{aligned}
$$

onde agora $n$ é a densidade de carga do estado fundamental $(n \rightarrow \rho)$. $V_{e f}(\mathbf{r})$ é o potencial efetivo de Kohn-Sham, que é definido como sendo a soma entre o potencial externo devido aos íons $\left(V_{i o n}\right)$, o potencial de Hartree $\left(V_{H}\right)$ e o potencial de troca-correlação de um elétron $\left(V_{x c}\right)$. Substituindo $V(\mathbf{r})$ por $V_{c f}(\mathbf{r})$ na equação de Schrödinger de uma partícula (1.10), obtemos um conjunto de equações autoconsistentes para o estado fundamental de um sistema de elétrons interagentes, conhecidas como equações de Kohn-Sham:

$$
\left[-\frac{1}{2} \nabla^{2}+V_{i o n}(\mathbf{r})+V_{H}(\mathbf{r})+V_{x c}(\mathbf{r})\right] \phi_{i}(\mathbf{r})=\varepsilon_{i} \phi_{i}(\mathbf{r})
$$

onde

$$
\rho(\mathbf{r})=\sum_{i=1}^{o c c}\left|\phi_{i}(\mathbf{r})\right|^{2} .
$$

Para resolver a autoconsistência, começa-se com uma densidade de prova $\rho(\mathbf{r})$. Assumindo que o funcional $E_{x c}[\rho]$ é conhecido (como veremos depois), contróise o potencial efetivo $V_{c f}(\mathbf{r})$ a partir da equação (1.13). Substituindo $V_{e f}(\mathrm{r})$ na equação (1.14), são calculados os $N$ autovalores e autofunções de Kohn-Sham $\left(\varepsilon_{i}, \phi_{i}\right)$. A partir de (1.15) é obtida uma nova densidade de carga a qual é comparada com a original. Este ciclo é repetido até a autoconsistência ser atingida.

\subsubsection{Aproximação da Densidade Local (LDA)}

O método mais simples possível para se descrever o termo de trocacorrelação em um sistema de muitos elétrons, é conhecido como a aproximação da densidade local (LDA) proposta por Khon e Sham. ${ }^{18}$ Neste a energia de troca-correlação de um gás de elétrons de densidade $\rho(\mathbf{r})$ no ponto $\mathbf{r}$, é considerada como sendo igual a energia de troca-correlação de um gás de elétrons homogêneo com a mesma densidade. Desta forma $\rho(\mathbf{r})$ varia suavemente, sendo possível aproximar: 


$$
E_{x c}[\rho] \simeq \int d \mathbf{r} \rho(\mathbf{r}) \epsilon_{x c}[\rho(\mathbf{r})]
$$

onde $\epsilon_{x c}[\rho(\mathbf{r})]$ é a energia de troca-correlação por elétron, de um gás homogêneo de densidade $\rho(\mathrm{r})$. O potencial de troca-correlação de uma partícula, $V_{x c}(\mathbf{r})$, deve ser redefinido agora dentro da aproximação local como:

$$
V_{x c}(\mathbf{r})=\frac{d}{d \rho}\left\{\rho(\mathbf{r}) \epsilon_{x c}[\rho(\mathbf{r})]\right\} \equiv \mu_{x c}[\rho(\mathbf{r})]
$$

onde $\mu_{x c}[\rho]$ é a contribuição de troca-correlação do potencial químico de um gás de elétrons homogêneo de densidade $\rho$. Se a densidade do gás homogêneo é definida como:

$$
\rho^{-1}=\frac{4 \pi}{3} r_{s}^{3}
$$

onde $r_{s}$ é o raio de Wigner-Seitz, substituindo (1.18) em (1.17) temos que:

$$
\mu_{x c}=\epsilon_{x c}-\frac{r_{s}}{3} \frac{d \epsilon_{x c}}{d r_{s}} .
$$

Finalmente, utilizando a aproximação LDA é possível expressar a energia total do estado fundamental para um sistema eletrônico, substituindo (1.16) e (1.17) em (1.12) temos que:

$$
E_{t}[\rho]=\sum_{i=1}^{o c c} \varepsilon_{i}-\frac{1}{2} \iint d \mathbf{r} d \mathbf{r}^{\prime} \frac{\rho(\mathbf{r}) \rho\left(\mathbf{r}^{\prime}\right)}{\left|\mathbf{r}-\mathbf{r}^{\prime}\right|}+\int d \mathbf{r}\left\{\epsilon_{x c}[\rho(\mathbf{r})]-\mu_{x c}[\rho(\mathbf{r})]\right\} \rho(\mathbf{r})
$$

A energia de troca correlação $\epsilon_{x c}$, tem sido estimada por uma serie de métodos de interpolação a partir dos limites de alta e baixa densidade onde seu cálculo é possível. Em nosso trabalho adotamos a parametrização feita por Perdew-Zunger ${ }^{20}$ dos resultados obtidos por Ceperley-Alder. ${ }^{21}$ Estes cálculos são baseados em uma simulação Monte Carlo de um gás de elétrons interagentes. Para um gás não polarizado temos que:

$$
\epsilon_{x c}=\epsilon_{x}+\epsilon_{c}
$$

onde $\epsilon_{x}$ é a energia de troca e $\epsilon_{\varepsilon}$ a energia de correlação, parametrizadas em função de $r_{s}$, onde: 


$$
\begin{gathered}
\epsilon_{x}=-\frac{0.4582}{r_{s}}, \\
\epsilon_{c}= \begin{cases}-0.1423 /\left(1+1.0529 \sqrt{r_{s}}+0.3334 r_{s}\right), & r_{s} \geq 1 \\
-0.0480+0.0311 \ln r_{s}-0.0166 r_{s}+0.0020 r_{s} \ln r_{s}, & r_{s}<1 .\end{cases}
\end{gathered}
$$

A principal falha que apresenta a aproximação da densidade local, é não considerar correções à energia de troca-correlação $\epsilon_{x c}$ devido a variações na densidade de carga para distâncias pequenas. Apesar deste caráter local forçado, esta aproximação tem dado bons resultados para as propriedades do estado fundamental de sistemas que se encaixam no modelo do gás de Fermi (elétrons quase-livres).

\subsection{Teoria do Pseudopotencial}

O modelo mais difundido na descrição da interação elétron-íon em um sólido considera o átomo constituído por caroços e elétrons de valência. Os caroços são formados pelos núcleos e elétrons de caroço atômicos, os quais são considerados inertes. Assim os elétrons de valência serão os únicos responsáveis pela formação das ligações atômicas. Considerando que os elétrons do cristal movem-se em potenciais diferentes dependendo de sua posição no sólido, perto dos caroços eles sentem um potencial forte com características atômicas e entre os caroços sentem um potencial mais fraco e aproximadamente constante. Desta forma a função de onda dos elétrons do cristal pode ser construída como uma combinação linear de estados de caroço e ondas planas ortogonais a eles. Esta aproximação chamada de ondas planas ortogonalizadas $(O P W)$ foi introduzida por Herring ${ }^{22}$ (1940). Posteriormente Phillips e Kleinman ${ }^{19}(1959)$ mostraram que é possível obter as funções de onda $O P W$, como sendo a solução de um problema onde um potencial repulsivo $V_{R}$ que cancela a maior parte do forte potencial atrativo devido aos caroços $V_{c}$. Como resultado é obtido um potencial mais fraco ou pseudopotencial $V_{p s}$.

Formalmente, se consideramos uma função de onda real $\psi$, formada por uma parte suave $\phi$ que pode ser expandida em ondas planas, e uma parte de caroço, expandida em orbitais de caroço $\phi_{c}$, assim: 


$$
\psi=\phi+\sum_{c} b_{c} \phi_{c}
$$

Forçando a ortogonalidade entre $\psi$ e os estados de caroço, como na aproximação $O P W$, i.e., $\left\langle\phi_{c} \mid \psi\right\rangle=0=\left\langle\phi_{c} \mid \phi\right\rangle+b_{c}$, temos que:

$$
\psi=\phi-\sum_{c}\left\langle\phi_{c} \mid \phi\right\rangle \phi_{c}
$$

A função de onda $\psi$ deve satisfazer a equação de Schrödinger para a hamiltoniana dos elétrons movendo-se em um potencial atrativo e de longo alcance, devido aos caroços $V_{c}$, isto é:

$$
H \psi=\left[T+V_{c}\right] \psi=\varepsilon \psi
$$

onde $\varepsilon$ é o autovalor de energia correta dos sistema. Substituindo (1.25) em (1.26) obtemos:

$$
H \phi+\sum_{c}\left(\varepsilon-\varepsilon_{c}\right)\left|\phi_{c}\right\rangle\left\langle\phi_{c}\right| \phi=\varepsilon \phi
$$

onde $\varepsilon_{c}$ são os autovalores de energia dos estados de caroço. A soma nos estados de caroço representa um operador atuando na parte suave da função de onda do sistema, e substituindo $H$ da equação (1.26) em (1.27) é possível identificar este operador como sendo um potencial repulsivo e de curto alcance, assim:

$$
\left[T+V_{c}+V_{R}\right] \phi=\varepsilon \phi
$$

onde

$$
V_{R} \equiv \sum_{c}\left(\varepsilon-\varepsilon_{c}\right)\left|\phi_{c}\right\rangle\left\langle\phi_{c}\right|
$$

Definimos o operador $V_{p s}$ como:

$$
V_{p s}=V_{c}+V_{R}
$$

O operador $V_{p s}$ representa um potencial fracamente atrativo que é uma conseqüencia do cancelamento entre o forte potencial atrativo $V_{c}$ e o potencial repulsivo $V_{R}$. Este é conhecido como o teorema de cancelamento de PhillipsKleinman. O operador $V_{p s}$ é definido como sendo o pseudopotencial e $\phi$ a 
pseudofunção de onda. Pode ser mostrado que no formalismo de PhillipsKleinman a pseudofunção de onda $\phi$ não obedece a normalização e nem a unicidade. A falta de unicidade permite que esta seja construída arbitrariamente de muitas formas, isto de alguma forma tem dado certa liberdade na procura de pseudopotenciais computacionalmente eficientes.

As funções de onda de valência $\psi$ oscilam rapidamente nas regiões dos caroços, devido ao forte potencial atrativo nesta região. Estas oscilaçōes mantém a devida ortogonalidade com as funçōes de onda de caroço, exigidas pelo princípio de exclusão. Na prática a construção de um pseudopotencial se inicia considerando-se uma pseudofunção $\phi$ com um comportamento suave e sem oscilações dentro da região do caroço. Para as regiões fora do caroço, $\phi$ deve reproduzir identicamente a verdadeira função de onda de valência. Estas exigências fazem necessário forçar a normalização de $\phi$.

Em geral pseudopotenciais ab initio usados no estudo da estrutura eletrônica e propriedades do estado fundamental de sólidos, são construídos a partir de cálculos atômicos que consideram todos os elétrons (caroço e valência). Estes cálculos são feitos resolvendo-se autoconsistentemente as equações de Kohn-Sham radial, dentro da aproximação LDA, dadas por:

$$
\left[-\frac{1}{2} \frac{d^{2}}{d r^{2}}+\frac{\ell(\ell+1)}{2 r^{2}}+V_{e f}[\rho(r)]\right] r R_{n \ell}(r)=\varepsilon_{n \ell} r R_{n \ell}(r),
$$

onde $V_{e f}[\rho(r)]$ é o potencial efetivo de um elétron dado por:

$$
V_{e f}[\rho(r)]=-\frac{Z}{r}+V_{H}[\rho(r)]+\mu_{x c}[\rho(r)] .
$$

O primeiro termo da direita em (1.32) representa o potencial coulombiano sentido pelos elétrons devido ao núcleo, onde $Z$ é o número atômico; $\mu_{x c}$ é o potencial de troca-correlação na aproximação LDA e $V_{H}$ o potencial de Hartree; $\rho$ é a densidade de carga eletrônica dos orbitais ocupados:

$$
\rho(r)=\sum_{o c c}\left|R_{u \ell}(r)\right|^{2}
$$

Uma vez obtidos o conjunto de autovalores e funçōes de onda radiais do sistema com todos os elétrons, são selecionados os estados de valência. A partir destes o pseudopotencial é construído de forma a satisfazer quatro condições básicas (o número quântico principal $n$ será omitido para simplificar a notação): 
(I) As pseudofunções de onda, geradas a partir de um pseudopotencial, devem ser suaves e não possuir zeros.

(II) A pseudofunção de onda radial normalizada com momento angular $\ell\left(R_{\ell}^{p s}\right)$, deve ser igual ou tender rapidamente à função de onda radial normalizada com mesmo momento angular, obtida no cálculo de todos os elétrons $\left(R_{\ell}\right)$. Esta igualdade deve-se dar a partir de um certo raio de corte $r_{c \ell}$ :

$$
R_{\ell}^{p s}(r)=R_{\ell}(r), \quad r>r_{c \ell}
$$

(III) A carga contida dentro da região limitada pelo raio de corte $r_{c \ell}$, calculadas a partir das funções $R_{\ell}^{p s}$ e $R_{\ell}$ devem ser iguais:

$$
\int_{0}^{r_{c \ell}}\left|R_{\ell}^{p s}(r)\right|^{2} r^{2} d r=\int_{0}^{r_{c \ell}}\left|R_{\ell}(r)\right|^{2} r^{2} d r
$$

(IV) Os autovalores de valência obtidos no cálculo de todos os elétrons devem ser iguais aos obtidos com pseudopotencial:

$$
\varepsilon_{\ell}^{p s}=\varepsilon_{\ell}
$$

A pseudofunção de onda radial é construída de modo a satisfazer as três primeiras condições, tendo como referência a função de onda $R_{\ell}$. Uma vez que esta é construída, o pseudopotencial é calculado invertendo-se a equação (1.31). Como o pseudopotencial é obtido a partir de uma cálculo de muitos elétrons, este terá embutido os efeitos de blindagem dos elétrons de valência, portanto será um pseudopotencial blindado $(s c r)$ :

$$
V_{s c r . \ell}^{p s}(r)=\varepsilon_{\ell}-\frac{\ell(\ell+1)}{2 r^{2}}+\frac{1}{2 r R_{\ell}^{p s}(r)} \frac{d^{2}}{d r^{2}}\left[r R_{\ell}^{p s}(r)\right] \text {. }
$$

$\mathrm{Na}$ equação (1.37) podemos notar algumas caracteríticas do pseudopotencial que podem ser modeladas pela escolha da pseudofunção de onda. Por exemplo, para evitar singularidades no pseudopotencial a pseudofunção não deve possuir zeros (condição I). O pseudopotencial será uma função contínua e diferenciável se a pseudofunção possui derivada superior a segunda ordem. Pseudopotenciais profundos na região do caroço ou com singularidades na origem, podem ser evitadas se a pseudofunção se comportamenta como $r^{\ell}$ perto da origem.

Pseudopotenciais blindados são fortemente dependentes dos elétrons de valência do meio onde foram gerados. Esta dependência pode ser eliminada 
removendo as interações elétron-elétron de forma a obter um pseudopotencial iônico. Isto é, substraindo o potencial de Hartree e o potencial de trocacorrelação, calculados a partir das pseudofunções de onda do pseudopotencial blindado:

$$
V_{i o n, \ell}^{p s}(r)=V_{s c r, \ell}^{p s}-V_{H}\left[\rho^{p s}(r)\right]-\mu_{x c}\left[\rho^{p s}(r)\right]
$$

onde

$$
\rho^{p s}(r)=\sum_{v a l}\left|R_{\ell}^{p s}(r)\right|^{2},
$$

aqui a soma é somente nos elétrons de valência. Pseudopotenciais gerados da forma acima descrita são conhecidos como de norma conservada.

Comumente os pseudopotenciais iônicos são escritos como operadores contendo uma parte local (L) independente de $\ell$ e uma parte não-local na componente radial, dependente de $\ell$. Devido a esta não-localidade existir somente na componente angular e não na componente radial, chamaremos esta parte do operador de semi-local (SL), assim:

$$
V_{i o n, \ell}^{p s}(r)=V_{L}^{p s}(r)+V_{S L, \ell}^{p s}(r),
$$

onde

$$
V_{S L, \ell}^{p s}(r)=\sum_{m=-\ell}^{\ell} \Delta V_{\ell}^{p s}(r)|\ell m\rangle\langle\ell m|,
$$

com

$$
\Delta V_{\ell}(r)=V_{i o n, \ell}^{p s}(r)-V_{L}^{p s}(r),
$$

onde $|\ell m\rangle$ são os harmônicos esféricos normalizados e $\Delta V_{\ell}(r)$ é uma função espacialmente localizada, a qual é nula para $r>r_{c \ell}$, e delocalizada na componente angular.

Uma importante propriedade que devem satisfazer os pseudopotenciais está relacionada com a transferibilidade. Pseudopotenciais iônicos são construídos de forma a reproduzir as propriedades de espalhamento dos sistemas de referência obtidos a partir de cálculos considerando todos os elétrons. Um pseudopotencial é transferivel se consegue reproduzir estas propriedades em um ambiente distinto do qual foi gerado. Um critério de transferibilidade comumente usado para pseudopotenciais de norma conservada é dado por uma identidade da regra de soma de Friedel: ${ }^{23}$ 


$$
-\frac{1}{2}\left[r^{2} R^{2}(r, \varepsilon) \frac{\partial}{\partial \varepsilon} \frac{\partial}{\partial r} \ln [R(r, \varepsilon)]\right]_{\substack{\varepsilon \circledast \varepsilon_{c} \\ r=r_{c \ell}}}=\int_{0}^{r_{\iota \ell}} R^{2}\left(r, \varepsilon_{\ell}\right) r^{2} d r
$$

onde $R(r, \varepsilon)$ é a solução da equação de Schrödinger para uma energia $\varepsilon$ (não necessariamente um autovalor), a qual é regular em $r=0$. A derivada logarítmica radial na esquerda da equação (1.43) está relacionada com o deslocamento de fase do espalhamento. Se aplicamos esta identidade à pseudofunção radial $R_{\ell}^{p s}(r, \varepsilon)$ e a função de onda radial de todos os elétrons $R_{\ell}(r, \varepsilon)$, no ponto $r=r_{c \ell}$, vemos que o lado esquerdo das respectivas identidades serão iguais para pseudopotencial de norma conservada, i.e., que satisfazem a equação (1.35). Isto significa que o pseudopotencial e o potencial real terão a mesma variação, em primeira ordem na energia, que seus deslocamentos de fase de espalhamento quando transferidos para outros sistemas. Na prática, comparando as derivadas logarítmicas das duas funções de onda $R_{\ell}^{p s}$ e $R_{\ell}$, para um raio $r_{0} \geq r_{c \ell}$, considerando um intervalo de energia compreendido pela banda de valência e mínimo da banda de condução, podemos ter uma primeira aproximação da qualidade do pseudopotencial iônico.

\subsubsection{Pseudopotenciais Separáveis}

Uma considerável redução no número de integrais necessárias para calcular a parte semi-local do pseudopotencial iônico em um cálculo de estrutura de bandas foi introduzido por Kleinman e Bylander (1982) ${ }^{24}$ Neste método, a separação do pseudopotencial é feita não somente na componente do momento angular (semi-local) mas também na parte radial. Com isto o pseudopotencial é colocado em uma forma totalmente separável ou não-local (NL). Esta componente não-local é definida como:

$$
V_{N L, \ell}^{p s}(\mathbf{r})=\sum_{m=-\ell}^{\ell} \frac{\left|\Delta V_{\ell}(r) \Phi_{\ell m}^{p s}(\mathbf{r})\right\rangle\left\langle\Delta V_{\ell}(r) \Phi_{\ell m}^{p s}(\mathbf{r})\right|}{\left\langle\Phi_{\ell m}^{p s}(\mathbf{r})\left|\Delta V_{\ell}(r)\right| \Phi_{\ell m}^{p s}(\mathbf{r})\right\rangle}
$$

onde $\Delta V_{\ell}(r)$ é definido pela equação (1.42) e $\Phi_{\ell m}^{p s}(\mathbf{r})$ é a pseudofunção de onda de referência usada para calcular o pseudopotencial, definida por:

$$
\Phi_{\ell m}^{p s}(\mathbf{r})=R_{\ell}^{p s}(r) Y_{\ell m}(\theta, \varphi)
$$


A fórmula que conecta a forma não-local da semi-local é dada por:

$$
V_{N L, \ell}^{p s}(\mathbf{r})\left|\Phi_{\ell m}^{p s}(\mathbf{r})\right\rangle=\left|\Delta V_{\ell}(r) \Phi_{\ell m}^{p s}(\mathbf{r})\right\rangle
$$

No formalismo do espaço dos momento, a pseudofunção de onda monoeletrônica para o cristal $\Phi_{n, k}(\mathbf{r})$ é expandida em um conjunto de ondas planas, enquanto que os pseudopotenciais são representados pela sua transformada de Fourier. Os elementos de matriz da componente semi-local do pseudopotencial, equação (1.41), no espaço recíproco podem ser escritos como:

$$
V_{S L, \ell}^{p s}\left(\mathbf{q}, \mathbf{q}^{\prime}\right)=\frac{1}{\Omega_{a}}\left[\int_{0}^{\infty} \Delta V_{\ell}(r) j_{\ell}(q r) j_{\ell}\left(q^{\prime} r\right) r^{2} d r\right] \sum_{m=-\ell}^{\ell} Y_{\ell m}(\hat{\mathbf{q}}) Y_{\ell m}^{*}\left(\hat{\mathbf{q}}^{\prime}\right)
$$

onde $q^{\prime}=\left|\mathbf{k}+\mathbf{G}^{\prime}\right|$ é o vetor de onda, $\Omega_{a}$ é o volume por átomo na célula primitiva e $j_{\ell}(q r)$ são as funções de Bessel esféricas. Seja $n_{q}$ o número de vetores de onda da expansão por átomo e $n_{a}$ o número de átomos do sistema. Da equação (1.47) vemos que o número de operaçōes necessárias para calcular o termo semi-local no espaço recíproco para um dado $\ell$ fixo, é da ordem de $O\left(n_{a} \times n_{q}^{2}\right)$. Considerando agora o termo não-local do pseudopotencial no método de Kleinman-Bylander, equação (1.44), os elementos de matriz no espaço recíproco são dados por:

$$
\begin{aligned}
V_{N L, \ell}^{p s}\left(\mathbf{q}, \mathbf{q}^{\prime}\right) & =\frac{(4 \pi)^{2}}{\Omega_{a}\left\langle\Phi_{\ell m}^{p s}(\mathbf{r})\left|\Delta V_{\ell}(r)\right| \Phi_{\ell m}^{p s}(\mathbf{r})\right\rangle}\left[\int_{0}^{\infty} R_{\ell}^{p s}(r) \Delta V_{\ell}(r) j_{\ell}(q r) r^{2} d r\right] \\
& \times\left[\int_{0}^{\infty} R_{\ell}^{p s}(r) \Delta V_{\ell}(r) j_{\ell}\left(q^{\prime} r\right) r^{2} d r\right] \sum_{m=-\ell}^{\ell} Y_{\ell m}(\hat{\mathbf{q}}) Y_{\ell m}^{*}\left(\hat{\mathbf{q}}^{\prime}\right) .
\end{aligned}
$$

A fatorização em um produto de integrais envolvendo um único valor de $q$, permite que o número de operações necessárias para calcular o termo nãolocal da equação (1.48) seja agora da ordem de $O\left(n_{a} \times n_{q}\right)$ por integral. Isto é, a dependência quadrática no número de vetores de onda é reduzida a uma dependência linear. Este procedimento é numericamente mais eficiente devido a que agora não é necessário armazenar a matriz não-local completa, mas somente elementos de vetor de dimensão $n_{q}$, permitindo também que os cálculos sejam mais rápidos. 
Pseudopotenciais iônicos transformados na forma totalmente separável de Kleinman-Bylander tem apresentado problemas quando utilizados, por exemplo, em cálculos da energia de ligação e estrutura de bandas do $G a A s .{ }^{25}$ Estes problemas estão relacionados com a aparição de estados ligados espúrios em alguns átomos, chamados de "estados fantasmas". Bylander e Kleinman ${ }^{26}$ propuseram regras empíricas para evitar a aparição destes estados e Gonze et $a l^{25}$ formularam um teorema de existência a partir de uma análise detalhada do método de separação. A identificação dos "estados fantasmas" é feita a partir de eventuais singularidades que se apresentam na derivada logarítmica do pseudopotencial não local $V_{N L, \ell}^{p s}$, para um determinado orbital $\ell$, que não possui equivalente no cálculo com todos os elétrons. Na prática estes podem ser evitados variando-se levemente o raio de corte $r_{c \ell}$ para o pseudopotencial nãolocal onde os "estados fantasmas" são detectados, porém este procedimento compromete a sua transferibilidade. Um outro procedimento comumente utilizado é construir o pseudopotencial local a partir do pseudopotencial iônico que na transformação de Kleinman-Bylander apresenta problemas de "estados fantasmas".

\subsubsection{Pseudopotencial de Troullier-Martins}

Devido ao sucesso dos pseudopotenciais ab initio no cálculo da estrutura eletrônica de sólidos, moléculas e superfícies, grande tem sido a procura por novos pseudopotenciais que permitam cálculos mais complicados e que sejam computacionalmente eficientes. Pseudopotenciais suaves são aqueles que tem uma rápida convergência em cálculos de energia total quando o número de ondas planas no conjunto base é aumentado. Troullier e Martins (1990), ${ }^{27}$ baseados no método de Kerker, ${ }^{28}$ desenvolveram um pseudopotencial de norma conservada que tem posibilitado cálculos de energia total envolvendo elementos da primeira fila da Tabela Periódica ${ }^{29}$ e metais de transição. ${ }^{30}$ Pelo fato dos elementos da primeira fila não possuírem elétrons de caroço, seus pseudopotenciais do orbital $p(\ell=1)$ são muito profundos, precisando de um grande número de ondas planas no conjunto base para sua correta descrição. O mesmo acontece com os pseudopotenciais do orbital $d(\ell=2)$ dos metais de transição. Originalmente no método de Kerker as pseudofunçōes de onda radial são definidas como: 


$$
R_{\ell}^{p s}(r)= \begin{cases}R_{\ell}(r), & r \geq r_{c \ell} \\ r^{\ell} \exp [p(r)], & r \leq r_{c \ell}\end{cases}
$$

onde $R_{\ell}(r)$ é a pseudofunção de onda radial de todos os elétrons e $p(r)$ é um polinômio de ordem quatro. A determinação dos coeficientes de $p(r)$ é obtida impondo-se a condição de conservação da norma e a continuidade de $R_{\ell}^{p s}(r)$ e de suas duas primeiras derivadas. O coeficiente $c_{1}$ foi excluído para evitar singularidades do pseudopotencial blindado $V_{s c r, \ell}^{p s}(r)$ em $r=0$. Troullier e Martins introduziram um novo polinômio, de ordem seis em $r^{2}$,

$$
p(r)=c_{0}+c_{2} r^{2}+c_{4} r^{4}+c_{6} r^{6}+c_{8} r^{8}+c_{10} r^{10}+c_{12} r^{12}
$$

onde agora os sete coeficientes de $p(r)$ são determinados impondo-se as sete seguintes condições:

(I) Conservação da norma para a carga dentro do raio $r \leq r_{\ell}$ :

$$
2 c_{0}+\ln \left[\int_{0}^{r_{c \ell}} r^{2(\ell+1)} \exp \left[2 p(r)-2 c_{0}\right] d r\right]=\ln \left[\int_{0}^{r_{c \ell}}\left|R_{\ell}(r)\right|^{2} r^{2} d r\right]
$$

(II)-(VI) Continuidade da pseudofunção de onda e de suas quatro primeiras derivadas em $r=r_{c \ell}$. Estas condições impõem também a continuidade de $V_{s c r \ell \ell}^{p s}(r)$ e de suas duas primeiras derivadas, no mesmo ponto $r=r_{c \ell}$ :

$$
\begin{aligned}
p\left(r_{c \ell}\right)= & \ln \left[\frac{P\left(r_{c \ell}\right)}{r_{c \ell}^{\ell+1}}\right], \\
p^{\prime}\left(r_{c \ell}\right)= & \frac{P^{\prime}\left(r_{c \ell}\right)}{P\left(r_{c \ell}\right)}-\frac{\ell+1}{r_{c \ell}}, \\
p^{\prime \prime}\left(r_{c \ell}\right)= & 2 V_{s c r \cdot \ell}\left(r_{c \ell}\right)-2 \varepsilon_{\ell}-\frac{2(\ell+1)}{r_{c \ell}} p^{\prime}\left(r_{c \ell}\right)-\left[p^{\prime}\left(r_{c \ell}\right)\right]^{2}, \\
p^{\prime \prime \prime}\left(r_{c \ell}\right)= & 2 V_{s c r \ell \ell}^{\prime}\left(r_{c \ell}\right)+\frac{2(\ell+1)}{r_{c \ell}^{2}} p^{\prime}\left(r_{c \ell}\right)-\frac{2(\ell+1)}{r_{c \ell}} p^{\prime \prime}\left(r_{c \ell}\right) \\
& -2 p^{\prime}\left(r_{c \ell}\right) p^{\prime \prime}\left(r_{c \ell}\right),
\end{aligned}
$$




$$
\begin{aligned}
p^{\prime \prime \prime \prime}\left(r_{c \ell}\right)= & 2 V^{\prime \prime}\left(r_{c \ell}\right)-\frac{4(\ell+1)}{r_{c \ell}^{3}} p^{\prime}\left(r_{c \ell}\right)+\frac{4(\ell+1)}{r_{c \ell}^{2}} p^{\prime \prime}\left(r_{c \ell}\right) \\
& -\frac{2(\ell+1)}{r_{c \ell}} p^{\prime \prime \prime}\left(r_{c \ell}\right)-2\left[p^{\prime \prime}\left(r_{c \ell}\right)\right]^{2}-2 p^{\prime}\left(r_{c \ell}\right) p^{\prime \prime \prime}\left(r_{c \ell}\right),
\end{aligned}
$$

onde $P(r)=r R_{\ell}(r)$ e $V_{s c r \ell}(r)$ é o potencial atômico blindado de todos os elétrons.

(VII) A curvatura zero na origem para o pseudopotencial blindado que determina a condição de suavidade, $V_{s c r, \ell}^{\prime \prime}(r=0)=0$, é imposta pela equação:

$$
c_{2}^{2}+c_{4}(2 \ell+5)=0 .
$$

Uma vez avaliados os coeficientes do polinômio $p(r)$, as pseudofunções de onda radiais são obtidas a partir da equação (1.49). O pseudopotencial blindado $V_{s c r \ell}^{p s}(r)$ é obtido invertendo-se a equação de Schrödinger (1.37), e pode ser escrito explicitamente como:

$$
V_{s c r, \ell}^{p s}(r)= \begin{cases}V_{s c r, \ell}(r), & r \geq r_{c \ell} \\ \varepsilon_{\ell}+\frac{\ell+1}{r} \frac{p^{\prime}(r)}{2}+\frac{\left.p^{\prime \prime}(r)+\mid p^{\prime}(r)\right]^{2}}{2}, & r \leq r_{c \ell} .\end{cases}
$$

$\mathrm{Na}$ equação (1.58) pode ser visto que o pseudopotencial gerado no esquema de Troullier-Martins é uma função analítica dentro do raio de corte $r_{c \ell}$. A condição (VII) assegura um bom comportamento na origem, i.e., sem singularidades.

\subsection{Energia Total no Espaço dos Momento}

Nas seções precedentes foram discutidos os formalismos que permitem a obtenção da energia total para o estado fundamental de um sistema de elétrons em presença de um potencial externo. O cálculo da energia total para um cristal, que é definida sem considerar a energia devido aos caroços isolados, vêse grandemente simplificada considerando-se a periodicidade da rede. A simetria do cristal permite que os cálculos sejam restritos a uma célula unitária que é repetida no espaço. Isto permite que as expressões mais relevantes da energia total do cristal (energia cinética e potenciais) sejam representadas no espaço 
dos momentos por meio de uma transformada de Fourier as quais podem ser expressadas como somas sobre os vetores da rede recíproca $\mathbf{G}$ ao invés de integrais sobre os vetores de onda $\mathrm{k}$. Neste formalismo, primeiramente introduzido por Ihm, Zunger e Cohen (1979), ${ }^{31}$ as pseudofunções de onda mono-eletrônicas são substituídas por pseudofunções de onda de Bloch, $\Phi_{n, \mathrm{k}}$, as quais são expandidas em um conjunto de ondas planas. Assim para um elétron com vetor de onda $\mathrm{k}$ na banda $n$ temos que:

$$
\Phi_{n, \mathbf{k}}(\mathbf{r})=\frac{1}{\sqrt{N_{c} v}} \sum_{\mathrm{G}} C_{n}(\mathbf{k}+\mathbf{G}) \exp [i(\mathbf{k}+\mathbf{G}) \cdot \mathbf{r}],
$$

onde $v$ é o volume da célula unitária e $N_{c}$ o número de células unitárias contidas no cristal. $\mathbf{G}$ é um vetor da rede recíproca e $C_{n}(\mathbf{k}+\mathbf{G})$ são os coeficientes de expansão que são interpretados como componentes de Fourier da pseudofunção de onda. A energia total do cristal pode ser expressada como:

$$
E_{\text {total }}=E_{\text {cin }}^{0}+E_{\text {el,ion }}+E_{\text {ion }, \text { ion }},
$$

onde $E_{\text {cin }}^{0}$ é a energia cinética dos elétrons não interagentes, $E_{i o n, i o n}$ é a energia de interação entre os íons da rede e $E_{e l, i o n}$ é a energia de interação eletrônica na presença de um potencial externo devido aos íons. A interação íon-íon pode ser substituída rigorosamente por uma interação entre íons pontuais esfericamente simétricos e afastados o suficiente de modo a não ter superposição entre eles. Este termo pode ser escrito como:

$$
E_{\text {ion, ion }}=\sum_{\mu} \sum_{\nu} \frac{Z_{\mu} Z_{\nu}}{\left|\mathbf{R}_{\mu}-\mathbf{R}_{\nu}\right|},
$$

onde $(\mu, \nu)$ representam íons e $\left(Z_{\mu}, Z_{\nu}\right)$ suas respectivas cargas de valência. $\mathbf{R}_{\mu}$ e $\mathbf{R}_{\nu}$ são vetores da rede que representam as posições atômicas. O termo de interação eletrônica da equação (1.60) representa a energia do estado fundamental dos elétrons de valência, dentro da aproximação LDA. Este termo pode ser determinado a partir das equações de Kohn-Sham para o cristal, as que podem ser escritas como:

$$
\left[-\frac{1}{2} \nabla^{2}+V_{e f}(\mathbf{r})-E_{n}(\mathbf{k})\right] \Phi_{n, \mathbf{k}}(\mathbf{r})=0,
$$

com

$$
V_{e f}(\mathbf{r})=\sum_{\mu, \ell} V_{i o n, \ell}^{p s}\left(\mathbf{r}-\mathbf{R}_{\mu}\right)+V_{H}(\mathbf{r})+V_{x c}(\mathbf{r}),
$$


onde o primeiro termo da direita em (1.63) representa o pseudopotencial iônico no cristal que é uma contribuição devido a todos os íons do sistema. A densidade de carga é dada por:

$$
\rho(\mathbf{r})=\frac{v}{(2 \pi)^{3}} \int_{Z B} d \mathbf{k} \rho_{\mathbf{k}}(\mathbf{r}),
$$

com

$$
\rho_{\mathrm{k}}(\mathrm{r})=\sum_{n}^{o c c}\left|\Phi_{n, \mathrm{k}}(\mathrm{r})\right|^{2} .
$$

A transformada de Fourier do potencial efetivo $V_{e f}(\mathbf{r})$ é dada por:

$$
V_{e f}\left(\mathbf{k}+\mathbf{G}, \mathbf{k}+\mathbf{G}^{\prime}\right) \equiv \frac{1}{N_{c} v} \int d \mathbf{r} \exp [-i(\mathbf{k}+\mathbf{G}) \cdot \mathbf{r}] V_{e f}(\mathbf{r}) \exp \left[-i\left(\mathbf{k}+\mathbf{G}^{\prime}\right) \cdot \mathbf{r}\right] \text {. }
$$

Devido a $V_{x c}(\mathbf{r})$ na aproximação LDA e $V_{H}(\mathbf{r})$ serem potenciais locais, suas componentes de Fourier dependem exclusivamente de $\mathbf{G}-\mathbf{G}^{\prime}$, logo o potencial efetivo no espaço recíproco é escrito como:

$$
V_{e f}\left(\mathrm{k}+\mathbf{G}, \mathbf{k}+\mathbf{G}^{\prime}\right)=V_{i o n}^{p s}\left(\mathbf{k}+\mathbf{G}, \mathbf{k}+\mathbf{G}^{\prime}\right)+V_{H}\left(\mathbf{G}-\mathbf{G}^{\prime}\right)+V_{x c}\left(\mathbf{G}-\mathbf{G}^{\prime}\right) .
$$

O primeiro termo da direita de (1.67) é a componente de Fourier do pseudopotencial iônico total, que será especificado posteriormente. Para obter a equação de Kohn-Sham no espaço dos momentos, multiplicamos $\Phi_{n, \mathrm{k}}^{*}(\mathrm{r})$ pela esquerda da equação (1.62) e integramos em todo o espaço:

$$
\int d \mathbf{r} \Phi_{n, \mathbf{k}}^{*}(\mathbf{r})\left[-\frac{1}{2} \nabla^{2}+V_{e f}(\mathbf{r})+E_{n}(\mathbf{k})\right] \Phi_{n, \mathbf{k}}(\mathbf{r})=0 .
$$

Separando o termo do potencial efetivo:

$$
\begin{aligned}
\int d \mathbf{r} & \Phi_{n, \mathbf{k}}^{*}(\mathbf{r}) V_{e f}(\mathbf{r}) \Phi_{n, \mathbf{k}}(\mathbf{r}) \\
= & \frac{1}{N_{c} v} \sum_{\mathrm{G}, \mathrm{G}^{\prime}} C_{n}^{*}(\mathrm{k}+\mathbf{G}) C_{n}\left(\mathbf{k}+\mathbf{G}^{\prime}\right) \\
& \times \int d \mathbf{r} \exp [-i(\mathbf{k}+\mathbf{G}) \cdot \mathbf{r}] V_{e f}(\mathrm{r}) \exp \left[-i\left(\mathbf{k}+\mathbf{G}^{\prime}\right) \cdot \mathbf{r}\right] \\
& =\sum_{\mathrm{G}, \mathrm{G}^{\prime}} C_{n}^{*}(\mathrm{k}+\mathbf{G}) C_{n}\left(\mathrm{k}+\mathbf{G}^{\prime}\right) V_{e f}\left(\mathrm{k}+\mathbf{G}, \mathbf{k}+\mathbf{G}^{\prime}\right) .
\end{aligned}
$$


O termo restante fica como:

$$
\begin{aligned}
\int d \mathbf{r} & \Phi_{n, \mathrm{k}}^{*}(\mathbf{r})\left[-\frac{1}{2} \nabla_{\mathrm{r}}^{2}++E_{n}(\mathbf{k})\right] \Phi_{n, \mathbf{k}}(\mathbf{r}) \\
= & \frac{1}{N_{c} v} \sum_{\mathrm{G}^{\prime} \mathrm{G}^{\prime}} C_{n}^{*}(\mathbf{k}+\mathbf{G}) C_{n}\left(\mathbf{k}+\mathbf{G}^{\prime}\right) \\
& \times \int d \mathbf{r}\left[\frac{\left(\mathbf{k}+\mathbf{G}^{\prime}\right)^{2}}{2}+E_{n}(\mathbf{k})\right] \exp \left[-i\left(\mathbf{G}-\mathbf{G}^{\prime}\right) \cdot \mathbf{r}\right] \\
= & \sum_{\mathrm{G}, \mathrm{G}^{\prime}} C_{n}^{*}(\mathbf{k}+\mathbf{G}) C_{n}\left(\mathbf{k}+\mathbf{G}^{\prime}\right)\left[\frac{\left(\mathbf{k}+\mathbf{G}^{\prime}\right)^{2}}{2}+E_{n}(\mathbf{k})\right] \delta_{\mathrm{G}^{\prime}, G^{\prime}}
\end{aligned}
$$

onde utilizamos a identidade:

$$
\frac{1}{N_{c} v} \int d \mathbf{r} \exp \left[i\left(\mathbf{G}-\mathbf{G}^{\prime}\right) \cdot \mathbf{r}\right]=\delta_{\mathrm{G} \mathrm{G}^{\prime}}
$$

Substituindo (1.69) e (1.70) em (1.68) temos que:

$$
\sum_{\mathrm{G}^{\prime}}\left[\frac{\left(\mathrm{k}+\mathbf{G}^{\prime}\right)^{2}}{2} \delta_{\mathrm{G}, \mathrm{G}^{\prime}}+V_{e f}\left(\mathbf{k}+\mathbf{G}, \mathbf{k}+\mathbf{G}^{\prime}\right)\right] C_{n}\left(\mathbf{k}+\mathbf{G}^{\prime}\right)=E_{n}(\mathbf{k}) C_{n}(\mathbf{k}+\mathbf{G})
$$

A análise de Fourier das equações de Kohn-Sham, que são equações diferenciais de segunda ordem em $\Phi_{n, k}(r)$, permite que estas sejam transformadas em um conjunto de equações lineares nas componentes de Fourier $C_{n}(\mathbf{k}+\mathbf{G})$. $\mathrm{A}$ energia pseudopotencial pode ser escrita como:

$$
\begin{aligned}
E_{p s} & =\sum_{\mu, \ell} \int d \mathbf{r} \Phi_{n, \mathrm{k}}^{*}(\mathbf{r}) V_{i o n, \ell}^{p s}\left(\mathbf{r}-\mathbf{R}_{\mu}\right) \Phi_{n, \mathrm{k}}(\mathbf{r}) \\
& =\frac{1}{N_{c} v} \sum_{\mu, \ell} \sum_{\mathrm{G}, \mathrm{G}^{\prime}} C_{n}^{*}(\mathbf{k}+\mathbf{G}) C_{n}\left(\mathbf{k}+\mathbf{G}^{\prime}\right) \\
& \times \int d \mathbf{r} \exp [-i(\mathbf{k}+\mathbf{G}) \cdot \mathbf{r}] V_{i o n, \ell}^{p s}\left(\mathbf{r}-\mathbf{R}_{\mu}\right) \exp \left[-i\left(\mathbf{k}+\mathbf{G}^{\prime}\right) \cdot \mathbf{r}\right]
\end{aligned}
$$

Fazendo a mudança de variável $\mathbf{r} \rightarrow \mathbf{r}-\mathbf{R}_{\mu}$, obtemos: 


$$
\begin{aligned}
E_{p s} & =\frac{1}{N_{c} v} \sum_{\mu, \ell} \sum_{\mathrm{G}, \mathrm{G}^{\prime}} C_{n}^{*}(\mathbf{k}+\mathbf{G}) C_{n}\left(\mathbf{k}+\mathbf{G}^{\prime}\right) \exp \left[i\left(\mathbf{G}-\mathbf{G}^{\prime}\right) \cdot \mathbf{R}_{\mu}\right] \\
& \times \int d \mathbf{r} \exp [-i(\mathbf{k}+\mathbf{G}) \cdot \mathbf{r}] V_{i o n}^{p s}, \ell(\mathbf{r}) \exp \left[i\left(\mathbf{k}+\mathbf{G}^{\prime}\right) \cdot \mathbf{r}\right] \\
& =\sum_{\mathrm{G}, \mathrm{G}^{\prime}} C_{n}^{*}(\mathbf{k}+\mathbf{G}) C_{n}\left(\mathbf{k}+\mathbf{G}^{\prime}\right) S\left(\mathbf{G}-\mathbf{G}^{\prime}\right) V_{i o n}^{p s}\left(\mathbf{k}+\mathbf{G}, \mathbf{k}+\mathbf{G}^{\prime}\right)
\end{aligned}
$$

$S\left(\mathbf{G}^{\prime}-\mathbf{G}\right)$ é definido como o fator de estrutura:

$$
S\left(\mathbf{G}-\mathbf{G}^{\prime}\right) \equiv \frac{1}{N} \sum_{\mu} \exp \left[i\left(\mathbf{G}-\mathbf{G}^{\prime}\right) \cdot \mathbf{R}_{\mu}\right]
$$

onde $N$ é o número de átomos na célula primitiva de volume $v$. Na equação (1.74), $V_{i o n}^{p s}\left(\mathrm{k}+\mathrm{G}, \mathrm{k}+\mathbf{G}^{\prime}\right)$ é o fator de forma iônico, definido por:

$$
V_{i o n}^{p s}\left(\mathbf{q}, \mathbf{q}^{\prime}\right) \equiv \frac{1}{\Omega_{a}} \sum_{\ell} \int d \mathbf{r} \exp [-i \mathbf{q} \cdot \mathbf{r}] V_{i o n, \ell}^{p s}(\mathbf{r}) \exp \left[-i \mathbf{q}^{\prime} \cdot \mathbf{r}\right]
$$

onde $\Omega_{a}$ é o volume por átomo e $\mathbf{q}^{\prime}=\mathbf{k}+\mathbf{G}^{\prime}$. Devido a separação do pseudopotencial iônico em uma componente local e outra não local, podemos calcular suas respectivas componentes no espaço recíproco considerando:

$$
\sum_{\ell} V_{i o n, \ell}^{p s}(\mathbf{r})=V_{L}^{p s}(|\mathbf{r}|)+\sum_{\ell} V_{N L, \ell}^{p s}(\mathbf{r})
$$

logo a equação (1.76) também pode ser separada em componentes local e nãolocal:

$$
V_{i o n}^{p s}\left(\mathbf{q}, \mathbf{q}^{\prime}\right)=V_{L}^{p s}\left(\mathbf{q}, \mathbf{q}^{\prime}\right)+\sum_{\ell} V_{N L, \ell}^{p s s}\left(\mathbf{q}, \mathbf{q}^{\prime}\right)
$$

Como a parte local do pseudopotencial depende unicamente de $r=|\mathbf{r}|$, sua transformada de Fourier será:

$$
V_{L}^{p s}\left(\mathrm{q}, \mathrm{q}^{\prime}\right)=\frac{1}{\Omega_{a}} \int d \mathbf{r} \exp \left[-i\left(\mathbf{G}-\mathbf{G}^{\prime}\right) \cdot \mathbf{r}\right] V_{L}^{p s}(|\mathbf{r}|)=V_{L}^{p s}\left(\mathbf{G}-\mathbf{G}^{\prime}\right)
$$

Para o termo não-local temos que: 


$$
V_{N L, \ell}^{p s}\left(\mathbf{q}, \mathbf{q}^{\prime}\right)=\frac{1}{\Omega_{a}} \int d \mathbf{r} \exp [-i \mathbf{q} \cdot \mathbf{r}] V_{N L, \ell}^{p s}(\mathbf{r}) \exp \left[i \mathbf{q}^{\prime} \cdot \mathbf{r}\right]
$$

Usando a identidade:

$$
\exp [i \mathbf{q} \cdot \mathbf{r}]=4 \pi \sum_{\ell=0}^{\infty} \sum_{m=-\ell}^{\ell} i^{\ell} j_{\ell}(q r) Y_{\ell m}(\hat{\mathbf{r}}) Y_{\ell m}(\hat{\mathbf{q}}),
$$

onde $j_{\ell}$ são as funções esféricas de Bessel e $Y_{\ell m}$ os harmônicos esféricos. Substituindo (1.81) em (1.80) temos que:

$$
V_{N L, \ell}^{p s}\left(\mathbf{q}, \mathbf{q}^{\prime}\right)=\frac{(4 \pi)^{2}}{\Omega_{a}}\left[\int_{0}^{\infty} j_{\ell}(q r) j_{\ell}\left(q^{\prime} r\right) V_{N L, \ell}^{p s}(\mathbf{r}) r^{2} d r\right] \sum_{m=-\ell}^{\ell} Y_{\ell m}(\hat{\mathbf{q}}) Y_{\ell m}\left(\hat{\mathbf{q}}^{\prime}\right)
$$

Utilizando o teorema de adição dos armônicos esféricos:

$$
\sum_{m=-\ell}^{\ell} Y_{\ell m}(\hat{\mathbf{q}}) Y_{\ell m}\left(\hat{\mathbf{q}}^{\prime}\right)=\frac{2 \ell+1}{4 \pi} P_{\ell}\left(\hat{\mathbf{q}} \cdot \hat{\mathbf{q}}^{\prime}\right)
$$

onde $P_{\ell}$ são os polinômios de Legendre. Logo a componente de Fourier do termo não-local do pseudopotencial iônico pode ser expressada por:

$$
V_{N L, \ell}^{p s}\left(\mathbf{q}, \mathbf{q}^{\prime}\right)=\frac{4 \pi}{\Omega_{a}}(2 \ell+1) P_{\ell}\left(\hat{\mathbf{q}} \cdot \hat{\mathbf{q}}^{\prime}\right)\left[\int_{0}^{\infty} j_{\ell}(q r) j_{\ell}\left(q^{\prime} r\right) V_{N L, \ell}^{p s}(\mathbf{r}) r^{2} d r\right] .
$$

Utilizando a forma separável de Kleinman-Bylander ${ }^{24}$ da equação (1.44) para o pseudopotencial não-local $V_{N L, \ell}^{p s}(\mathrm{r})$, obtemos finalmente:

$$
\begin{aligned}
& V_{N L, \ell}^{p s}\left(\mathbf{q}, \mathbf{q}^{\prime}\right)=\frac{4 \pi}{\Omega_{a}} \frac{(2 \ell+1) P_{\ell}\left(\hat{\mathbf{q}} \cdot \hat{\mathbf{q}}^{\prime}\right)}{\left\langle\Phi_{\ell m}^{p s}(\mathbf{r})\left|\Delta V_{\ell}(r)\right| \Phi_{\ell m}^{p s}(\mathbf{r})\right\rangle} \\
& \quad \times \int_{0}^{\infty} j_{\ell}(q r) \Delta V_{\ell}(r) R_{\ell}^{p s}(r) r^{2} d r \int_{0}^{\infty} j_{\ell}\left(q^{\prime} r\right) \Delta V_{\ell}(r) R_{\ell}^{p s}(r) r^{2} d r
\end{aligned}
$$

onde 


$$
\left\langle\Phi_{\ell m}^{p s}(\mathbf{r})\left|\Delta V_{\ell}(r)\right| \Phi_{\ell m}^{p s}(\mathbf{r})\right\rangle=\int_{0}^{\infty}\left[R_{\ell}^{p s}(r) \Delta V_{\ell}(r)\right]^{2} r^{2} d r .
$$

Substituindo (1.78) e (1.74) obtemos a seguinte expressão para a energia pseudopotencial:

$$
\begin{aligned}
E_{p s} & =\sum_{\mathrm{G}, \mathrm{G}^{\prime}} C_{n}^{*}(\mathbf{k}+\mathbf{G}) C_{n}\left(\mathbf{k}+\mathbf{G}^{\prime}\right) S\left(\mathbf{G}-\mathbf{G}^{\prime}\right) \\
& \times\left[V_{L}^{p s}\left(\mathbf{G}-\mathbf{G}^{\prime}\right)+\sum_{\ell} V_{N L, \ell}^{p s}\left(\mathbf{k}+\mathbf{G}, \mathbf{k}+\mathbf{G}^{\prime}\right)\right] .
\end{aligned}
$$

A densidade de carga para um dado vetor de onda pode ser escrita como:

$$
\rho_{\mathrm{k}}(\mathbf{r})=\sum_{n}^{o c c} \rho_{n, \mathbf{k}}(\mathbf{r})
$$

onde $\rho_{n, \mathbf{k}}(\mathbf{r})=\left|\Phi_{n, \mathbf{k}}(\mathbf{r})\right|^{2}$. A transformada de Fourier de $\rho_{n, \mathbf{k}}(\mathbf{r})$ é dada por:

$$
\rho_{n, \mathbf{k}}(\mathbf{G})=\frac{1}{N_{c} v} \sum_{\mathbf{G}^{\prime}} C_{n}^{*}\left(\mathbf{k}+\mathbf{G}^{\prime}+\mathbf{G}\right) C_{n}\left(\mathbf{k}+\mathbf{G}^{\prime}\right) .
$$

Logo a energia pseudopotencial em função da densidade de carga no espaço recíproco pode ser expressada como:

$$
\begin{aligned}
E_{p s} & =N_{c} v \sum_{\mathbf{G}} \rho(\mathbf{G}) S(\mathbf{G}) V_{L}^{p s}(\mathbf{G}) \\
& +\sum_{n, \ell, \mathbf{G}, \mathbf{G}^{\prime}} C_{n}^{*}(\mathbf{k}+\mathbf{G}) C_{n}\left(\mathbf{k}+\mathbf{G}^{\prime}\right) S\left(\mathbf{G}-\mathbf{G}^{\prime}\right) V_{N L, \ell}^{p s}\left(\mathbf{k}+\mathbf{G}, \mathbf{k}+\mathbf{G}^{\prime}\right) .
\end{aligned}
$$

De forma análoga, a energia total do sistema é escrita como:

$$
E_{\text {total }}=E_{\text {cin }}^{0}+E_{\text {el,ion }}+E_{\text {ion }, \text { ion }},
$$

com

$$
E_{\text {cin }}^{0}=\sum_{n, \mathrm{G}}\left|C_{n}(\mathrm{k}+\mathrm{G})\right|^{2}(\mathrm{k}+\mathrm{G})^{2}
$$




$$
\begin{aligned}
& E_{\text {el,ion }}=N_{c} v \sum_{\mathrm{G}} \rho(\mathbf{G})\left[S(\mathbf{G}) V_{L}^{p s}(\mathbf{G})+V_{H}(\mathbf{G})+\epsilon_{x c}(\mathbf{G})\right] \\
& \quad+\sum_{n, \ell, \mathrm{G}, \mathrm{G}^{\prime}} C_{n}^{*}(\mathbf{k}+\mathbf{G}) C_{n}\left(\mathbf{k}+\mathbf{G}^{\prime}\right) S\left(\mathbf{G}-\mathbf{G}^{\prime}\right) V_{N L, \ell}^{p s}\left(\mathbf{k}+\mathbf{G}, \mathbf{k}+\mathbf{G}^{\prime}\right) \\
& E_{\text {ion,ion }}=\sum_{\mu} \sum_{\nu} \frac{Z_{\mu} Z_{\nu}}{\left|\mathbf{R}_{\mu}-\mathbf{R}_{\nu}\right|} .
\end{aligned}
$$

O potencial de Hartree para uma densidade de carga $\rho(\mathbf{r})$, é obtido a partir da solução da equação de Poisson:

$$
\nabla^{2} V_{H}(\mathbf{r})=-4 \pi \rho(\mathbf{r})
$$

O fator de forma correspondente é dado por:

$$
V_{H}(\mathbf{G})=\frac{-4 \pi \rho(\mathbf{G})}{|\mathbf{G}|^{2}} \text {. }
$$

No caso do potencial de troca-correlação, este é obtido na aproximação LDA por meio da equação (1.17). O cálculo da energia total, requer na prática de um tratamento especial nos termos divergentes. Estes termos são: a energia pseudopotencial em $\mathbf{G}-\mathbf{G}^{\prime}=0$, a energia de Hartree em $\mathbf{G}=0$ e a energia eletrostática entre os íons em $\mathbf{R}=0$. Estas três energias podem ser manipuladas convenientemente de forma a evitar as singularidades. É extremamente difícil calcular a energia $E_{\text {ion.ion }}$ usando somas de termos coulombianos tanto no espaço real como no recíproco, devido a que esta interação é de longo alcance. Ewald (1921) ${ }^{32}$ desenvolveu um método de rápida convergência para determinar somas de termos coulombianos em redes periódicas. Este método é baseado na seguinte identidade:

$$
\begin{aligned}
\sum_{\mathrm{L}} \frac{1}{\left|\mathbf{R}_{1}+\mathbf{L}+\mathbf{R}_{2}\right|}= & \frac{2 \pi}{\Omega} \sum_{\mathbf{G}} \int_{0}^{\zeta} \frac{1}{\rho^{3}} \exp \left[-\frac{|\mathbf{G}|^{2}}{4 \rho^{2}}\right] \exp \left[i\left(\mathbf{R}_{1}-\mathbf{R}_{2}\right) \cdot \mathbf{G}\right] d \rho \\
& +\frac{2}{\sqrt{\pi}} \sum_{\mathrm{L}} \int_{\zeta}^{\infty} \exp \left[-\left|\mathbf{R}_{1}+\mathbf{L}+\mathbf{R}_{2}\right|^{2} \rho^{2}\right] d \rho,
\end{aligned}
$$

onde $\mathrm{L}$ são vetores da rede e $\left(\mathbf{R}_{1}, \mathbf{R}_{2}\right)$, posições atômicas. Esta identidade é válida para todo valor positivo de $\zeta$. A energia total é calculada 
excluíndo-se os termos divergentes, introduzindo-se outros termos compensatórios e escrevendo-se a soma dos três termos divergentes como:

$$
E_{p s}+E_{H}+E_{\text {ion }, i o n}=\tilde{E}_{p s}+\tilde{E}_{H}+\gamma_{E w a l d}+Z \alpha
$$

onde o til indica que os termos divergentes em $E_{H}$ e $E_{p s}$ foram excluídos. $\gamma_{E \text { wald }}$ representa a energia eletrostática dos íons pontuais em um gás uniforme de elétrons de valência, excluíndo o termo divergente. $\alpha$ é a correção do potencial eletrostático real devido a introdução do pseudopotencial. Estes dois termos são representados por:

$$
\begin{aligned}
\gamma_{\text {Ewald }} & =\sum_{\mu \neq \nu} \frac{Z_{\mu} Z_{\nu}}{\left|\mathbf{R}_{\mu}-\mathbf{R}_{\nu}\right|}-\frac{1}{2} \frac{1}{\Omega_{a}} \int d \mathbf{r} \frac{Z^{2}}{|\mathbf{r}|} \\
& =\sum_{\mu \neq \nu} \frac{Z_{\mu} Z_{\nu}}{\left|\mathbf{R}_{\mu}-\mathbf{R}_{\nu}\right|}-\frac{1}{2} \frac{1}{\Omega_{a}} \lim _{\mathrm{G} \rightarrow 0} \frac{4 \pi Z^{2}}{|\mathbf{G}|^{2}},
\end{aligned}
$$

e

$$
\alpha=\frac{1}{\Omega_{a}} \int d \mathbf{r}\left[V_{L}^{p s}(|\mathbf{r}|)+\frac{Z}{|\mathbf{r}|}\right]=\lim _{\mathbf{G} \rightarrow 0}\left[V_{L}^{p s}(\mathbf{G})+\frac{1}{\Omega_{a}} \frac{4 \pi Z}{|\mathbf{G}|^{2}}\right] .
$$

O procedimento de cálculo da energia total se inicia obtendo-se a solução da equação de Kohn-Sham no espaço dos momentos (1.72) para $V_{L}^{p s}(\mathbf{G}=0)$ e $V_{H}(\mathbf{G}=0)$ nulos, e posteriormente são introduzidos os termos $\gamma_{\text {Ewald }}$ e $\alpha$. Assim, a energia total é expressada como:

$$
E_{\text {total }}=E_{c i n}^{(0}+\tilde{E}_{p s}+\tilde{E}_{H}+E_{x c}+\gamma_{E w a l d}+Z \alpha .
$$

Uma expressão mais simples para a energia total do estado fundamental, considerando-se o tratamento dos termos divergentes acima descritos, pode ser obtida de forma análoga à equação (1.20), assim:

$$
\begin{aligned}
E_{\text {total }}= & \sum_{n}^{o c c} E_{n}-\frac{1}{2} \sum_{\mathbf{G} \neq 0} \frac{4 \pi}{|\mathbf{G}|^{2}}|\rho(\mathbf{G})|^{2}+\sum_{\mathbf{G}} \rho(\mathbf{G})\left[\varepsilon_{x c}(\mathbf{G})-\mu_{x c}(\mathbf{G})\right] \\
& +\gamma_{\text {Ewald }}+Z \alpha
\end{aligned}
$$


onde $E_{n}$ são os autovalores obtidos da equação de Kohn-Sham excluíndo-se os termos divergentes. O termo de energia entre os íons, utilizando o método de soma de Ewald, é expressado como:

$$
\begin{aligned}
\gamma_{\text {Ewald }}= & \frac{1}{2} \sum_{\mu} \sum_{\nu} Z_{\mu} Z_{\nu}\left[\frac{4 \pi}{\Omega} \sum_{\mathbf{G} \neq 0} \frac{1}{|\mathbf{G}|^{2}} \cos \left[\mathbf{G} \cdot\left(\mathbf{R}_{\mu}-\mathbf{R}_{\nu}\right)\right] \exp \left(-\frac{G^{2}}{4 \zeta^{2}}\right)\right. \\
& \left.-\frac{\pi}{\zeta^{2} \Omega}+\sum_{\mathrm{L}} \frac{\operatorname{erfc}\left(\zeta\left|\mathbf{L}+\mathbf{R}_{\mu}+\mathbf{R}_{\nu}\right|\right)}{\left|\mathbf{L}+\mathbf{R}_{\mu}+\mathbf{R}_{\nu}\right|}-\frac{2 \zeta}{\sqrt{\pi}} \delta_{\mu, \nu}\right] .
\end{aligned}
$$

onde $\zeta$ é um parâmetro que controla a convergência das somas em $\mathbf{G}$ e $\mathbf{L}$, e $\operatorname{erfc}(x)$ é a função erro complementar.

\subsubsection{Pontos Especiais na Zona de Brillouin}

Na seção anterior vimos que a variável fundamental nos cálculos de energia total e estrutura de bandas é a densidade de carga de valência $\rho(\mathbf{r})$, dadas pelas equações (1.64) e (1.65). Para avaliar esta quantidade seria necessário integrar numericamente em uma malha muito fina de pontos $\mathrm{k}$ na zona de Brillouin, o que é computacionalmente inviável. Embora seja requerida uma integração em toda a zona para se construir a densidade de carga, o grupo de simetria espacial nos permite restringir esta integração na parte irredutível ou primeira zona de Brillouin (ZB), de acordo com as operações de simetria do grupo espacial. Foi baseado nestes argumentos de simetria que Baldereschi $(1972)^{33}$ verificou que a média nos vetores de onda da equação (1.65) podia ser muito bem aproximada por um cálculo considerando um único ponto $\mathrm{k}$, um ponto especial. Posteriormente Chadi e Cohen ${ }^{34}$ e Monkhorst e Pack, ${ }^{45}$ dando continuidade a esta idéia, introduziram métodos para gerar conjuntos de pontos especiais representativos da primeira zona de Brillouin. O ponto de partida nestes métodos foi considerar uma função periódica no espaço recíproco e de variação suave de tal forma a se obter uma média na primeira zona de Brillouin. Esta função $g(\mathbf{k})$, pode ser expandida em série de Fourier:

$$
g(\mathbf{k})=\sum_{\mathbf{R}} g(\mathbf{R}) \exp [i \mathbf{k} \cdot \mathbf{R}]
$$

Assumindo que $g(\mathbf{k})$ possui a simetria completa do cristal, esta também pode ser expandida em ondas planas simetrizadas: 


$$
g(\mathbf{k})=\sum_{m=0}^{\infty} g_{m} A_{m}(\mathbf{k})
$$

com

$$
A_{m}(\mathrm{k})=\frac{1}{n_{\Gamma}} \sum_{\Gamma \in \mathrm{S}}^{m^{*}} \exp [i \mathrm{k} \cdot(\Gamma \mathbf{R})]
$$

onde $m^{*}=\{\Gamma \mathbf{R}\}$ é um conjunto de vetores da rede que se transformam em si mesmos sob as $n_{\Gamma}$ operações de simetria $\Gamma$, do grupo pontual S. Estes conjuntos $m^{*}$, chamados de estrelas, são ordenados de modo que seus comprimentos não diminuem $\left(0<m^{*} \leq(m+1)^{*}\right)$. Duas propriedades importante das funções $A_{m}(\mathrm{k})$ são:

$$
\begin{gathered}
\frac{v}{(2 \pi)^{3}} \int_{Z B} d \mathbf{k} A_{m}(\mathbf{k})=\delta_{m, 0} \\
\frac{v}{(2 \pi)^{3}} \int_{Z B} d \mathbf{k} A_{m}(\mathbf{k}) A_{n}(\mathbf{k})=\frac{1}{N_{m}} \delta_{m, n},
\end{gathered}
$$

onde $N_{m}$ é o número de elementos na $m$-ésima estrela de vetores de translação. Considerando a média na primeira zona de Brillouin da função $g(\mathbf{k})$ da equação (1.105) e aplicando a propriedade (1.107) temos que:

$$
\bar{g}=\frac{v}{(2 \pi)^{3}} \sum_{m=0}^{\infty} g_{m} \int_{Z B} d \mathbf{k} A_{m}(\mathbf{k})=g_{0} .
$$

Assim, o valor médio de $g(\mathbf{k})$ corresponde ao primeiro termo da expansão (1.105). Seja a combinação linear de um conjunto de $N$ pontos $\left\{\mathbf{k}_{i}\right\}$ na primeira zona de Brillouin dada por:

$$
\sum_{i=1}^{N} w_{i} g\left(\mathbf{k}_{i}\right)
$$

Substituindo a expansão (1.105) em (1.110), temos que:

$$
\sum_{i=1}^{N} w_{i} g\left(\mathbf{k}_{i}\right)=\sum_{m=0}^{\infty} g_{m} \sum_{i=1}^{N} w_{i} A_{m}\left(\mathbf{k}_{i}\right) \text {. }
$$

Escolhendo o conjunto $\left\{\mathrm{k}_{i}\right\}$ de forma tal que: 


$$
\sum_{i=1}^{N} w_{i} A_{m}\left(\mathbf{k}_{i}\right)=\delta_{m, 0} \quad \text { para } \quad m<M
$$

Substituindo (1.112) em (1.111) temos que:

$$
\sum_{i=1}^{N} w_{i} g\left(\mathbf{k}_{i}\right)=g_{0}+\sum_{m=M}^{\infty} g_{m} \sum_{i=1}^{N} w_{i} A_{m}\left(\mathbf{k}_{i}\right) .
$$

De acordo com (1.109) e (1.113), o valor médio da função $g(\mathbf{k})$ será:

$$
\bar{g}=g_{0}=\sum_{i=1}^{N} w_{i} g\left(\mathbf{k}_{i}\right)-F,
$$

onde $F$ é a parte residual na fórmula de integração númerica, dada por:

$$
F=\sum_{m=M}^{\infty} g_{m} \sum_{i=1}^{N} w_{i} A_{m}\left(\mathbf{k}_{i}\right)
$$

Logo para uma função arbitrária expressa na forma (1.105), e escolhendo um valor $M$ tal que $g_{0} \neq 0$ e $|F| \ll g_{0}$, o valor médio na primeira zona de Brillouin pode ser aproximado por:

$$
\bar{g} \approx \sum_{i=1}^{N} w_{i} g\left(\mathbf{k}_{i}\right)
$$

Se $g(\mathbf{k})$ é uma função de variação suave, os coeficientes da expansão $g_{m}$ tendem rapidamente a zero quando $m$ cresce. Logo fazendo $M$ suficientemente grande em (1.112) é possível se obter uma boa aproximação para $\bar{g}$.

Aplicando o procedimento acima descrito à função $\rho(\mathrm{k})$, a integral da equação (1.64) pode ser substituída por uma soma nos pontos especiais com um apropriado fator de peso $w_{i}$ tal que $\sum_{i} w_{i}=1$. Desta forma a densidade de carga pode ser reescrita como:

$$
\rho(\mathbf{r})=\sum_{n}^{o c c} \sum_{i} w_{i}\left\langle\rho_{n, \mathbf{k}_{i}}(\mathbf{r})\right\rangle_{\mathrm{S}}
$$

onde $\left\langle\rho_{n, \mathbf{k}_{i}}(\mathbf{r})\right\rangle_{\mathrm{S}}$ é a densidade de carga simetrizada nos pontos especiais $\mathbf{k}_{i}$ com respeito do grupo pontual $\mathrm{S}$, dado por: 


$$
\left\langle\rho_{n, \mathbf{k}_{i}}(\mathbf{r})\right\rangle_{\mathrm{S}}=\frac{1}{n_{\Gamma}} \sum_{\Gamma \in \mathrm{S}} \rho_{n, \Gamma \mathbf{k}_{i}}(\mathbf{r}),
$$

onde $n_{\Gamma}$ é o número de operações $\Gamma$ no grupo pontual S. Finalmente, a componente de Fourier da densidade de carga total de valência calculada nos pontos especiais é expressada como:

$$
\rho(\mathbf{G})=\frac{1}{n_{\Gamma}} \sum_{n}^{o c c} \sum_{i} w_{i} \sum_{\Gamma \in \mathrm{S}} \rho_{n, \Gamma \mathrm{k}_{i}}(\mathbf{G}),
$$

com

$$
\rho_{n, \Gamma \mathbf{k}_{i}}(\mathbf{G})=\frac{1}{N_{c} v} \exp [i \Gamma \mathbf{G} \cdot \mathbf{T}(\Gamma)] \sum_{\mathbf{G}^{\prime}} C_{n}^{*}\left(\mathbf{k}_{i}+\mathbf{G}^{\prime}+\Gamma \mathbf{G}\right) C_{n}\left(\mathbf{k}_{i}+\mathbf{G}^{\prime}\right),
$$

onde $\mathbf{T}(\Gamma)$ é uma translação não primitiva associada a uma dada operação $\Gamma$ do grupo espacial.

\subsubsection{Forças de Hellmann-Feynman}

Se algum dos átomos da célula unitária é deslocado de seu sítio de equilíbrio na estrutura cristalina, então existirá uma força $\mathbf{F}_{\nu}$ atuando no núcleo $\mathbf{R}_{\nu}$. Esta força pode ser calculada tomando-se o gradiente da energia total, isto é:

$$
-\nabla_{\mathrm{R}_{\nu}} E_{\text {total }}=\mathbf{F}_{\nu}
$$

Hellmann $(1937)^{35}$ e Feynman (1939), ${ }^{36}$ mostraram que existem duas contribuições diferentes para a força $\mathbf{F}_{\nu}$. Uma vem da dependência explícita da energia total com respeito às posições dos núcleos $\mathbf{R}_{\nu}$, no termo $E_{i o n, i o n}$. A outra contribuição vem implícita na função de onda $\Phi_{n, \mathbf{k}}(\mathrm{r})$, devido a que esta é solução da equação de Schrödinger monoeletrônica, logo terá uma dependência em $\mathbf{R}_{\nu}$. Assim, a força coulombiana total exercida nos íons é igual ao gradiente negativo do potencial eletrostático devido aos núcleos positivamente carregados (íns) e a densidade de carga eletrônica, logo:

$$
\mathrm{F}_{\nu}=\mathrm{F}_{\nu, i o n}+\mathbf{F}_{\nu, e l} \text {. }
$$


Este resultado é independente da aproximação utilizada para o potencial de troca-correlação. ${ }^{37}$ A força exercida no íon da posição $\mathbf{R}_{\nu}$, devido aos outros íons, é dada por:

$$
\mathbf{F}_{\nu, \text { ion }}=-\nabla_{\mathrm{R}_{\nu}}\left[\sum_{\eta} \sum_{\mu} \frac{Z_{\eta} Z_{\mu}}{\left|\mathbf{R}_{\eta}-\mathbf{R}_{\mu}\right|}\right] .
$$

Utilizando (1.97) obtemos:

$$
\begin{aligned}
\mathbf{F}_{\nu, \text { ion }}= & \frac{1}{2} \sum_{\mu \neq \nu} Z_{\mu} Z_{\nu}\left[\frac{4 \pi}{\Omega} \sum_{\mathbf{G} \neq 0} \frac{\mathbf{G}}{|\mathbf{G}|^{2}} \operatorname{sen}\left[\mathbf{G} \cdot\left(\mathbf{R}_{\mu}-\mathbf{R}_{\nu}\right)\right] \exp \left(-\frac{G^{2}}{4 \zeta^{2}}\right)\right. \\
& \left.+\sum_{\mathrm{L}}\left(\frac{\mathbf{x} \operatorname{erfc}(\zeta x)}{x^{3}}\right)_{\mathbf{x}=\mathbf{L}+\mathbf{R}_{\mu}+\mathbf{R}_{\nu}}\right] .
\end{aligned}
$$

Nesta expressão foi assumido que os íons não se superpõem e são efericamente simétricos. A força exercida nos íons pelos elétrons pode ser calculada diretamente no espaço recíproco, tomando-se o gradiente de $E_{p s}$ na equação (1.90), devido a que a única dependência em $\mathbf{R}_{\nu}$ é dada no fator de estrutura $\mathbf{S}(\mathbf{G})$, equação (1.75). Este fator somente aparece no termo da energia pseudopotencial, assim:

$$
\begin{aligned}
\mathbf{F}_{\nu, e l}= & -\nabla_{\mathrm{R}_{\nu}} E_{p s} \\
= & -\Re\left[i \Omega_{a} \sum_{\mathbf{G}} \rho(\mathbf{G}) \mathbf{G} \exp \left[i \mathbf{G} \cdot \mathbf{R}_{\nu}\right] V_{L}^{p s}(\mathbf{G})\right. \\
& +\frac{i}{N} \sum_{n, \ell, \mathbf{G}, \mathrm{G}^{\prime}} C_{n}^{*}(\mathbf{k}+\mathbf{G}) C_{n}\left(\mathbf{k}+\mathbf{G}^{\prime}\right)\left(\mathbf{G}-\mathbf{G}^{\prime}\right) \\
& \left.\times \exp \left[i\left(\mathbf{G}-\mathbf{G}^{\prime}\right) \cdot \mathbf{R}_{\nu}\right] V_{N L, \ell}^{p s}\left(\mathbf{k}+\mathbf{G}, \mathbf{k}+\mathbf{G}^{\prime}\right)\right],
\end{aligned}
$$

onde $N$ é o número de átomos na célula primitiva. 


\subsection{Dinâmica Molecular de Car-Parrinello}

Na teoria do funcional da densidade, o problema de muitos elétrons, descrito pela equações de Kohn-Sham, é normalmente reduzido a um cálculo de diagonalização de matriz onde, mediante um processo iterativo, é alcançada uma solução autoconsistente. A convergência neste tipo de cálculo é demorada, sendo computacionalmente muito custosa, e estando restrita a sistemas pequenos é ordenados. Car e Parrinello (1985), ${ }^{8}$ introduziram um esquema alternativo de dinâmica molecular de primeiros princípios onde os graus de liberdade eletrônicos podem ser manejados explicitamente, proporcionando assim uma dinâmica atômica estável de uma maneira computacionalmente eficiente. $\mathrm{O}$ procedimento de Car-Parrinello pode ser usado para se calcular diretamente as autofunções de Kohn-Sham a partir de um conjunto de funções de onda de prova. Estas funções de prova sofrem sucessivas "melhorias" em um processo iterativo até que eventualmente convergam nas autofunções de onda do sistema. $^{38}$

O problema da minimização do funcional energia com respeito a densidade de carga eletrônica, nos termos do princípio variacional, pode ser entendido como um processo de procura de valores para certas variáveis eletrônicas que minimizam o funcional energia. Estas variáveis eletrônicas devem definir univocamente a função de onda do sistema. Se consideramos o funcional energia como sendo uma superfície no espaço das variáveis eletrônicas, o ponto mínimo desta superfície corresponderá à energia do estado fundamental. Car e Parrinello introduziram o conceito de resfriamento simulado (simulated annealing) da dinâmica molecular ao problema de muitos elétrons em um sólido. $\mathrm{O}$ resfriamento simulado é um esquema de otimização análogo à forma como as moléculas de um líquido encontram o potencial que proporciona a configuração de mínima energia, quando o líquido é resfriado. A procura de um ponto de mínimo no espaço de configurações eletrônicas é implementado com equações de movimento próprias da dinâmica molecular as quais estão sujeitas a vínculos. Consideremos uma representação de estados eletrônicos em termos de um conjunto de estados ocupados de Kohn-Sham $\left\{\Phi_{1}, \ldots, \Phi_{n}\right\}$. Expandindo cada orbital em uma base arbitrária de funções ortonormais $\left\{\psi_{i}\right\}$ :

$$
\Phi_{i}=\sum_{j} c_{i, j} \psi_{j}
$$

Se considerarmos os coeficientes da expansão como variáveis eletrônicas, logo qualquer configuração eletrônica pode ser completamente especificadas pelo 
conjunto de coeficientes, $\left\{c_{i, j}\right\}$ com $i=1, \ldots, n$ e $j=1, \ldots, m$; onde $n$ é o número de orbitais ocupados e $m$ o número de termos na expansão. No esquema de Car-Parrinello, o funcional energia de Kohn-Sham é uma função do conjunto destes coeficientes, $E\left[\left\{c_{i, j}\right\}\right]$. Cada coeficientes $c_{i, j}$ pode ser considerado como a coordenada de uma "partícula" clássica. Para minimizar este funcional energia, são dadas a estas partículas uma energia cinética e o sistema é gradualmante resfriado até que o conjunto de coordenadas ou coeficientes alcance o valor que minimiza o funcional energia, $\left\{c_{i, j}\right\}_{0}$. Somente nesta configuração o funcional energia terá o significado quântico das equações de Kohn-Sham. Desta forma, o problema de encontrar as funções de onda mono-eletrônicas a partir das equações de Kohn-Sham, é reduzido à solução de um conjunto de equações de movimento clássicas. Escrevendo o funcional energia em função de variáveis dependente do tempo como:

$$
E\left[\left\{\Phi_{i}\right\},\left\{\mathbf{R}_{\nu}\right\},\left\{\alpha_{n}\right\}\right]=\sum_{i} \int d \mathbf{r} \Phi_{i}^{*}(\mathbf{r})\left[-\frac{1}{2} \nabla_{\mathrm{r}}^{2}\right] \Phi_{i}(\mathbf{r})+V\left[\rho(\mathbf{r}),\left\{\mathbf{R}_{\nu}\right\},\left\{\alpha_{n}\right\}\right]
$$

onde $\left\{\mathbf{R}_{\nu}\right\}$ representa as coordenadas nucleares e $\left\{\alpha_{n}\right\}$ definem a forma e dimensão da célula unitária. As equações de movimento do sistema são obtidas na forma usual da mecânica clássica, i.e., a partir de uma lagrangeana:

$$
L=T-V
$$

com

$$
T=\sum_{i} \frac{1}{2} \mu\left\langle\dot{\Phi}_{i} \mid \dot{\Phi}_{i}\right\rangle-\sum_{\nu} \frac{1}{2} M_{\nu} \dot{\mathbf{R}}_{\nu}^{2}+\sum_{n} \frac{1}{2} \mu_{n} \dot{\alpha}_{n}^{2}
$$

e

$$
V=E\left[\left\{\Phi_{i}\right\},\left\{\mathbf{R}_{\nu}\right\},\left\{\alpha_{n}\right\}\right]
$$

onde $\mu$ e $\mu_{n}$ são parâmetros arbitrários que fazem as vezes de "massas", mas que não possuem significado físico real. Estes parâmetros têm a função de controlar a velocidade e o caráter do movimento através do espaço de configurações. $M_{\nu}$ representa a massa dos íons. Desta forma, a energia cinética $T$ possui dois termos fictícios associados aos parâmentros $\mu$ e $\mu_{n}$, e um termo real devido ao movimento dos íons. A condição de ortonormalidade das funções de onda eletrônicas são incorporadas na lagrangeana do sistema na forma de vínculos, por meio do método dos multiplicadores de Lagrange, assim: 


$$
\begin{aligned}
L= & \sum_{i} \frac{1}{2} \mu\left\langle\dot{\Phi}_{i} \mid \dot{\Phi}_{i}\right\rangle+\sum_{\nu} \frac{1}{2} M_{\nu} \dot{R}_{\nu}^{2}+\sum_{n} \frac{1}{2} \mu_{n} \dot{\alpha}_{n}^{2}-E\left[\left\{\Phi_{i}\right\},\left\{\mathbf{R}_{\nu}\right\},\left\{\alpha_{n}\right\}\right] \\
& +\sum_{i, j} \Lambda_{i, j}\left[\left\{\int d \mathbf{r} \Phi_{i}^{*}(\mathbf{r}) \Phi_{j}(\mathbf{r})\right\}-\delta_{i, j}\right] .
\end{aligned}
$$

Os multiplicadores de Lagrange diagonais $\left(\Lambda_{i, i}\right)$ asseguram que a função de onda $\Phi_{i}(\mathbf{r})$ permaneça normalizada, enquanto que os não diagonais $\left(\Lambda_{i, j}\right)$ asseguram que a função de onda permaneça ortogonal. As equações de movimento para cada uma das variáveis da lagrangeana, são obtidas a partir das equações de Lagrange:

$$
\frac{d}{d t}\left(\frac{\partial L}{\partial \dot{x}}\right)-\frac{\partial L}{\partial x}=0
$$

com $x=\left(\left\{\Phi_{i}\right\},\left\{\mathbf{R}_{\nu}\right\},\left\{\alpha_{n}\right\}\right)$, assim as equações de movimento para cada um dos parâmetros dinâmicos são:

$$
\begin{gathered}
\mu \ddot{\Phi}_{i}(\mathrm{r}, t)=-\frac{\delta E}{\delta \Phi_{i}^{*}(\mathbf{r}, t)}+\sum_{j} \Lambda_{i, j} \Phi_{j}(\mathrm{r}, t), \\
M_{\nu} \ddot{\mathbf{R}}_{\nu}=-\nabla_{\mathrm{R}_{\nu}} E \\
\mu_{n} \ddot{\alpha}_{n}=-\frac{\partial E}{\partial \alpha_{n}} .
\end{gathered}
$$

$\mathrm{Na}$ equação (1.133), os multiplicadores de Lagrange adicionam uma força $\Lambda_{i, j} \Phi_{j}$ à força $-\delta E / \delta \Phi_{i}^{*}$. Esta força assegura que a função de onda eletrônica permaneça ortonormal na medida que se propaga ao longo de sua trajetória dinâmica. A equação (1.134) relaciona a aceleração dos íons com a força atuando neles, e a equação (1.135) relaciona a acelaração dos comprimentos dos vetores da rede com as componentes diagonais do tensor de deformação e a aceleração dos ângulos entre os vetores da rede com as componentes não diagonais do tensor de deformação, ambos integrados na célula unitária. Mudando as velocidades dos parâmetros dinâmicos do sistema $\left(\left\{\dot{\Phi}_{i}\right\},\left\{\dot{\mathbf{R}}_{\nu}\right\},\left\{\dot{\alpha}_{n}\right\}\right)$, é possível obter uma diminuição na energia cinética da equação (1.129), e conseqüentemente da temperatura. Assim para $T \rightarrow 0$ o estado de equilíbrio para o valor mínimo do funcional energia é alcançado. Neste ponto a aceleração é nula $\left(\ddot{\Phi}_{i}=0\right)$, portanto a equação (1.133) toma o valor das equações de 
Kohn-Sham e os autovalores da matriz $\Lambda$, coincidem com os autovalores correspondentes aos estados ocupados de Kohn-Sham. Reescrevendo a equação (1.133), obtemos:

$$
\mu \ddot{\Phi}_{i}(\mathbf{r}, t)=-H \Phi_{i}(\mathbf{r}, t)+\sum_{j} \Lambda_{i, j} \Phi_{j}(\mathbf{r}, t),
$$

onde $H$ é a hamiltoniana de Kohn-Sham, definida por:

$$
H \equiv \frac{1}{2} \nabla_{\mathbf{r}}^{2}+\sum_{\nu, \ell} V_{i o n, \ell}^{p s}\left(\mathbf{r}-\mathbf{R}_{\nu}\right)+V_{H}(\mathbf{r})+V_{x c}(\mathbf{r}) .
$$

Se a expansão da equação (1.126) é realizada em um conjunto base de ondas planas, isto é:

$$
\Phi_{i}(\mathbf{r})=\sum_{\mathbf{G}} c_{i}(\mathbf{k}+\mathbf{G}) \exp [i(\mathbf{k}+\mathbf{G}) \cdot \mathbf{r}] .
$$

A equação de movimento (1.136) pode ser escrita no espaço dos momento, onde as variáveis dinâmicas são os coeficientes de expansão. Substituindo (1.138) em (1.136) e integrando em $\mathbf{r}$ obtemos:

$$
\begin{aligned}
\mu \ddot{c}_{i}(\mathbf{k}+\mathbf{G})= & -\left[\frac{1}{2}|\mathbf{k}+\mathbf{G}|^{2}-\sum_{j} \Lambda_{i, j}\right] c_{i}(\mathbf{k}+\mathbf{G}) \\
& -\sum_{\mathbf{G}^{\prime}} V_{e f}\left(\mathbf{k}+\mathbf{G}, \mathbf{k}+\mathbf{G}^{\prime}\right) c_{i}\left(\mathbf{k}+\mathbf{G}^{\prime}\right),
\end{aligned}
$$

onde $V_{e f}$ é o potencial efetivo dado por:

$$
V_{e f}\left(\mathbf{k}+\mathbf{G}, \mathbf{k}+\mathbf{G}^{\prime}\right)=\sum_{\ell} V_{i o n, \ell}^{p s}\left(\mathbf{k}+\mathbf{G}, \mathbf{k}+\mathbf{G}^{\prime}\right)+V_{H}\left(\mathbf{G}-\mathbf{G}^{\prime}\right)+V_{x c}\left(\mathbf{G}-\mathbf{G}^{\prime}\right) .
$$

A evolução dinâmica das funções de onda eletrônicas, dada pela equação de movimento (1.136), considera os íons fixos nas posições $\mathbf{R}_{\nu}$. A dinâmica dos íons é tratada em forma independente a partir da equação (1.134), onde a interação com os elétrons é incluída nas forças de Helmann-Feynman. Estas forças são muito sensíves a erros nas funções de onda $\Phi_{i}(\mathbf{r})$. Isto implica que forças precisas podem ser calculadas unicamente quando as funções de onda estejam muito próximas das verdaderas funções de onda de Kohn-Sham, que é onde o teorema de Hellmann-Feynman tem validade. 


\section{Capítulo 2}

\section{Impurezas Substitucionais de Oxigênio e Nitrogênio em GaAs}

Defeitos nativos têm uma importância fundamental na mediação da difusão de impurezas substitucionais em semicondutores. Estes defeitos, principalmente vacâncias, são comuns em qualquer técnicas de crescimento e sua formação depende fortemente do ambiente no qual o cristal está sendo crescido. Por outro lado, impurezas de átomos leves estão presentes em todas as etapas de fabricação de dispositivos, principalmente nos tratamentos químicos a que estes são submetidos ou em processos de implantação iônica, etc. Estas impurezas tipicamente introduzem níveis profundos na banda proibida que quando elétricamente ativas funcionam como centros de recombinação e captura de elétrons ou buracos, produzindo a degradação de dispositivos. A incorporação de impurezas é um processo inevitável, logo é fundamental conhecer quais são as condições mais favoráveis que determinam sua formação e estabilidade no cristal. Impurezas carregadas reduzem a eficiência da dopagem intrínseca se suas concentrações são comparáveis à dos átomos dopantes. A eficiência da dopagem tipo- $p$ é reduzida pela formação de defeitos positivamente carregados e a dopagem tipo- $n$ é inibida pela formação de defeitos negativos, fenômeno conhecido como compensação. Determinar quais impurezas são mais abundantes e seus estados de carga predominantes nas diferentes condições que um cristal pode ser crescido é a principal motivação para se calcular a energia de formação, para desta forma entender os mecanismos que levam a sua incorporação na estrutura cristalina dos semicondutores.

Neste capítulo apresentaremos cálculos de primeiros princípios da estrutura eletrônica, geometria atômica e energia de formação das impurezas substitucionais de oxigênio e nitrogênio em GaAs. 


\subsection{Oxigênio Substitucional em GaAs}

Oxigênio é um dos contaminantes mais comuns em semicondutores e tem sido detectado em silício, germânio, GaP, GaAs e AlGaAs, em concentrações que vão entre $10^{15}$ e $10^{20} \mathrm{~cm}^{-3}$. O efeito da incorporação de oxigênio em $\mathrm{GaAs}$ tem sido extensivamente estudado do ponto de vista experimental, devido a ser uma impureza eletricamente ativa quando substitui um átomo de arsênio $\left(O_{A s}\right)$, introduzindo um nível semiocupado aproximadamente no meio da banda proibida e fixando o nível de Fermi nesta posição. Apesar do grande esforço colocado para se determinar sua estrutura eletrônica, ainda existem aspectos pouco claros e controvérsias a respeito de suas propriedades. $\mathrm{O}$ interesse neste centro viu-se renovado nos últimos anos devido ao descobrimento de dois modos locais de vibração (LVM), a 731 e $715 \mathrm{~cm}^{-1}$, ${ }^{39}$ que mostram a estrutura fina característica dos isótopos de gálio. Estes modos foram atribuídos às ligações de oxigênio com dois gálios, para diferentes estados de carga, rotulados por A e B. Estudos mais detalhados revelaram a existência de um terceiro estado, rotulado por B' ${ }^{40}$ surgido durante uma conversão opticamente induzida dos estado A ao B. Os estados A, B' e B foram atribuídos ao defeito neutro, negativo e duplamente negativo, respectivamente. $O$ fato dos estados neutro e duplamente negativo serem os únicos detectados em equilíbrio térmico, levou a conclusão de que $O_{A s}$ exibe um comportamento U-negativo, ${ }^{41}$ i.e., um sistema onde o segundo elétron está mais fortemente ligado que o primeiro. Desta forma o estado negativo, associado ao estado de carga intermediario B', não é um estado estável do sistema. Apesar da distorção da rede não ser acessível com a espectroscopia de modos vibracionais, os autores propuseram, baseados nos modos locais observados, um deslocamento do oxigênio com respeito ao sítio do arsênio na direção $\langle 100\rangle$, formando uma ligação com dois gálios primeiros vizinhos do tipo $G a-O-G a$, enquanto que os dois gálios restantes se ligariam entre $\mathrm{si}^{7}{ }^{7}$ de forma similar ao centroA em silício. ${ }^{5}$ Esta distorção ocorreria nos três estados de carga observados. Recentemente medidas de dicroismo circular magnético da absorção óptica $(\mathrm{MCDA})^{42,43}$ perto do infravermelho, confirmaram a existência de um estado fundamental paramagnético associado ao estado B'. Isto mostraria que o estado instável que exibe o comportamento U-negativo para o defeito $O_{A s}$, seria um estado paramegnético.

Um dos primeiros estudos teóricos do oxigênio substituindo um arsênio em $G a A s$, consiste em um cálculo de cluster onde os orbitais atômicos são descritos por funções gaussianas. ${ }^{44}$ Neste cálculo é encontrado que a impureza $O_{A s}$ pode existir em cinco diferentes estados de carga (de 1+ a 3-) onde o estado 
neutro é instável. Também é observado um grande deslocamento do oxigênio na direção $\langle 100\rangle$ para os estados de carga 1-, 2- e 3-, concordando parcialmente com os resultados experimentais. Estudos teóricos que utilizam métodos de energia total de primeiros princípios têm permanecido proibitivos devido a dificuldade que apresentam na descrição do átomo de oxigênio. A forte localização de suas funções de onda eletrônicas fazem necessário uma expansão com um grande número de termos, especialmente quando é utilizado um conjunto de ondas planas. Este problema tem sido recentemente contornado graças ao surgimento de uma nova geração de pseudopotenciais suaves que tem permitido diminuir o número de ondas planas necessárias para sua correta descrição, fazendo que estes cálculos sejam computacionalmente acessíveis.

Nossos cálculos para oxigênio em $G a A s$ foram realizados no formalismo do funcional da densidade $(\mathrm{DFT})^{9}$ onde a energia total é obtida resolvendo-se as equações de Kohn-Sham em forma autoconsistente. Para descrever o potencial de troca-correlação é utilizada a aproximação da densidade local (LDA) ${ }^{18}$ Os átomos foram descritos por pseudopotenciais suaves de norma conservada, gerados pelo método de Troullier-Martins. ${ }^{27}$ Os cálculos são realizados no espaço recíproco, onde as funções de onda são expandidas em um conjunto de ondas planas até uma energia cinética equivalente a uma energia de corte de $48 \mathrm{Ry}$, e a integração na primeira zona de Brillouin é obtida pelo esquema dos pontos especiais, onde foram utilizados dois pontos- $k$ de Monkhorst-Pack. ${ }^{45}$ A estrutura cristalina foi simulada por meio de uma supercélula cúbica $b c c$ de 32 átomos onde a impureza é colocada no centro da célula, e são descongeladas até duas camadas atômicas em volta da impureza para encontrar a configuração de equilíbrio. A geometria atômica é obtida conjuntamente com o estado fundamental eletrônico do sistema por meio do cálculo das forças de Hellmann-Feynman ${ }^{35,36}$ utilizando o esquema de dinâmica molecular de Car-Parrinello, ${ }^{8}$ implementado em um código otimizado. ${ }^{46}$ As forças foram consideradas convergidas para um valor de $0.05 \mathrm{eV} / \AA$.

\subsubsection{Oxigênio Substituindo um Arsênio em GaAs}

Nossos resultados para a estrutura eletrônica da impureza de oxigênio substituindo um átomo de arsênio em $G a A s$, no estado de carga neutro $\left(O_{A s}^{0}\right)$, mostram um nível de simetria $a_{1}$, ocupado com um elétron, aproximadamente no meio do gap e um nível de simetria $t_{2}$, vazio, ressonante no fundo da banda de condução. Estes níveis surgidos de uma hibridização entre os orbitais atômicos da impureza substitucional de oxigênio e os quatro orbitais híbridos 


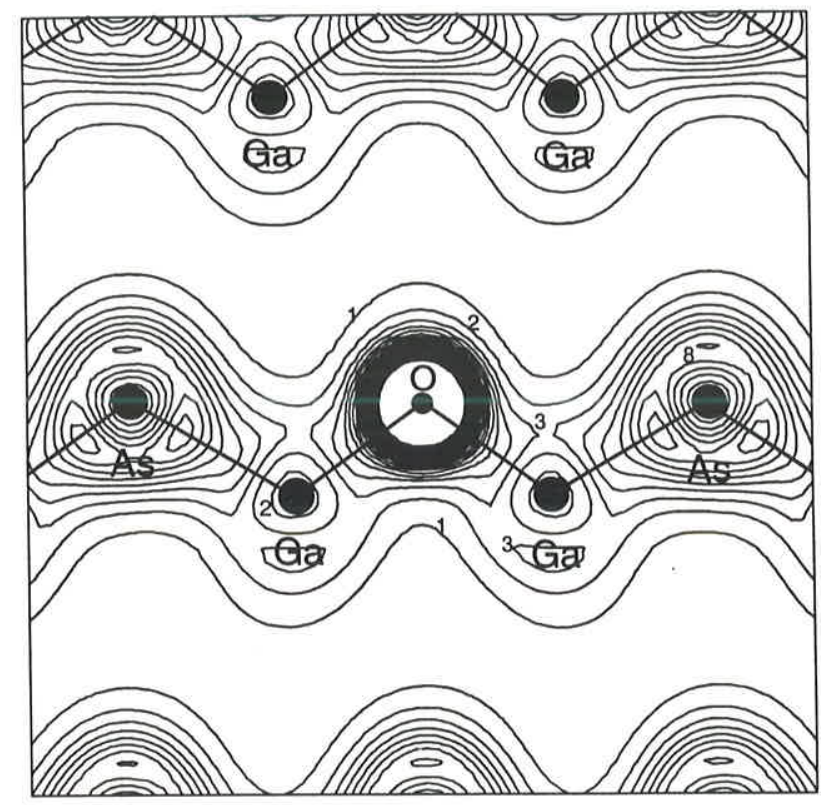

Figura 2.1: Gráfico de contorno da densidade de carga total de valência no plano [110] para a impureza de oxigênio substituindo um átomo de arsênio em GaAs no estado de carga neutro $\left(O_{A s}^{0}\right)$. Unidades em 11.3 eletrons/volume da célula $(e / \Omega)$.

flutuantes da vacância de arsênio, estão localizados a $0.67 \mathrm{eV}$ e $1.32 \mathrm{eV}$, acima do topo da banda de valência, respectivamente. O valor do gap teórico foi calculado em $1.30 \mathrm{eV}$. Com respeito a estrutura geométrica, não foi observado nenhum deslocamento considerável da impureza em relação ao sítio substitucional.

Para verificar a possibilidade de um estado metaestável neste sítio, calculamos a energia total para diferentes posições do oxigênio ao longo das direções $\langle 100\rangle$ e $\langle 111\rangle$. O oxigênio foi fixado em diferentes posições ao longo destas direções, enquanto que seus primeiros e segundos vizinhos foram permitidos de relaxar. Nossos resultados mostram que o sistema de menor energia tem o oxigênio ocupando o sítio substitucional do arsênio. É observada uma relaxação dos quatro gálios primeiros vizinhos na direção da impureza, resultando em uma diminuição da distância de ligação $G a-O$ da ordem de $12 \%$ com respeito à distância de ligação $G a-A s$ no cristal puro $(2.43 \AA)$. A distância de ligação entre os gálios primeiros vizinhos e os arsênios segundos vizinhos aumenta em $2.5 \%$. Esta relaxação é acompanhada por uma diminuição na energia total do sistema de $1.10 \mathrm{eV}$. 

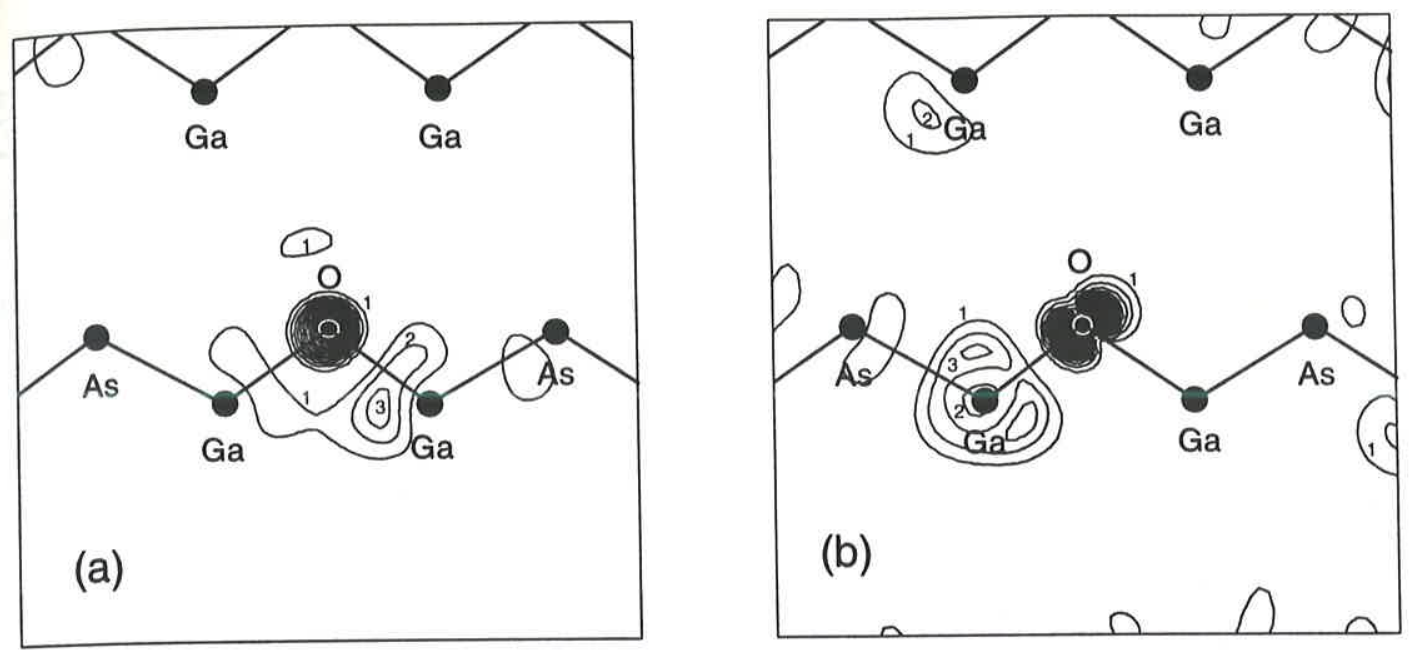

Figura 2.2: Gráficos de contorno da densidade de carga do último estado ocupado (a) e primeiro estado vazio (b), no plano [110], para o defeito $O_{A s} \mathrm{em}$ GaAs no estado de carga neutro. Unidades em $0.11 \mathrm{e} / \Omega$.

Na Figura 2.1 é mostrado o gráfico de contorno da densidade de carga total de valência no plano [110] para este centro. Aqui vemos que oxigênio conserva sua característica atômica acumulando uma grande quantidade de carga em sua volta e ligando-se fracamente com os gálios primeiros vizinhos. Na Figura 2.2(a) mostramos o gráfico de contorno da densidade de carga do último nível ocupado $\left(a_{1}\right)$, localizado no gap. Este apresenta um forte caráter tipo $s$ na impureza mostrando uma hibridização praticamente nula com os orbitais associados à vacância. Na Figura 2.2(b) mostramos a densidade de carga do primeiro nível vazio $\left(t_{2}\right)$ localizado ressonante no fundo da banda de condução. Este também apresenta um forte caráter atômico, tipo $p$, o qual mostra uma distribuição de carga alinhado na direção de um dos gálios primeiros vizinhos, mostrando características antiligante. A Figura 2.2 mostra que oxigênio mantém sua característica atômica quando ocupa um sítio de arsênio em $G a A s$, onde seus orbitais praticamente não hibridizam com os orbitais flutuantes da vacância. Estes orbitais flutuantes apresentam uma distribuição de carga espalhada entre o oxigênio e os quatro gálios primeiros vizinhos, Figura 2.2(a).

Considerando os resultados experimentais que prevêem um deslocamento do oxigênio na direção $\langle 100\rangle$, calculamos a superfície de energia total como função do deslocamento desta impureza nas direções $\langle 100\rangle$ e $\langle 111\rangle$, para os estados de carga 1- e 2-. Nossos resultados podem ser vistos na Figura 2.3, 

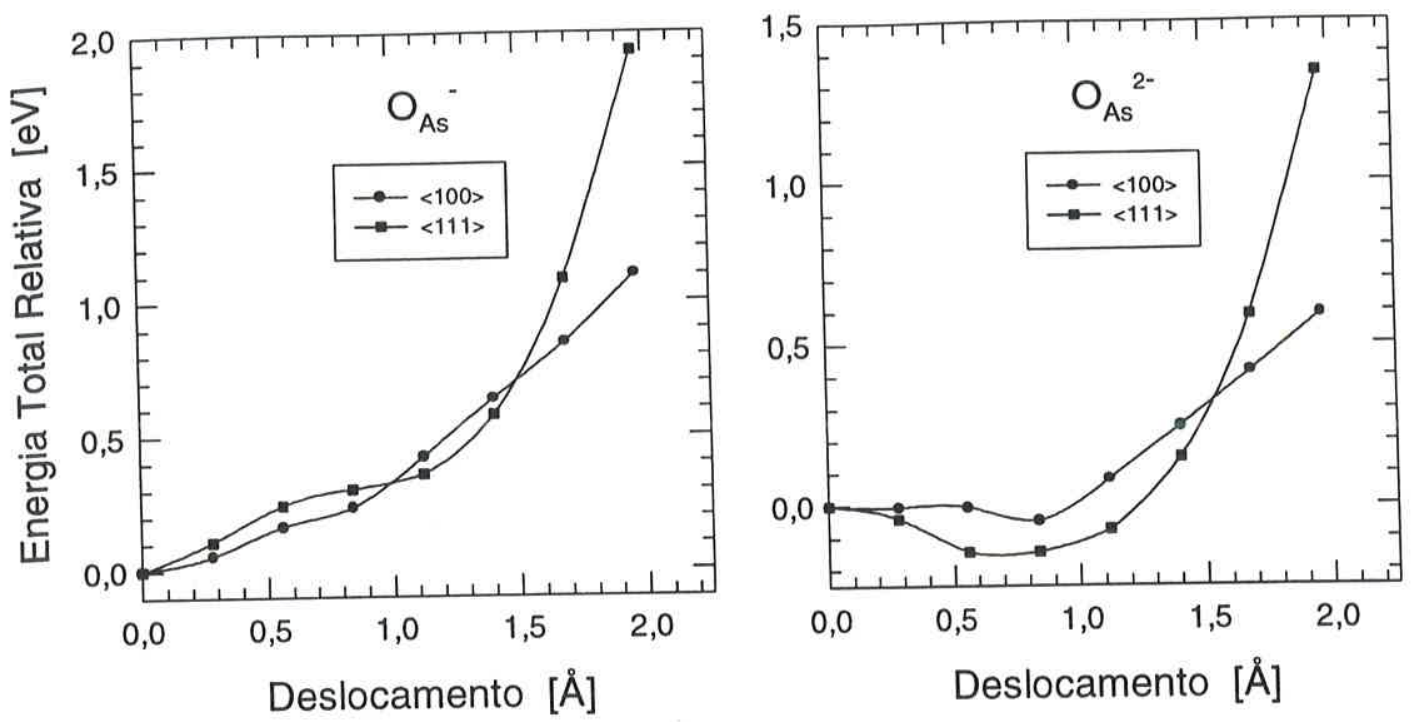

Figura 2.3: Energia total relativa como função do deslocamento do átomo de oxigênio nas direções $\langle 100\rangle$ e $\langle 111\rangle$ para os sistemas de impureza $O_{A s}^{-}$e $O_{A s}^{2-}$ em GaAs. A energia e deslocamento zero correspondem ao sítio tetraédrico.

onde o deslocamento zero se refere ao sítio substitucional do arsênio. Vemos claramente que para o sistema $O_{A s}^{-}$o sítio mais estável é o substitucional, demonstrando a não existência de deslocamentos. Neste estado de carga o nível $a_{1}$ do gap, agora ocupado com dois elétrons, e o nível $t_{2}$, vazio, mantém praticamente as mesmas posições em relação ao sistema neutro. Para o sistema $O_{A s}^{2-}$, deslocando o oxigênio na direção $\langle 111\rangle$, observamos um mínimo de energia aproximadamente a $0.7 \AA$ do sítio substitucional, provocando uma diminuição da energia total do sistema de $0.14 \mathrm{eV}$. Também observamos uma uma posição de metaestabilidade na direção $\langle 100\rangle$ aproximadamente a $0.8 \AA$ do sítio substitucional. A diminuição da energia total neste caso é de $0.05 \mathrm{eV}$. Estes deslocamentos da impureza podem ser explicados como distorções Jahn-Teller, ${ }^{47}$ na qual um sistema localizado em um estado com degenerecência orbital (aqui referido como estado ou nível degenerado) distorce espontâneamente reduzindo sua simetria até que o estado resultante não apresente tal degenerecência. Para nosso sistema de defeito $O_{A s}^{2-}$, o último nível ocupado em simetria tetraédrica é tridegenerado, logo no estado fundamental a degenerecência deve ser removida distorcindo a rede. A simetria do sistema abaixa de $T_{d}$ para $C_{3 v}$, produzindo uma quebra dos níveis degenerados $\left(t_{2} \rightarrow a_{1}+e\right)$. Na situação de mínima energia observamos três níveis no gap, um nível $a_{1}$ ocupado com dois elétrons que corresponde ao originalmente observado no gap, e outros dois níveis, $a_{1}$ e $e$, 

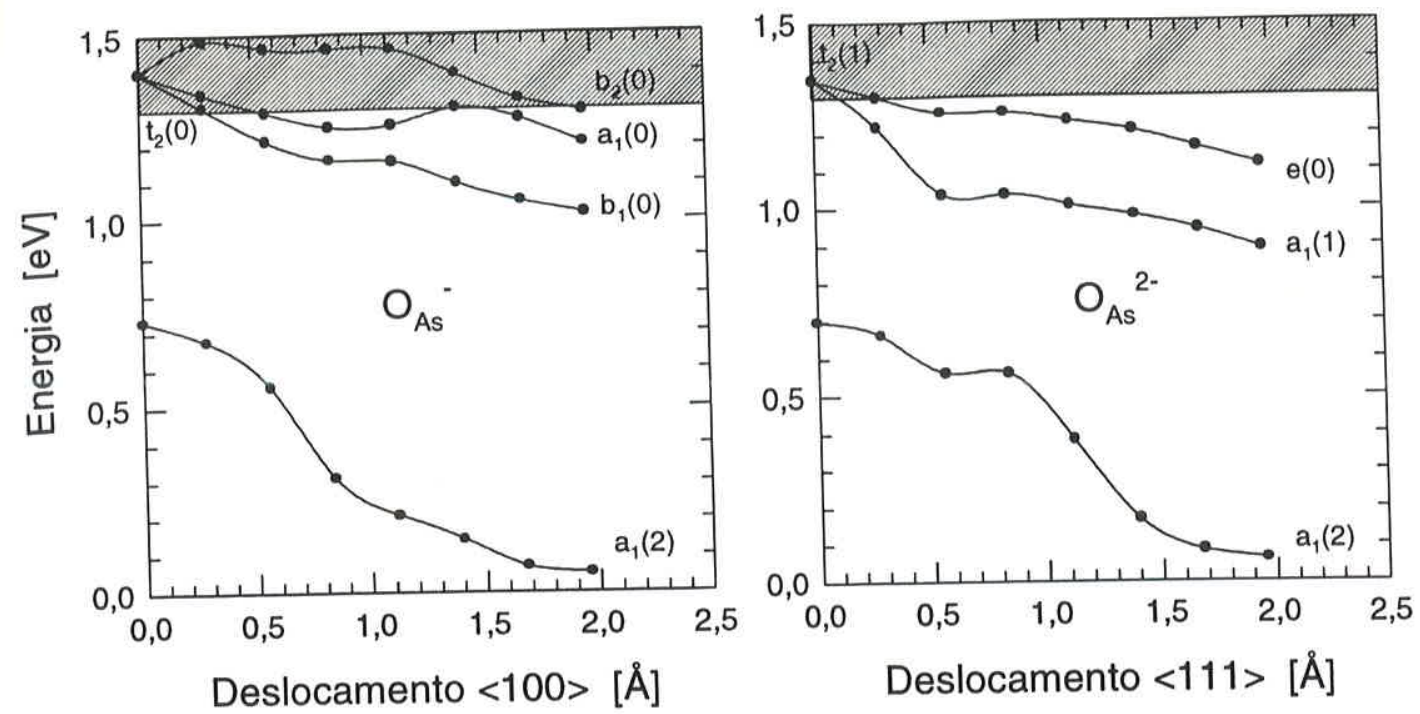

Figura 2.4: Autovalores de Kohn-Sham correspondentes aos niveis no gap, em função do deslocamento do oxigênio, para os sistemas $O_{A s}^{-}$na direção $\langle 100\rangle e$ $O_{A s}^{2-}$ na direção $\langle 111\rangle$. Entre parêntesis é indicada a ocupação dos níveis. A região hachurada representa a banda de condução.

ocupado com um elétron e vazio respectivamente, produto do desdrobramento do nível $t_{2}$. O nível $a_{1}$ duplamente ocupado mantém a mesma posição no gap, os outros dois níveis se localizam aproximadamente a $1.1 \mathrm{e} 1.3 \mathrm{eV}$ acima do topo da banda de valência respectivamente.

Para verificarmos a não existência de distorções no sistema negativo $O_{A s}^{-}$, desenhamos a variação dos níveis no gap como função do deslocamento na direção $\langle 100\rangle$, e comparamos com a variação dos mesmos níveis para o sistema duplamente negativo $O_{A s}^{2-}$, na direção $\langle 111\rangle$, onde é observada uma distorção. Os resultados são mostrados na Figura 2.4. Aqui vemos que para o sistema $O_{A s}^{-}$, o deslocamento na direção $\langle 100\rangle$ produz a remoção da degenerecência do nível $t_{2}$ desdobrando-o em três níveis não degenerados: $a_{1}, b_{1}$ e $b_{2}$, devido à redução da simetria do sistema, de $T_{d}$ para $C_{2 v}$. Para o sistema $O_{A s}^{2-}$, o deslocamento é na direção $\langle 111\rangle$, logo a simetria é reduzida agora para $C_{3 v}$, e assim o nível $t_{2}$ desdobra-se em dois níveis, um $a_{1}$ ocupado com um elétron e um $e$, vazio. Nos dois sistemas observamos que os níveis associados ao $t_{2}$ no sítio substitucional entram no gap quando a impureza de oxigênio é deslocada, enquanto que o nível $a_{1}$, no meio do gap, desce até o topo da banda de valência. No sistema $O_{A s}^{2-}$, é observada uma aproximação entre os dois níveis $a_{1}$, sendo máxima para um deslocamento de $0.7 \AA$ que é exatamente a posição 


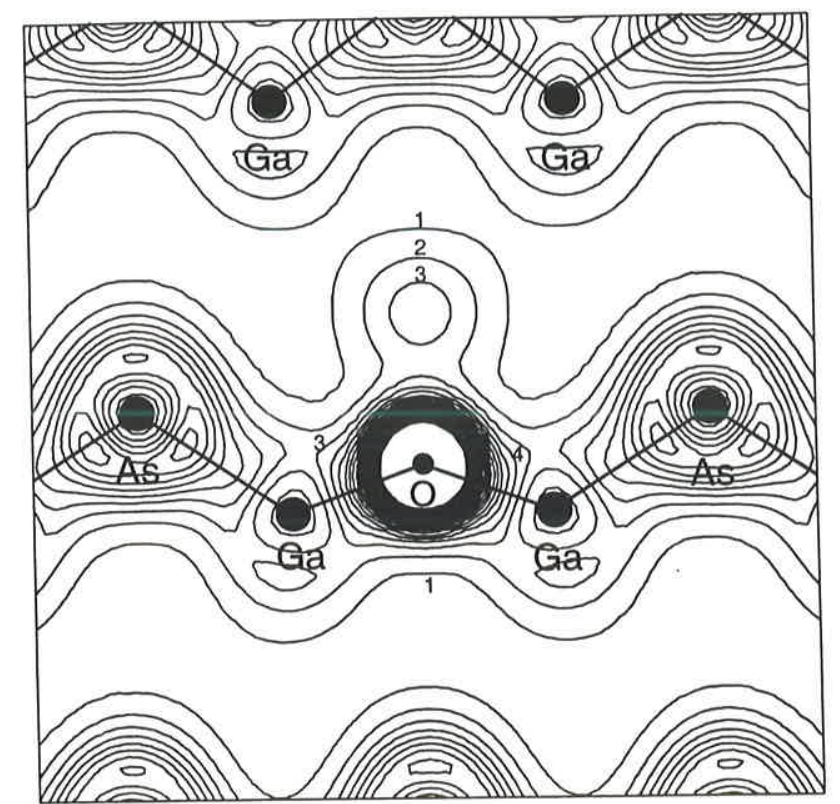

Figura 2.5: Gráfico de contorno da densidade de carga total de valência no plano [101] para a impureza de oxigênio substituindo um átomo de arsênio em GaAs no estado de carga triplamente negativo, $\left(O_{A s}^{3-}\right)$. Unidades em 13.0 e/ $\Omega$.

onde o sistema apresenta a mínima energia total, Figura 2.3. Este tipo de comportamento dos níveis no gap é característico dos sistemas de impureza que apresentam deslocamento. Se compararmos com o sistema $O_{A s}^{-}$, vemos que não existe uma interação semelhante entre o nível $a_{1}$ duplamente ocupado e algum dos níveis superiores, que estão vazios. Logo, o sistema $O_{A s}^{-}$em $G a A s$, cuja estrutura eletrônica é similar ao sistema $O_{S i}^{0}$ em silício (centro-A), não apresenta a distorção observada neste último a qual é atribuida a um efeito pseudo-Jahn-Teller. ${ }^{48,49}$

Com o objetivo de verificar a geometria atribuida à impureza de oxigênio em GaAs nos resultados experimentais, realizamos cálculos de energia total para este centro no estado de carga triplamente negativo $\left(O_{A s}^{3-}\right)$. A configuração de mínima energia mostra uma grande distorção do átomo de oxigênio na direção $\langle 0 \overline{1} 0\rangle$, deslocando-se $0.79 \AA$ do sítio substitucional e produzindo uma diminuição na energia total de $2.11 \mathrm{eV}$. A geometria de equilíbrio mostra o oxigênio próximo a dois gálios formando uma ligação $G a-O-G a$ como vista experimentalmente. O ângulo entre estas ligações é de $141.6^{\circ}$ e a distância de ligação $G a-O$ é de $1.89 \AA$, para ambos os pares. Os outros dois gálios segundos vizinhos ficam mais afastados do oxigênio, a 2.39 e $2.49 \AA$ respectiva- 


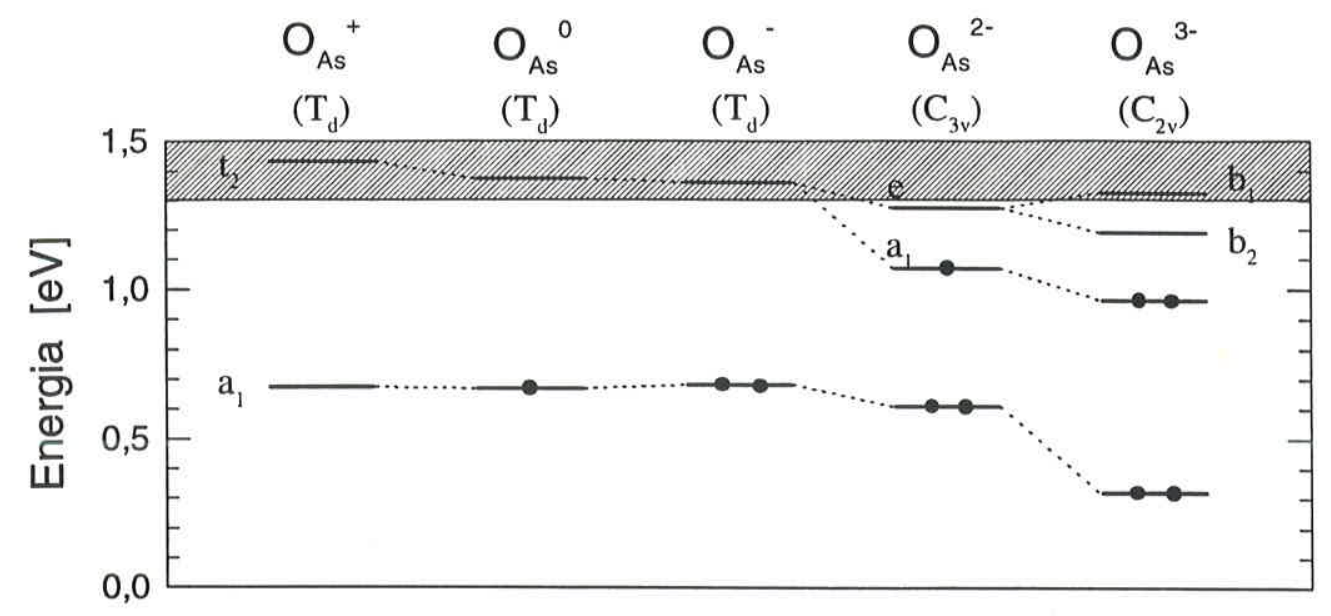

Figura 2.6: Representação esquemática dos autovalores de Kohn-Sham associados aos niveis de defeito no gap para o centro $O_{A s}$ em GaAs, nos cinco estados de carga estudados. Entre parêntesis é indicada a simetria de equilibrio de cada sistema. Os círculos cheios indicam a ocupação nos níveis e a região hachurada representa o fundo da banda de condução.

mente, formando uma ligação $G a-G a$ de $2.76 \AA$ de comprimento. Na Figura 2.5 mostramos o gráfico de contorno da densidade de carga total no plano [101] para este sistema. Aqui vemos claramente a formação da ligação $G a-O-G a$ que conserva a mesma característica das ligações tetraédricas. Na parte superior da distribuição de carga do oxigênio se observa a seção transversal da ligação $G a$ $G a$ no plano [101], onde notamos que esta ligação não tem uma participação com o oxigênio. A estrutura eletrônica do centro $O_{A s}^{3-}$ no estado de equilíbrio, mostra três níveis no gap, dois níveis $a_{1}$ totalmente ocupados e um nível $b_{1}$ vazio, localizados a $0.32,0.96$ e $1.19 \mathrm{eV}$ acima do topo da banda de valência, respectivamente. Um quarto nível vazio é observado ressonante no fundo da banda de condução. Na Figura 2.6 apresentamos esquematicamente a posição dos níveis de impureza observados na banda proibida do centro $O_{A s}$ para os diferentes estados de carga estudados, nas respectivas geometrias de equilíbrio. Pelo fato de serem obtidos em cálculos de supercélula, eles estão sujeitos a um erro intríseco dado pela dispersão, da ordem de $0.1 \mathrm{eV}$. Na Figura 2.6 vemos que para os estados de carga positivo, neutro e negativo não é observada nenhuma variação na posição dos níveis de impureza $\left(a_{1}\right.$ e $\left.t_{2}\right)$, que é coerente com a não distorção observada nestes sistemas. Para os estados de carga 2-, a distorção da rede produz a redução da simetria $\left(T_{d} \rightarrow C_{3 v}\right)$, produzindo o desdobramento do nível $t_{2}\left(a_{1}+e\right)$ e observam-se dois níveis $a_{1}$, ocupados com 
Tabela 2.1: Geometria de equilíbrio da primeira vizinhança do defeito $O_{A s} \mathrm{em}$ GaAs para os cinco estados de carga estudados. $d_{i}$ representa a distância de ligação entre os quatro gálios primeiros vizinhos e a impureza $\left(G a_{i}-O\right)$ e $\Delta E_{r}$ a energia de relaxação do sistema.

\begin{tabular}{|c|c|c|c|c|c|}
\hline Defeito & $d_{1}[\AA]$ & $d_{2}[\AA]$ & $d_{3}[\AA]$ & $d_{4}[\AA]$ & $\Delta E_{r}[\mathrm{eV}]$ \\
\hline \hline$O_{A s}^{+}$ & 2.19 & 2.17 & 2.18 & 2.19 & 0.78 \\
\hline$O_{A s}^{0}$ & 2.15 & 2.13 & 2.15 & 2.15 & 1.10 \\
\hline$O_{A s}^{-}$ & 2.13 & 2.10 & 2.12 & 2.12 & 1.46 \\
\hline$O_{A s}^{2-}$ & 1.98 & 2.72 & 1.98 & 1.98 & 1.65 \\
\hline$O_{A s}^{3-}$ & 1.89 & 2.49 & 2.39 & 1.89 & 2.11 \\
\hline
\end{tabular}

um e dois elétrons, e com diferença de energia entre eles de $0.46 \mathrm{eV}$. Para o estado de carga 3-, a simetria volta a se reduzir $\left(C_{3 v} \rightarrow C_{2 v}\right)$ produzindo o desdobramento do nível $e\left(b_{1}+b_{2}\right)$ e observando-se os mesmos dois níveis $a_{1}$ agora totalmente ocupados. Neste caso a diferença em energia entre eles é maior, $0.64 \mathrm{eV}$, devido a uma maior repulsão eletrônica.

Na Tabela 2.1 apresentamos um resumo da geometria de equilíbrio obtida para o defeito $O_{A s}$ em GaAs nos diferentes estados de carga estudados. São mostradas as distâncias de ligação entre os quatro gálios primeiros vizinhos e a impureza na configuração de mínima energia, e também é mostrada a energia de relaxação envolvida em cada sistema. Notamos que o valor mínimo da energia de relaxação é obtido no estado de carga positivo onde a impureza é isovalente com respeito ao arsênio que está substituindo. Ao colocar elétrons no sistema, a energia de relaxação aumenta devido à criação de ligações flutuantes, as quais interagem eletrostaticamente com as ligações atômicas $O-G a$, tornando-as mais fracas e finalmente produzindo sua quebra. A incorporação de um elétron ao sistema, produz um energia de relaxação entre 0.2 e $0.4 \mathrm{eV}$. A grande distorção observada no sistema $O_{A s}^{3-}$ é consoante com a maior energia de relaxação observada.

O cálculo da energia de formação de um defeito, permite obter informação a respeito das condições mais favoráveis que posibilitam sua incorporação na rede, como também determinar os pocessos dominantes entre diferentes sistemas de defeitos em competição. Considerando o cristal como sendo um sistema termodinâmico de múltiples componentes, a energia de formação é descrita em termos das abundâncias relativas dos átomos constituentes em equilíbrio termodinâmico. Estas abundâncias são representadas pelos poten- 
ciais químicos que constituem as variáveis do sistema. Para a impureza de oxigênio substituindo um arsênio em $\operatorname{GaAs}\left(O_{A s}\right)$, no estado de carga $q$, a energia de formação pode ser escrita como, ${ }^{50}$

$$
E_{\text {form }}\left(O_{A s}^{q}\right)=E_{\text {total }}\left(O_{A s}^{q}\right)-n_{G a} \mu_{G a}-n_{A s} \mu_{A s}-\mu_{O}-q \mu_{e}
$$

onde $E_{\text {total }}\left(O_{A s}^{-q}\right)$ é a energia total calculada com a supercélula, $n_{G a}\left(n_{A s}\right)$ representa o número de átomos de $G a(A s)$ na célula e $\mu_{e}$ é o potencial químico eletrônico (posição do nível de Fermi). Os potencias químicos de gálio e arsênio não são variáveis independentes, eles estão vinculados pela condição de estabilidade do cristal $\left(\mu_{G a}+\mu_{A s}=\mu_{G a A s(b u l k)}\right)$, que deve ser mantida para assegurar a condição de equilíbrio. Por outro lado $\mu_{G a}$ e $\mu_{A s}$ variam em um intervalo dado pelo calor de formação do $G a A s$, onde o limite superior é dado pelas suas respectivas fases bulk. Se considerarmos $\mu_{G a}$ ou $\mu_{A s}$ como sendo igual a sua respectiva fase bulk na equação (2.1), é possível obter a energia de formação do defeito como incorporado em um ambiente de crescimento extremo, rico em átomos de gálio ou arsênio, sendo possível simular uma condição tipicamente experimental. Para estabelecer um limite superior para o potencial químico da impureza, é necessário considerar outras possíveis fases cristalinas ou mesmo moléculas que ela poderia formar com os átomos na qual se encontra ligada no sistema de defeito. Para nosso defeito $O_{A s}$, o valor máximo para $\mu_{O}$ foi obtido a partir do cálculo da energia total do óxido de gálio $\mathrm{Ga}_{2} \mathrm{O}_{3}$, e maiores detalhes deste cálculo são apresentados no Apêndice D.

Calculamos a energia de formação da impureza $O_{A s}$ em $G a A s$ para um ambiente simulado rico em gálio, que é a situação mais favorável para a incorporação de defeitos do tipo $X_{A s}$. O intervalo de variação do potencial químico eletrônico é considerado como sendo o valor experimental da banda proibida do GaAs (1.52 eV). Nossos resultados são mostrados na Figura 2.7. A partir destes resultados podemos concluir que a impureza $O_{A s}$ em GaAs pode existir em cinco diferentes estados de carga (de 1+ até 3-). Os níveis de ocupação ou estados de transição são definidos como sendo iguais ao potencial químico eletrônico (nível de Fermi) para o qual o defeito em dois estados de carga apresentam a mesma energia de formação. Os estados de transição podem ser obtidos diretamente da Figura 2.7 procurando-se a interseção de duas linhas de estados de carga consecutivas. Desta forma o nível doador ou estado de transição para a transição $+\rightarrow 0$ é encontrado a $0.24 \mathrm{eV}$ acima do topo da banda de valência, que denotamos como $E(0 /+)=E_{v}+0.24 \mathrm{eV}$. Da mesma forma os níveis aceitadores são localizados a $E(-/ 0)=E_{v}+0.43 \mathrm{eV}, E(2-/-)$ 


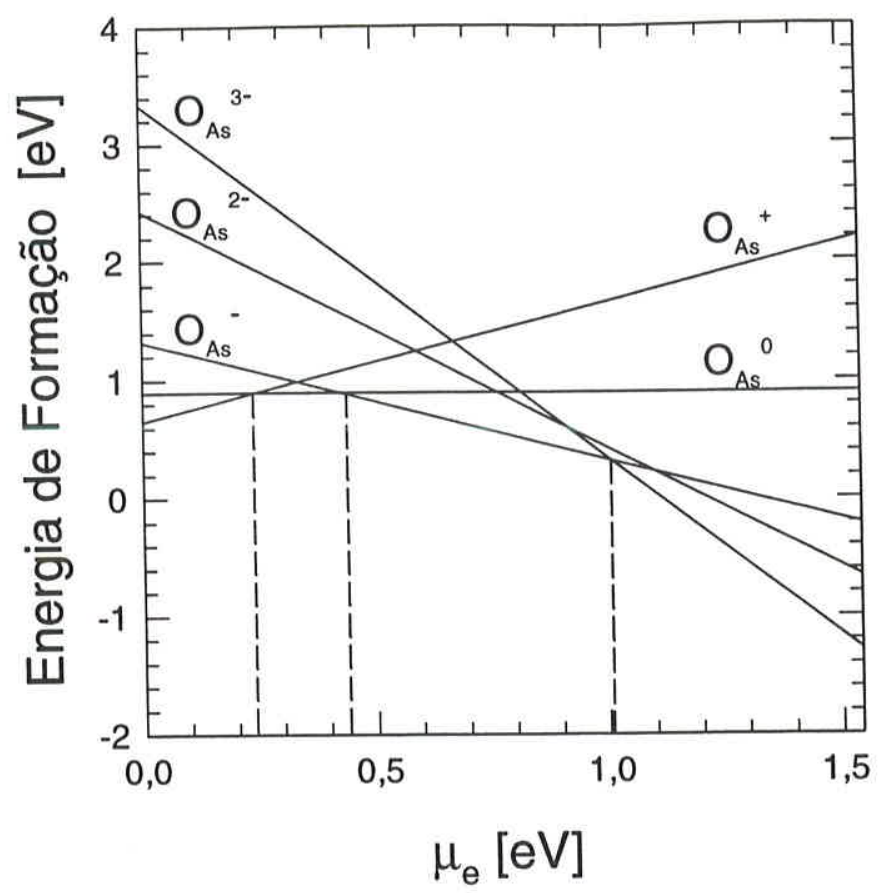

Figura 2.7: Energia de formação do defeito $O_{A s}$ em GaAs como função do potencial químico eletrônico $\left(\mu_{e}\right)$ ou nivel de Fermi, para um ambiente simulado rico em gálio $\left(\mu_{G a}=\mu_{G a(b u l k)}\right)$. As linhas tracejadas representam a posição dos estados de transição: $(0 /+)=0.24 \mathrm{eV},(-/ 0)=0.43 \mathrm{eV}$ e $(3-/-)=1.01 \mathrm{eV}$.

$=E_{v}+1.01 \mathrm{eV}$ e $E(3-/ 2-)=E_{v}+0.91 \mathrm{eV}$. Devido ao fato do aceitador duplo (2-/-) ser encontrado acima do aceitador triplo (3-/2-) na banda proibida, mostrando que o sistema com três elétrons está mais fortemente ligado que o com dois elétrons, concluimos que o centro $O_{A s}$ em $\mathrm{GaAs}$ exibe um comportamento U-negativo, onde a energia de correlação de Hubbard é $|\mathrm{U}|=0.19 \mathrm{eV}$. Desta forma o sistema $O_{A s}^{2-}$ não é estável, dando lugar a uma transição que envolve dois elétrons, e o estado de transição associado se localiza em $E(3-/-)$ $=E_{v}+1.01 \mathrm{eV}$. A propriedade U-negativo observada no nosso defeito, que é evidenciada no ordenamento incomum entre os níveis aceitadores (2-/-) e (3/2-), pode ser explicada em termos das distorções Jahn-Teller observadas nos sistemas $O_{A s}^{2-}$ e $O_{A s}^{3-}$. Devido à energia de relaxação ser maior para o estado de carga 3- do que no 2-, a diferença entre elas $(\sim 0.46 \mathrm{eV})$ compensa a energia necessária para ultrapassar a energia de repulsão coulombiana surgida ao se adicionar o terceiro elétron, produzindo uma interação eletrônica atrativa no sítio do defeito, i.e., o comportamento U-negativo. 
Voltando a Figura 2.7 notamos que para GaAs tipo- $p$, no qual o nível de Fermi está situado perto do topo da banda de valência, a impureza $O_{A s}$ deveria ser encontrada nos estados de carga positivo e neutro, que apresentam uma menor energia de formação. Para GaAs tipo-n, com o nível de Fermi perto do fundo da banda de condução, a situação mais favorável é encontrada com a impureza no estado de carga triplamente negativo. Finalmente para GaAs não dopado intencionalmente, é mais provável se encontrar a impureza no estado de carga negativo. É importante notar que quando o nível de Fermi ultrapassa o valor de $1.10 \mathrm{eV}$, a energia de formação do sistema $O_{A s}^{3-}$ se torna negativa. Este resultado implica que na condição rica em gálio, que é a que estamos supondo para nosso defeito, não seria possível se mover o nível de Fermi por sobre $1.10 \mathrm{eV}$ e manter o equilíbrio termodinâmico.

Como resumo desta seção podemos dizer que, de acordo com nossos resultados, a impureza de oxigênio substituindo um arsênio em GaAs pode existir em cinco estados de carga (de 1+ até 3-), onde o estado de carga 2- é instável. Desta forma o centro $O_{A s}$ apresenta um comportamento Unegativo, observando-se três estados de transição no gap: $(0 /+),(-/ 0)$ e (3/2-). Nos sistemas $O_{A s}^{+}, O_{A s}^{0}$ e $O_{A s}^{-}$não foram observados deslocamentos da impureza com respeito ao sítio substitucional do arsênio. O sistema $O_{A s}^{2-}$, na configuração de mínima energia, apresenta um deslocamento do oxigênio de $0.7 \AA$ na direção $\langle 111\rangle$. Também é observado um outro deslocamento na direção $\langle 100\rangle$, mas energeticamente menos favorável. Para o sistema $O_{A s}^{3-}$, o estado de equilíbrio mostra um deslocamento do oxigênio na direção $\langle 100\rangle$ de $0.79 \AA$, onde é observada a formação da ligação $G a-O-G a$.

Com respeito a outros resultados teóricos, recentemente foi publicado um cálculo de primeiros princípios onde é estudado oxigênio em GaAs, GaN e $A l N .^{51}$ Nestes cálculos os sistemas são descritos com dois tipos diferentes de pseudopotenciais, um de norma não conservada ${ }^{52}$ para descrever a impureza de oxigênio e um outro de norma conservada ${ }^{53}$ para os átomos hospedeiros. Os resultados para o defeito $O_{A s}$ em GaAs mostram dois comportamentos Unegativo para este centro, onde os estados de carga instáveis são o neutro e o duplamente negativo, e também é observado o deslocamento do oxigênio na direção $\langle 100\rangle$. Devido a este cálculo e os nossos utilizarem o mesmo formalismo do funcional da densidade na aproximação da densidade local (DFT-LDA) e o mesmo tamanho de célula, as discrepâncias podem ser unicamente atribuidas aos diferentes tipos de pseudopotenciais utilizados. 


\subsection{Nitrogênio Substitucional em GaAs}

A impureza de nitrogênio em semicondutores III-V, especificamente em $\mathrm{GaP}$, tem sido estudada desde os anos sessenta devido a seu efeitos nas propriedades ópticas destes materiais, quando forma o sistema de impureza isoeletrônico $\left(N_{P}\right)$. Espectros de absorção deste ${ }^{54}$ sistema mostram varias linhas nos extremos da banda proibida as quais são associadas a estados ligados de excitons na impureza. Estas linhas também foram observadas em outros semicondutores de gap largo. No caso de nitrogênio em $\mathrm{GaAs}$ acreditava-se que os estados de excitons não deveriam se formar devido aos níveis de impureza ser esperados ressonantes na banda de condução. No entanto estes foram observados $^{55}$ o que também confirmaria a existência de níveis de impureza na banda proibida. Recentemente, espectros de fotoluminicência obtidos após a incorporação de nitrogênio, mostram decaimentos radiativos de excitons ligados a pares de nitrogênios em $G a A s .{ }^{56}$ Por outro lado, uma nova linha de pesquisa experimental relacionada com nitrogênio em $\mathrm{GaAs}$ tem despertado o interesse da comunidade científica, envolvendo a fabricação da liga $G a A s_{1-x} N_{x}$. A técnica mais bem sucedida tem sido a de implantação de íons de nitrogênio $\left(N^{+}\right)$de alta energia $(\sim \mathrm{keV})$. Esta nova tecnologia de feixe iônico, possui um alto potencial de implantação de espécies atômicas em materiais hospedeiros, sem estar restrita à condição de equilíbrio térmico, apresentando-se como uma ferramenta versatil na tecnologia do GaAs. Devido às energias envolvidas na implantação iônica, são esperados danos consideráveis na rede e a criação de defeitos envolvendo nitrogênio, ${ }^{57,58}$ principalmente impurezas substitucionais. Cálculos teóricos destes tipos de defeitos em $G a A s$ não tem sido comunicado na literatura, existindo somente em $G a P^{59}$ e na liga $G a P_{x} A s_{1-x},{ }^{60}$ como impureza isoeletrônica.

Nesta seção apresentaremos cálculos teóricos de primeiros princípios da impureza substitucional de nitrogênio em $G a A s$. Estes cálculos foram feitos com o mesmo procedimento utilizado no estudo de oxigênio da seção anterior. A única diferença está na expansão do conjunto base, que agora é considerada equivalente a uma energia de corte de $42 \mathrm{Ry}$, devido ao pseudopotencial do nitrogênio apresentar orbitais de valência menos profundos que o oxigênio, precisando portanto de um número menor de ondas planas no conjunto base. Foram estudados os dois possíveis tipos de impurezas substitucionais, i.e., nitrogênio substituindo um átomo de arsênio $\left(N_{A s}\right)$, que é o sistema isoeletrônico, e nitrogênio substituindo um gálio $\left(N_{G a}\right)$, para diferentes estados de carga. 


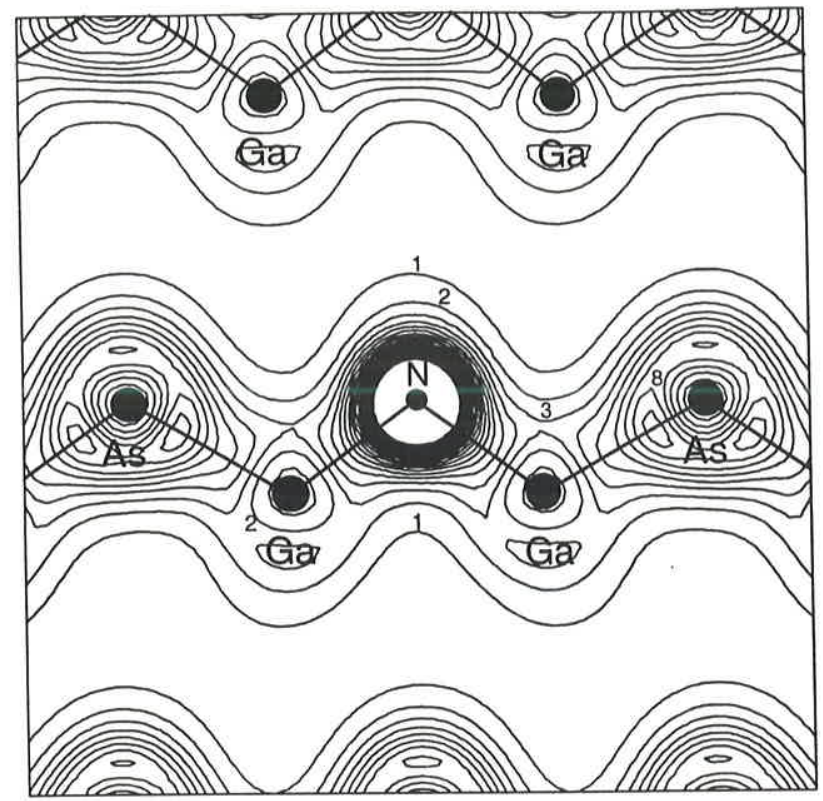

Figura 2.8: Gráfico de contorno da densidade de carga total de valência no plano [110] para a impureza isoeletrônica de nitrogênio substituindo um arsênio em GaAs no estado de carga neutro $\left(N_{A s}^{0}\right)$. Unidades em 10.5 e/ $\Omega$.

\subsubsection{Nitrogênio Substituindo um Arsênio em GaAs}

Nitrogênio substituindo um átomo de arsênio em GaAs no estado de carga neutro, forma uma impureza isovalente, i.e., um átomo do cristal é substituído por outro diferente, pertenecente à mesma coluna da Tabela Periódica. Pelo fato de não existirem elétrons de sobra nas ligações, não são esperados estados ocupados na banda proibida. Nossos resultados para a estrutura eletrônica do defeito $N_{A s}$ em $G a A s$, no estado de carga neutro, mostram um único nível no gap, de simetria $a_{1}$, vazio, localizado a $1.01 \mathrm{eV}$ do topo da banda de valência. O segundo nível vazio é encontrado ressonante no fundo da banda de condução, e sua identificação é difícil devido aos autovalores localizados acima deste apresentarem energias muito próximas, com diferenças em energia dentro do erro associado à dispersão $(\sim 0.1 \mathrm{eV})$. Acreditamos que este segundo nível vazio seja um nível do cristal não degenerado, de simetria $a_{1}$, ao invés de um nível de defeito $t_{2}$, como veremos mais adiante. A geometria de equilíbrio deste centro não apresenta deslocamentos da impureza com respeito ao sítio substitucional, já os quatro gálios primeiros vizinhos se deslocam simetricamente na direção do nitrogênio até alcançar uma distância 

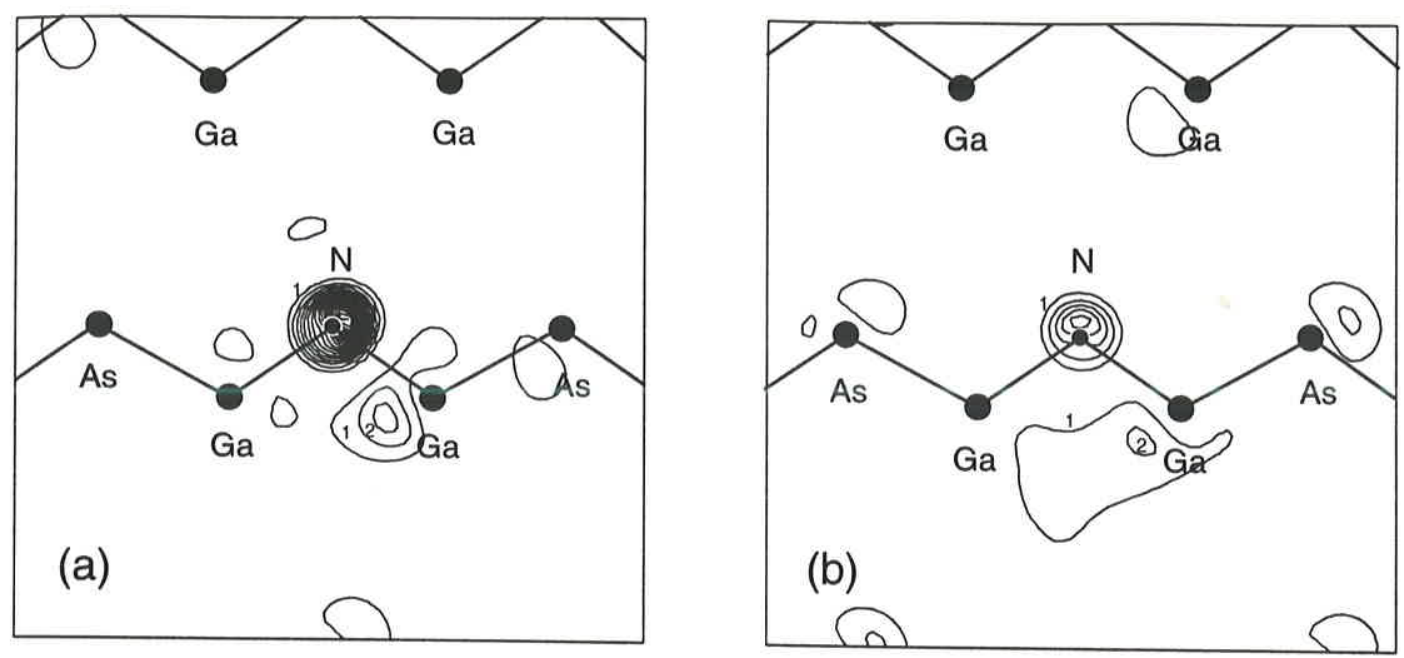

Figura 2.9: Gráficos de contorno da densidade de carga dos dois primeiros níveis vazios do defeito $N_{A s}$ em GaAs, através do plano [110]. (a) Nível $a_{1}$ localizado na banda proibida. (b) Nivel $a_{1}$ localizado ressonante no fundo da banda de condução. Unidades em $0.11 \mathrm{e} / \Omega$.

de ligação de $2.08 \AA$, $14.4 \%$ menor que a distância de ligação $A s$-Ga no cristal puro. Entretanto, a distância de ligação entre os gálios primeiros vizinhos e arsênios segundos vizinhos aumenta 2.8\%. Na Figura 2.8 mostramos o gráfico de contorno da densidade de carga total de valência do defeito $N_{A s}$ em $G a A s$, através do plano [110]. Aqui vemos que o nitrogênio apresenta as mesmas características que o oxigênio quando substitui um arsênio em $G a A s$, i.e., ligações fracas com os primeiros vizinhos e uma grande concentração de carga em volta da impureza. Entretanto notamos que nitrogênio tem uma interação maior com os gálios primeiros vizinhos, produzindo ligações levemente mais fortes devido a este centro ser isoeletrônico, não apresentando orbitais flutuantes. De fato as distâncias de ligação $N$-Ga são aproximadamente $3 \%$ menores que as ligações $O-G a$, para estes dois defeitos no estado de carga neutro.

Na Figura 2.9 mostramos os gráficos de contorno da densidade de carga do nível $a_{1}$ do gap e $a_{1}$ do fundo da banda de condução, ambos vazios, os quais foram disenhados nas mesmas unidades que os níveis $a_{1}$ e $t_{2}$ do defeito $O_{A s}^{0}$ (Figura 2.2). Na Figura 2.9(a) vemos que o nível $a_{1}$ do gap apresenta características similares ao seu equivalente na impureza de oxigênio. O forte caráter atômico, tipo $s$, e a localização da distribuição de carga no defeito confirmam que este é um nível antiligante associado ao orbital atômico $s$ do nitrogênio. O nível ressonante na banda de condução, Figura 2.9(b), também apresenta uma 


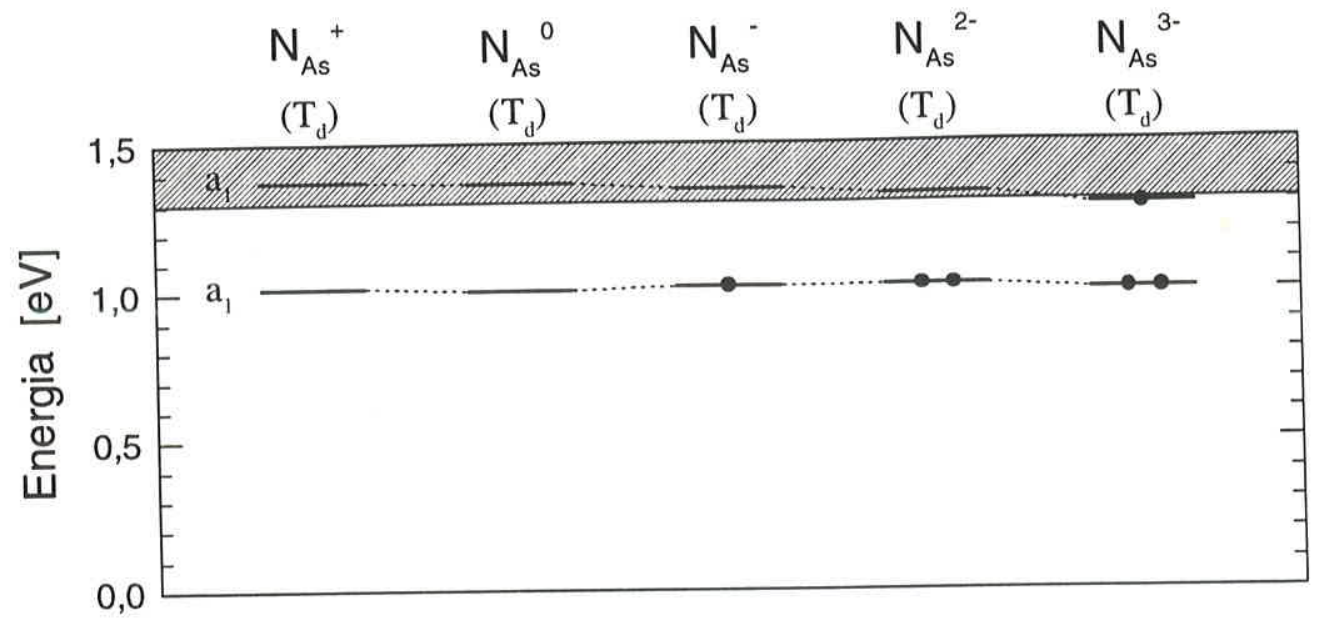

Figura 2.10: Representação esquemática dos autovalores de Kohn-Sham associados aos níveis de defeito do gap para o centro $N_{A s}$ em GaAs, nos cinco estados de carga estudados. Entre parêntesis é indicada a simetria de equilibrio de cada sistema. Os círculos indicam a ocupação dos níveis e a região hachurada representa o fundo da banda de condução.

Tabela 2.2: Geometria de equilíbrio da primeira vizinhança do defeito $N_{A s}$ em GaAs para os cinco estados de carga estudados. $d_{i}$ representa a distância entre os gálios primeiros vizinhos e a impureza $\left(G a_{i}-N\right)$ e $\Delta E_{r}$ a energia de relaxação do sistema.

\begin{tabular}{|c|c|c|c|c|c|}
\hline Defeito & $d_{1}[\AA]$ & $d_{3}[\AA]$ & $d_{3}[\AA]$ & $d_{4}[\AA]$ & $\Delta E_{r}[\mathrm{eV}]$ \\
\hline \hline$N_{A s}^{+}$ & 2.07 & 2.07 & 2.07 & 2.07 & 2.01 \\
\hline$N_{A s}^{0}$ & 2.08 & 2.09 & 2.08 & 2.08 & 1.92 \\
\hline$N_{A s}^{-}$ & 2.07 & 2.07 & 2.07 & 2.07 & 2.10 \\
\hline$N_{A s}^{2-}$ & 2.06 & 2.06 & 2.06 & 2.06 & 2.26 \\
\hline$N_{A s}^{3-}$ & 2.04 & 2.05 & 2.04 & 2.04 & 2.38 \\
\hline
\end{tabular}


distribição de carga com características atômicas tipo $s$ no defeito, mas com uma maior delocalização. As marcadas diferenças com o nível $t_{2}$ do oxigênio, Figura 2.2(b), também localizado ressonante na banda de condução, nos levam a concluir que este nível não é associado ao nitrogênio. Acreditamos que seja um nível não degenerado do cristal, como argumentaremos a seguir.

Calculamos a estrutura eletrônica do centro $N_{A s}$ para outros estados de carga, a representação esquemática dos níveis localizados na banda proibida nas respectivas geometrias de equilíbrio são mostrados na Figura 2.10. Aqui vemos que o nível $a_{1}$ do gap mantém sua posição até ficar completamente ocupado no estado de carga 2-. No estado de carga 3- não é observado nenhum deslocamento considerável da impureza do sítio tetraédrico, logo o último nível ocupado, agora localizado no gap, é não degenerado confirmando nossas suspeitas de se tratar de um nível não degenerado associado ao cristal. Desta forma, o centro $N_{A s}$ em $G a A s$ apresenta simetria $T_{d}$ para todos os estados de carga estudados.

Na Tabela 2.3 é mostrada a geometria de equilíbrio da primeira vizinhança da impureza e a energia de relaxação para o defeito $N_{A s}$ em $G a A s$ nos cinco estados de carga estudados. Aqui notamos que existem diferenças pouco significativas nas distâncias de ligação entre os sistemas com a mesma simetria. O sistema isovalente $\left(N_{A s}^{0}\right)$ apresenta distâncias de ligação levemente maiores e conseqüentemente a menor energia de relaxação entre os diferentes estados de carga, o que também é observado no sistema isovalente da impureza de oxigênio $\left(O_{A s}^{+}\right)$. Isto mostra que a presença de orbitais flutuantes diminui a energia de ligação das impureza. No final desta seção discutiremos mais em detalhe este ponto.

Calculamos a energia de formação do defeito $N_{A s}$ em GaAs para os cinco estados de carga estudados, utilizando o mesmo procedimento discutido na seção anterior (Equação 2.1). Foi simulado um ambiente de crescimento rico em átomos de gálio que é a condição mais favorável para a incorporação deste defeito. O valor máximo ou limite superior para o potencial químico da impureza de nitrogênio foi obtido a partir do cálculo da energia total de nitreto de gálio $(G a N)$, que é o sistema estável que melhor representa a ligação $N-G a$ no sistema de de impureza, e detalhes deste cálculo são mostrados no Apêndice D. Nossos resultados para a energia de formação do defeito $N_{A s}$ em GaAs são mostrados na Figura 2.11. Aqui vemos que este defeito pode existir em quatro diferentes estados de carga ( 0 até 3 -). O estado neutro domina amplamente para $G a A s$ na condição tipo-p e para o semicondutor sem dopagem, mostrando a menor energia de formação. Entretanto os estados negativos aparecem como mais favoráveis em um regime tipo- $n$, como era esperado considerando que 


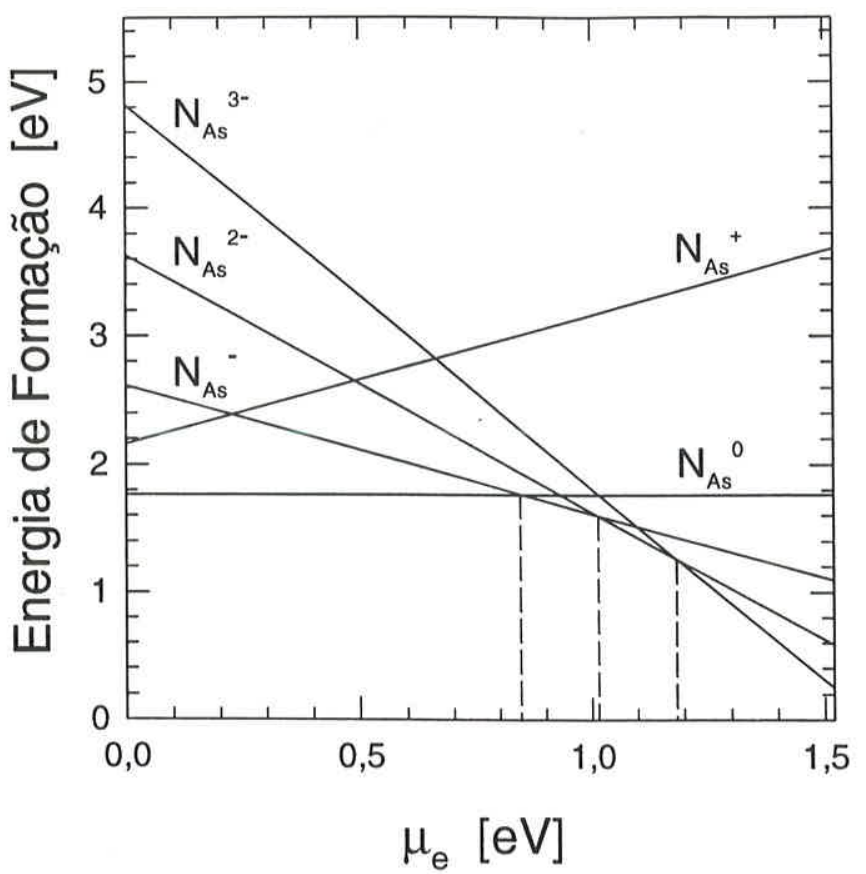

Figura 2.11: Energia de formação do defeito $N_{A s}$ em GaAs como função da posição de nível de Fermi, para um ambiente simulado rico em gálio $\left(\mu_{G a}=\right.$ $\left.\mu_{\text {Ga(bulk) }}\right)$. As linhas tracejadas representam a posição dos estados de transição: $(-/ 0)=0.85 \mathrm{eV},(2-/-)=1.01 \mathrm{eVe}(3-/ 2-)=1.20 \mathrm{eV}$.

o defeito $N_{A s}$ no estado de carga neutro apresenta um nível vazio na parte superior do gap. Estados de carga positivos, que implicariam a remoção de um ou mais eletrons do sistema isovalente, são claramente situações desfavoráveis. Os três estados de transição encontrados no gap se localizam a $E(-/ 0)=E_{v}+$ $0.85 \mathrm{eV}, E(2-/-)=E_{v}+1.01 \mathrm{eV}$ e $E(3-/ 2-)=E_{v}+1.20 \mathrm{eV}$.

Como resumo desta seção podemos dizer que de acordo com nossos resultados a impureza isovalente $N_{A s}$ em $G a A s$ apresenta um nível duplo aceitador na banda proibida, e este nível de defeito não muda sua posição quando são capturados um ou dois elétrons, mantendo-se em aproximadamente $1 \mathrm{eV}$ acima do topo da banda de valência. No estado de carga 3- o último nível ocupado é não degenerado e associado ao cristal, e sua localização é no gap próximo do fundo da banda de condução. Não são observadas distorções da impureza para os diferentes estados de carga estudados, conservando este centro a simetria $T_{d}$. O cálculo da energia de formação mostra que o defeito pode existir em quatro estados de carga (de 0 até 3 -) dando lugar a três estados de transição aceitadores que são acessíveis em um regime tipo- $n$. 


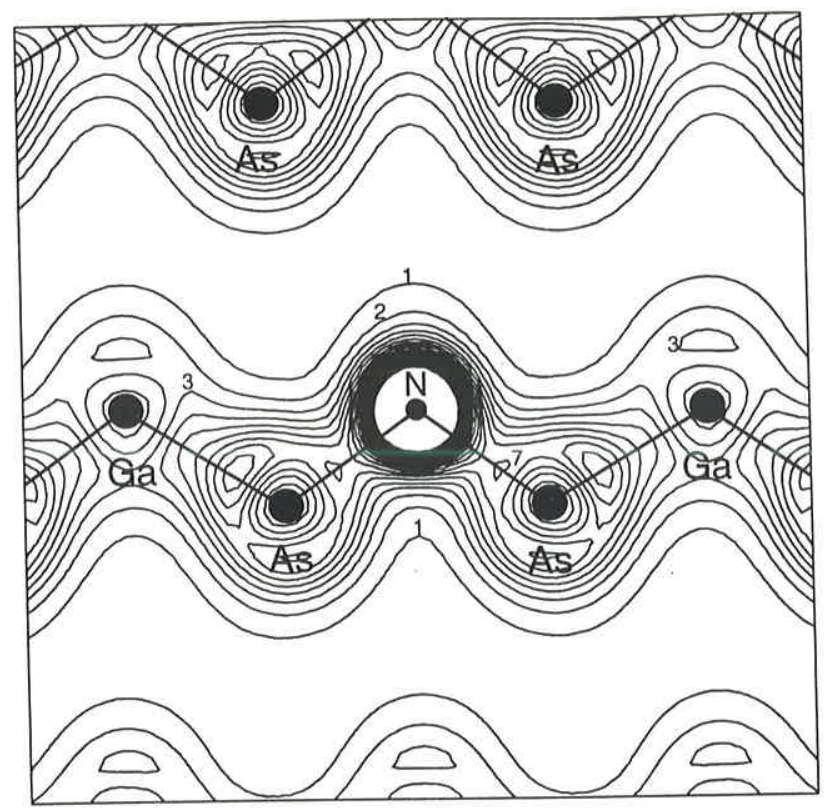

Figura 2.12: Gráfico de contorno da densidade de carga total de valência no plano [110] para o defeito $N_{G a}$ em GaAs no estado de carga neutro. Unidades em $13.5 \mathrm{e} / \Omega$.

\subsubsection{Nitrogênio Substituindo um Gálio em GaAs}

Nossos cálculos da estrutura eletrônica da impureza de nitrogênio substituindo um átomo de gálio em GaAs, no estado de carga neutro, mostra dois níveis de defeito profundos no gap. Um nível $a_{1}$, ocupado com dois elétrons, e um nível $t_{2}$, vazio, localizados respectivamente a 0.3 e $1.1 \mathrm{eV}$ acima do topo da banda de valência, mostrando que este centro é eletricamente inativo. Estes níveis são característicos do defeito e sua ocupação vem do excesso de elétrons introduzidos pelo nitrogênio, que não completam ligações covalentes com os átomos primeiros vizinhos. Não foi observado nenhum deslocamento espontâneo do nitrogênio do seu sítio substitucional. Os quatro arsênios primeiros vizinhos relaxam simetricamente na direção da impureza. A distância de ligação $N$-As na posição de equilíbrio varia levemente para cada arsênio, e a mínima distância de ligação observada é de $2.16 \AA$, aproximadamente $10 \%$ menor que a distância de ligação $G a$-As do cristal puro $(2.43 \AA)$. Entre primeiros e segundos vizinhos, a distância de ligação $A s-G a$ aumenta $2.4 \%$ com respeito do cristal puro. A energia envolvida no processo de relaxação é de $0.81 \mathrm{eV}$. Na Figura 2.12 mostramos o gráfico de contorno da 

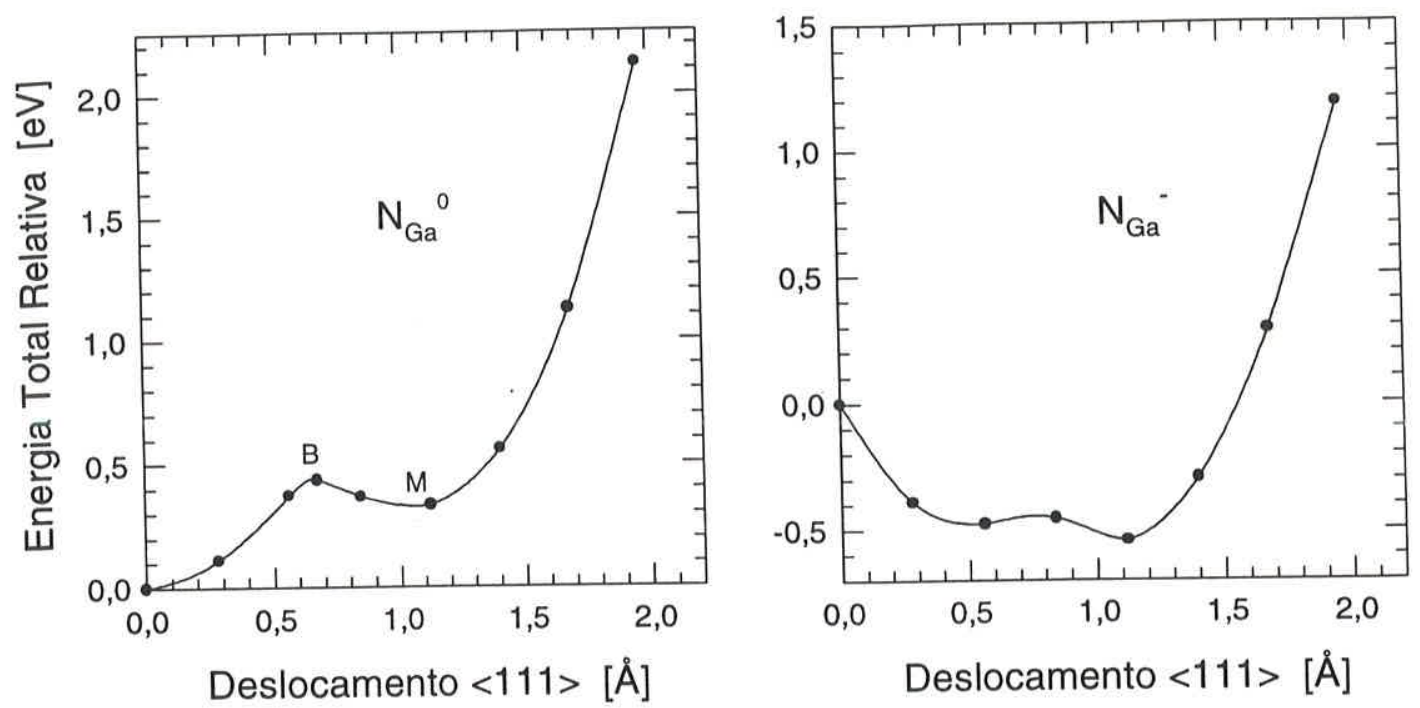

Figura 2.13: Energia total relativa como função do deslocamento do átomo de nitrogênio na direção $\langle 111\rangle$ para os sistemas de impureza $N_{G a}^{0}$ e $N_{G a}^{-}$em GaAs. A energia e deslocamento zero corresponde ao sítio tetraédrico.

densidade de carga de valência para este sistema. Aqui vemos que a ligação do nitrogênio com seus quatro arsênios primeiros vizinhos é forte, onde a impureza atrai para si grande parte da carga das ligações tetraédricas, devido a sua maior eletronegatividade em relação ao arsênio.

Afim de verificarmos possíveis distorções estruturais neste sistema, deslocamos o átomo de nitrogênio na direção $\langle 111\rangle$ nos sistemas neutro e negativo. As curvas de energia total como função do deslocamento para os dois estados de carga considerados são mostrados na Figura 2.13. Aqui observamos um estado de metaestabilidade para o sistema neutro $\left(N_{G a}^{0}\right)$, aproximadamente à $1.05 \AA$ do sítio substitucional (M na Figura 2.13), que também é observado em outras impurezas do tipo antisítio de arsênio em GaAs. ${ }^{61}$ Neste estado metaestável, a energia total do sistema aumenta em $0.32 \mathrm{eV}$, com uma altura da barreira calculada de $0.44 \mathrm{eV}$, e onde seu máximo se localiza a $0.65 \AA$ do sítio substitucional (B na Figura 2.13). Neste cálculo foram utilizados quatro pontos- $k$ de Monkhorst-Pack ${ }^{45}$ afim de se ter uma maior confiabilidade nos resultados. Para o estado de carga negativo $\left(N_{G a}^{-}\right)$observamos um grande deslocamento na direção $\langle 111\rangle$, produzindo uma diminuição da energia total deste sistema da ordem de $0.5 \mathrm{eV}$. A configuração de mínima energia é obtida na mesma posição onde é encontrada a metaestabilidade para o estado neutro. Nesta configuração são observados três níveis no gap: o nível $a_{1}$ caracterítico 


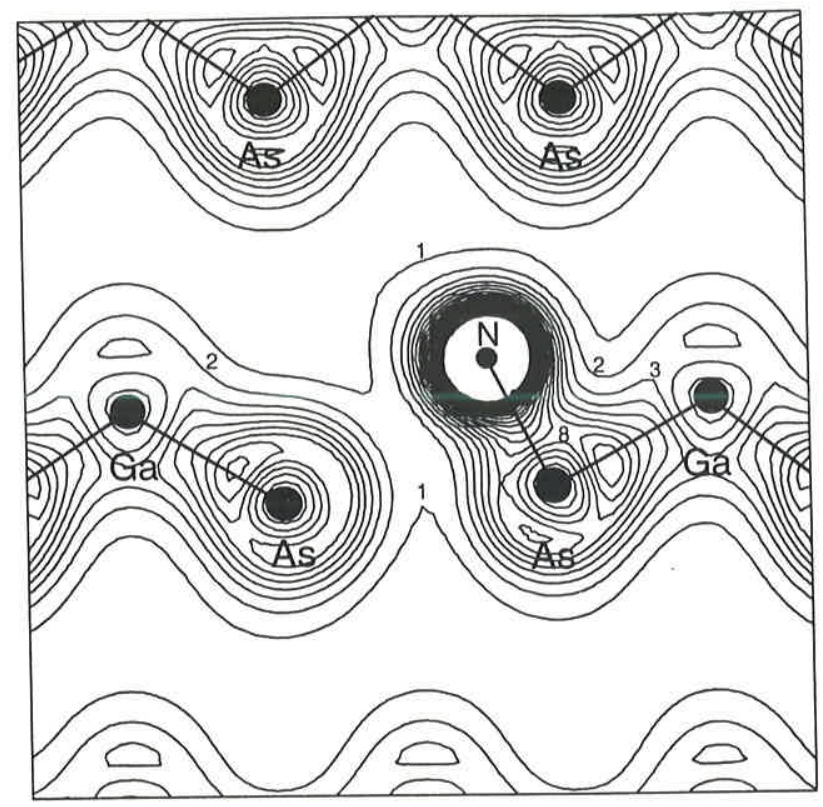

Figura 2.14: Gráfico de contorno da densidade de carga total de valência no plano [110] para o defeito $N_{G a}$ em GaAs no estado de carga neutro, para o estados metaestável. Unidades em $13.5 \mathrm{e} / \Omega$.

do defeito, totalmente ocupado e os dois níveis surgidos do desdobramento do nível $t_{2}$ do defeito, $a_{1}$ ocupado com um elétron e $e$, vazio. Estes três níveis se localizam no gap a $0.23,0.57$ e 1.14 eV acima do topo da banda de valência, respectivamente. O grande deslocamento da impureza com respeito ao sítio substitucional, é atribuído a uma distorção Jahn-Teller a qual é acompanhada por uma diminuição da energia total de $0.58 \mathrm{eV}$ com respeito ao sistema neutro.

Com a finalidade de determinar a natureza do estado metaestável no sistema neutro $N_{G a}^{0}$, calculamos a densidade de carga total de valência através do plano [110] que é mostrada no gráfico de contorno da Figura 2.14. Aqui vemos que nesta posição a impureza se liga fortemente a três gálios, rompendo a ligação com o quarto, i.e., a metaestabilidade é um estado onde a impureza tem coordenação três. A distância de ligação $N$-As nesta configuração é de $1.99 \AA$. Na Figura 2.15 mostramos os gráficos de contorno da densidade de carga dos níveis do gap para a impureza na posição estável (níveis $a_{1}$ ocupado e $t_{2}$ vazio nas Figuras 2.15(a) e 2.15(b), respectivamente) e na posição metaestável (níveis $a_{1}$ ocupado e $a_{1}$ vazio nas Figuras $2.15(\mathrm{c})$ e $2.15(\mathrm{~d})$, respectivamente). Vemos que na posição estável, os níveis no gap apresentam uma distribuição de carga localizada na impureza com característica de orbitais atômicos, própios do 

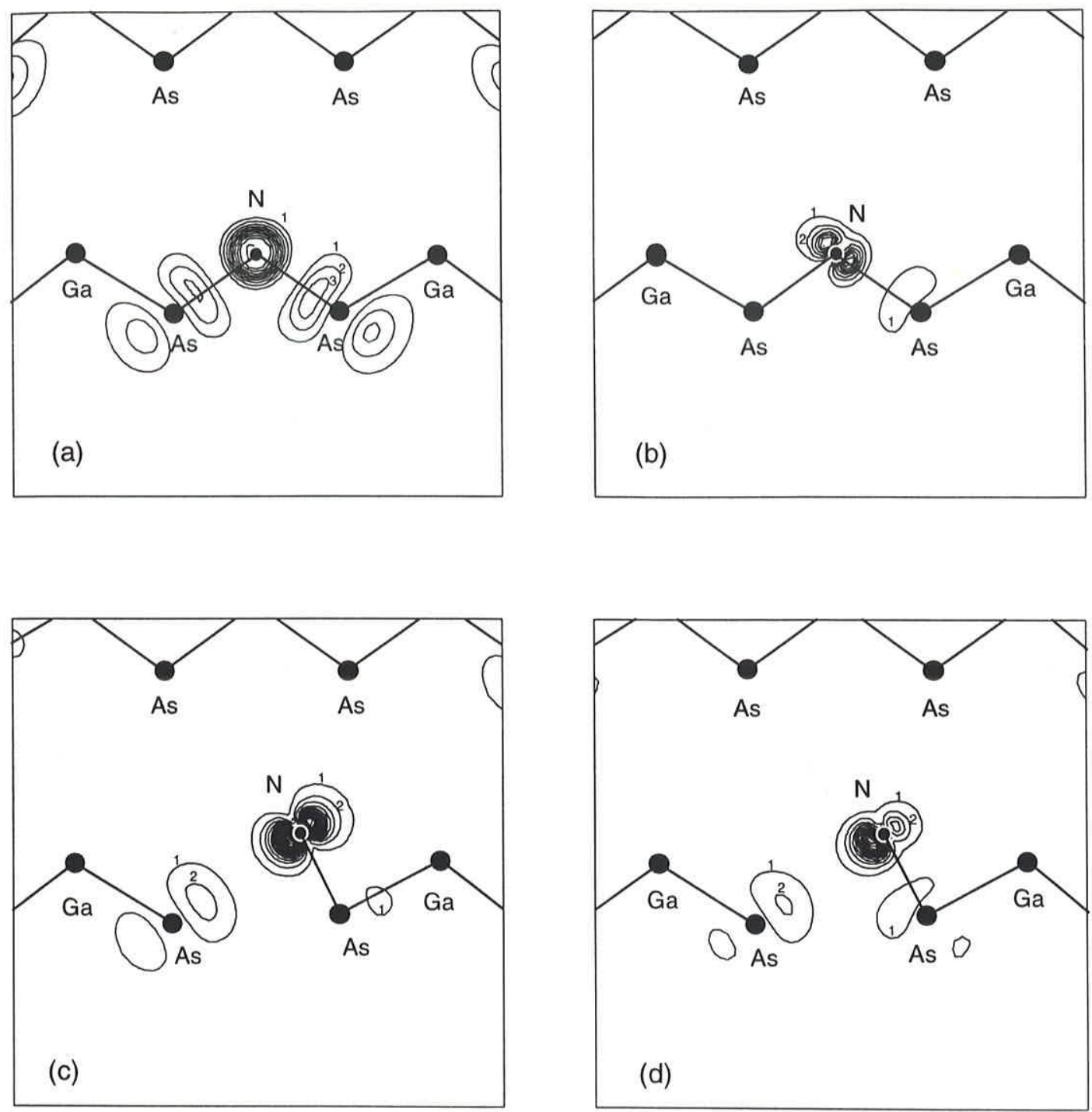

Figura 2.15: Gráfico de contorno da densidade de carga dos níveis do gap através do plano [110] para o defeito $N_{A s}^{0}$ em GaAs. (a) e (b) correspondem às densidades de carga dos niveis $a_{1}$ ocupado com dois elétrons e $t_{2}$ vazio, respectivamente, para a impureza no sítio tetraédrico (estado estável). (c) e (d) correspondem as densidades dos niveis $a_{1}$ ocupado e $a_{1}$ vazio, respectivamente, para a impureza deslocada $1.05 \AA$ na direção $\langle 111\rangle$ (estado metaestável). Unidades em $5.4 \mathrm{e} / \Omega$. 


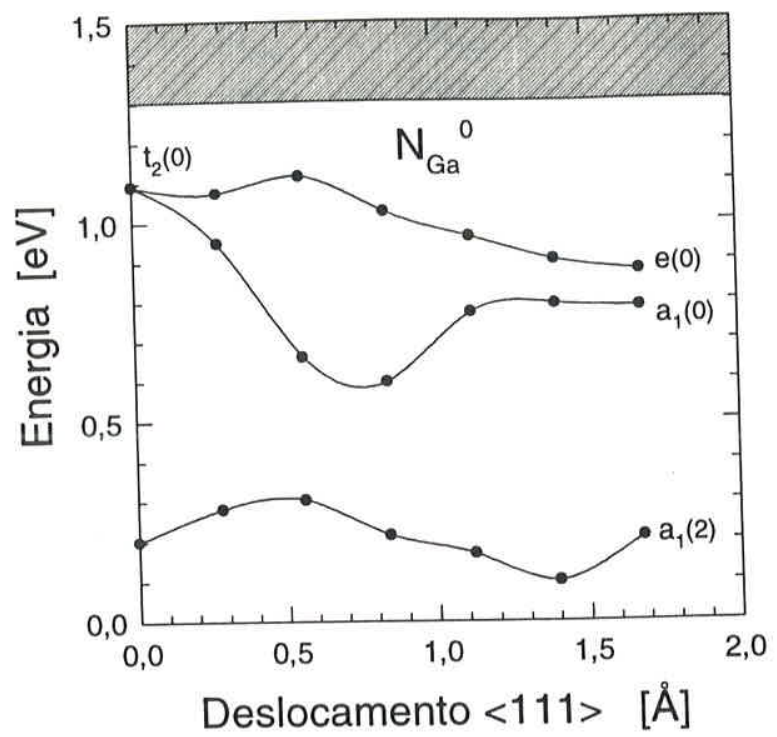

Figura 2.16: Autovalores de Kohn-Sham correspondentes aos niveis no gap, em função do deslocamento do nitrogênio, para o sistema $N_{G a}^{0}$ na direção $\langle 111\rangle$. Entre parêntesis é indicada a ocupação dos niveis. A região hachurada representa a banda de condução.

nitrogênio. Como no sistema $N_{A s}$, nitrogênio prefere atrair carga ao invés de compartilhar-la com sua vizinhança em uma hibridização. O nível $a_{1}$ totalmente ocupado, Figura 2.15(a), mostra um caráter tipo $s$ mais delocalizado que no sistema $N_{A s}$, onde é observada uma carga devida aos orbitais flutuantes, situada nas redondezas dos quatro arsênio primeiros vizinhos. Esta distribuição evidencia uma forte caráter antiligante para a ligações entre nitrogênio e os quatro arsênios primeiros vizinhos. O nível $t_{2}$, vazio, se apresenta localizado e com caráter atômico tipo $p$. No estado metaestável o nível $a_{1}$, totalmente ocupado, Figura 2.15(c), muda seu caráter atômico de tipo $s$ para tipo $p$, ficando orientado na direção normal ao plano formado pelos três gálios com os quais a impureza continua ligada. O nível $a_{1}$, vazio, Figura 2.15(d), mostra uma distribuição de carga similar e a mesma orientação que o nível ocupado. Isto significa que pelo fato das ligações entre a impureza de nitrogênio e os arsênios primeiros vizinhos serem fracas, estas podem se formar a partir de hibridizações $s p^{3}$ ou $s p^{2}$ com igual facilidade, com uma diferença de energia entre elas relativamente baixa $(\sim 0.3 \mathrm{eV})$. Acreditamos que o caráter antiligante observado no orbital $a_{1}$ do gap seja a principal causa da metaestabilidade.

Na Figura 2.16 mostramos a variação dos níveis do gap como função 


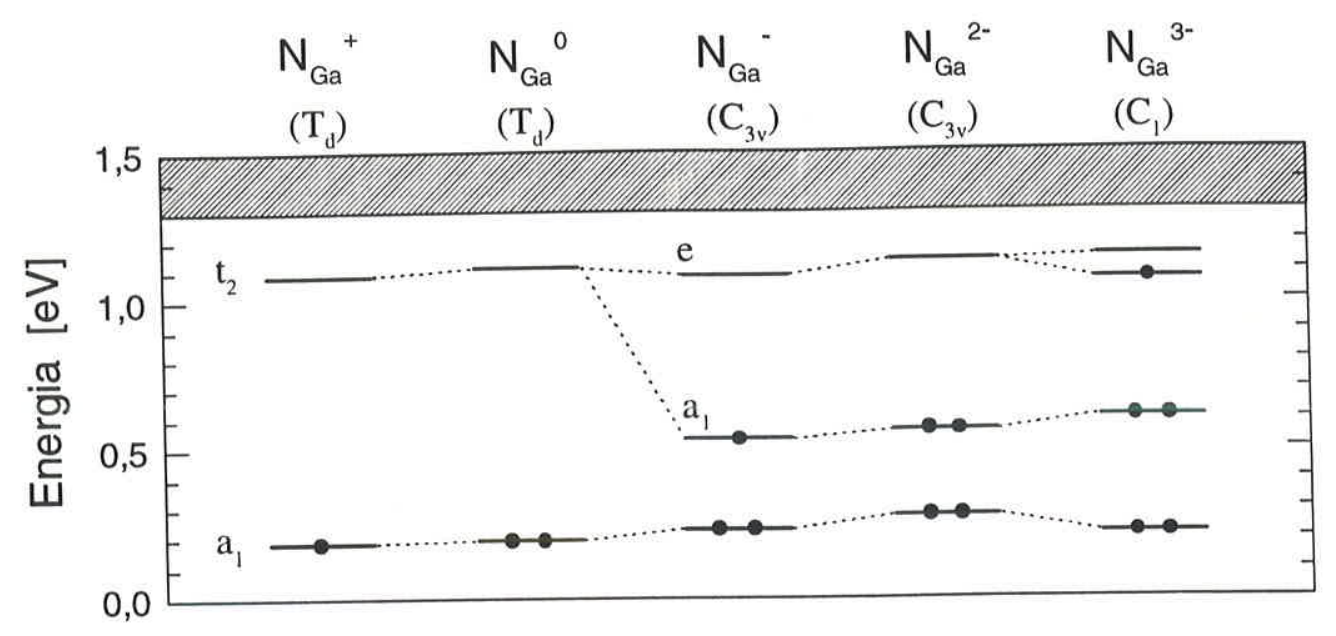

Figura 2.17: Representação esquemática dos autovalores de Kohn-Sham associados aos niveis de defeito no gap para o centro $N_{G a}$ em GaAs, nos cinco estados de carga estudados. Entre parêntesis é indicada a simetria de equilíbrio de cada sistema. Os círculos cheios indicam a ocupação nos níveis e a região hachurada representa o fundo da banda de condução.

do deslocamento da impureza na direção $\langle 111\rangle$ para o sistema $N_{G a}^{0}$ em GaAs. Vemos claramente uma interação entre os níveis de defeito $a_{1}$ totalmente ocupado e $a_{1}$ vazio, originado do desdobramento do nível $t_{2}$ observado no sistema com simetria $T_{d}$. No sítio tetraédrico (deslocamento zero) a diferença em energia entre estes níveis chega a $0.7 \mathrm{eV}$. Conforme a impureza é deslocada na direção $\langle 111\rangle$ (simetria $C_{3 v}$ ), o nível $a_{1}$ vazio se aproxima do nível ocupado, diminuindo a diferença em energia entre eles até um mínimo de $0.3 \mathrm{eV}$, que ocorre aproximadamente no topo da barreira (B na Figura 2.13). No sítio metaestável, a $1.05 \AA$ do sítio tetraédrico, os níveis de impureza, $a_{1}(2), a_{1}(0)$ e $e(0)$, se localizam aproximadamente a $0.2,0.8$ e $1.0 \mathrm{eV}$ acima do topo da banda de valência, respectivamente.

Nossos resultados para o defeito $N_{G a}$ no estados de carga 2- mostram uma geometria similar à observada no sistema negativo, e a configuração de mínima energia é obtida com a impureza localizada a $0.8 \AA$ do sítio tetraédrico na direção $\langle 111\rangle$. Os níveis de defeito no gap apresentam aproximadamente as mesmas posições que no sistema negativo, a única diferença está na ocupação pois neste último caso os dois níveis $a_{1}$ se encontram totalmente ocupados. No estado de carga 3- o nível $e$ é ocupado e a impureza sofre uma distorção Jahn-Teller, deslocando-se $0.15 \AA$ na direção $\langle 010\rangle$ a partir da posição de equilíbrio na qual se encontrava no estado de carga 2-. A estrutura eletrônica 
Tabela 2.3: Geometria de equilíbrio da primeira vizinhança do defeito $N_{G a} \mathrm{em}$ GaAs para os cinco estados de carga estudados. $d_{i}$ representa a distância entre os quatro arsênios primeiros vizinhos e a impureza $\left(A s_{i}-N\right)$ e $\Delta E_{r}$ a energia de relaxação do sistema.

\begin{tabular}{|c|c|c|c|c|c|}
\hline Defeito & $d_{1}[\AA]$ & $d_{2}[\AA]$ & $d_{3}[\AA]$ & $d_{4}[\AA]$ & $\Delta E_{r}[\mathrm{eV}]$ \\
\hline \hline$N_{G a}^{+}$ & 2.18 & 2.16 & 2.14 & 2.18 & 0.94 \\
\hline$N_{G a}^{0}$ & 2.21 & 2.17 & 2.16 & 2.21 & 0.81 \\
\hline$N_{G a}^{-}$ & 2.01 & 3.20 & 2.00 & 2.01 & 1.39 \\
\hline$N_{G a}^{2-}$ & 2.02 & 3.00 & 2.04 & 2.02 & 2.16 \\
\hline$N_{G a}^{3-}$ & 1.97 & 3.06 & 2.06 & 1.96 & 2.38 \\
\hline
\end{tabular}

para o sistema $N_{G a}^{3-}$ mostra quatro níveis no gap: os dois níveis $a_{1}$ totalmente ocupados e dois níveis não degenerados produto do desdobramento do nível $e$ devido à redução da simetria pontual do sistema, que nesta configuração não apresenta elementos de simetria (grupo de simetria $C_{1}$ ). Estes níveis de defeito se localizam a $0.22,0.61,1.07$ e $1.15 \mathrm{eV}$ acima do topo da banda de valência respectivamente.

Na Figura 2.17 mostramos esquematicamente a evolução dos níveis de impureza da banda proibida para o defeito $N_{G a}$ em GaAs, na medida que são adicionados elétrons ao sistema. Aqui notamos que para todos os estados de carga estudados, os níveis de defeito se localizam no gap. Não são observadas grandes variações destes níveis para sistemas com mesma simetria, e as variações importantes ocorrem sempre acompanhadas por quebras de simetria as quais são atribuidas à distorções Jahn-Teller. Na Tabela 2.3 mostramos as distâncias de ligação entre o nitrogênio e seus quatro gálios primeiros vizinhos na configuração de mínima energia e a respectiva energia de relaxação do sistema para os cinco estados de carga estudados.

Calculamos também a energia de formação do defeito $N_{G a}$ em $G a A s$ nos cinco estados de carga, para um ambiente simulado rico em arsênio. Neste caso, o limite superior do potencial químico da impureza foi obtido a partir do cálculo da energia total da molécula de nitrogênio $\left(N_{2}\right)$. Consideramos este sistema devido à ligação $N-N$ na molécula de nitrogênio ser mais estável que qualquer outro sistema que apresente uma ligação isovalente com nitrogênio. Nossos resultados para a energia de formação são mostrados na Figura 2.18. Nesta figura vemos que o defeito $N_{G a}$ em GaAs pode existir em quatro diferentes estados de carga (de 0 até 3 -), apresentando somente níveis aceitadores 


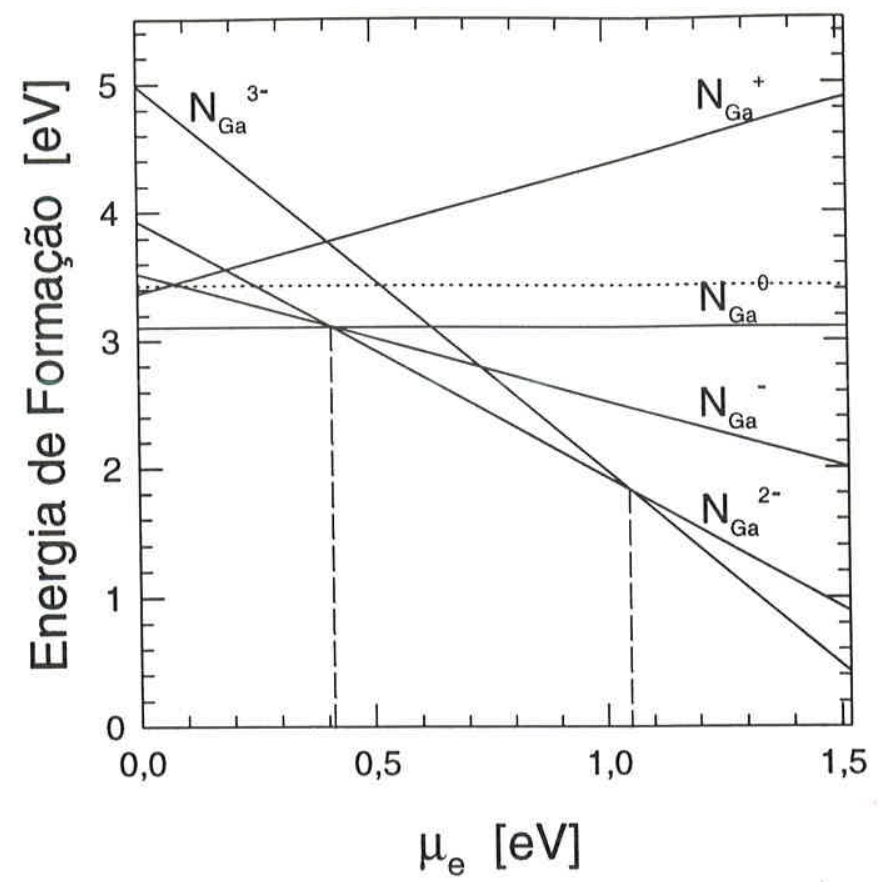

Figura 2.18: Energia de formação do defeito $N_{G a}$ em GaAs como função da posição de nivel de Fermi, para um ambiente simulado rico em arsênio $\left(\mu_{A s}=\right.$ $\left.\mu_{A s(b u l k)}\right)$. A linha pontilhada representa a energia de formação para o defeito $N_{G a}^{0}$, na estado metaestável, e as linhas tracejadas a posição dos estados de transição: $(2-/ 0)=0.41 \mathrm{eV},(3-/ 2-)=1.05 \mathrm{eV}$.

no gap. Estes encontram-se localizados a: $E(-/ 0)=E_{v}+0.42 \mathrm{eV}, E(2-/-)$ $=E_{v}+0.41 \mathrm{eV}$ e $E(3-/ 2-)=E_{v}+1.05 \mathrm{eV}$. Como o aceitador duplo (2/-) é encontrado acima do aceitador simples (-/0), este centro apresenta um comportamento U-negativo $(|\mathrm{U}|=0.01 \mathrm{eV})$, dando lugar a uma transição envolvendo dois elétrons (2-/0). Desta forma, o defeito $N_{G a}$ no estado de carga negativo é instável. Notamos ainda na Figura 2.18 que para $G a A s$ tipo-p, é mais provável encontrar o defeito no estado de carga neutro, enquanto que para GaAs tipo-n aparece mais favorável o estado de carga triplamente negativo. Para o semicondutor sem dopagem intríseca o estado duplamente negativo domina amplamente. Se consideramos o defeito $N_{G a}^{0}$ no estado metaestável, o que implica um aumento na energia de formação de $0.32 \mathrm{eV}$ acima do estado estável (linha pontilhada na Figura 2.18), encontramos uma configuração totalmente diferente para os estados eletrônicos do defeito no regime tipo- $p$. Nesta situação o defeito $N_{G a}$ pode existir em cinco estados de carga estáveis (de 1+ até $3-$ ), sendo os novos estados doador e aceitador simples, encontrados 
a $E(0 /+)=E_{v}+0.06 \mathrm{eV}$ e $E(-/ 0)=E_{v}+0.10 \mathrm{eV}$, respectivamente. Assim quando o defeito $N_{G a}^{0}$ em $G a A s$ tipo- $p$ ocupa a posição de metaestabilidade estrutural, ele pode ser encontrado nos estados de carga positivo, neutro e negativo.

Como resumo desta seção podemos dizer que a impureza de nitrogênio substituindo um gálio em $G a A s$, no estado de carga neutro, apresenta uma metaestabilidade estrutural de forma similar à observada em outras impurezas da coluna V da Tabela Periódica em GaAs. No estado estável o nitrogênio fica fortemente ligado a seus quatro arsênios primeiros vizinhos, enquanto que no estado metaestável uma das ligações é rompida deixando a impureza com coordenação três, equidistante de seu três arsênios primeiros vizinhos. A estrutura eletrônica do defeito $N_{G a}^{0}$ em GaAs mostra dois níveis de defeito no gap, de simetria $a_{1}$ e $t_{2}$. Ao adicionar um elétron ao sistema, é observado um grande deslocamento da impureza na direção $\langle 111\rangle$, atribuida a uma distorção Jahn-Teller, provocando o desdobramento do nível tridegenerado $\left(t_{2}\right)$. Uma segunda distorção Jahn-Teller de menor intensidade é observada no estado de carga 3-, onde neste caso é desdobrado um nível bidegenerado $(e)$. Os cálculos da energia de formação mostram que este defeito é estável em três estados de carga (0,2- e 3-), apresentando um comportamento U-negativo e dois níveis aceitadores no gap, (-/0) e (2-/-). Se o defeito no estado de carga neutro é colocado na posição de metaestabilidade estrutural, o sistema apresenta cinco estados de carga estáveis (de 1+ até 3-) e portanto um nível doador e três aceitadores no gap.

Na Figura 2.19 comparamos os resultados do cálculo da energia de formação para os dois sistemas da impureza substitucional de nitrogênio em $G a A s$, nos ambientes simulados rico em átomos de arsênio e gálio. Aqui podemos ver que em ambos os ambientes e na condição tipo- $p$, a impureza $N_{A s}^{0}$ mostra a menor energia de formação, aparecendo como o defeito dominante mesmo em um ambiente rico em arsênio onde sua ocorrência é menos favorável. No ambiente rico em arsênio e na condição tipo-n, os sistemas $N_{G a}^{3-}$ e $N_{A s}^{3-}$ mostram valores da energias de formação próximos $(\sim 0.2 \mathrm{eV})$ evidenciando um processo de competição entre eles, onde a impureza $N_{G a}^{3-}$ aparece como mais favorável. Para uma condição sem dopagem intrinseca, i.e., com o nível de Fermi no meio do gap, os sistemas $N_{G a}^{2-}, N_{A s}^{0}$ e $N_{A s}^{-}$apresentam aproximadamente a mesma energia de formação, significando que a impureza de nitrogênio nestas condições pode ser encontrada com igual probabilidade. Para um ambiente rico em átomos de gálio vemos que o sistema $N_{A s}$ apresenta a menor energia de formação em todo o intervalo de energia do gap, mostrando que é a impureza dominante para todos os estados de carga estudados. Na 

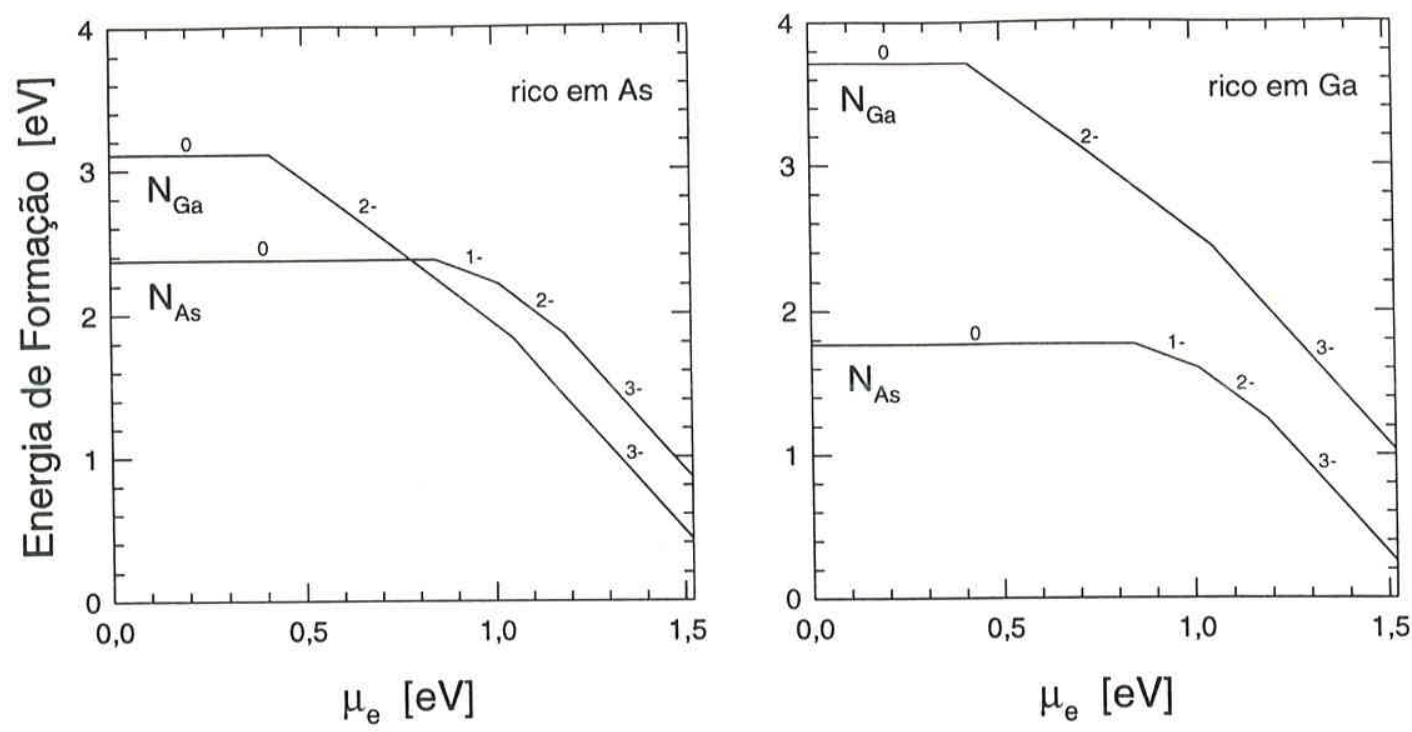

Figura 2.19: Energia de formação das impurezas $N_{A s}$ e $N_{G a}$ em GaAs para ambientes simulados rico em átomos de arsênio e gálio. A inclinação das retas representa o estado de carga dos sistemas.

condição tipo- $p$, a impureza $N_{G a}^{0}$ apresenta uma energia de formação da ordem de $4 \mathrm{eV}$, dois $\mathrm{eV}$ a mais que a impureza $N_{A s}^{0}$. Isto mostra que em equilíbrio termodinâmico este sistema é pouco provável de se formar. Na condição tipo$n$, a impureza $N_{G a}^{3-}$ apresenta energias de formação da ordem daquelas obtidas para $N_{A s}$ nos estados 2- e 3-, fazendo destas impurezas processos competitivos.

A energia de ligação de uma impureza susbtitucional no cristal pode ser calculada considerando-se uma reação entre esta impureza em um sítio intersticial e a vacância do sítio substitucional, ambas no estado de carga neutro. Tomando como exemplo a impureza substitucional de oxigênio, esta seria: $O_{i}^{0}+V_{A s}^{0} \rightarrow O_{A s}^{0}$. Desta forma a energia de ligação é definida como a energia necessária para remover a impureza do sítio substitucional deixando em seu lugar uma vacância, sendo a posição final da impureza escolhida como sendo o sítio intersticial $\mathrm{T}$ onde a interação com o cristal é minimizada. Desta forma podemos calcular a energia de ligação $E_{b}$ do oxigênio utilizando a seguinte relação:

$$
-E_{b}=E_{t}\left(O_{A s}\right)-E_{t}\left(V_{A s}\right)+E_{t}\left(O_{T}\right)-E_{t}(G a A s),
$$

onde $E_{t}$ é a energia total dos sistemas neutros: impurezas de oxigênio substitucional $\left(O_{A s}\right)$, vacância de arsênio $\left(V_{A s}\right)$, impureza intersticial de oxigênio 
localizada no sítio $\mathrm{T}\left(O_{T}\right)$ e cristal de GaAs. Estes sistemas foram calculados considerando os mesmos parâmetros para os psedopotenciais, critérios de convergência e tamanho de célula. $\mathrm{O}$ sinal de $E_{b}$ é escolhido de modo que o valor positivo da energia de ligação indica uma configuração com a impureza ligada. Nossos resultados para as três impurezas substitucionais consideradas são: $E_{b}\left(O_{A s}\right)=6.01 \mathrm{eV}, E_{b}\left(N_{A s}\right)=7.81 \mathrm{eV}, E_{b}\left(N_{G a}\right)=4.50 \mathrm{eV}$. Notamos que a impureza isoeletrônica de nitrogênio $\left(N_{A s}\right)$ apresenta a maior energia de ligação apesar do oxigênio ser a impureza mais eletronegativa. Nitrogênio com dois orbitais flutuantes $\left(N_{G a}\right)$ mostra uma energia de ligação da ordem de $3.3 \mathrm{eV}$ menor que $N_{A s}$. No caso da impureza de oxigênio $\left(O_{A s}\right)$ que apresenta um orbital flutuante, a energia de ligação é $1.8 \mathrm{eV}$ menor que $N_{A s}$. Concluimos que no caso destas impurezas, a presença de orbitais flutuantes diminui a energia de ligação de impureza em aproximadamente $1.6 \mathrm{eV}$. 


\section{Capítulo 3}

\section{Impurezas Complexas $O-H$ e $N-H$ em $G a A s$}

O hidrogênio é uma das impurezas residuais mais comuns observadas em compostos III-V, interagindo das mais diversas formas com os átomos hospedeiros ou defeitos nativos e impurezas presentes no cristal. Os principais processos envolvendo o átomo de hidrogênio são a passivação de níveis rasos e profundos eletricamente ativos e a ativação de centros isoeletrônicos, normalmente não ativos. O estudo da passivação de centros de impureza teve seu inicio com os trabalhos de Sah et al..$^{62}$ e Pankove et al. ${ }^{63}$ onde foi demostrado que o hidrogênio passiva níveis aceitadores, introduzidos pela impureza de boro em silício. Posteriormente um grande número de experiências confirmaram a passivação de outros níveis aceitadores e doadores rasos em silício e praticamente todos os dopantes rasos em compostos III-V ${ }^{64}$ Aplicações tecnológicas exploram a passivação para melhorar materiais e dispositivos, mas ainda mais importante que a passivação, é a introdução não intencional de hidrogênio durante o crescimento e procesamento de semicondutores, pois átomos de hidrogênio podem migrar nos dispositivos em operação alterando suas características. Este comportamento tem motivado consideráveis esforços na compreenção da formação e propriedades relacionadas com dopantes e átomos de hidrogênio. O estudo da estrutura eletrônica e propriedades de complexos envolvendo hidrogênio e impurezas profundas em compostos III-V são escassos, e recentemente estudos teóricos tem mostrado a passivação do defeito $E L 2^{65}$ e da impureza substitucional de carbono em GaAs. ${ }^{66-68}$

Neste capítulo apresentaremos cálculos de energia total dos sistemas complexos: $O_{A s^{-}}-H, N_{A s}-H$ e $N_{G a}-H$ em $G a A s$, com hidrogênio incorporado intersticialmente. Nosso objetivo é estudar a estrutura eletrônica destes cen- 


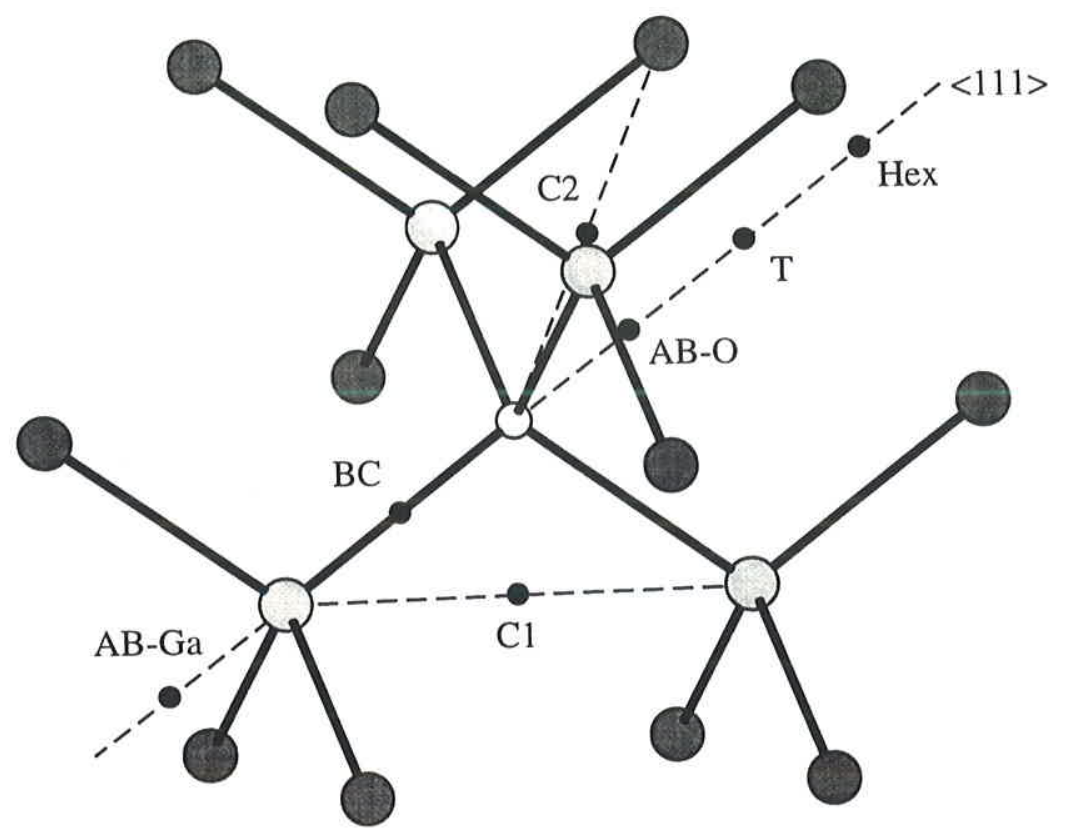

$\mathrm{H}$
$\mathrm{O}$

Figura 3.1: Representação esquemática dos sítios intersticiais do átomo de hidrogênio usados como configurações inicias no estudo do complexo $O_{A s}-H$ em GaAs.

tros, especificamente a passivação dos centros eletricamente ativos e a ativação dos centros inativos obtendo a configuração de mínima energia dos complexos. Os cálculos foram realizados em uma célula bcc de 32 átomos e utilizando os mesmos parâmetros de pseudopotencial e critérios de convergência de forças que nos cálculos das impurezas substitucionais, estudadas no capítulo anterior. Para determinar a configuração atômica de mínima energia, o átomo de hidrogênio foi colocado em sete sítios intersticiais a partir dos quais as impurezas e duas camadas de átomos hospedeiros foram permitidos de relaxar, estas posições são representadas esquematicamente na Figura 3.1. Nesta figura definimos o sítio centrado na ligação $(\mathrm{BC})$, sítio antiligante respeito à impureza de oxigênio $(\mathrm{AB}-\mathrm{O})$, o sítio antiligante respeito a um gálio primeiro vizinho (AB-Ga), o sítio tetraédrico ( $\mathrm{T})$, o sítio hexagonal (Hex), o sítio centrado na linha reta que une dois gálios primeiros vizinhos $(\mathrm{C} 1)$ e o sítio centrado na linha reta que une um arsênio segundo vizinho e o oxigênio $(\mathrm{C} 2)$. 


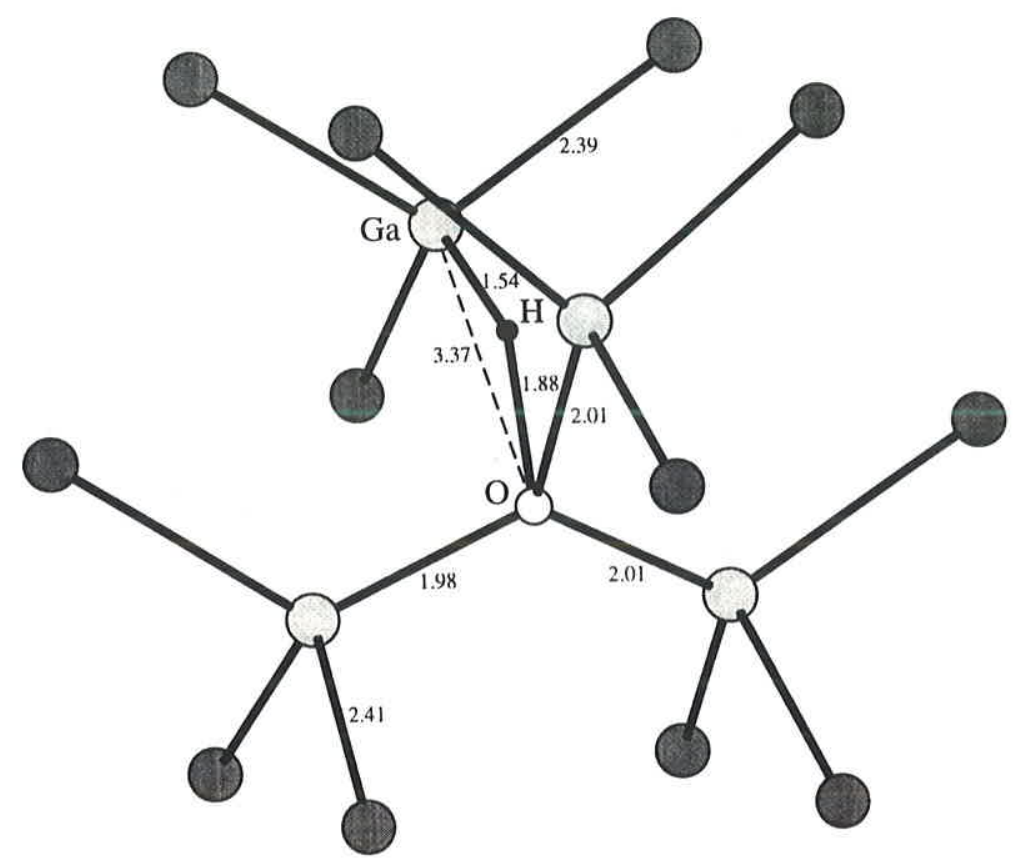

Figura 3.2: Configuração atômica de mínima energia obtida para o complexo $O_{A s}-H$ em GaAs. Os números representam as distâncias de ligação entre os átomos respectivos, em ångstroms.

\subsection{Complexo $O_{A s}-H$ em GaAs}

Nossos resultados mostram que a configuração que apresenta a menor energia total entre os diferentes sítios estudados, tem o átomo de hidrogênio localizado próximo do centro de uma ligação $O-G a$ levemente distorcida, formando um ângulo $O-H$-Ga de aproximadamente $160^{\circ}$. Esta configuração foi obtida a partir do sítio intersticial C2 da Figura 3.1. A geometria atômica de equilíbrio para os átomos que são permitidos de relaxar, são representadas na Figura 3.2, onde também são mostradas as distâncias de ligação mais relevantes. A configuração com a segunda menor energia, $0.1 \mathrm{eV}$ a mais, foi obtida a partir do sítio C1. Nesta, o hidrogênio aparece mais afastado do oxigênio mas mantendo as mesmas características que no sistema de mínima energia. Na Figura 3.3 mostramos os gráficos de contorno da densidade de carga total de valência para estas duas configurações. Na Figura 3.3(a) é mostrado a densidade de carga total através do plano [011] para a configuração de mínima energia (C2). Aqui vemos que o hidrogênio se liga fracamente ao seus vizinhos mantendo uma certa preferência pelo gálio, enquanto que oxigênio conserva 

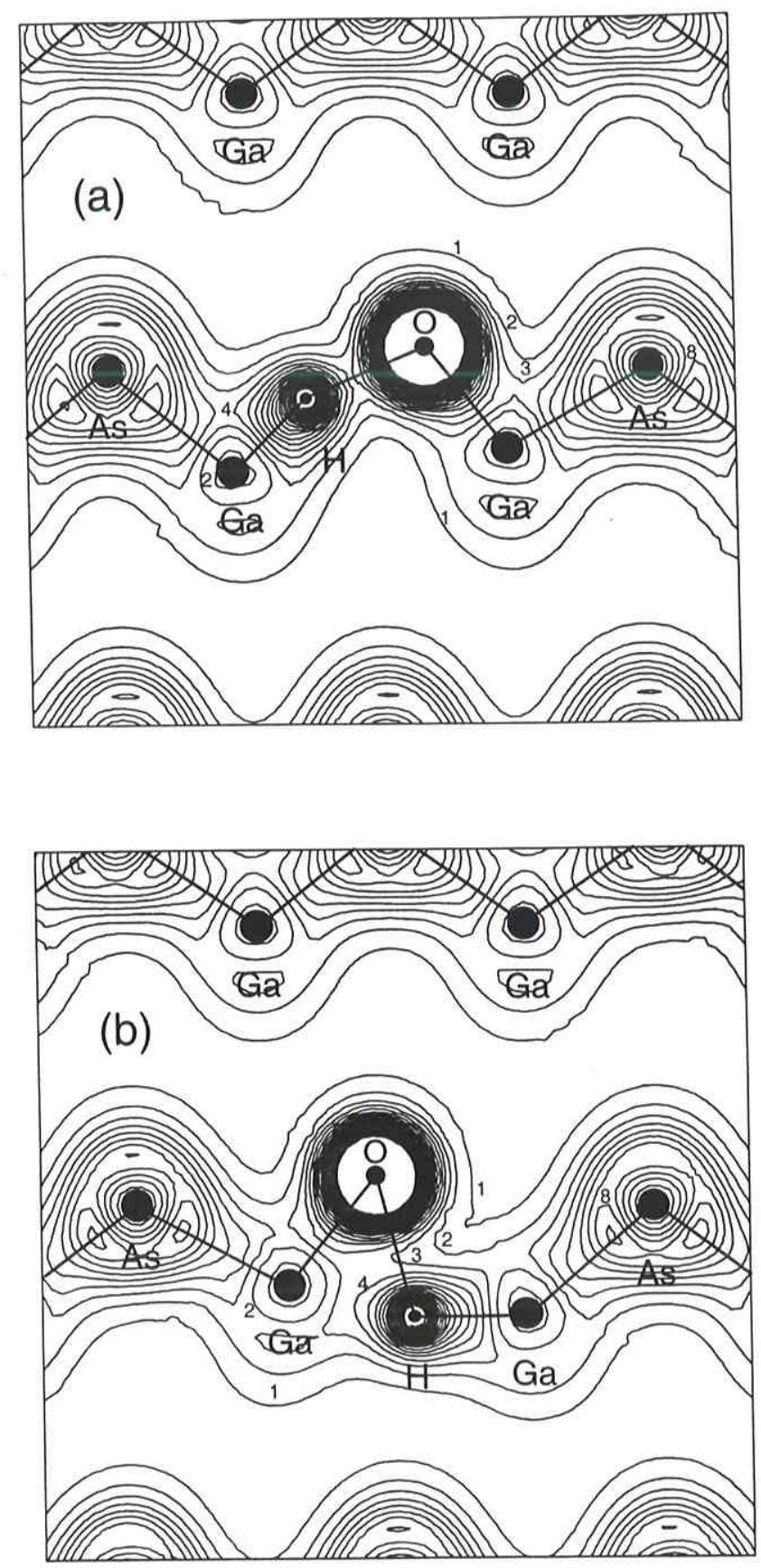

Figura 3.3: Gráficos de contorno da densidade de carga total de valência para as duas configurações de menor energia do complexo $O_{A s}-H$ em GaAs. (a) Configuração obtida a partir do sítio $\mathrm{C} 2$, no plano [011]. (b) Configuração obtida a partir do sítio $\mathrm{C} 1$, no plano [110]. Unidades em $12.5 \mathrm{e} / \Omega$. 
sua característica atômica. Estes átomos se deslocam com respeito a seus sítios regulares em direções opostas com a introdução do hidrogênio, oxigênio se afasta $0.76 \AA$ ficando aproximadamente no mesmo plano formado pelos três gálios nos quais continua ligado, enquanto que o gálio ligado ao hidrogênio se afasta $0.38 \AA$ na direção de seus arsênios primeiros vizinhos. As distâncias de ligação $O-H$ e $H$-Ga são calculadas em 1.88 e $1.54 \AA$ respectivamente. Na Figura 3.3(b) mostramos a densidade de carga total através do plano [110] da configuração que apresenta a segunda menor energia total (C1). Aqui vemos que o hidrogênio fica mais afastado do oxigênio, não chegando à posição centrada na ligação, devido à interação com os dois gálios, e ocupando uma posição de mínimo local. As distâncias de ligação $O-H$ e $H$-Ga são encontradas em 2.19 e $1.67 \AA$ respectivamente, enquanto que o ângulo $O$ - $H$-Ga é de aproximadamente $101^{\circ}$. Uma outra configuração similar à C1 foi encontrada a partir do sítio T. Esta apresenta uma energia total de $0.17 \mathrm{eV}$ a mais que a configuração de mínima energia (C2), e as distâncias de ligação $O-H$ e $H$-Ga são encontradas em 2.21 e $1.62 \AA$ respectivamente, com um ângulo $O$ - $H$ - $G a$ de $87^{\circ}$.

Na Figura 3.4 mostramos os gráficos de contorno da densidade de carga total de valência para as configurações obtidas a partir dos sítios intersticiais BC e AB-O do hidrogênio. A Figura 3.4(a) mostra a configuração de equilíbrio obtida a partir do sítio BC. Aqui observamos a formação de um dimero $O H$, que se liga fracamente a rede, cuja distância de ligação é calculada em $1.04 \AA$, levemente maior que a distância de ligação $O-H$ na molécula de água, calculada em $0.97 \AA$ (Apêndice C). Esta configuração apresenta uma energia total de $0.36 \mathrm{eV}$ maior que a da configuração de mínima energia. Notamos que oxigênio se desloca $0.54 \AA$ do sítio substitucional na direção $\langle 111\rangle$ conjuntamente com hidrogênio, que por sua vez se desloca $0.7 \AA$ do sítio $\mathrm{BC}$, enquanto que o gálio não sofre nenhum deslocamento importante. Na Figura 3.4(b) mostramos a configuração de equilíbrio obtida a partir do sítio AB-O do hidrogênio. Aqui vemos que, da mesma forma que no sítio BC, o hidrogênio e o oxigênio formam um dimero. A distância de ligação $O-H$ é calculada em $1.05 \AA$ e não são observados grandes deslocamentos atômicos. A energia total desta configuração é de 1.47 eV maior que a configuração de mínima energia, logo bem maior que no sistema $\mathrm{BC}$. O aumento na energia total para a configuração AB- $O$ pode ser explicada pela ligação a mais que apresenta o átomo de oxigênio, i.e., os quatro gálios primeiros vizinhos mais o hidrogênio. Observamos que a formação do dimero $O H$ depende do sítio onde o hidrogênio é inicialmente deixado e da proximidade com o oxigênio. Nas duas configurações onde é observado (AB-O e BC) a distância inicial $O-H$ é 

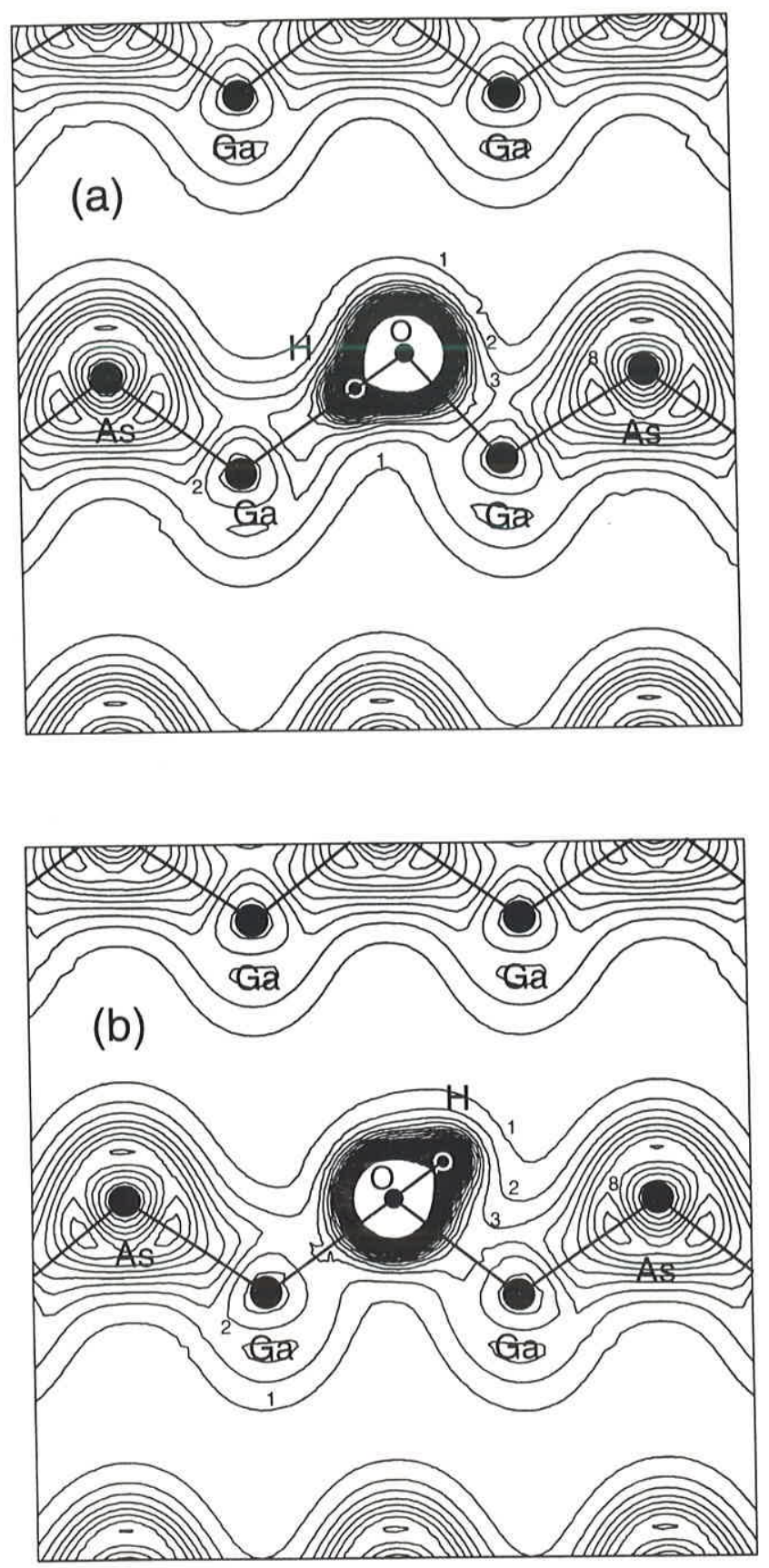

Figura 3.4: Gráficos de contorno da densidade de carga total de valência, através do plano [110], para duas configurações de equilíbrio do complexo $O_{A s^{-}}$H em GaAs. (a) Configuração obtida a partir do sítio BC. (b) Configuração obtida a partir do sitio AB-O. Unidades em 12.5 e/ $\Omega$. 
de $1.2 \AA$ e, para distâncias maiores que este valor, o dimero não se forma. Logo como a configuração que apresenta a mínima energia é obtida a partir do sítio $\mathrm{C} 2$, onde a distância $O-H$ é de aproximadamente $2 \AA$, a formação do dimero originada a partir de uma interação entre as impurezas substitucional e intersticial não é possível. Assim, as configurações obtidas a partir dos sítios AB- $O$ e $\mathrm{BC}$ poderiam existir unicamente se o dimero for incorporado à estrutura cristalina, entrando no lugar de uma vacância de arsênio.

Na Figura 3.5 mostramos os gráficos de contorno da densidade de carga total de valência para as configurações obtidas a partir dos sítios intersticiais AB-Ga e Hex. Nestas configurações as impurezas não interagem diretamente devido a seu afastamento relativo mas observamos uma interação com os átomos da rede mais próximos. Na Figura 3.5(a) mostramos a configuração final obtida a partir do sítio AB-Ga. Aqui vemos que o hidrogênio se liga ao gálio mais próximo, provocando o rompimento da ligação entre este e o oxigênio, sendo a distância de ligação $H-G a$ calculada em $1.62 \AA$. Entretanto, o oxigênio que agora tem coordenação três, desloca $0.4 \AA$ na direção $\langle 111\rangle$, ficando no plano formado pelos gálios com os quais continua ligado, e a distância de ligação $O-G a$ é de aproximadamente $2.0 \AA ̊$. Esta configuração apresenta uma energia total de $0.49 \mathrm{eV}$ maior que a configuração de mínima energia. Na Figura 3.5(b) mostramos a configuração de equilíbrio obtida a partir do sítio Hex. Nesta configuração os átomos do sistema não apresentam nenhum deslocamento importante, e o hidrogênio fica no seu sítio original fracamente ligado à rede. Este sistema apresenta a maior energia total entre as demais configurações chegando a $1.96 \mathrm{eV}$ acima da mais estável (C2).

Calculamos também a energia de ligação do átomo de hidrogênio para as diferentes configurações estudadas. Esta energia é definida como sendo a energia necessária para remover o átomo de hidrogênio do complexo $O_{A s}-H$, deixando-o afastado da impureza substitucional e ocupando um sítio intersticial na rede. Consideramos a formação deste complexo como originado na reação: $O_{A s}^{+}+H^{-} \rightarrow\left(O_{A s}-H\right)$, a qual seria esperada em um regime tipo- $p$ do material onde $O_{A s}^{+}$é a impureza mais estável (Figura 2.7). Desta forma a energia de ligação $E_{b}$ é dada por:

$$
-E_{b}=E_{t}\left(O_{A s}-H\right)-E_{t}\left(O_{A s}^{+}\right)+E_{t}\left(H_{T}^{-}\right)-E_{t}(G a A s),
$$

onde os dois primeiros termos da direita correspondem às energias totais do complexo neutro e da impureza substitucional de oxigênio positivamente carregada, respectivamente. $E_{t}\left(H^{-}\right)$é a energia total da impureza intersticial de hidrogênio no GaAs no estado de carga negativo, e sendo esta calculada no 

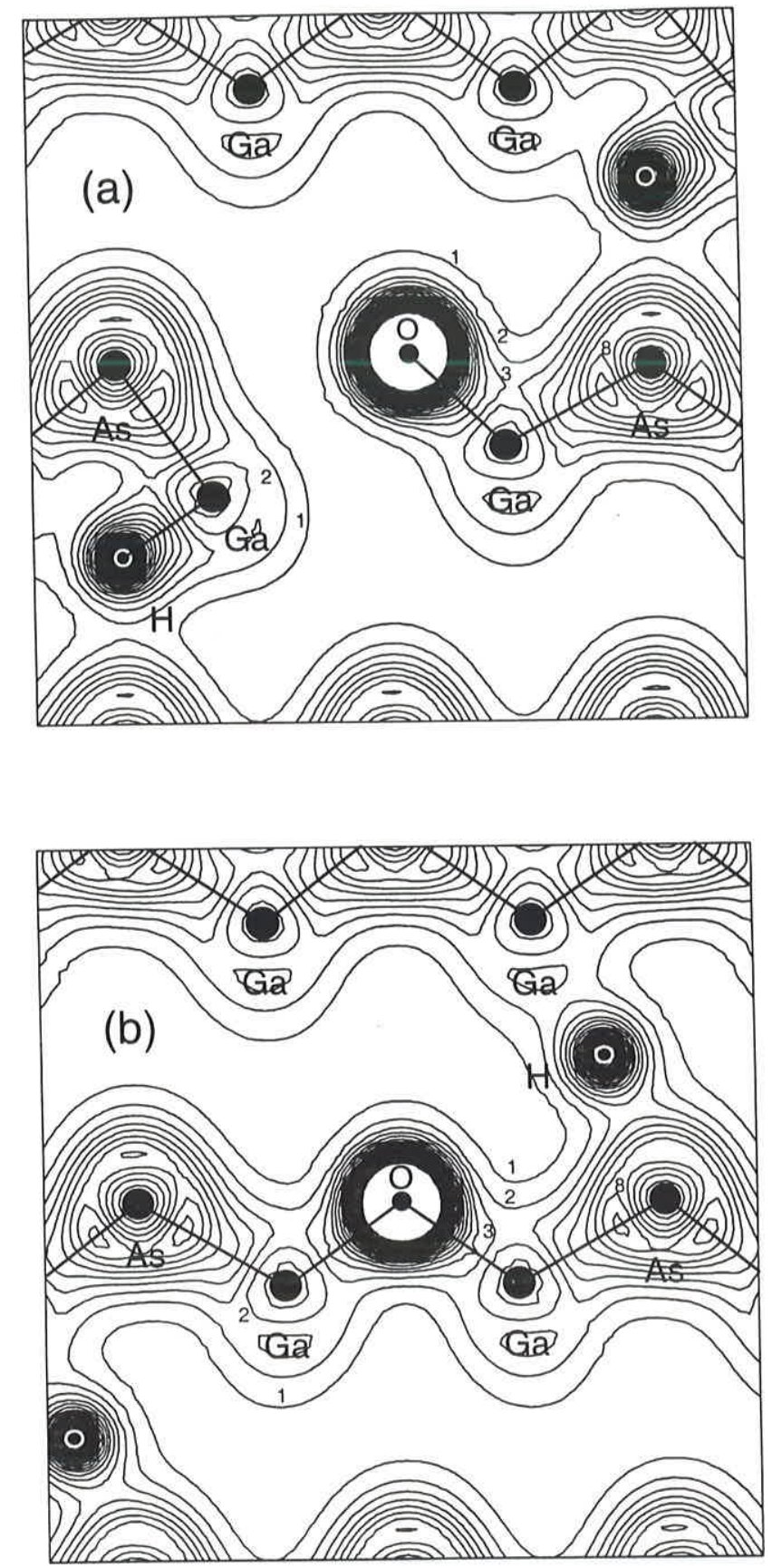

Figura 3.5: Gráficos de contorno da densidade de carga total de valência, através do plano [110], para duas configurações de equilíbrio do complexo $O_{A s}$ H em GaAs. (a) Configuração obtida a partir do sítio AB-Ga. (b) Configuração obtida a partir do sítio Hex. Unidades em $12.5 \mathrm{e} / \Omega$. 
Tabela 3.1: Geometria e energias do complexo $O_{A s}-H$ em GaAs para diferentes configurações de equilíbrio. $d_{O-H}$ representa a distância entre as impurezas de oxigênio e de hidrogênio e $d_{H-G a}$ a distância entre o hidrogênio $e$ o gálio primeiro vizinho. $\Delta E_{t}$ é a diferênça de energia total com respeito à configuração mais estável e $E_{b}$ a energia de ligação do hidrogênio.

\begin{tabular}{|c|c|c|c|c|}
\hline Configuração & $d_{O-H}[\AA]$ & $d_{H-G a}[\AA]$ & $\Delta E_{t}[\mathrm{eV}]$ & $E_{b}[\mathrm{eV}]$ \\
\hline \hline $\mathrm{AB}-G a$ & 3.00 & 1.62 & 0.49 & 1.91 \\
\hline $\mathrm{AB}-O$ & 1.04 & 2.24 & 1.37 & 1.03 \\
\hline $\mathrm{BC}$ & 1.05 & 1.94 & 0.36 & 2.03 \\
\hline $\mathrm{C} 1$ & 2.19 & 1.67 & 0.10 & 2.30 \\
\hline $\mathrm{C} 2$ & 1.88 & 1.54 & 0.00 & 2.40 \\
\hline $\mathrm{Hex}$ & 3.73 & 3.78 & 1.96 & 0.44 \\
\hline $\mathrm{T}$ & 2.21 & 1.62 & 0.17 & 2.23 \\
\hline
\end{tabular}

sítio T onde a interação com a rede é minimizada. $E_{t}(G a A s)$ é a energia total do cristal puro. Nossos resultados para a energia de ligação nas diferentes configurações estudadas, conjuntamente com as energias totais relativas à configuração mais estável ( $\mathrm{C} 2)$, e as distâncias entre os átomos mais próximos ao hidrogênio, são mostrados na Tabela 3.1.

Estimamos a contribuição da energia do ponto zero $\left(E_{P Z}\right)$ para as configurações onde o hidrogênio se liga ao oxigênio, a partir das freqüências vibracionais do radical $H O$ em álcool medidas experimentalmente, ${ }^{69}$ somando os termos $\frac{1}{2} \hbar \omega$ para os modos vibracionais mais relevantes. ${ }^{70}$ Esta estimativa é particularmente adequada para as configurações $\mathrm{BC}$ e $\mathrm{AB}-O$ onde são formadas ligações fortes $O-H$, assemelhando-se ao radical $O H$ e constitui um limite superior para as outras configurações onde a ligação $O-H$ é mais fraca. O valor calculado de $E_{P Z}$ é de $0.29 \mathrm{eV}$, e em rigor esta energia deveria ser subtraída da energia de ligação $E_{b}$ nas configurações AB- $O$ e BC. Para as configurações C1, C2 e T que apresentam similares características, suas energias $E_{P Z}$ devem ser muito próximas e certamente menores que $0.29 \mathrm{eV}$, devido a suas ligações $O-H$ serem mais fracas que nas configurações $\mathrm{BC}$ e AB-O. Desta forma, incluindo a energia do ponto zero nas configurações $\mathrm{BC}$ e $\mathrm{AB}-O$ vemos que $\mathrm{C} 2$ continua sendo a de menor energia e portanto a mais estável.

Calculamos ainda a estrutura eletrônica para três configurações finais do complexo $O_{A s}-H$ em $G a A s$, afim de verificar e caracterizar o processo de passivação do nível de defeito eletricamente ativo introduzido pela impureza 


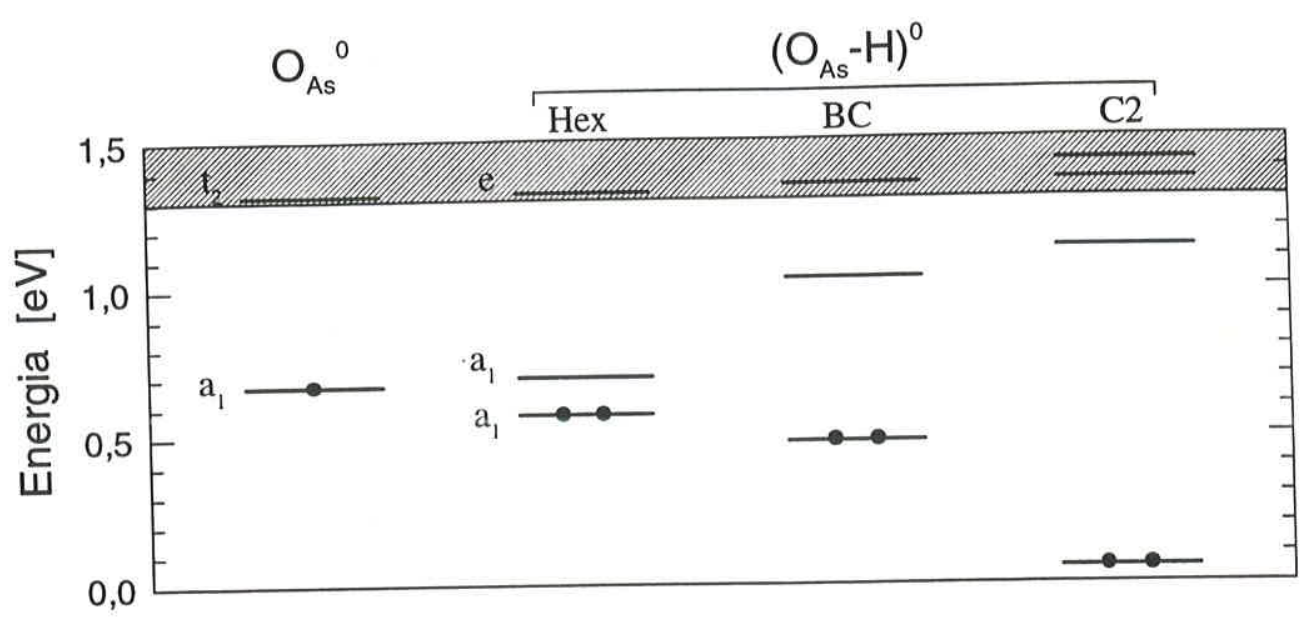

Figura 3.6: Representação esquemática dos autovalores de Kohn-Sham associados aos níveis de defeito no gap para o complexo $O_{A s}-H$ em GaAs, nas configurações de equilíbrio obtidas a partir dos sítios $\mathrm{Hex}, \mathrm{BC}$ e $\mathrm{C} 2$. Também é incluido o nível de defeito da impureza de oxigênio $O_{A s}$. Os círculos cheios indicam a ocupação nos níveis e a região hachurada representa o fundo da banda de condução.

de oxigênio. As configurações escolhidas foram as obtidas a partir dos sítios $\mathrm{Hex}, \mathrm{BC}$ e $\mathrm{C} 2$ que mostram interações diferentes entre as impurezas. No caso da configuração final Hex, esta representa uma situação onde não existe uma interação entre as impurezas, e o hidrogênio ocupa um posição intersticial interagindo fracamente com a rede. Na configuração final $\mathrm{BC}$, as impurezas interagem fortemente entre si e fracamente com a rede, mostrando uma característica molecular. Na configuração final $\mathrm{C} 2$, que é a de mínima energia, o hidrogênio interage medianamente tanto com a impureza de oxigênio como com um átomo da rede. As estruturas eletrônicas destas três configurações conjuntamente, com a da impureza de oxigênio, são mostradas esquematicamente na Figura 3.6. Aqui vemos que a passivação do nível de defeito do centro $O_{A s}$ em GaAs efetivamente ocorre, mas unicamente para a configuração mais estável (C2).

Nas configurações finais Hex e BC observamos dois níveis não degenerados na banda proibida e um nível bidegenerado no fundo da banda de condução. Devido ao átomo de hidrogênio nas configurações Hex e BC se localizar ao longo do eixo $\langle 111\rangle$, estes complexos possuem simetria pontual $C_{3 v}$, desta forma é possível atribuir os dois níveis observados a um desdobramento do nível $t_{2}$ do centro $O_{A s}$ de simetria $T_{d}$. $\mathrm{O}$ elétron adicionado pelo átomo de hidrogênio completa a ocupação do nível $a_{1}$ do defeito $O_{A s}$, deixando o 
complexo $O_{A s}-H$ eletricamente inativo, entretanto o nível $a_{1}$ vazio, também atribuido ao defeito $O_{A s}$, entra na banda proibida. Na configuração Hex, que representa o caso extremo onde hidrogênio não interage com oxigênio, os níveis $a_{1}$ se localizam aproximadamente no meio do gap separados em $0.15 \mathrm{eV}$. $\mathrm{Na}$ configuração $\mathrm{BC}$, que é o outro extremo onde as impurezas se ligam fortemente, os mesmos níveis tendem a se afastar chegando a uma separação de aproximadamente $0.5 \mathrm{eV}$. Finalmente na configuração mais estável $(\mathrm{C} 2)$, o nível $a_{1}$ ocupado desce até o topo da banda de valência e o nível $a_{1}$ vazio fica perto do fundo da banda de condução, aproximadamente $1.1 \mathrm{eV}$ acima do nível ocupado. Observamos que apesar das configurações $\mathrm{BC}$ e $\mathrm{C} 2$ possuirem energias totais muito próximas e serem suas geometrias similares, as estruturas eletrônicas mostram diferenças consideráveis, podendo serem explicadas de acordo com o tipo de hibridização entre os orbitais das impurezas e os átomos primeiros vizinhos. A formação da ligação forte entre o oxigênio e o hidrogênio observada na configuração $\mathrm{BC}$, pode ser interpretada como um dimero $\mathrm{OH}$ atuando como uma impureza substitucional de valência sete, a qual introduz dois orbitais pendentes quando susbtitui um arsênio, mantendo uma hibridização tipo $s p^{3}$. No caso da configuração C2, o oxigênio se liga mais fortemente a três gálios primeiros vizinhos, mostrando uma hibridização tipo $s p^{2}$, entretanto o orbital flutuante do quarto gálio é saturado pelo átomo de hidrogênio tendo uma participação pouco significativa na ligação com oxigênio. Estes sistemas são claramente diferentes o que explicaria as diferenças encontradas em suas respectivas estruturas eletrônicas. Isto também explicaria, no caso da configuração $\mathrm{C} 2$, a tendência dos níveis de impureza de sairem da banda proibida. Por último notamos que a introdução de hidrogênio no sistema da impureza substitucional $O_{A s}$ em $G a A s$ consegue neutralizar a atividade elétrica deste defeito mas não consegue remover totalmente os níveis da banda proibida. Como pode ser visto na Figura 3.5, a configuração mais estável para o complexo $O_{A s}-H$ é um duplo aceitador eletricamente inativo.

\subsection{Complexo $N_{A s}-H$ em $G a A s$}

Nesta seção apresentaremos um estudo da estrutura eletrônica e geometria atômica do complexo formado pela impureza isovalente de nitrogênio substituindo um arsênio e a impureza intersticial de hidrogênio em GaAs. Utilizamos os mesmos sítios intersticiais para o átomo de hidrogênio e o sítio substitucional de arsênio, definidos na seção anterior (Figura 3.1).

Nossos resultados mostram que a configuração de mínima energia, 


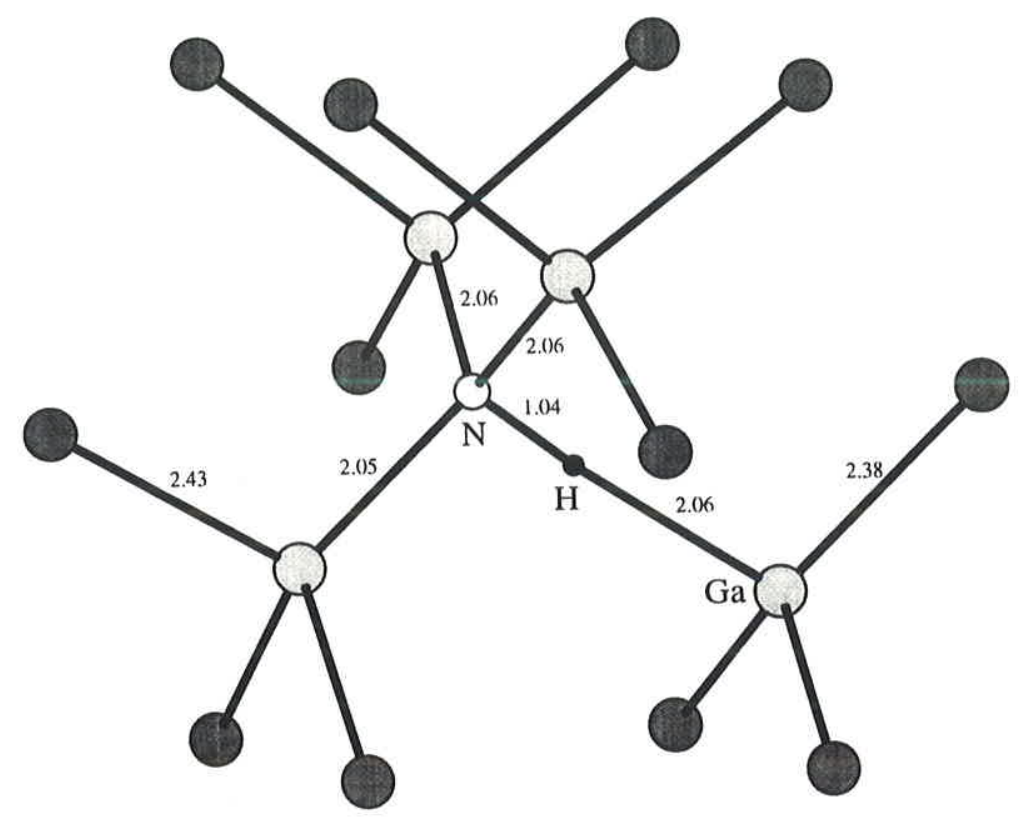

Figura 3.7: Configuração atômica de mínima energia obtida para o complexo $N_{A s}-H$ em GaAs. Os números representam as distâncias de ligação entre os átomos respectivos, em ångstroms.

tem o átomo de hidrogênio localizado em uma posição centrado na ligação $N$ $G a$ a qual conserva seu alinhamento. A distância de ligação $N-H$ é encontrada como sendo $1.04 \AA$, muito próxima do valor calculado na molécula de amônia $\left(\mathrm{NH}_{3}\right)$ que é de $1.03 \AA$. Esta configuração foi obtida a partir de dois sítios intersticiais onde foi colocado inicialmente o átomo de hidrogênio. Estes sítios são C1 e BC, conforme á Figura 3.1, no entanto a configuração obtida a partir do sítio $\mathrm{C} 1$ apresenta a menor energia para o complexo, e BC apresenta uma energia total de $0.23 \mathrm{eV}$ maior que $\mathrm{C} 1$. As posições dos átomos que são permitidos para relaxar na configuração mais estável são mostrados na Figura 3.7, onde são indicadas as distâncias de ligação atômicas mais relevantes. Aqui vemos que a inclusão do hidrogênio na ligação provoca um deslocamento do nitrogênio de $0.69 \AA ̊$ na direção de seus três gálios primeiros vizinhos, enquanto que o gálio ligado ao hidrogênio desloca se 0.31 Å na direção contrária. A configuração que apresenta a terceira menor energia total em relação ao sistema mais estável tem o hidrogênio ligado ao nitrogênio em uma posição antiligante, obtida a partir da posição inicial AB- $N$, e possui uma energia total $1.15 \mathrm{eV}$ maior que a configuração de mínimo. O átomo de nitrogênio e seus gálios primeiros vizinhos não apresentam nenhum deslocamento considerável com a 

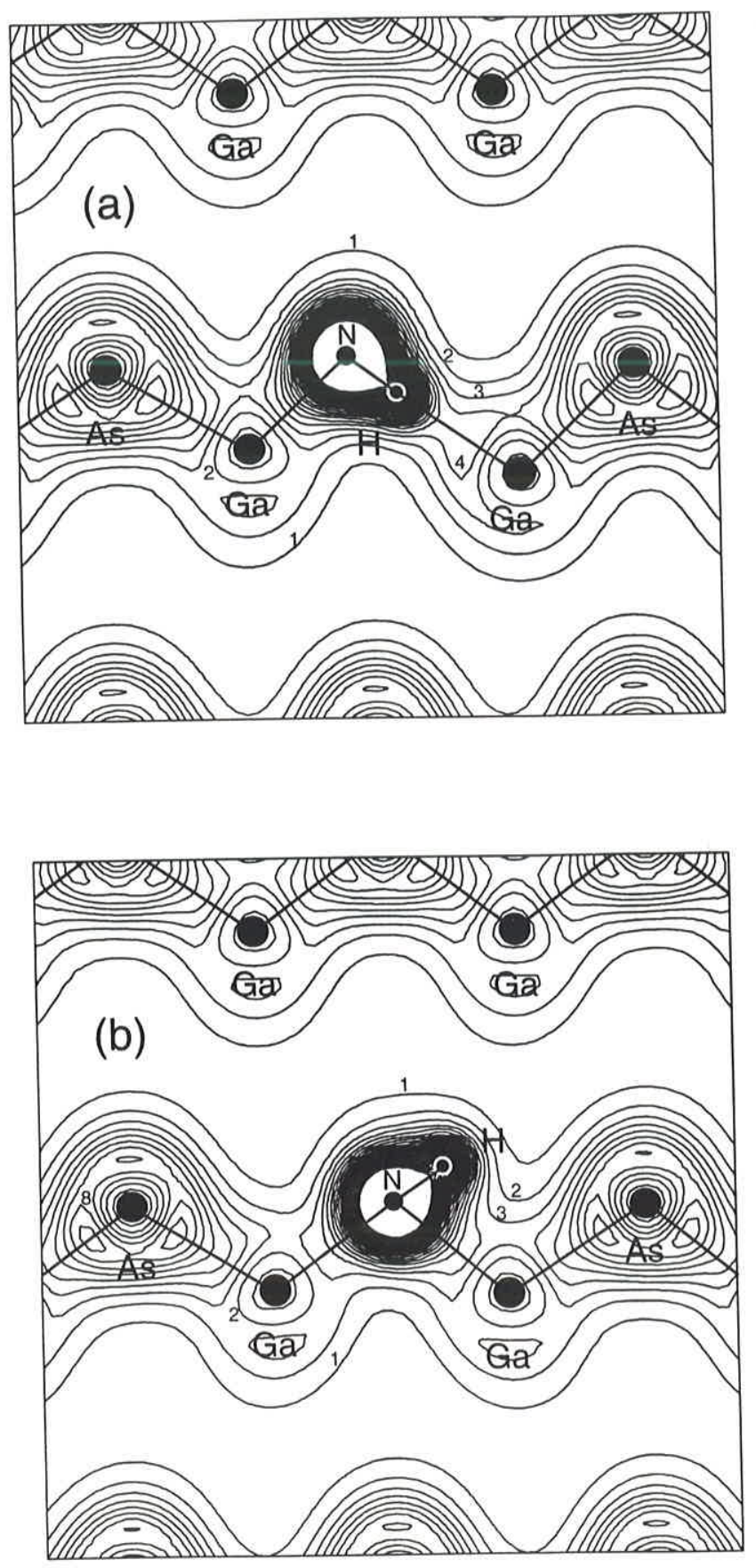

Figura 3.8: Gráficos de contorno da densidade de carga total de valência através do plano [110], para as configurações de menor energia do complexo $N_{A s}-H$ em GaAs. (a) Configuração obtida a partir do sítio C1. (b) Configuração obtida a partir do sitio AB- $N$. Unidades em $11.6 \mathrm{e} / \Omega$. 
incorporação do hidrogênio.

Na Figura 3.8 são mostrados os gáficos de contorno da densidade de carga total para as configurações obtidas a partir dos sítios $\mathrm{C} 1$ e AB- $N$. Aqui vemos que para estas duas configurações a situação mais favorável é a formação de uma ligação forte $N-H$, que interage mais fracamente com a rede. A configuração mais estável, Figura 3.8(a), mostra similares características com a configuração BC do complexo $O_{A s}-H$. O fato desta ser a configuração de mínima energia é devido a ausência de orbitais flutuantes, já que nitrogênio é uma impureza isovalente $\left(N_{A s}\right)$. Assim, o elétron do hidrogênio é atraido pela forte eletronegatividade de nitrogênio ligando-se a ele. É importante notar que a configuração de mínima energia foi obtida a partir de dois sítios diferentes, o proprio sítio centrado na ligação e um sítio puramente intersticial, i.e., longe de qualquer ligação. Em contraste, no caso do complexo $O_{A s}-H$, o hidrogênio prefere saturar o orbital flutuante introduzido pela impureza $O_{A s}$ e se ligar mais fracamente ao oxigênio, que é a configuração obtida a partir dos sítios puramente intersticiais. A formação da configuração centrada na ligação da Figura 3.4(a) é uma situação forçada e por isto que apresenta uma energia maior que a configuração mais estável. A distância de ligação $N-H$ encontrada é de $1.07 \AA$. A segunda configuração mais estável para o complexo $N_{A s}-H$, Figura 3.8(b), confirma a hipotése do hidrogênio de ligar fortemente ao nitrogênio pela ausência de orbitais flutuantes.

Na Figura 3.9 mostramos os gráficos de contorno da densidade de carga total para as configurações finais obtidas a partir dos sítios AB-Ga e T. Nestas vemos que o hidrogênio se liga fracamente à rede, interagindo com um arsênio segundo vizinho do nitrogênio. Na configuração AB-Ga, Figura 3.9(a), notamos uma clara diferença com respeito à mesma configuração do complexo $\mathrm{O}_{A s}-H$, Figura 3.5(a), onde existe uma quebra da ligação $G a-H$. Esta quebra é produzida pelo excesso de elétrons na impureza de oxigênio, que com a chegada do hidrogênio prefere perder um eletron de uma das ligações com gálio e se ligar aos outros três, mudando para uma hibridização $s p^{2}$, com o hidrogênio completando a ligação flutuante do gálio. No caso do complexo $N_{A s}-H$ nada disto ocorre devido ao fato de todas as ligações estarem completas. Na Figura 3.9(b) mostramos a configuração obtida a partir do sítio T. Nesta configuração observamos que hidrogênio não consegue chegar suficientemente perto do nitrogênio como para formar uma ligação, devido a interação com o arsênio mais próximo, ficando fracamente ligado à rede e conservando sua posição original. Os átomos mais próximos dele não sofrem deslocamentos consideráveis, e a energia total deste sistema é $1.63 \mathrm{eV}$ maior que a configuração de mínima energia. A configuração final obtida a partir do sítio C2 mostra o 



Figura 3.9: Gráficos de contorno da densidade de carga total de valência, através do plano [110], para duas configurações do complexo $N_{A s}-H$ em GaAs. (a) Configuração obtida a partir do sítio AB-Ga. (b) Configuração obtida a partir do sítio T. Unidades em $11.6 \mathrm{e} / \Omega$. 
Tabela 3.2: Geometria e energias do complexo $N_{A s}-H$ em GaAs para diferentes configurações de equilíbrio. $d_{N-H}$ representa a distância entre as impurezas de nitrogênio e hidrogênio e $d_{H-G a}$ a distância entre o hidrogênio e o gálio primeiro vizinho. $\Delta E_{t}$ é a diferênça de energia total com respeito à configuração mais estável e $E_{b}$ a energia de ligação do átomo de hidrogênio.

\begin{tabular}{|c|c|c|c|c|}
\hline Configuração & $d_{N-H}[\AA]$ & $d_{H-G a}[\AA]$ & $\Delta E_{t}[\mathrm{eV}]$ & $E_{b}[\mathrm{eV}]$ \\
\hline \hline $\mathrm{AB}-G a$ & 4.08 & 1.95 & 1.70 & -0.01 \\
\hline $\mathrm{AB}-N$ & 1.07 & 2.18 & 1.15 & 0.54 \\
\hline $\mathrm{BC}$ & 1.12 & 1.94 & 0.23 & 1.46 \\
\hline $\mathrm{C} 1$ & 1.04 & 2.06 & 0.00 & 1.69 \\
\hline $\mathrm{C} 2$ & 2.31 & 1.76 & 1.52 & 0.17 \\
\hline $\mathrm{Hex}$ & 3.65 & 3.55 & 1.57 & 0.12 \\
\hline $\mathrm{T}$ & 2.51 & 2.65 & 1.63 & 0.06 \\
\hline
\end{tabular}

hidrogênio ligado fracamente a um gálio e a um arsênio, primeiro e segundo vizinhos do nitrogênio respectivamente, verificando a tendência observada na configuração T. As distâncias de ligação $H$ - $A s$ e $G a-H$ são encontradas como sendo 1.81 e $1.76 \AA$ respectivamente. Esta configuração apresenta a maior energia total entre os diferentes sistemas estudados, $1.7 \mathrm{eV}$ maior que a configuração mais estável. Por último, na configuração final Hex o hidrogênio fica na mesma posição original, fracamente ligado à rede, apresentando as mesmas características que no complexo $O_{A s}-H$, mostrado na Figura 3.5(b).

Calculamos a energia de ligação para o átomo de hidrogênio considerando a formação do complexo como originado da reação das impurezas neutras: $N_{A s}^{0}+H^{0} \rightarrow\left(N_{A s}-H\right)$ onde $H^{0}$ é a impureza intersticial de hidrogênio localizada no sítio tetraédrico $(\mathrm{T})$. Esta reação seria a esperada considerando que a impureza substitucional $N_{A s}^{(0}$ em GaAs apresenta a menor energia de formação entre os diferentes estados de carga das impurezas $N_{A s}$ e $N_{G a}$ para um regime tipo- $p$ do material, tanto em um ambiente rico em átomos de gálio como de arsênio (Figura 2.19), sendo portanto a mais favorável de se formar. Escrevemos a energia de ligação do átomo de hidrogênio no complexo $N_{A s}-H$ como:

$$
-E_{b}=E_{t}\left(N_{A s}-H\right)-E_{t}\left(N_{A s}^{0}\right)+E_{t}\left(H_{T}^{0}\right)-E_{t}(G a A s),
$$




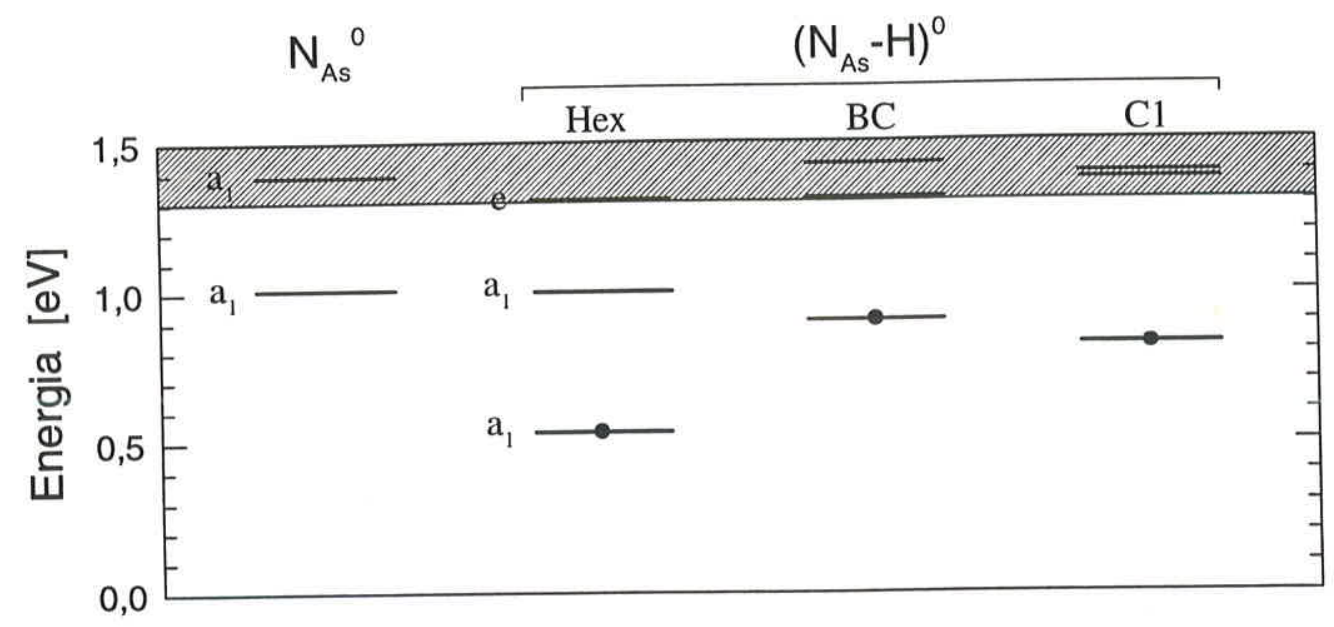

Figura 3.10: Representação esquemática dos autovalores de Kohn-Sham associados aos niveis de defeito no gap para o complexo $N_{A s}-H$ em GaAs, nas configurações de equilíbrio obtidas a partir dos sítios $\mathrm{Hex}, \mathrm{BC}$ e $\mathrm{C} 1$. Também são incluidos os níveis de defeito da impureza $N_{A s}^{0}$. Os círculos cheios indicam a ocupação nos níveis e a região hachurada representa o fundo da banda de condução.

onde $E_{t}$ representa a energia total dos respectivos sistemas definidos da mesma forma que na equação (3.1). Os resultados da energia de ligação do hidrogênio, energia total com respeito à configuração de mínima energia e as distâncias atômi-cas mais relevantes, para as diferentes configurações estudadas, são mostrada na Tabela 3.2. Da mesma forma que no complexo $O_{A s}-H$, estimamos a energia do ponto zero para as configurações onde o hidrogênio forma uma ligação com nitrogênio, a partir de medidas experimentais das freqüências de vibração do radical $\mathrm{NH}$ em amidos. ${ }^{69}$ A energia do ponto zero, neste caso, foi estimada em $0.24 \mathrm{eV}$ e deveria ser subtraída da energia de ligação dos complexos onde é formada uma ligação forte $N-H$, o que precisamente corresponde à configuração mais estável. As configurações onde o hidrogênio ocupa sítios puramente substitucionais ou fracamente ligado a algum átomo, tais como as configurações $\mathrm{AB}-G a, \mathrm{~T}$ e Hex, a energia do ponto zero é nula visto que não é formado um sistema vibracional envolvendo o hidrogênio.

Na Figura 3.10 mostramos uma representação esquemática dos níveis da banda proibida para três configurações de equilíbrio, incluindo a de mínima energia (C1), conjuntamente com a estrutura eletrônica da impureza de nitrogênio substitucional em $G a A s$ no estado de carga neutro $\left(N_{A s}^{(0)}\right)$. O complexo $N_{A s}-H$ para a configuração de mínima energia (C1), mostra um nível de sime- 
tria $a_{1}$ ocupado com um elétron, localizado a $0.82 \mathrm{eV}$ acima do topo da banda de valência, com os níveis superiores se localizando na banda de condução. Este nível eletricamente ativo é originado no nível vazio introduzido pela impureza $N_{A s}^{0}$ aproximadamente a $1.0 \mathrm{eV}$ acima do topo da banda de valência, o qual recebe o elétron adicionado pela impureza intersticial de hidrogênio quando é formado o complexo $N_{A s}-H$. A configuração Hex, que representa o caso onde o hidrogênio ocupa um sítio substitucional sem interagir com nitrogênio, mostra dois níveis não degenerados na banda proibida e um nível bidegenerado no fundo da banda de condução, localizados a 0.5, 1.0 e $1.3 \mathrm{eV}$ acima do topo da banda de valência respectivamente. O nível $a_{1}$ mais baixo é ocupado com o elétron do hidrogênio. Nas configurações BC e C1, que são as duas de menor energia e onde são formadas as ligações fortes $N$ - $H$, os níveis do gap são deslocados em aproximadamente $0.4 \mathrm{eV}$ na direção da banda de condução, deixando o nível $a_{1}$ semi-ocupado aproximadamente no meio do gap. Desta forma o complexo $N_{A s}-H$ em GaAs, no estado de carga neutro, é um centro aceitador profundo induzido pela incorporação do átomo de hidrogênio. É importante notar que a simples introdução do hidrogênio provoca a ativação elétrica do centro $N_{A s}$ sem necessáriamente interagir diretamente com nitrogênio.

\subsection{Complexo $N_{G a}-H$ em $G a A s$}

A formação do complexo $N_{G a}-H$ na sua configuração mais estável foi estudada seguindo a mesma metodologia das seções anteriores, i.e., incorporando o átomo de hidrogênio nos diferentes sítios intersticiais da Figura 3.1 e comparando suas energias totais, onde agora o sítio substitucional é um gálio.

Nossos resultados mostram que a configuração de mínima energia tem o átomo de hidrogênio localizado na posição centrada na ligação $N_{G a}$ - $A s$, fortemente ligado ao nitrogênio, de forma similar ao complexo $N_{A s}-H$. Esta configuração foi obtida a partir dos sítios $\mathrm{BC}, \mathrm{C} 1$ e $\mathrm{C} 2$, independentemente, onde as configurações finais $\mathrm{BC}$ e $\mathrm{C} 1$ apresentam praticamente a mesma energia total enquanto que a $\mathrm{C} 2$ é $0.11 \mathrm{eV}$ mais alta. As posições atômicas da configuração mais estável do complexo $N_{G a}-H$ são mostradas na Figura 3.11 onde também são indicadas as distâncias de ligação mais importantes.

A incorporação do hidrogênio na ligação $N$ - As provoca um deslocamento do nitrogênio do sítio substitucional de $0.56 \AA$ na direção de seus três arsênios primeiros vizinhos, ficando no mesmo plano, enquanto que o quarto arsênio ligado ao hidrogênio se desloca em aproximadamente $0.1 \AA$ na direção oposta. As distâncias de ligação de equilíbrio $N-H$ e $H$-As são encontradas 


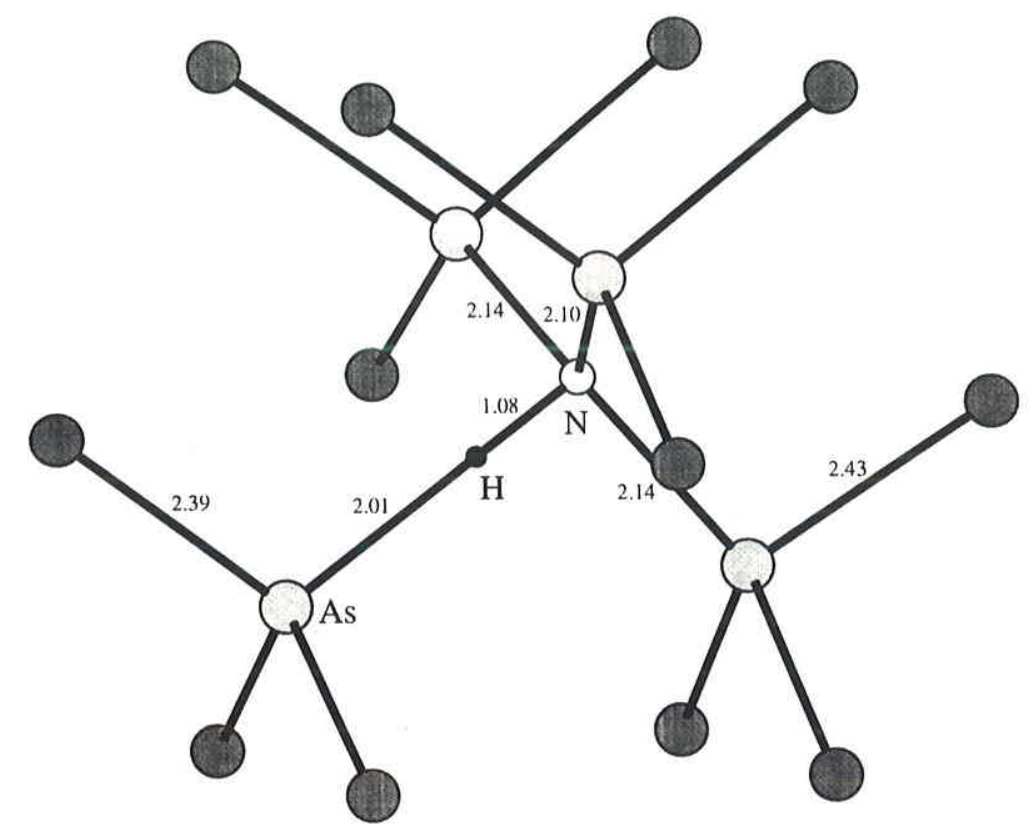

Figura 3.11: Configuração atômica de minima energia obtida para o complexo $N_{G a}-H$ em GaAs. Os números representam as distâncias de ligação entre os átomos respectivos, em ångstroms.

como sendo 1.08 e $2.01 \AA$, respectivamente, as quais são praticamente iguais as distâncias de ligação $N-H$ e $H-G a$ no complexo $N_{A s}-H$ (1.07 e $2.06 \AA$ respectivamente).

A Configuração que apresenta a segunda menor energia total tem o hidrogênio localizado na posição antiligante respeito do nitrogênio. Esta configuração foi obtida a partir do sítio AB- $N$ (AB-O na Figura 3.1) e possui uma energia $0.34 \mathrm{eV}$ maior que a configuração mais estável (BC). O Nitrogênio forma uma ligação com o hidrogênio ao longo da direção $\langle 111\rangle$, com uma distância de ligação $N$-H de $1.07 \AA$. Diferindo da configuração AB-O do complexo $O_{A s}-H$, o nitrogênio desloca do sítio substitucional na direção $\langle 111\rangle \mathrm{em}$ $0.79 \AA$. Este deslocamento pode ser explicado se considerarmos a metaestabilidade estrutural que apresenta a impureza $N_{G a}^{0}$ na direção $\langle 111\rangle$, e a presença do hidrogênio na posição antiligante $(\mathrm{AB}-N)$ favoreceria a ocupação do sítio metaestável por parte do nitrogênio no momento da formação do complexo.

Na Figura 3.12 são mostrados os gráficos de contorno da densidade de carga total de valência das configurações $\mathrm{BC}$ e $\mathrm{AB}-N$. Aqui vemos que ambas configurações apresentam o mesmo tipo de ligação forte para o par $N$ - $H$ observada no complexo $N_{A s}-H$. Na Figura 3.12(a) mostramos a densidade de carga 

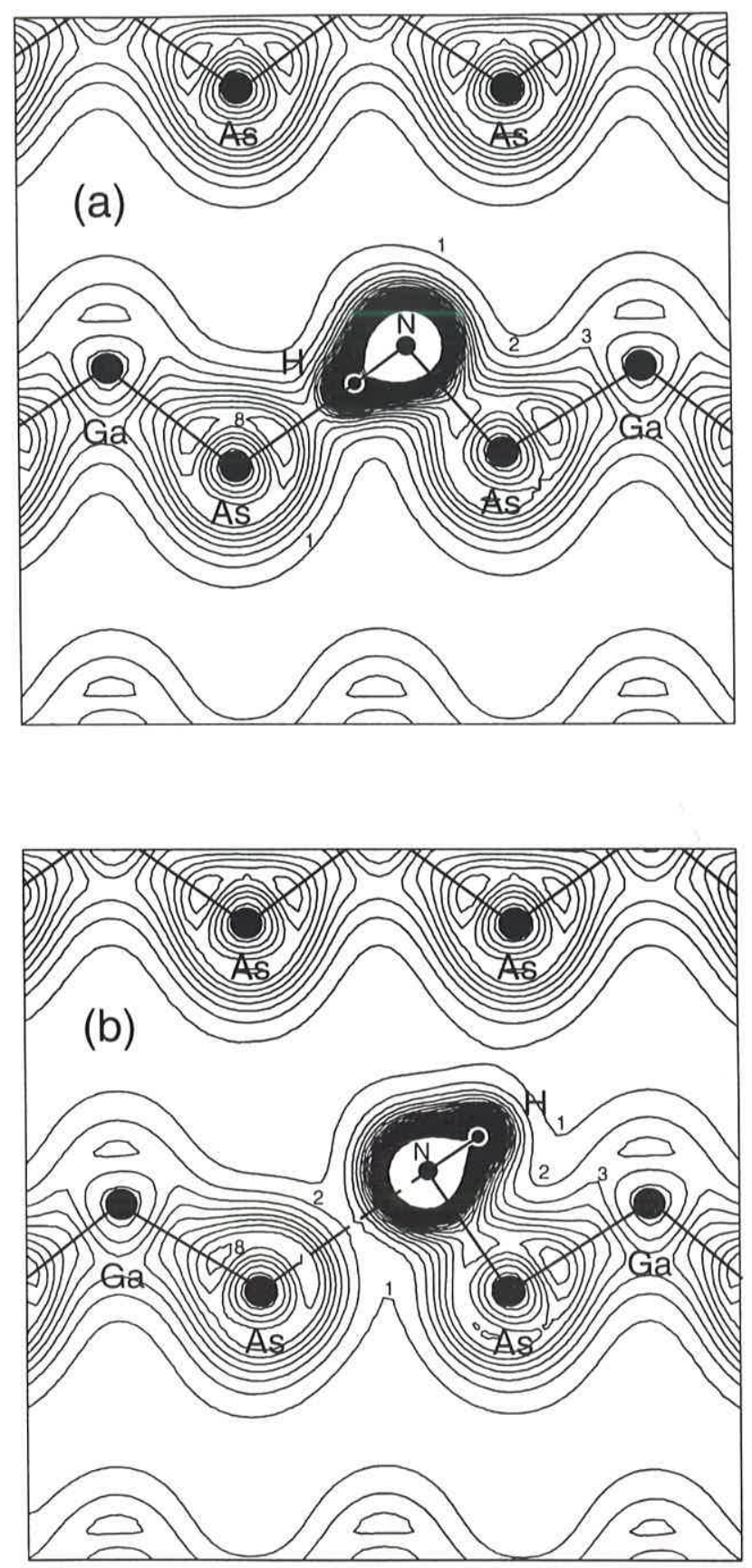

Figura 3.12: Gráficos de contorno da densidade de carga total de valência, através do plano [110], para as duas configurações de menor energia do complexo $N_{G a}-H$ em GaAs. (a) Configuração obtida a partir do sítio BC. (b) Configuração obtida a partir do sítio $\mathrm{AB}-N$. Unidades em $13.4 \mathrm{e} / \Omega$. 
da configuração de mínima energia. Aqui notamos a formação da molécula $N H$ que neste caso se liga mais fortemente à rede que na configuração $\mathrm{BC}$ do complexo $N_{A s}-H$. Na Figura 3.12(b) mostramos a densidade de carga para a configuração com a segunda menor energia total (AB- $N)$. Nesta configuração vemos que o hidrogênio se liga fortemente ao nitrogênio quebrando a ligação com um arsênio. Se comparamos com a Figura 2.14 que mostra a metaestabilidade da impureza de nitrogênio, vemos que suas distribuições de carga total são similares, confirmando que na formação do complexo com o hidrogênio, o nitrogênio ocupa o sítio metaestável. A distância entre o nitrogênio e o arsênio não ligado é de aproximadamente $3.0 \AA$.

$\mathrm{Na}$ Figura 3.13 mostramos as densidades de carga total de valência das configurações obtidas a partir dos sítios $\mathrm{AB}-A s$ e T. A configuração ABAs apresenta uma energia total $1.13 \mathrm{eV}$ maior com respeito à configuração de mínima energia (BC). Na Figura 3.13(a) observamos a formação da ligação $H$-As, com um comprimento de $1.56 \AA$, onde o hidrogênio atua como saturador. Entretanto, uma das ligações $N$-As é quebrada deixando o nitrogênio ligado a três arsênios, confirmando a tendência de mudar sua hibridização para $s p^{2}$, como também observada no estado metaestável da impureza $N_{G a}^{0}$. Na Figura 3.13(b) mostramos a densidade de carga obtida a partir da configuração T. Vemos que, da mesma forma que no complexo $N_{A s}-H$, o hidrogênio ocupa um sítio puramente intersticial, interagindo fracamente com a rede e não provocando deslocamentos importantes nos átomos mais próximos. A energia desta configuração é $2.53 \mathrm{eV}$ maior que a configuração mais estável. Finalmente a configuração obtida a partir do sítio Hex, cuja densidade de carga não mostramos, apresenta o hidrogênio na mesma posição na qual é deixado inicialmente, interagindo fracamente com a rede e de forma similar à configuração T.

A energia de ligação do átomo de hidrogênio no complexo $N_{G a}-H$, para as sete configurações de equilíbrio, foi cálculada a partir da reação entre impurezas neutras: $N_{G a}^{(0}+H^{0} \rightarrow\left(N_{G a}-H\right)$, devido ao centro $N_{G a}^{0}$ ser aquele que apresenta a menor energia de formação entre outros estados de carga da mesma impureza, para um regime tipo- $p$ do material, isto tanto para um ambiente rico em átomos de gálio como de arsênio (Figura 2.19). A energia de ligação foi obtida a partir da equação (3.2), considerando agora a impureza de nitrogênio substituindo um gálio. Nossos resultados são mostrados na Tabela 3.3 onde também é mostrado a energia total de cada configuração relativa à configuração mais estável e as distâncias de ligação mais importantes. A contribuição da energia do ponto zero para as configurações que formam ligação forte $N-H$, que foi estimada a partir das energias vibracionais 

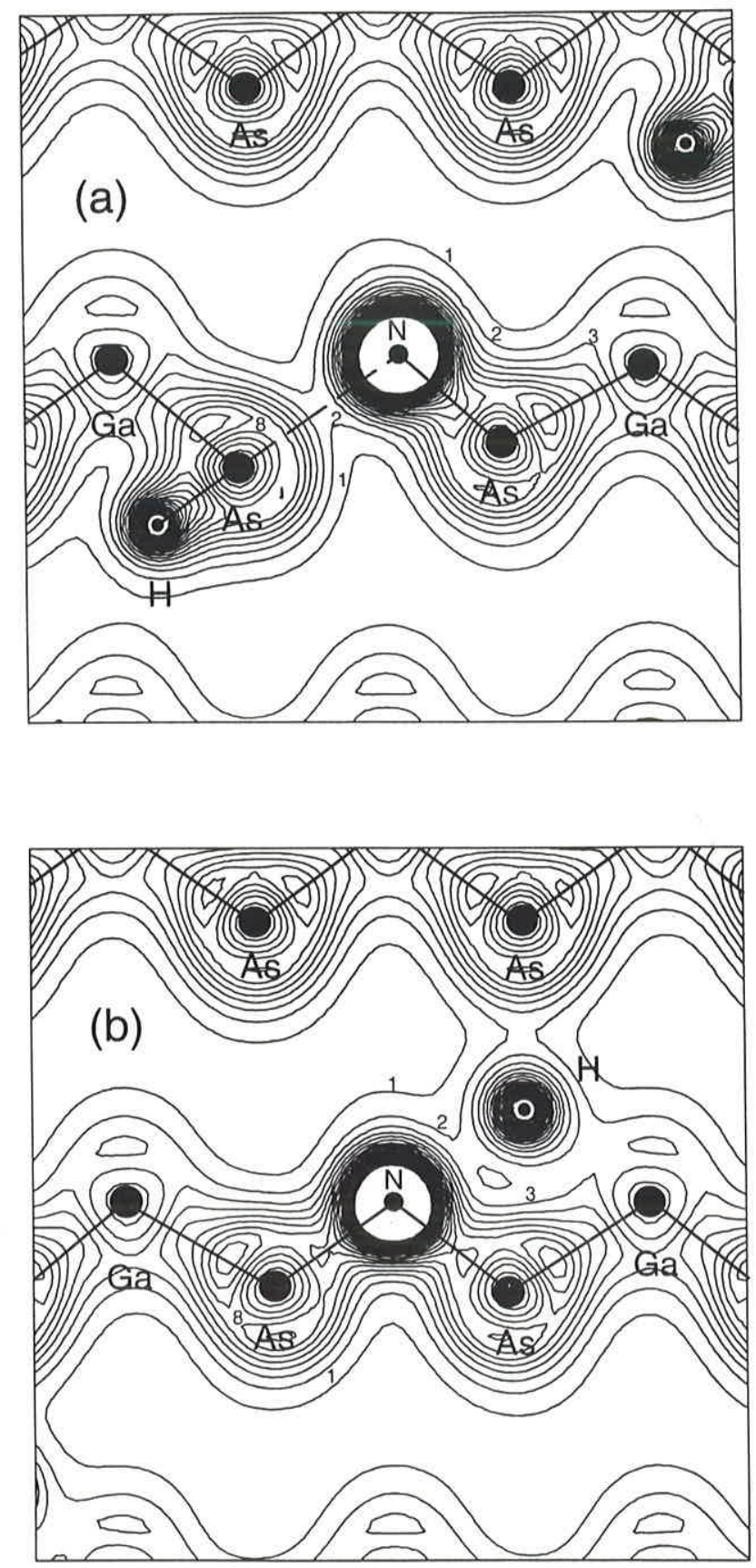

Figura 3.13: Gráficos de contorno da densidade de carga total de valência, através do plano [110], para duas configurações de equilíbrio do complexo $\mathrm{N}_{G a^{-}}$ $H$ em GaAs. (a) Configuração obtida a partir do sítio AB-As. (b) Configuração obtida a partir do sítio T. Unidades em $13.4 \mathrm{e} / \Omega$. 
Tabela 3.3: Geometria e energias do complexo $N_{G a}-H$ em GaAs para diferentes configurações de equilibrio. $d_{N-H}$ representa a distância entre as impurezas de nitrogênio e de hidrogênio e $d_{H-A s}$ a distância entre o hidrogênio $e$ $o$ arsênio primeiro vizinho. $\Delta E_{t}$ é a diferênça de energia total com respeito à configuração mais estável e $E_{b}$ a energia de ligação do átomo de hidrogênio.

\begin{tabular}{|c|c|c|c|c|}
\hline Configuração & $d_{N-H}[\AA]$ & $d_{H-A s}[\AA]$ & $\Delta E_{t}[\mathrm{eV}]$ & $E_{b}[\mathrm{eV}]$ \\
\hline \hline $\mathrm{AB}-A s$ & 4.46 & 1.56 & 1.13 & 1.65 \\
\hline $\mathrm{AB}-N$ & 1.06 & 2.41 & 0.34 & 2.45 \\
\hline $\mathrm{BC}$ & 1.08 & 2.01 & 0.00 & 2.79 \\
\hline $\mathrm{C} 1$ & 1.07 & 2.02 & 0.01 & 2.78 \\
\hline $\mathrm{C} 2$ & 1.08 & 1.96 & 0.11 & 2.67 \\
\hline $\mathrm{Hex}$ & 3.59 & 3.58 & 2.66 & 0.13 \\
\hline $\mathrm{T}$ & 2.39 & 2.69 & 2.53 & 0.26 \\
\hline
\end{tabular}

da molécula $N H$ na seção anterior, e que também deve ser incluida na energia de ligação do átomo de hidrogênio no complexo $N_{G a}-H$, foi calculada como sendo $0.24 \mathrm{eV}$. Na Tabela 3.3 notamos que as energias de ligação do hidrogênio nas configurações mais estáveis do complexo $N_{G a}-H$, são da ordem de $1.0 \mathrm{eV}$ maiores que no complexo $N_{A s}-H$ (Tabela 3.2). Isto é devido aos dois orbitais flutuantes introduzidos pela impureza $N_{G a}$ os quais se ligam mais fortemente ao hidrogênio no complexo, e no caso da impureza isovalentes estes orbitais flutuantes não existem, logo o hidrogênio fica mais fracamente ligado. Para a configuração Hex nos complexos $N_{A s}-H$ e $N_{G a}-H$, a energia de ligação do hidrogênio é praticamente a mesma, e isto é esperado considerando que nesta configuração o hidrogênio não interage com a impureza susbtitucional.

A estrutura eletrônica do complexo $N_{G a}-H$ em $G a A s$ para as duas configurações mais estáveis (AB- $N$ e BC), mostra três níveis na banda proibida, dois níveis não degenerados ocupados com dois e um elétrons na parte inferior do gap e um nível bidegenarado, vazio, na parte superior. Como o hidrogênio nestas duas configurações ocupa sítios ao longo do eixo $\langle 111\rangle$, a simetria pontual do complexo é $C_{3 v}$, e desta forma os níveis observados podem ser atribuidos ao desdobramento do nível $t_{2}$ introduzido pela impureza substitucional $N_{G a}^{0}$ na parte superior do gap. Na Figura 3.14 mostramos uma representação esquemática dos níveis da banda proibida para três configurações de equílibrio do complexo $N_{G a}-H$, os quais são comparados com a estrutura eletrônica obtida para o centro $N_{G a}^{0}$ em $G a A s$. Aqui vemos que as três con- 


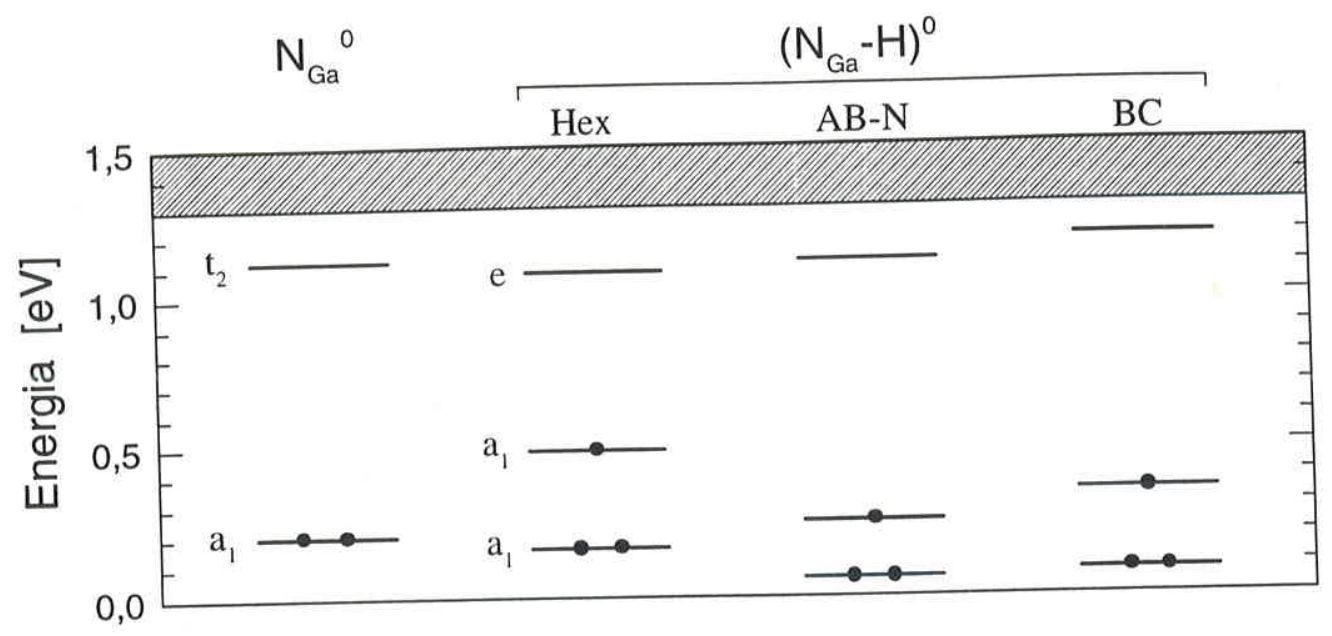

Figura 3.14: Representação esquemática dos autovalores de Kohn-Sham associados aos niveis de defeito no gap para o complexo $\mathrm{N}_{G a}-\mathrm{H}$ em GaAs, nas configurações de equilíbrio obtidas a partir dos sítios $\mathrm{Hex}, \mathrm{AB}-N$ e $\mathrm{BC}$. Também são incluidos os níveis de defeito da impureza $N_{G a}^{0}$. Os círculos cheios indicam a ocupação nos níveis e a região hachurada representa o fundo da banda de condução.

figurações apresentam o mesmo número de níveis no gap variando levemente suas posições, e é importante lembrar que na configuração Hex não existe uma interação entre as impurezas. O nível $a_{1}$ ocupado com dois elétrons, o qual é originado na impureza substitucional, conserva aproximadamente a mesma característica no complexo, entretanto os dois níveis superiores $\left(a_{1}\right.$ e $e$ ) aumentam seu espaçamento em aproximadamente $0.3 \mathrm{eV}$ quando o hidrogênio passa de uma posição intersticial a uma onde forma uma ligação forte com nitrogênio. Notamos que a estrutura eletrônica da configuração Hex é semelhante à obtida para a impureza $N_{G a}^{-}$(Figura 2.17), o que mostra que a introdução de elétrons ao sistema é o fato que provoca variações significativas na estrutura eletrônica do semicondutor. Finalmente verificamos que a formação do complexo $N_{G a}-H$ em GaAs é um centro aceitador profundo induzido pela incorporação do átomo de hidrogênio no sistema de impureza $N_{G a}^{0}$ em GaAs.

Neste capítulo estudamos a formação dos complexos envolvendo a impureza intersticial de hidrogênio e as impurezas substitucionais de oxigênio e nitrôgenio em GaAs. Os resultados obtidos para o complexo $O_{A s}-H$ na configuração mais estável mostram o hidrogênio em uma posição centrada na ligação, sendo esta não linear, formando um ângulo $O-H-G a$ de $160^{\circ}$. Os gráficos da densidade de carga total mostram que oxigênio se liga mais forte- 
mente a três gálios primeiros vizinhos ficando no mesmo plano destes, entretanto o hidrogênio se liga mais fortemente com o quarto gálio atuando como saturador, e em conseqüência a ligação $H-O$ é fraca. A energia de ligação do hidrogênio foi calculada a partir de uma reação entre as impurezas carregadas $O_{A s}^{+}$e $H^{-}$, favorável em um regime tipo- $p$ do material, e o valor obtido foi de $2.4 \mathrm{eV}$. Considerando que o hidrogênio se liga fracamente com oxigênio e gálio, acreditamos que a contribuição da energia do ponto zero na energia de ligação para esta configuração seja mínima. A estrutura eletrônica deste complexo mostra que hidrogênio passiva o nível eletricamente ativo introduzido pela impureza substitucional $O_{A s}$ no meio do gap, deslocando-o para o topo da banda de valência e deixando a banda proibida parcialmente limpa. Notamos também que a remoção dos níveis do gap somente ocorre para as configurações que apresentam menor energia. Atribuimos este fato à hibridização tipo $s p^{2}$ formada entre os orbitais do oxigênio e os três gálios primeiros vizinhos nestas configurações, onde o hidrogênio não teria uma participação direta.

Nossos resultados para complexo $N_{A s}-H$ em GaAs mostram que a configuração mais estável tem o hidrogênio localizado na posição centrada na ligação $N$-Ga, que neste caso é alinhada. Os gráficos de contorno da densidade de carga total para esta configuração mostram uma ligação forte $N$-H de $1.04 \AA$ de comprimento, que atribuimos à formação do dimero $N H$ o qual ocupa o lugar da vacância de arsênio ligando-se a rede e formando hibridização tipo $s p^{3}$. A energia de ligação do hidrogênio, que é calculada considerando uma reação entre as impurezas neutras $N_{A s}^{()}$e $H^{(0}$ favorável em um regime tipo-p, é de $1.69 \mathrm{eV}$. Nesta configuração a contribuição da energia do ponto zero é importante devido à formação da forte ligação $N-H$, tendo seu valor estimado a partir das freqüências de vibração do radical $N H$ em amidos. O valor encontrado é de $0.24 \mathrm{eV}$. Claramente este valor é superestimado, visto que $N H$ se liga mais fortemente nos amidos que no cristal GaAs onde são esperadas freqüências de vibração maiores. A estrutura eletrônica do complexo $N_{A s}-H$ mostra que este centro é eletricamente ativo, apresentando um nível semiocupado aproximadamente no meio da banda proibida. Comparando com a estrutura eletrônica da impureza substitucional isovalente $N_{A s}^{0}$, concluímos que a ativação ocorre pela ocupação de um nível vazio introduzido por este centro, onde o elétron é doado pelo hidrogênio quando é formado o complexo.

Finalmente para o complexo $N_{G a}-H$ a configuração de equilíbrio que apresenta a menor energia total tem o hidrogênio localizado no sítio centrado na ligação $N-A s$, em forma similar ao complexo $N_{A s}-H$. Os gráficos da densidade de carga total também mostram a formação da ligação forte $N-H$, que neste caso tem um comprimento de $1.08 \AA$. Uma diferença que notamos 
com respeito ao complexo $N_{A s}-H$ é que a metaestabilidade estrutural observada na impureza substitucional $N_{G a}^{0}$, a qual mostra ligações trigonalmente simétricas indicando uma hibridização tipo $s p^{2}$, influencia a formação de certas configurações nas quais são observadas quebras de ligações. A energia de ligação do átomo de hidrogênio foi calculada considerando uma reação entre as impurezas neutras $N_{G a}^{0}$ e $H^{0}$ favorável em um regime tipo- $p$ do material, sendo o valor obtido de $2.79 \mathrm{eV}$, aproximadamente $1.0 \mathrm{eV}$ maior que do complexo $N_{A s}-H$. O fato do hidrogênio estar mais fortemente ligado no complexo $N_{G a}-H$ tem a ver com os orbitais flutuantes que apresenta a impureza $N_{G a}^{0}$, os quais fortalecem a ligação do hidrogênio. Com respeito a estrutura eletrônica, nossos resultados mostram que o centro $N_{G a}^{0}$ também é ativado eletricamente com a presença do hidrogênio, o qual ocorre da mesma forma que no complexo $N_{A s}$-H, i.e., pela ocupação de um nível vazio introduzido pela impureza substitucional. O complexo $N_{G a}-H$ na sua configuração mais estável apresenta um nível $a_{1}$ ocupado com um elétron localizado aproximadamente a $0.4 \mathrm{eV}$ acima do topo da banda de valência. Desta forma, verificamos que centros inativos que apresentam níveis vazios ou totalmente ocupados na banda proibida, podem ser ativados eletricamente com a incorporação de hidrogênio quando são formados complexos. O mecanismo consiste na ocupação de níveis vazios não degenerados presentes na banda proibida, os quais são introduzidos pelas impurezas substitucionais. 


\section{Capítulo 4}

\section{Complexos de Nitrogênio e Pares de Hidrogênios em $G a A s$}

As propriedades estruturais e eletrônicas de múltiplas impurezas intersticiais de hidrogênio e sua interação com defeitos e dopantes em semicondutores, têm sido objeto de recentes pesquisas tanto do ponto de vista teórico como experimental. Os primeiros estudos teóricos envolvendo pares de átomos de hidrogênio em silício cristalino foram motivados pela controvérsia do estado de carga e da identidade do tipo de espécie que se difunde, i.e., se esta envolve hidrogênio atômico ou molecular. ${ }^{71}$ Estes trabalhos mostraram que o hidrogênio se difunde em forma atômica para posteriormente formar a molécula que é encontrada no sítio tetraédrico $(\mathrm{T})$ do cristal, orientada na direção $\langle 100\rangle{ }^{72.73}$ Não existe uma medição experimental direta da presença da molécula $\mathrm{H}_{2}$ em silício devido a esta ser inativa no infravermelho e possuir espectros Ramman de pouca resolução. Outros estudos teóricos têm proposto a formação de uma estrutura metaestável alternativa à molecular envolvendo dois hidrogênios, denotada por $H_{2}^{*}$. Nesta estrutura um dos hidrogênios ocupa um sítio centrado na ligação e o outro em um sítio antiligante em simetria trigonal $C_{3 v}$. Este complexo foi primeiramente proposto em diamante por Briddon et $a .^{74}$ e posteriormente em silício por Chang e Chadi. ${ }^{73}$ Medidas de modos locais de vibração no infravermelho, conjuntamente com cálculos teóricos das freqüências de vibração, têm confirmado a estrutura proposta para $H_{2}^{*}$ em silício ${ }^{75}$ e recentemente em germânio. ${ }^{76}$ Por outro lado, Pavesi e Giannozzi ${ }^{81}$ estudaram o hidrogênio molecular e o complexo $H_{2}^{*}$ em GaAs. Neste cálculo foi mostrado a formação da molécula $H_{2}$ cuja posição mais estável é no sítio T, mais próximo de um gálio e fracamente perturbada pela rede onde duas possíveis orientações foram encontrada com a mesma energia, $\langle 111\rangle$ e 
$\langle 100\rangle$. Também foi proposta a formação do defeito tipo $H_{2}^{*}$ o qual tem um dos hidrogênios centrado na ligação $A s-G a$ e o outro no sítio antiligante com respeito ao mesmo gálio, em simetria trigonal. Este defeito possui uma energia de $1.0 \mathrm{eV}$ a mais que a molécula. Recentemente foi demostrada a existência de hidrogênio molecular em GaAs o qual foi observado por meio de espectroscopia Ramman. $^{82}$ Nesta experiência $H_{2}$ mostra uma fraca interação com o cristal rodando em torno do sítio $\mathrm{T}$ do cristal, concordando com os cálculos teóricos acima mencionados.

Cálculos de primeiros princípios envolvendo impurezas rasas e pares de átomos de hidrogênio em silício têm mostrado que os dopantes conseguem capturar um dos hidrogênios, enquanto que o segundo hidrogênio fica ligado a átomos hospedeiros primeiros vizinhos. ${ }^{79.80}$ Em alguns casos foi observado a formação de estruturas tipo $H_{2}^{*}$, i.e., um dos hidrogênios ocupa uma posição centrada na ligação e o outro uma posição antiligante em simetria trigonal, onde nenhum deles se liga à impureza. ${ }^{78}$ Recentemente Amore Bonapasta ${ }^{65}$ tem efetuado cálculos de primeiros princípios de complexos envolvendo defeitos e pares de hidrogênios em GaAs. Nestes cálculos foi estudado a passivação do defeito EL2, que é um doador profundo, com a incorporação de dois hidrogênios formando um complexo do tipo $A s_{G a}-H_{2}^{*}$. Estudou aida as propriedades vibracionais e estrutrais deste mesmo complexo para dua configurações, a mais estável e uma metaestável. ${ }^{83}$

Estudos teórico de complexos envolvendo impurezas e pares de hidrogênios em $G a A s$ não tem sido até agora verificados na literatura. Recentemente foi publicado um trabalho experimental que estuda o espectro infravermelho de GaP dopado com nitrogênio e hidrogênio, ${ }^{15}$ onde um complexo trigonal envolvendo nitrogênio e um par de hidrogênios tem sido identificado. Os modos locais de vibração foram atribuidos a um defeito formado por um nitrogênio substituindo um arsênio e dois hidrogênios, localizados em sítios ligante e antiligante com respeito à impureza. A formação do mesmo complexo também foi identificada em $\mathrm{GaAs},{ }^{16}$ apresentando similares características.

Neste capítulo apresentaremos cálculos de energia total, estrutura eletrônica e configuração atômica para os complexos envolvendo a impureza substitucional de nitrogênio e pares de hidrogênios intersticiais em $\mathrm{GaAs}, \mathrm{N}_{\mathrm{As}}{ }^{-}$ $\mathrm{H}_{2}$ e $\mathrm{N}_{\mathrm{Ga}}-\mathrm{H}_{2}$. Estes cálculos foram realizados em uma célula de 32 átomos onde duas camadas atômicas em volta da impureza substitucional e as própias impurezas foram permitidas relaxar.

Para determinar a configuração mais estável, foram analisados seis diferentes sítios intersticiais para os hidrogênios conforme são ilustrados esquematicamente na Figura 4.1. Consideramos unicamente posições próximas entre

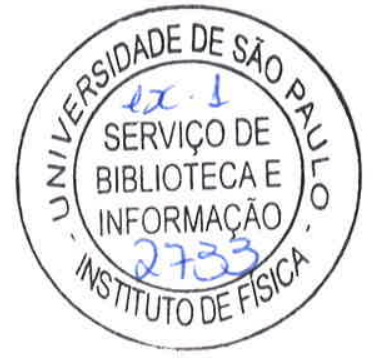



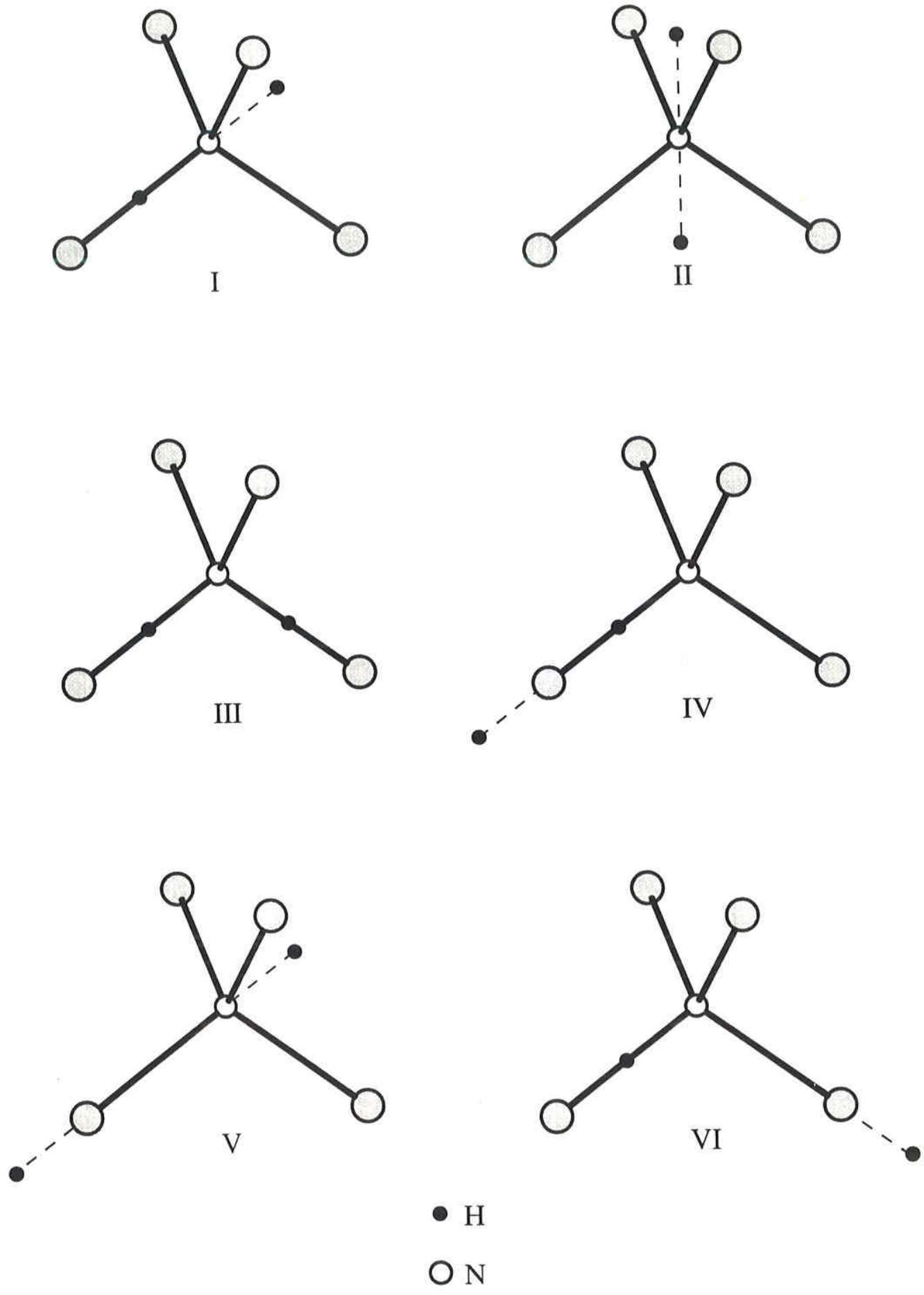

Figura 4.1: Representação esquemática dos sítios intersticiais dos átomos de hidrogênio utilizados como configurações iniciais no estudo da formação do complexo $\mathrm{N}-\mathrm{H}_{2}$ em $\mathrm{GaAs}$. 
as impurezas, deixando a posibilidade de um dos hidrogênios formar ligação com o nitrogênio, que é a configuração mais estável observada nos complexos envolvendo nitrogênio e um único hidrogênio, como vimos no capítulo anterior. Na Figura 4.1 as configurações I, IV e VI são definidas como do tipo $H_{2}^{*}$, com um hidrogênio centrado na ligação $N-G a$ e o outro em uma posição antiligante com respeito a um gálio ou ao nitrogênio, em simertria trigonal. As configurações III e V representam posições equivalentes, centrada na ligação e antiligante respectivamente. A configuração II representa uma situação especial onde não é forçada uma determinada ligação para os hidrogênios. Nosso objetivo é determinar a configuração de equilíbrio que apresenta a menor energia total entre as diferentes configurações aqui estudadas e a partir desta verificar a possível "repassivação" dos complexos que foram previamente ativados com a incorporação de um hidrogênio. Neste cálculo foi considerado uma energia de corte de 42 Ry na espansão da ondas planas, da mesma forma que os cálculos anteriores envolvendo o nitrogênio.

\subsection{Complexo $\mathrm{N}_{A s}-H_{2}$ em $\mathrm{GaAs}$}

A configuração mais estável do complexo $N_{A s}-H$ em $G a A s$, estudado no capítulo anterior, mostra o hidrogênio ocupando uma posição centrado na ligação, fortemente ligado ao nitrogênio, onde a ligação $N-H$ apresenta características de molécula. Nossos resultados para o complexo envolvendo agora dois hidrogênios $N_{A s}-H_{2}$, mostram que a configuração de menor energia tem um hidrogênio localizado na posição centrado na ligação enquanto que o segundo hidrogênio se localiza na posição antiligante com respeito ao nitrogênio, em simetria trigonal. Este sistema foi obtido a partir da configuração inicial I da Figura 4.1. Uma segunda configuração com praticamente a mesma energia total (0.04 eV maior que a configuração anterior), foi obtida a partir da configuração inicial VI da Figura 4.1. Nesta um dos hidrogênios se localiza na posição centrado na ligação e o outro antiligante com respeito a um dos gálio primeiros vizinhos, em simetria trigonal. Notamos que os dois complexos de menor energia formam estrutura tipo $H_{2}^{*}$.

Na Figura 4.2 mostramos as posições atômicas de equilíbrio destas duas configurações onde são indicadas as distâncias de ligação mais importantes. Na Figura 4.2(a) mostramos a configuração de menor energia, e vemos que é formada a ligação $N-H$ de $1.05 \AA$ de comprimento, muito próximo de valor teórico obtido na molécula de amônia $(1.03 \AA$ ). A incorporação do hidrogênio centrado na ligação provoca um grande deslocamento do nitrogênio 
(a)

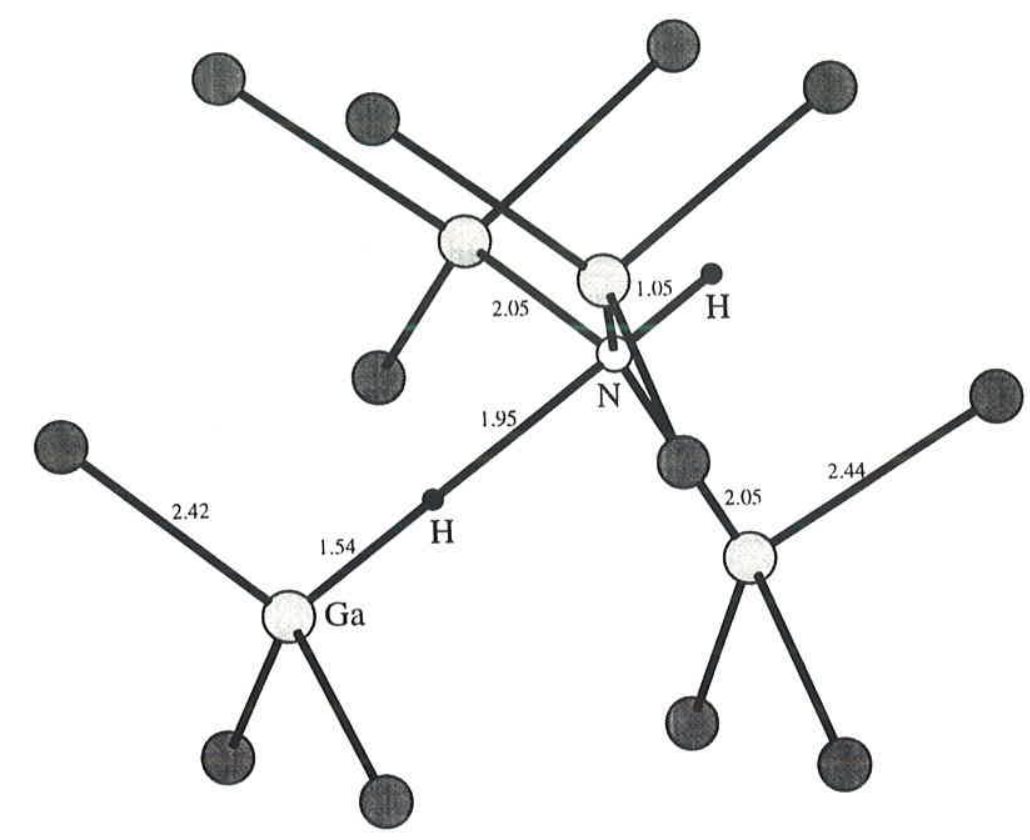

(b)

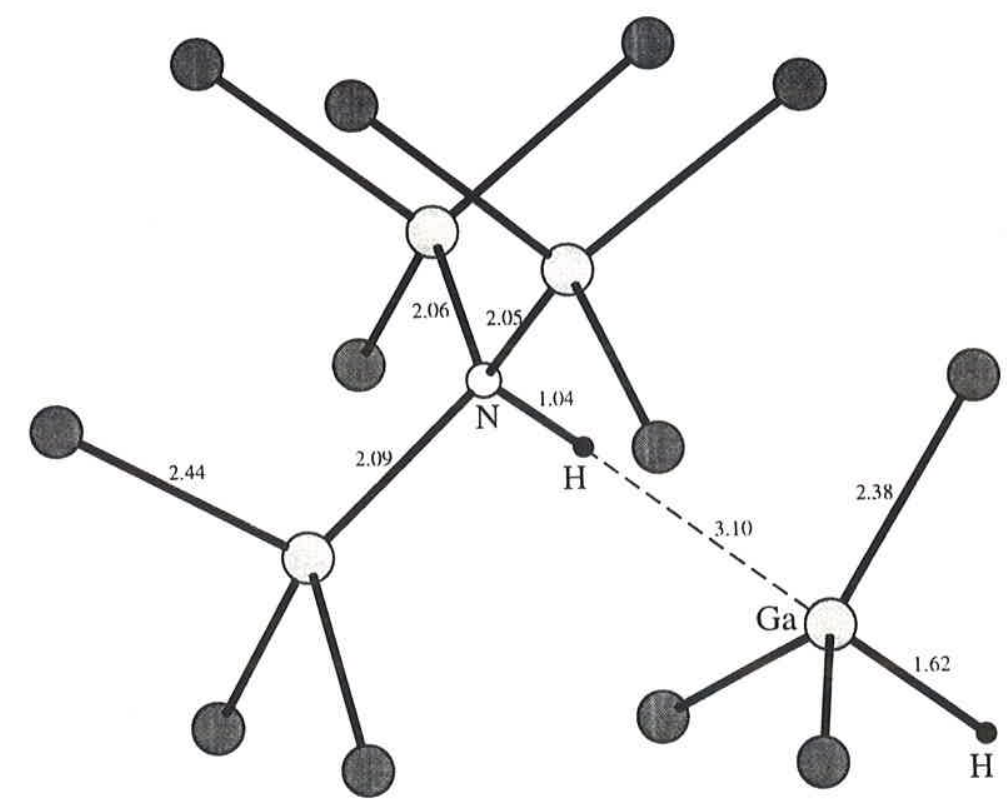

Figura 4.2: Configurações atômicas de menor energia obtidas para o complexo $\mathrm{N}_{\mathrm{As}}-\mathrm{H}_{2}$ em GaAs. (a) Configuração mais estável. (b) Segunda configuração de menor energia. Os números representam distâncias de ligação entre os átomos respectivos, em ångstroms. 
com respeito ao sítio substitucional na direção $\langle 111\rangle$, de aproximadamente $0.9 \AA$, ficando próximo do plano formado pelos três gálios primeiros vizinhos com os quais continua ligado, enquanto que o gálio ligado ao hidrogênio se desloca $0.17 \AA$ na direção contrária. Uma outra configuração similar a esta, foi obtida a partir da configuração inicial II, a qual apresenta uma energia total levemente maior $(0.07 \mathrm{eV})$. Na Figura 4.2(b) é mostrada a configuração que apresenta uma energia total muito próxima da configuração mais estável. Nesta também observamos a formação da ligação $N$-H, e neste caso de $1.04 \AA$. Aqui o deslocamento do nitrogênio é de $0.3 \AA$ e como no sistema anterior, não chega a ficar no mesmo plano dos gálios primeiros vizinhos. É importante notar que este sistema foi obtido a partir da configuração inicial VI da Figura 4.1, onde o hidrogênio centrado na ligação não ocupa a mesma ligação que o hidrogênio antiligante. Claramente esta configuração inicial é desfavorável energeticamente, provocando mudanças importantes na posição do hidrogênio uma vez que o sistema é permitido relaxar. Esta mesma configuração também foi obtida a partir da configuração inicial IV, mas com uma energia total $0.18 \mathrm{eV}$ maior.

Na Figura 4.3 são mostrados os gráficos de contorno da densidade de carga total para as duas configurações de menor energia. Aqui vemos que em ambas configurações é formada uma forte ligação $N$-H com caráter de dimero ligado fracamente à rede, apresentando o mesmo comportamento observado nas configurações de menor energia dos complexos envolvendo um hidrogênio $\left(N_{A s}-H\right)$, conforme mostradas na Figura 3.8. A configuração de menor energia, obtida a partir da configuração inicial I, Figura 4.3(a), mostra que o hidrogênio centrado na ligação se liga fracamente com nitrogênio, tendo uma interação maior com o gálio mais próximo. A distribuição de carga deste sistema apresenta uma certa similitude com o complexo $O_{A s}-H$, conforme mostrado na Figura 3.3(a), onde o dimero $N H$ pode ser substituido pelo átomo de oxigênio que tem a mesma valência. Assim podemos interpretar esta configuração da mesma forma que no complexo de oxigênio, onde o hidrogênio centrado na ligação atua como saturador de um dos gálios enquanto que o dimero $N H$ atua como um átomo altamente eletronegativo, ligando-se aos outros três gálios, formando uma hibridização tipo $s p^{2}$. Na Figura 4.3(b) mostramos a densidade de carga da configuração com a segunda menor energia total, obtida a partir da configuração VI da Figura 4.1. Aqui observamos que o hidrogênio centrado na ligação se aproxima do nitrogênio formando o dimero $N H$ e quebrando sua ligação com o gálio. Da mesma forma que no sistema anterior, esta configuração pode ser interpretada de forma similar à configuração AB-Ga do complexo de oxigênio cuja densidade de carga total, Figura 3.5(a), apresenta as mesmas características. O hidrogênio colocado na posição antiligante forma 

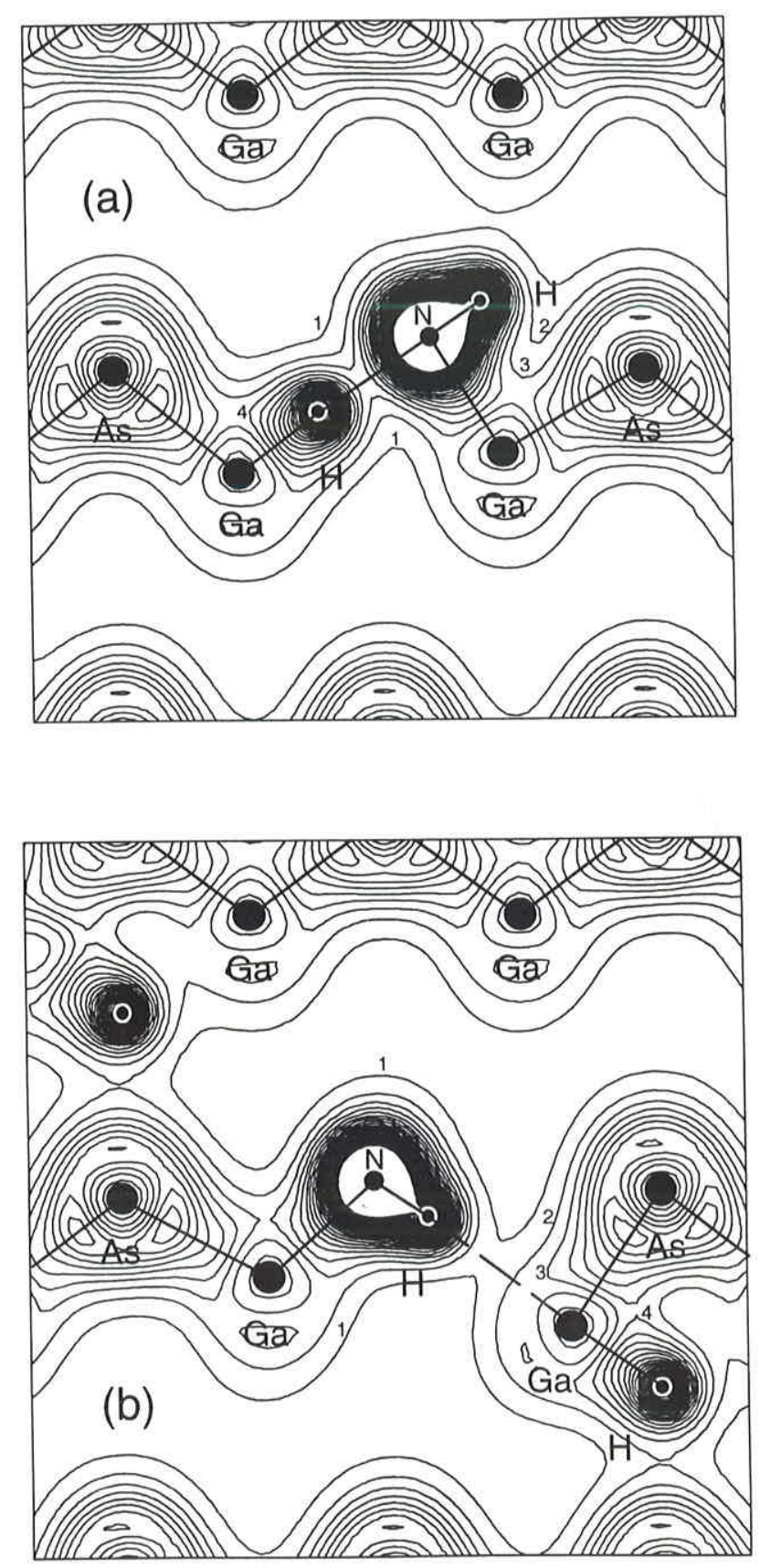

Figura 4.3: Gráficos de contorno da densidade de carga total de valência, através do plano [110], para as duas configurações de menor energia do complexo $\mathrm{N}_{\mathrm{As}}-\mathrm{H}_{2}$ em GaAs. (a) Sistema obtido a partir da configuração inicial I. (b) Sistema obtido a partir da configuração inicial VI. Unidades em $11.3 \mathrm{e} / \Omega$. 
uma ligação com o gálio atraindo carga e atuando como saturador, entretando o hidrogênio inicialmente centrado na ligação é atraido pelo nitrogênio formando o dimero $N H$, provocando a quebra da ligação $H-G a$.

A configuração obtida a partir dos sítios antiligantes para ambos os hidrogênios, configuração inicial $\mathrm{V}$, apresenta uma energia total $0.68 \mathrm{eV}$ maior com respeito ao sistema mais estável. A distância de ligação $N-H$ e $G a-H$ são encontradas como sendo de 1.05 e $1.62 \AA$, respectivamente. O nitrogênio se desloca do sítio substitucional na direção $\langle 111\rangle$ de $0.59 \AA$ ficando no mesmo plano dos três gálios mais próximos, entretanto o gálio ligado ao hidrogênio se desloca na direção $\langle\overline{1} \overline{1} \overline{1}\rangle$ de $0.89 \AA$, o que indica uma quebra de ligação $N$-Ga. Por último o sistema que apresenta a maior energia total entre as diferentes configurações estudadas tem cada um dos hidrogênios localizado em posições centradas na ligação, em duas das ligações de nitrogênio com gálio. Este sistema foi obtido a partir da configuração inicial III e apresenta uma energia total $0.84 \mathrm{eV}$ maior que a configuração mais estável. Os hidrogênios ficam mais próximos do nitrogênio que dos gálios, o que faz esperar uma ligação forte entre eles. As distâncias de ligação $N-H$ foram obtidas como sendo de $1.06 \AA$ para ambos casos com um ângulo $H-N-H$ de $74^{\circ}$. A incorporação dos hidrogênios nas ligações $N$-Ga provocam um deslocamento do nitrogênio de $0.66 \AA$ na direção $\langle 001\rangle$.

Na Figura 4.4 mostramos os gráficos de contorno da densidade de carga total para as configurações V e III. A configuração com os dois hidrogênios antiligantes, Figura 4.4(a), mostra claramente a tendência do hidrogênio de atuar como saturador quando se liga com um dos gálio e de formar um dimero quando se liga com o nitrogênio. Na Figura 4.4(b) mostramos a densidade de carga da configuração com os dois hidrogênios localizados em posições centradas na ligação (III), a qual apresenta a maior energia total entre os sistemas estudados. Aqui vemos a formação de uma molécula $\left(\mathrm{NH}_{2}\right)$, fracamente ligada à rede, a qual apresenta características semelhantes às impurezas substitucionais de oxigênio e nitrogênio estudadas no Capítulo 2. A formação deste sistema é originado na proximidade onde são deixados os átomos de hidrogênios com respeito ao nitrogênio e acreditamos que seja difícil de se formar naturalmente, considerando que a configuração inicial na qual é obtida apresenta os hidrogênio em uma situação bastante forçada.

Calculamos também a energia de ligação dos átomos de hidrogênio nas diferentes configurações do complexo $N_{A s}-H_{2}$ considerando a reação entre impurezas neutras dada por: $N_{A s}^{0}+2 H^{0} \rightarrow\left(N_{A s}-H_{2}\right)$. Esta reação, que levaria à formação do complexo, é a esperada em um regime tipo- $p$ do $G a A s$ onde o defeito $N_{A s}^{(0}$ apresenta a menor energia de formação, como mostra a 

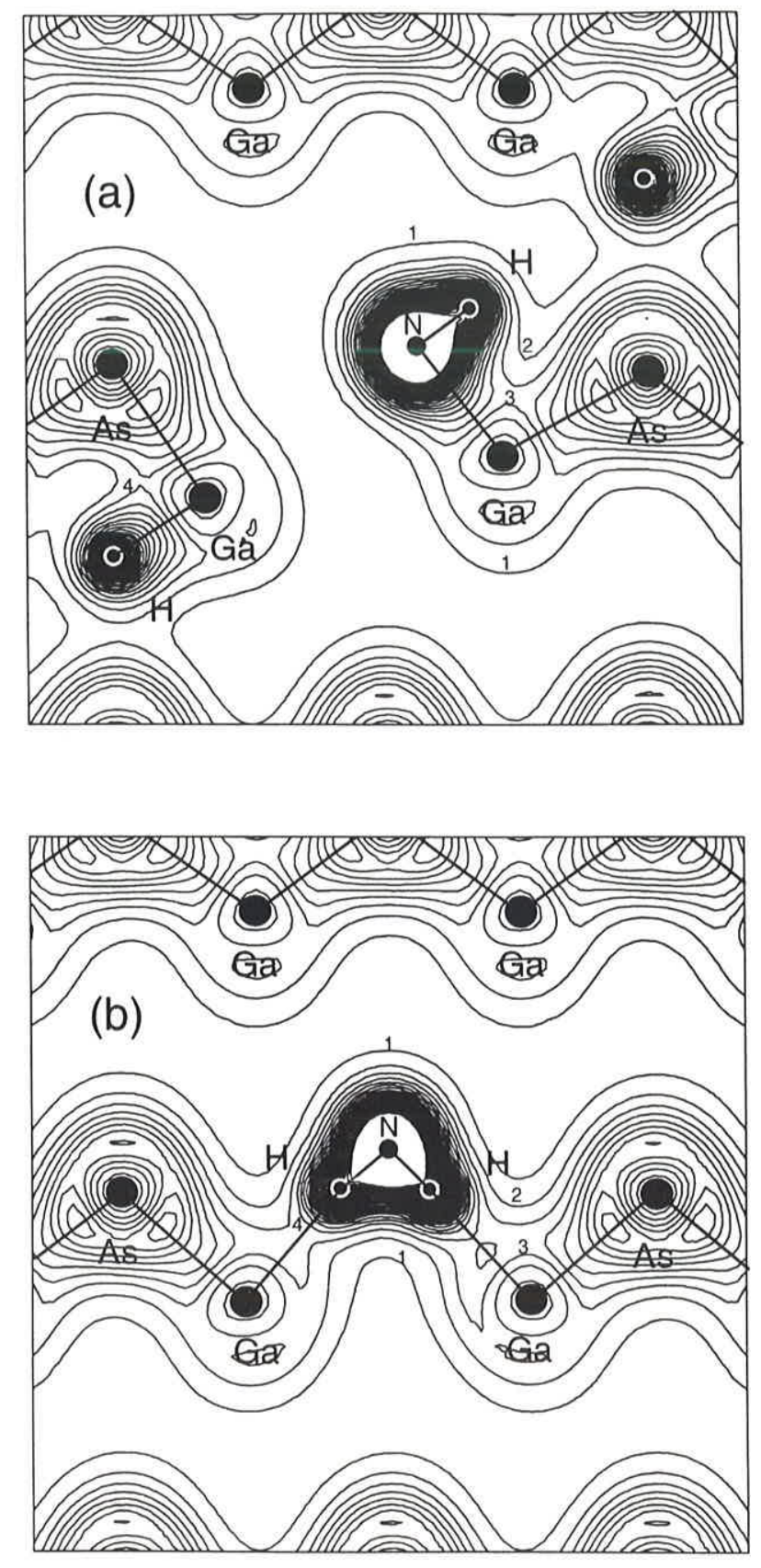

Figura 4.4: Gráficos de contorno da densidade de carga total de valência, através do plano [110], para duas configurações de equilíbrio metaestáveis do complexo $\mathrm{N}_{\mathrm{As}}-\mathrm{H}_{2}$ em GaAs. (a) Sistema obtido a partir da configuração inicial V. (b) Sistema obtido a partir da configuração inicial III. Unidades em $11.3 \mathrm{e} / \Omega$. 
Tabela 4.1: Geometria e energias do complexo $\mathrm{N}_{A s}-\mathrm{H}_{2}$ em GaAs para diferentes configurações de equilíbrio. $d_{B C}[N-H]\left(d_{B C}[H-G a]\right)$ é a distância interatômica $\mathrm{N}-\mathrm{H}$ (H-Ga) para o hidrogênio ocupando a posição centrada na ligação. $d_{A B}[N-H]\left(d_{A B}[H-G a]\right)$ é a distância interatômica $N-H$ (H-Ga) para o hidrogênio na posição antiligante. $\Delta E_{t}$ é a energia total relativa, com respeito à configuração mais estável, $e E_{b}$ a energia de ligação por átomo do hidrogênio. As distâncias estão em $\AA$ e as energias em eV.

\begin{tabular}{|c|c|c|c|c|c|c|}
\hline Config. & $d_{B C}[\mathrm{~N}-\mathrm{H}]$ & $d_{B C}[\mathrm{H}-\mathrm{Ga}]$ & $d_{A B}[\mathrm{~N}-\mathrm{H}]$ & $d_{A B}[\mathrm{H}-\mathrm{Ga}]$ & $\Delta E_{t}$ & $E_{b}$ \\
\hline \hline I & 1.95 & 1.54 & 1.05 & - & 0.00 & 1.99 \\
\hline II & 2.29 & 1.65 & 1.05 & - & 0.07 & 1.96 \\
\hline III & 1.06 & 2.01 & - & - & 0.84 & 1.57 \\
& 1.06 & 2.06 & & & & \\
\hline IV & 1.05 & 2.58 & - & 1.60 & 0.18 & 1.90 \\
\hline V & - & 3.91 & 1.05 & 1.62 & 0.68 & 1.65 \\
\hline VI & 1.04 & 3.10 & - & 1.62 & 0.04 & 1.97 \\
\hline
\end{tabular}

Figura 2.19. A energia de ligação dos hidrogênios foi calculada considerandose uma média entre as energias de ligação de cada hidrogênio em separado, que são desconhecidas, utilizando-se a seguinte relação:

$$
-E_{b}=\frac{1}{2}\left[E_{t}\left(N_{A s}-H_{2}\right)-E_{t}\left(N_{A s}^{0}\right)\right]+E_{t}\left(H_{T}^{0}\right)-E_{t}(G a A s),
$$

onde $E_{t}$ é a energia total dos respectivos sistemas e $E_{t}\left(H^{0}\right)$ a energia total do átomo de hidrogênio no sítio intersticial $\mathrm{T}$ em $G a A s$ que corresponde ao sistema de referência para o hidrogênio não ligado.

Nossos resultados para a energia de ligação nas diferentes configurações, conjuntamente com a energia total relativa e as distâncias entre os hidrogênios e seus vizinhos mais próximos são apresentados na Tabela 4.1. Aqui vemos que as energias totais com respeito à configuração de mínimo são menores que $0.85 \mathrm{eV}$, pequenas comparadas com as obtidas nos complexos $N_{A s}-H$, que são da ordem de $1.5 \mathrm{eV}$. Isto é devido ao tipo de ligação que apresentam os hidrogênios. Tipicamente um deles se liga ao nitrogênio formando o dimero $N H$ enquanto que o outro se liga a um gálio primeiro vizinho. Todas as configurações, com exceção da III, apresentam as mesmas ligações para os hidrogênios e portanto energias totais próximas. Com respeito à energia de 


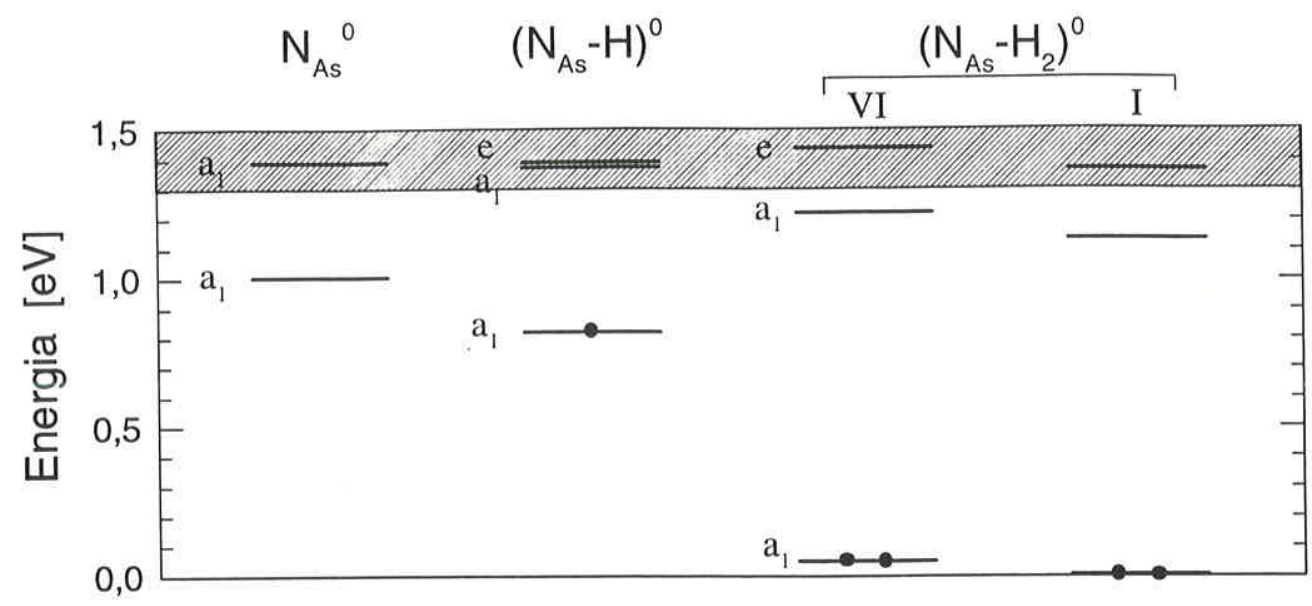

Figura 4.5: Representação esquemática dos autovalores de Kohn-Sham associados aos niveis de defeito no gap para o complexo $\mathrm{N}_{A s}-\mathrm{H}_{2}$ em GaAs, nas duas configurações de menor energia ( $\mathrm{l}$ e VI). Também são incluidos os niveis de defeito da impureza de nitrogênio $N_{A s}$ e o complexo $N_{A s}-H$. Os círculos cheios indicam a ocupação nos níveis e a região hachurada representa o fundo da banda de condução.

ligação vemos que para os hidrogênios fortemente ligados ao nitrogênio esta é da ordem de $1.6 \mathrm{eV}$ por átomo, essencialmente a mesma que no complexo $N_{A s}-H$ com o mesmo tipo de ligação $(\sim 1.7 \mathrm{eV})$. Isto nos pemite estimar a energia de ligação do hidrogênio ligado ao gálio nos complexos mais estáveis em $2.4 \mathrm{eV}$, já que conhecemos o valor médio nestas configurações, que é da ordem de $2.0 \mathrm{eV}$.

Nossos resultados para a estrutura eletrônica do complexo $\mathrm{N}_{A s}-\mathrm{H}_{2}$ nas duas configurações de menor energia, conjuntamente com os resultados obtidos para a impureza substitucional $N_{A s}^{0}$ e o complexo $N_{A s}-H$ em GaAs, são mostrados esquematicamente na Figura 4.5. Aqui vemos que o complexo envolvendo um hidrogênio, o qual é eletricamente ativo apresentando um nível semiocupado no meio da banda proibida, é passivado com a incorporação do segundo hidrogênio. O elétron adicionado por este hidrogênio preenche totalmente o nível do gap o qual se desloca para o topo da banda de valência. Entretanto um nível não degenerado desce do fundo da banda de condução se localizando a $1.14 \mathrm{eV}$ acima do topo da banda de valência. Comparando com a estrutura de níveis da impureza de nitrogênio isolada, vemos que estes sistemas apresentam mesma características de duplo aceitadores. É importante notar que as duas configurações mais estáveis, praticamente degeneradas em 
energia, apresentam a mesma estrutura eletrônica o que é uma conseqüência de formarem o mesmo tipo de ligação entre as impurezas de hidrogênio, nitrogênio e os átomos da rede. Finalmente verificamos que o complexo $\mathrm{N}_{A s}-\mathrm{H}_{2}$ em GaAs não possui níveis ocupados na banda proibida, sendo um centro aceitador eletricamente inativo. 


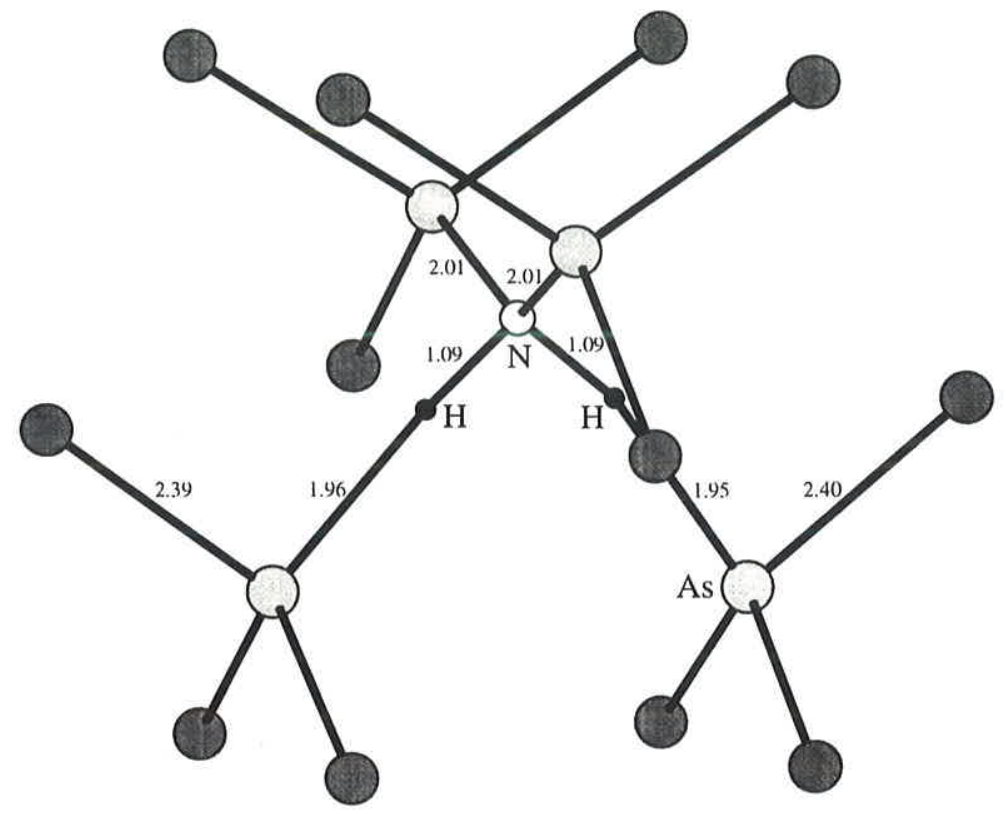

Figura 4.6: Configuração atômica de mínima energia obtida para o complexo $\mathrm{N}_{\mathrm{Ga}}-\mathrm{H}_{2}$ em GaAs. Os números representam distâncias de ligação entre os átomos respectivos, em ångstroms.

\subsection{Complexo $\mathrm{N}_{\mathrm{Ga}}-\mathrm{H}_{2}$ em $\mathrm{GaAs}$}

Nossos resultados para o complexo $\mathrm{N}_{G a}-\mathrm{H}_{2}$ em $\mathrm{GaAs}$ mostram que a configuração que apresenta a menor energia total tem ambos os hidrogênios localizados em posições centradas na ligação em simetria $C_{2 v}$, conservando a mesma distribuição atômica da configuração inicial, III na Figura 4.1. Os hidrogênios se ligam ao nitrogênio formando um ângulo $\mathrm{H}-\mathrm{N}-\mathrm{H}$ de $74^{\circ}$, onde os comprimentos de ligação $\mathrm{N}-\mathrm{H}$ e $H$ - $A s$ são aproximadamente os mesmos para ambos hidrogênios, 1.09 e $1.95 \AA$ respectivamente. A incoporação destes hidrogênios provoca um deslocamento do nitrogênio com respeito ao sítio substitucional em aproximadamente $0.8 \AA$ na direção $\langle 001\rangle$, enquanto que os arsênios ligados aos hidrogênios não sofrem deslocamentos consideráveis ficando nas suas posições originais. Na Figura 4.6 são mostradas as posições atômicas de equilíbrio e as distâncias de ligação mais importantes para este complexo na configuração de mínima energia. Esta mesma distribuição também foi obtida a partir da configuraçã̃o inicial II da Figura 4.1, onde os hidrogênios são deixados em posições puramente intersticiais sem interagir com o nitrogênio ou algum átomo da rede, 
com a energia total neste caso essencialmente a mesma à obtida na configuração III. A configuração com a segunda menor energia total, $0.73 \mathrm{eV}$ maior que III, apresenta a mesma distribuição atômica de mínima energia do complexo $N_{A s^{-}}$ $H_{2}$, mostrada na Figura 4.2(a), onde um dos hidrogênios ocupa a posição centrado na ligação $N$-As e o outro a posição antiligante com respeito ao nitrogênio. As distâncias de ligação $N-H$ e $H$-As são encontradas como sendo 1.70 e $1.53 \AA$, respectivamente, enquanto que a distância de ligação $N$ - $H$ para o hidrogênio antiligante é de $1.05 \AA$. Neste complexo o nitrogênio se desloca aproximadamente $0.9 \AA$ na direção $\langle 111\rangle$.

Na Figura 4.7 mostramos os gráficos de contorno da densidade de carga total para as duas configurações de menor energia. Aqui vemos que o sistema mais estável, Figura 4.7(a), mostra a formação da molécula $\mathrm{NH}_{2}$ da mesma forma que na configuração III do complexo $\mathrm{N}_{\mathrm{As}}-\mathrm{H}_{2}$, Figura 4.4(b), com o mesmo ângulo e comprimentos de ligação. A formação desta molécula é particularmente estável no complexo $\mathrm{NGa}_{\mathrm{G}}-\mathrm{H}_{2}$ devido às características do defeito substitucional. O nitrogênio substituindo um gálio adiciona dois elétrons ao sistema que "ocupam" dois orbitais flutuantes. A incorporação dos hidrogênios neste centro tendem a saturar estes orbitais devido a sua grande afinidade eletrônica, formando assim ligações fortes $\mathrm{N}-\mathrm{H}$. No caso do complexo $\mathrm{N}_{\mathrm{As}}-\mathrm{H}_{2}$, o nitrogênio é uma impureza substitucional isoeletrônica portanto sem orbitais flutuantes, logo a molécula $\mathrm{NH}_{2}$ é formada únicamente quando os hidrogênios são deixados suficientemente próximos do nitrogênio que, devido a sua alta eletronegatividade, consegue formar as ligações. Em outras circunstâncias um dos hidrogênios prefere se ligar a um átomo primeiro vizinho enquanto que o segundo ao nitrogênio formando o dimero $N H$. A Figura 4.7(b) mostra a densidade de carga total do complexo metaestável que apresenta a segunda menor energia total com respeito ao sistema estável $(0.73 \mathrm{eV})$, obtido a partir da configuração inicial I. Aqui vemos que um dos hidrogênios se liga ao nitrogênio formando o dimero $N H$ enquanto que o outro fica centrado na ligação $A s-N$, mais fortemente ligado ao arsênio. Acreditamos que a formação desta configuração seja influenciada pela metaestabilidade estrutural que apresenta a impureza de nitrogênio na direção $\langle 111\rangle$ a qual hospedaria ao dimero $N H$ da mesma forma como no complexo $N_{G a}-H$, Figura 3.12(b), entretando o segundo hidrogênio atuaria como saturador do orbital flutuante do arsênio. Esta distribuição de carga é similar à mostrada na Figura 4.3(a), correspondente à configuração de menor energia do complexo $N_{A s}-H_{2}$. Comparando as duas vemos que os hidrogênios ficam mais fortemente ligados quando os primeiros vizinhos são os arsênios, que é uma conseqüência da maior eletronegatividade deste átomo em relação ao gálio (2.2 e 1.8 respectivamente). 

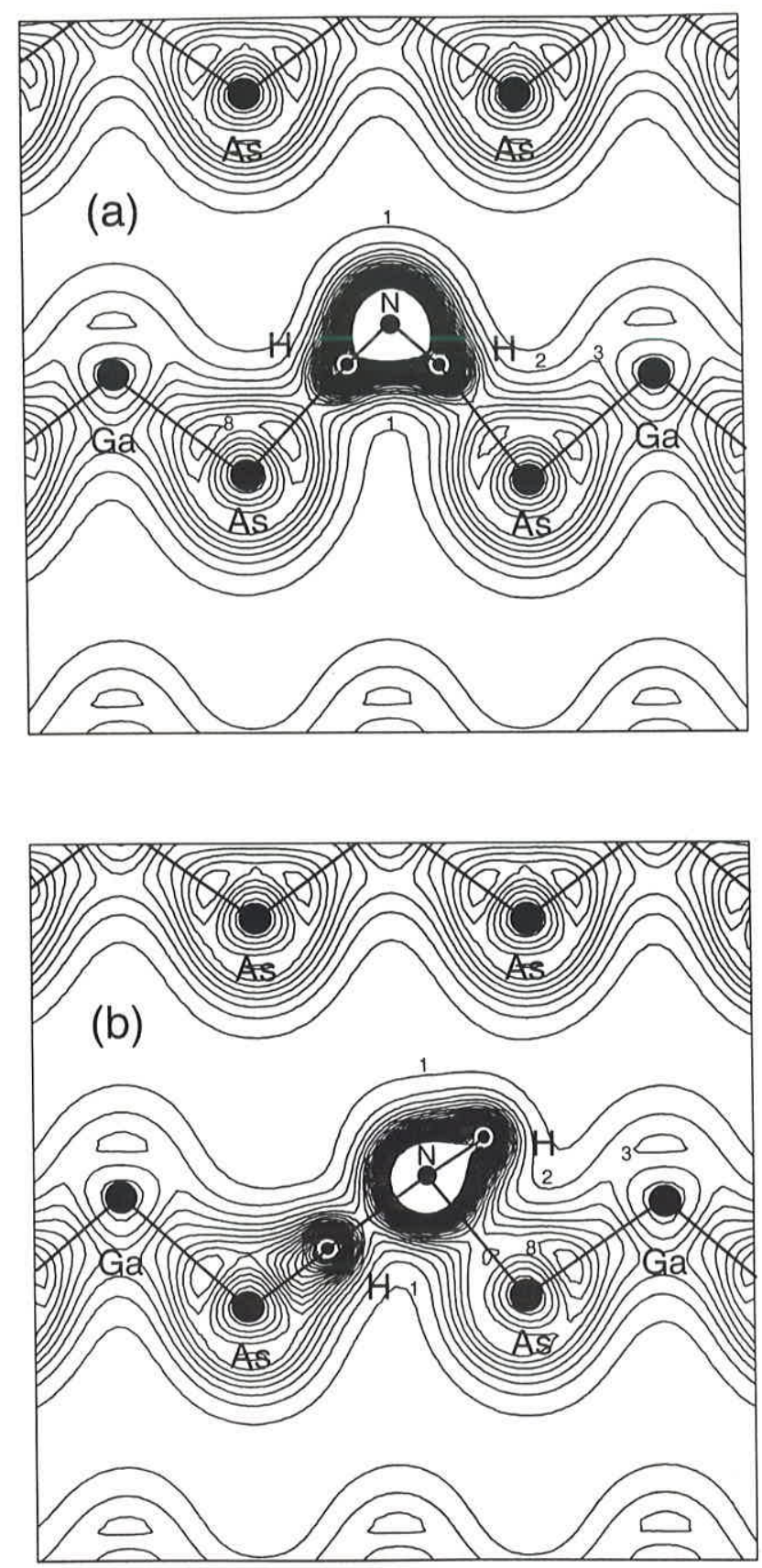

Figura 4.7: Gráficos de contorno da densidade de carga total de valência, através do plano [110], para as duas configurações de menor energia do complexo $\mathrm{N}_{\mathrm{Ga}}-\mathrm{H}_{2}$ em GaAs. (a) Sistema obtido a partir da configuração inicial III. (b) Sistema obtido a partir da configuração inicial I. Unidades em $11.3 \mathrm{e} / \Omega$. 
Na Figura 4.8 mostramos as densidades de carga total de valência dos complexos obtidos a partir das configurações e VI e IV que apresentam respectivamente energias totais de 0.89 e $1.02 \mathrm{eV}$ maiores que a energia total da configuração mais estável. Aqui vemos que estes sistemas apresentam distribuições atômicas muito próximas das suas respectivas configurações iniciais, mostrando uma maior estabilidade em relação às configurações equivalentes do complexo $N_{A s}-H_{2}$. A configuração VI, Figura 4.8(a), mostra a quebra da ligação $N$-As devida a formação da ligação do hidrogênio antiligante com o arsênio. O hidrogênio centrado na ligação forma o dimero $N H$ ligado a três arsênios. A configuração IV, Figura 4.8(b) mostra as mesmas características que o sistema anterior onde também é observado a quebra da ligação $N$-As provocada pela formação da ligação $H$-As. O sistema que apresenta a maior energia total, $1.39 \mathrm{eV}$ maior que a configuração mais estável e cuja densidade de carga não é mostrada aqui, apresenta uma distribuição atômica similar a sua configuração inicial (V). Nesta os dois hidrogênios antiligantes se ligam a seus respectivos vizinhos, formando o dimero $N H$ e a ligação $H$ - $A s$, onde a ligação $A s-N$ é quebrada. Uma característica comum observada nas configurações metaestáveis do complexo $\mathrm{N}_{\mathrm{Ga}}-\mathrm{H}_{2}$ é a formação de duas ligações fortes distribuidas em diferentes posições, uma delas é o dimero $N H$ ligado à rede e cujo comprimento de ligação da ordem de $1.05 \AA$ e a outra é a ligação $H$-As de comprimento $1.55 \AA$. Nesta última o hidrogênio se liga fortemente, atraindo carga da ligação de arsênio com nitrogênio ou o dimero, o que provoca a quebra da ligação. Esta situação também é observada no complexo $N_{A s}-H_{2}$ com maior intensidade ainda devido ao nitrogênio ser uma impureza substitucional isoeletrônica.

A energia de ligação para os átomos de hidrogênio nas diferentes configurações do complexo $\mathrm{N}_{G a}-\mathrm{H}_{2}$ foi calculada considerando a reação entre impurezas neutras dada por: $N_{G a}^{(0}+2 H^{(0)} \rightarrow\left(N_{G a}-H_{2}\right)$. Esta reação é esperada em um regime tipo- $p$ do material onde o nitrogênio substituindo um gálio, no estado de carga neutro, é o sistema que apresenta a menor energia de formação dentre outros estados de carga do mesmo centro. A energia de ligação dos hidrogênios foi calculada usando a equação (4.1), agora considerando a impureza $N_{G a}$ ao invés de $N_{A s}$. Como mencionamos anteriormente, esta energia de ligação corresponde a uma média entre dois tipos de ligações que os hidrogênios podem formar com nitrogênio ou um átomo da rede, mas no caso da configuração mais estável ambos hidrogênios formam ligações equivalentes, logo, a energia de ligação obtida a partir da equação (4.1) é igual para os dois hidrogênios. 

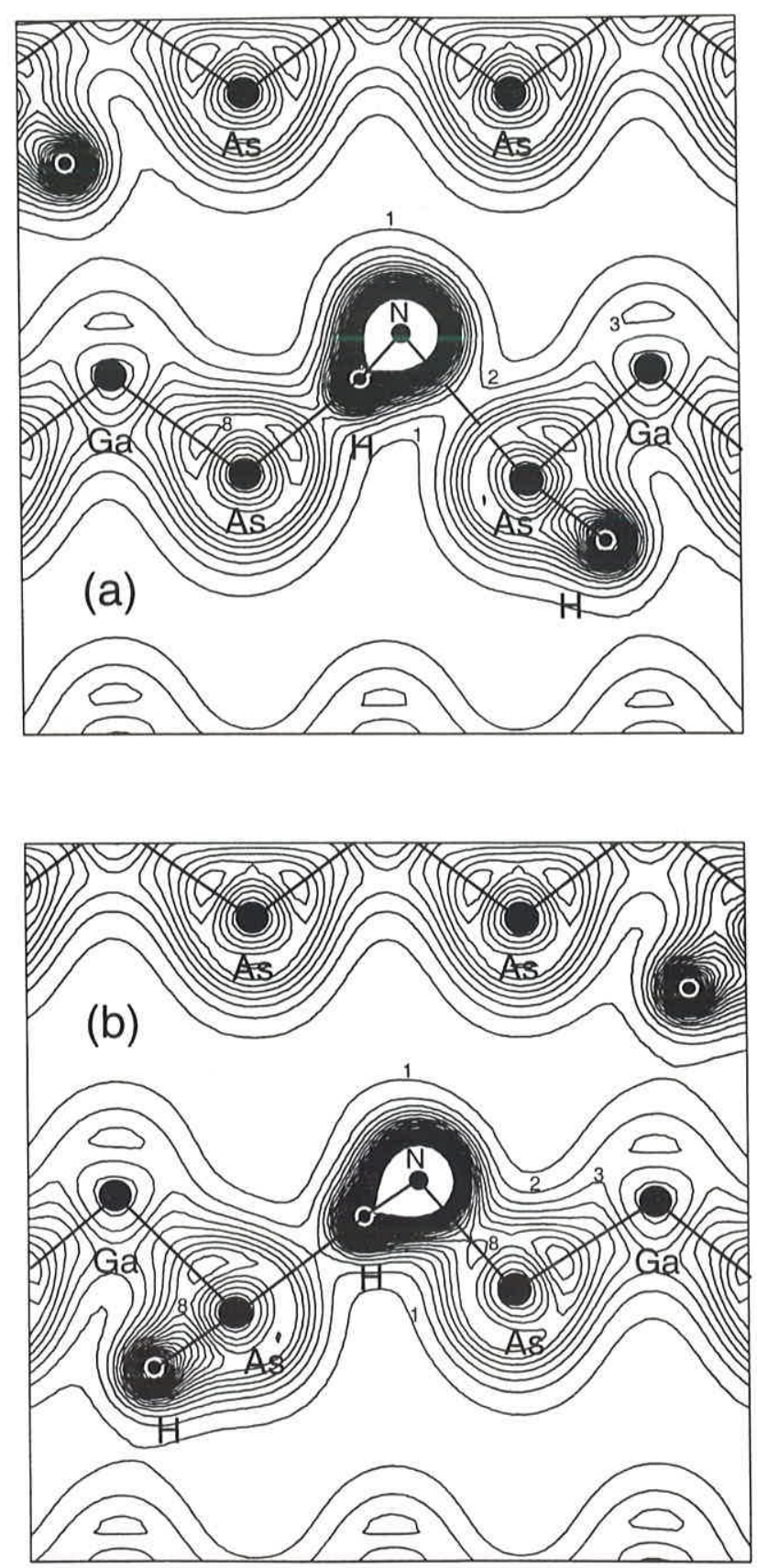

Figura 4.8: Gráficos de contorno da densidade de carga total de valência, através do plano [110], para duas configurações de equilíbrio metaestáveis do complexo $\mathrm{N}_{\mathrm{Ga}}-\mathrm{H}_{2}$ em GaAs. (a) Sistema obtido a partir da configuração inicial VI. (b) Sistema obtido a partir da configuração inicial IV. Unidades em $11.3 \mathrm{e} / \Omega$. 
Tabela 4.2: Geometria e energias do complexo $\mathrm{N}_{G a}-\mathrm{H}_{2}$ em GaAs para diferentes configurações de equilíbrio. $d_{B C}[N-H]\left(d_{B C}[H-A s]\right)$ é a distância interatômica $N$-H (H-As) para o hidrogênio ocupando a posição centrada na ligação. $d_{A B}[N-H]\left(d_{A B}[H-A s]\right)$ é a distância interatômica $N-H$ (H-As) para o hidrogênio na posição antiligante. $\Delta E_{t}$ é a energia total relativa, com respeito à configuração mais estável, e $E_{b}$ a energia de ligação por átomo de hidrogênio. As distâncias estão em $\AA$ e as energias em $\mathrm{eV}$.

\begin{tabular}{|c|c|c|c|c|c|c|}
\hline Config. & $d_{B C}[\mathrm{~N}-\mathrm{H}]$ & $d_{B C}[\mathrm{H}-\mathrm{As}]$ & $d_{A B}[\mathrm{~N}-\mathrm{H}]$ & $d_{A B}[\mathrm{H}-\mathrm{As}]$ & $\Delta E_{t}$ & $E_{b}$ \\
\hline \hline I & 1.70 & 1.53 & 1.05 & - & 0.73 & 2.51 \\
\hline II & 1.10 & 1.97 & - & - & 0.04 & 2.85 \\
& 1.09 & 1.97 & - & - & & \\
\hline III & 1.09 & 1.95 & - & - & 0.00 & 2.88 \\
& 1.09 & 1.96 & & & & \\
\hline IV & 1.06 & 2.23 & - & 1.54 & 1.02 & 2.37 \\
\hline V & - & - & 1.05 & 1.55 & 1.39 & 2.18 \\
\hline VI & 1.07 & 2.02 & - & 1.57 & 0.89 & 2.43 \\
\hline
\end{tabular}

Na Tabela 4.2 são mostrados os valores da energia total com respeito da configuração mais estável, as distâncias entre os átomos de hidrogênio e seus vizinhos mais próximos e a energia de ligação. Aqui notamos que a energia de ligação dos hidrogênios, na configuração de mínima energia (III), é da ordem de $0.9 \mathrm{eV}$ maior que seu equivalente no complexo $\mathrm{N}_{A_{s}}-\mathrm{H}_{2}$ (configuração I), demostrando que as impurezas substitucionais que apresentam ligações flutuantes conseguem se ligar mais fortemente aos hidrogênios quando formam complexos.

Nossos resultados para estrutura eletrônica do complexo $\mathrm{NGa}_{\mathrm{Ga}}-\mathrm{H}_{2}$ na configuração mais estável (III) mostra que este centro é eletricamente inativo apresentando três níveis não degenerados na banda proibida, dois níveis totalmente ocupados localizados a 0.05 e $0.17 \mathrm{eV}$ e um vazio a $0.76 \mathrm{eV}$, acima do topo da banda de valência. A configuração com a segunda menor energia total (I) mostra uma estrutura eletrônica diferente, onde são encontrados três níveis não degenerados na banda proibida, um totalmente ocupado localizado a $0.25 \mathrm{eV}$ e dois vazios a 1.15 e $1.25 \mathrm{eV}$, acima do topo da banda de valência. Na Figura 4.8 é mostrada uma representação esquemática dos níveis destas configurações juntamente com a estrutura eletrônica do defeito substitucional $N_{G a}^{0}$ e o complexo $N_{G a}-H$. Os níveis da configuração I representam 


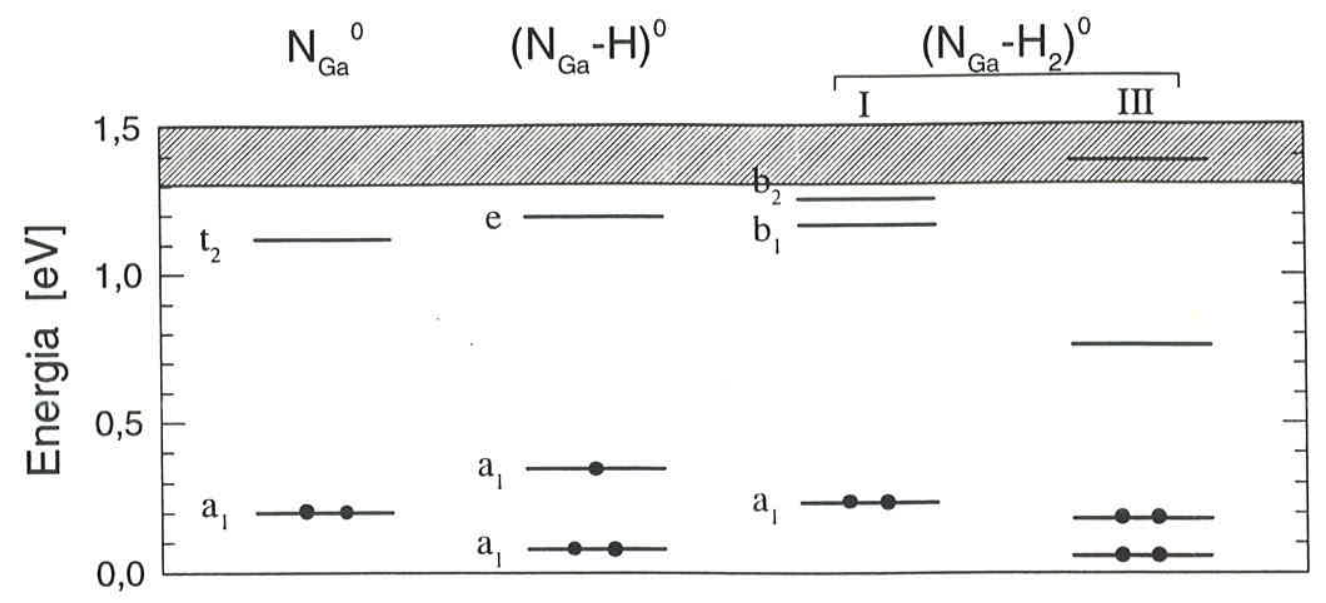

Figura 4.9: Representação esquemática dos autovalores de Kohn-Sham associados aos niveis de defeito do gap para o complexo $\mathrm{N}_{\mathrm{Ga}}-\mathrm{H}_{2}$ em GaAs, nas duas configurações de menor energia (III e I). Também são incluidos os níveis de defeito da impureza de nitrogênio $N_{G a}$ e o complexo $N_{G a}-H$. Os círculos cheios indicam a ocupação nos níveis e a região hachurada representa o fundo da banda de condução.

uma situação onde um segundo hidrogênio é incorporado ao complexo neutro $N_{G a}-H$ cujo elétron ocupa totalmente o nível $a_{1}$ semi-ocupado, deslocando-se levemente em direção da banda de valência, neutralizando sua atividade, enquanto que o nível $a_{1}$, totalmente ocupado, entra na banda de valência. Esta configuração mostra uma estrutura de níveis semelhante ao defeito $N_{G a}^{0}$. A configuração III representa uma situação onde os dois hidrogênios são incorporados simultâneamente ao defeito $N_{G a}^{0}$ formando um sistema molecular. Sua estrutura de níveis pode ser interpretada como um defeito substitucional de valência sete substituindo um átomo de valência três, portanto adicionando quatro elétrons ao sistema.

Neste capítulo estudamos a formação de complexos envolvendo a impureza substitucional de nitrogênio e dois hidrogênios intersticiais. De acordo com nossos cálculos da energia de formação para o defeito substitucional, sabemos que nitrogênio substituindo um arsênio no estado de carga neutro é o defeito mais favorável de se formar em um regime tipo- $p$ do material, logo é esperado que em um processo de hidrogenação sejam formados complexos envolvendo a impureza susbtitucional $N_{A s}^{0}$ e átomos de hidrogênio, considerando-se a grande eletronegatividade do nitrogênio. Estudos recentes de modos locais de vibração no infravermelho têm atribuidos três modos vibracionais ao complexo $N_{A s}-H_{2}$ em $G a P$ e $G a A s,{ }^{15.16}$ o qual teria um dos hidrogênios na posição cen- 
trada na ligação e o outro antiligante em torno do nitrogênio. Nossos resultados para o complexo $\mathrm{N}_{A s}-\mathrm{H}_{2}$ em GaAs mostram que a configuração proposta pelos experimentais é efectivamente aquela que apresenta a menor energia para este sistema. Uma segunda configuração com uma energia total levemente superior (0.04 eV) também é encontrada para o complexo. Nesta um dos hidrogênios ocupa uma posição centrada na ligação e o outro antiligante com respeito a um dos gálio, também em simetria trigonal. Nestas duas configurações um dos hidrogênios se liga fortemente com o nitrogênio apresentando características de dimero, enquanto que o segundo hidrogênio prefere se ligar ao gálio. A energia de ligação para os hidrogênios ligados ao nitrogênio e gálio é calculada em $1.6 \mathrm{eV}$ e $2.4 \mathrm{eV}$ respectivamente. Nestes cálculos também verificamos que este centro não apresenta níveis profundos no gap. Encontramos um único nível vazio não degenerado localizado aproximadamente a $1.2 \mathrm{eV}$ acima do topo da banda de valência, o qual é visto nas duas configurações de mínima energia.

Nossos resultados para o complexo $\mathrm{NGa}_{\mathrm{Ga}}-\mathrm{H}_{2}$, mostram que a configuração que apresenta a menor energia tem os dois hidrogênios fortemente ligados ao nitrogênio em posições centradas na ligação, formando uma estrutura molecular do tipo $\mathrm{NH}_{2}$ fracamente ligada à rede. Esta estrutura foi obtida a partir de duas configurações onde ambos os hidrogênios foram colocados em sítios simétricos próximos do nitrogênio. A energia de ligação para estes foi calculada em $2.88 \mathrm{eV}$. A segunda configuração de menor energia, $0.73 \mathrm{eV}$ maior que a configuração anterior, tem um dos hidrogênios em uma posição antiligante com respeito ao nitrogênio e o outro centrado na ligação. O hidrogênio antiligante forma a estrutura de dimero com o nitrogênio enquanto que o segundo hidrogênio se liga fortemente ao arsênio. As energias de ligação são calculadas em 2.9 e $2.1 \mathrm{eV}$ respectivamente. Acreditamos que a estrutura molecular da configuração de menor energia seja uma situação difícil de se formar naturalmente, considerando a simetria das posições iniciais e sua proximidade com nitrogênio. Como vimos neste complexo, os arsênios primeiros vizinhos conseguem se ligar fortemente aos hidrogênios o que os impediria de chegar perto do nitrogênio simultaneamente, entretanto uma situação onde um dos hidrogênios forma uma ligação com o nitrogênio e posteriormente outro hidrogênio e ligado pelos arsênios primeiros vizinhos parece ser uma situação mais realista. Com respeito a estrutura eletrônica, nossos resultados mostram que este complexo é eletricamente inativo mas apresentando níveis não degenerados totalmente ocupados e vazios na banda proibida. As duas configurações de menor energia mostram que este complexo é um duplo aceitador. 


\section{Capítulo 5}

\section{Conclusões}

Neste trabalho estudamos as propriedades microscópicas de defeitos simples e complexos envolvendo os átomos leves de oxigênio, nitrogênio e hidrogênio no cristal semicondutor de arseneto de gálio ( $G a A s)$, a partir de métodos teóricos de primeiros princípios. Consideramos as impurezas isoladas de oxigênio substituindo um arsênio $\left(O_{A s}\right)$, nitrogênio substituindo um arsênio $\left(N_{A s}\right)$ e nitrogênio substituindo um gálio $\left(N_{G a}\right)$. Nestes sistemas foram determinadas as configurações atômicas de equilíbrio para cinco estados de carga, e foram calculadas as respectivas energias de formação e estrutura eletrônica. Consideramos ainda estes mesmos defeitos substitucionais interagindo com um átomo de hidrogênio intersticial, de modo a formar o complexos neutros $O_{A s}-H$, $N_{A s}-H$ e $N_{G a}-H$. Foram examinadas diferentes posições de alta simetria para o átomo de hidrogênio afim de determinar a configuração de menor energia. Nestes cálculos foram estudados a estabilidade dos complexos e o fenômeno de passivação nos centros eletricamente ativos como também a ativação de centros não ativos, provocados com a incorporação do hidrogênio nos defeitos substitucionais. Consideramos também a incorporação de dois hidrogênios nos defeitos substitucionais envolvendo nitrogênio, $\mathrm{N}_{\mathrm{As}}-\mathrm{H}_{2}$ e $\mathrm{N}_{\mathrm{Ga}}-\mathrm{H}_{2}$. A configuração de menor energia para estes complexos também foi determinada analisando diferentes sítios intersticiais de alta simetria para ambos hidrogênios. Nestes cálculos foram estudados a estabilidade dos complexos como também suas estruturas eletrônicas afim de verificar as modificações sofridas pelos centros passivados ou ativados com a incorporação do segundo átomo de hidrogênio.

Nossos resultados para o centro $O_{A s}$ em GaAs apontam a um defeito profundo que em seu estado de carga neutro introduz um nível de impureza não degenerado, aproximadamente no meio da banda proibida, o qual é ocupado com um elétron. Um segundo nível tridegenerado também associado à im- 
pureza é observado resonante no fundo da banda de condução. Nossos cálculos da energia de formação mostram que este centro pode existir em cinco diferentes estados de carga, de positivo a três vezes negativo, onde o estado de carga 2- é instável, e portanto apresentando um comportamento U-negativo. ${ }^{41}$ Nos estados de carga positivo, neutro e negativo não são observados deslocamentos do átomo de oxigênio com respeito ao sítio substitucional, entretanto o estado 2- mostra um deslocamento de aproximadamente $0.7 \AA$ na direção $\langle 111\rangle$, o que propomos como sendo atribuído a uma distorção Jahn-Teller ${ }^{47}$ devido ao último nível ocupado, neste estado de carga, ser tridegenerado. Também é observado um sítio metaestável a aproximadamente $0.8 \AA$ na direção $\langle 100\rangle$. O defeito no estado de carga 3- mostra um único deslocamento de $0.8 \AA$ na direção $\langle 100\rangle$. Para os sistemas com configurações de equilíbrio em simetria $C_{2 v}$ é observada a formação das ligações $G a-O-G a$ e $G a-G a$ entre os primeiros vizinhos e a impureza. Comparando com resultados experimentais notamos que existem dois pontos concordantes com nossos cálculos, estes são a geometria de defeito de simetria $C_{2 v}$ e o comportamento U-negativo, entretanto o ponto discordante é o estado de carga onde estes fenômenos são observados. Experimentalmente a distorção da impureza de oxigênio é atribuida a três estados de carga $(0,1 \text { - e 2- })^{40}$ e o caráter U-negativo ao estado de carga negativo. ${ }^{42.43}$ Para explicar as distorções foi invocado o efeito pseudo-Jahn-Teller ${ }^{48,49}$ da mesma forma que no centro-A em silício. ${ }^{5}$ Nossos resultados mostram que o defeito no estado de carga neutro e negativo não apresenta distorções, entretanto nos estados 2- e 3- este é encontrado nas simetrias $C_{3 v}$ e $C_{2 v}$, respectivamente, onde o estado instável que exibe o comportamento U-negativo é o duplamente negativo. As distorções por nos obtidas são claramente explicadas em termos do efeito Jahn-Teller. Como vemos ambos os resultados podem ser igualmente justificados, entretanto acreditamos que estudos experimentais mais apurados poderiam resolver esta questão.

O defeito $N_{A s}$ em GaAs é um centro isoeletrônico que apresenta um nível de defeito não degenerado e vazio na banda proibida, localizado aproximadamente a $1.0 \mathrm{eV}$ acima do topo da banda de valência. Um segundo nível de defeito, também não degenerado, é observado resonante no fundo da banda de condução. Nossos cálculos para a energia de formação mostram que este centro pode existir em quatro estados de carga, desde neutro até triplamente negativo. Não foram observadas distorções da impureza de nitrogênio, que conserva sua simetria tetraédrica nos cinco estados de carga estudados. As ligações para este sistema são produto de uma hibridização $s p^{3}$. Até a presente data não foram comunicados trabalhos experimentais deste centro em GaAs. 
O defeito $N_{G a}$ em $G a A s$ no estado de carga neutro mostra dois níveis de defeito na banda proibida, um não degenerado totalmente ocupado e um triplamente degenerado vazio, localizados aproximadamente a 0.2 e $1.1 \mathrm{eV}$ acima do topo da banda de valência, respectivamente. Neste estado de carga a impureza não sofre distorção com respeito ao sítio substitucional, entretanto se ela é deslocada na direção $\langle 111\rangle(\sim 1.1 \AA)$ é observada uma metaestabilidade estrutural a qual também tem sido observada teórica e experimentalmente em outras impurezas do tipo antisítio de arsênio em GaAs. ${ }^{61}$ A energia total no sítio metaestável é $0.32 \mathrm{eV}$ maior que no sítio substitucional onde a barreira de energia é calculada em $0.44 \mathrm{eV}$. No estado de carga negativo, o defeito sofre um deslocamento também na direção $\langle 111\rangle$, que atribuimos a uma distorção JahnTeller. Nossos cálculos da energia de formação mostram que este defeito pode existir em quatro estados de carga, de neutro até triplamente negativo, onde o estado negativo é instável, i.e., o defeito exibe um comportamento U-negativo. $\mathrm{O}$ fato deste centro apresentar uma metaestabilidade estrutural mostra que as ligações entre o nitrogênio e os arsênios primeiros vizinhos podem se formar a partir de hibridizações $s p^{3}$ e $s p^{2}$ com relativa facilidade. Comparando as energias de formação entre os dois tipos de impurezas substitucionais de nitrogênio $\left(N_{A s}\right.$ e $\left.N_{G a}\right)$, notamos que para um regime tipo- $p$ do material, o defeito $N_{A s}^{0}$ é o mais favorável de se formar tanto em um ambiente rico em átomos de gálio como de arsênio entretanto, para um regime tipo- $n$, a formação dos defeitos $N_{A s}^{3-}$ e $N_{G a}^{3-}$ ocorre como processos competitivos igualmente prováveis.

Nossos resultados para o complexo neutro formado pela impureza substitucional de oxigênio e um hidrogênio intersticial $\left(O_{A s}-H\right)$, mostram que o hidrogênio neste complexo atua como saturador de um orbital flutuante do gálio, interagindo fracamenta com o átomo de oxigênio. Entretanto o oxigênio prefere se ligar mais fortemente aos outros três gálios primeiros vizinhos em uma hibridização tipo $s p^{2}$. A incorporação do hidrogênio elimina a atividade elétrica do centro $O_{A s}^{0}$ onde também são removidos os níveis ocupados da banda proibida. É importante salientar que a remoção dos níveis ocupados do gap somente ocorre para os complexos que apresentam configurações como as acima mencionadas. No caso do complexo $N_{A s}-H$, o átomo de hidrogênio se liga fortemente ao nitrogênio formando um dimero $N H$ o qual fica ligado à rede sem romper nenhuma ligação, em uma hibridização tipo $s p^{3}$. A incorporação do hidrogênio no defeito $N_{A s}^{()}$provoca sua ativação elétrica, onde este complexo apresenta um nível de defeito semiocupado aproximadamente no meio do gap. Se consideramos o dimero $N H$ como uma impureza substitucional, cuja valência é seis, vemos que esta mostra as mesmas características que o átomo de oxigênio no defeito $O_{A s}^{()}$, apresentando a mesma estrutura eletrônica. 
Isto confirma a preferência da impureza de oxigênio neutra a se hibridizar tipo $s p^{3}$ quando se liga aos quatro gálios primeiros vizinhos. Para o complexo $N_{G a^{-}}$ $H$ também é observado a formação do dimero $N H$ na mesma configuração do caso anterior. Neste caso $N H$ se liga fortemente a três dos arsênios primeiros vizinhos ficando aproximadamente no mesmo plano, enquanto que a ligação com o quarto arsênio é mais fraca. Uma outra configuração similar a esta, com uma energia total da ordem de $0.3 \mathrm{eV}$ maior que a configuração mais estável, mostra a quebra de uma das ligações do dimero com um arsênio, ficando este com coordenação três. Estas duas configurações mostram que a metaestabilidade observada no defeito $N_{G a}^{0}$, que favorece uma hibridização do tipo $s p^{2}$, influencia a formação do complexo. A incorporação do hidrogênio no defeito substitucional também provoca sua ativação elétrica, onde a estrutura eletrônica do complexo apresenta um nível de defeito semiocupado na parte inferior do gap. Os três complexos estudados $\left(O_{A s}-H, N_{A s}-H\right.$ e $\left.N_{G a}-H\right)$ mostram que o átomo de hidrogênio pode neutralizar a atividade elétrica ou ativa-la em caso desta não existir, em defeitos envolvendo impurezas substitucionais que introduzem níveis profundos na banda proibida do $G a A s$. No caso do complexo $O_{A s}-H$ o oxigênio prefere se ligar a três gálios ao invés de formar o dimero $O H$, deixando ao hidrogênio a função de saturador. Este comportamento sugere que o hidrogênio atuaria como catalizador em um processo de difução do oxigênio substitucional. Nos complexos $N_{A s}-H$ e $N_{G a}-H$ o nitrogênio forma uma ligação forte com o hidrogênio apresentando um carácter molecular. Estes comportamentos diferentes para átomos com similares características poderiam ser explicados a partir de um ponto de vista puramente atômico, considerando o número de elétrons no orbital $p$ dos átomos de oxigênio e nitrogênio em uma configuração de baixo spin. Neste caso o hidrogênio preferiria se ligar ao nitrogênio de modo a reduzir o spin do sistema formando o dimero $\mathrm{NH}$, entretanto a formação do dimero $O H$ no caso do oxigênio produziria uma configuração de alto spin que é desfavorável.

Nossos resultados para o complexo formado pelo nitrogênio substituindo um arsênio e dois hidrogênios intersticiais $\left(N_{A s}-H_{2}\right)$, mostram um dos hidrogênios ligado fortemente ao nitrogênio em posição antiligante, formando o dímero $N H$, e o segundo hidrogênio centrado na ligação mais fortemente ligado ao gálio, i.e., formando um complexo do tipo $N_{A s}-H_{2}^{*}$. Uma segunda configuração apresentando aproximadamente a mesma energia que a anterior, tem um dos hidrogênios formando o dímero $N H$ agora em posição centrada na ligação e o segundo hidrogênio ligado ao gálio em posição antiligante. Estas duas configurações apresentam simetria $C_{3 v}$. Experiencias recentes de espectroscopia de modos locais de vibração ${ }^{16}$ têm identificado a configuração $N_{A s}-H_{2}^{*}$ 
como a mais estável, em concordância com nossos cálculos. Nesta configuração o dimero $\mathrm{NH}$ fica ligado aos três gálios primeiros vizinhos enquanto que o segundo hidrogênio atua como saturador do orbital flutuante do quarto gálio, mostrando as mesmas características que o complexo $O_{A s}-H$. A analogia entre estes dois sistemas confirma o carácter atômico do dimero $N H$ (com a mesma valência do oxigênio) e a preferência para formar hibridização do tipo $s p^{2}$. A estrutura eletrônica do complexo $N_{A s}-H_{2}$ mostra que este defeito é eletricamente inativo não apresentando níveis ocupados no gap. Devido ao dímero $\mathrm{NH}$ se formar nos complexos com um e dois hidrogênios, podemos considerar a formação do complexo $\mathrm{N}_{\mathrm{As}^{-}} \mathrm{H}_{2}$ como originado na incorporação de um segundo hidrogênio no complexo $N_{A s}-H$, o qual é eletricamente ativo. Desta forma, a estrutura eletrônica do complexo envolvendo dois hidrogênios pode ser explicada como a passivação do complexo ativo $N_{A s}-H$, pela incorporação do segundo hidrogênio. Estes resultados mostram que a atividade elétrica de um defeito pode ser eliminada e posteriormente ativada por sucessivas incorporações de átomos de hidrogênio ao defeito original.

Finalmente nossos resultados para o complexo $\mathrm{N}_{G a}-\mathrm{H}_{2}$ mostram a formação de uma estrutura com características de molécula do tipo $\mathrm{NH}_{2}$ com os dois hidrogênios em posições centradas na ligação, fortemente ligados ao nitrogênio e fracamente ligados a rede. Uma segunda configuração com energia total de $0.73 \mathrm{eV}$ maior que o sistema anterior mostra uma configuração do tipo $N_{G a}-H_{2}^{*}$. Estes sistemas são eletricamente inativos, apresentando diferentes estruturas eletrônicas atribuidas aos diferentes tipos de ligações que formam. No caso da configuração molecular são observados dois níveis totalmente ocupados na parte inferior do gap e outro vazio no meio, todos não degenerados. Este níveis são atribuídos à "molécula" $\mathrm{NH}_{2}$ atuando como impureza substitucional. Entretanto o sistema $N_{G a}-H_{2}^{*}$ apresenta uma estrutura eletrônica onde é observado um único nivel totalmente ocupado na parte inferior do gap e dois níveis vazios na parte superior; sendo esta estrutura atribuida a um processo de passivação sofrido pelo complexo $N_{G a}-H$ com a incorporação de um segundo hidrogênio. Por último acreditamos que o complexo "molecular" seja um sistema pouco provável de se formar naturalmente devido às condições iniciais do sistema serem pouco representativas de uma situação real, isto é, as posições iniciais onde os hidrogênios são deixados antes de iniciar a relaxação, forçariam uma aproximação simetrica e simultânea dos hidrogênios ao nitrogênio. Uma situação mais realista seria a incorporação não simultânea dos hidrogênios, que levaria à uma configuração de equilíbrio do tipo $N_{G a}-H_{2}^{*}$, que apresenta a segunda menor energia total. 


\section{Apêndice A}

\section{Geração dos Pseudopotenciais}

Em cálculos de estrutura eletrônica onde as funções de onda são expandidas em um conjunto de ondas planas e os átomos são descritos por pseudopotenciais, o tamanho da base está diretamente relacionado com a profundidade dos orbitais atômicos na região de caroço. Um dos problemas mais sérios que enfrentamos neste tipo de cálculo, é a boa descrição dos orbitais $p$ nos elementos da primeira linha da Tabela Periódica e dos orbitais $d$ nos metais de transição. Estes orbitais são muito localizados na região de caroço de forma que uma descrição realística deles em um sistema cristalino, requer um grande número de ondas planas e, por conseqüência, um considerável esforço computacional. Nos nossos cálculos utilizamos o pseudopotencial ab initio, de norma conservada de Troullier- Martins, ${ }^{27}$ o qual foi construído especialmente para descrever de forma eficiente, átomos com orbitais fortemente localizados. A suavidade deste pseudopotencial reduz drasticamente o número de ondas planas necessárias para alcançar a convergência.

Todos os pseudopotenciais utilizados por nós, foram transformados a uma forma não local utilizando o procedimento de Kleinman-Bylander. ${ }^{24}$ $\mathrm{Na}$ identificação de possíveis estados "fantasmas" foi utilizado o teorema de Gonze et $a .^{25}$ e também calculamos explicitamente as derivadas logarítmicas das pseudofunções de onda radial, no intervalo de energia da banda de valência e fundo da banda de condução. Nos casos onde se verificou a aparição de estados "fantasmas", como por exemplo no orbital $d$ do gálio, estes foram removidos aumentando-se levemente o raio de corte para este orbital. Deve-se ter cuidado neste procedimento já que na medida que o raio de corte é aumentado, a transferibilidade do pseudopotencial para o orbital em questão vê-se comprometida. A transferibilidade é a característica de reproduzir as propriedades atômicas, descritas pelos pseudopotenciais, quando transportados 

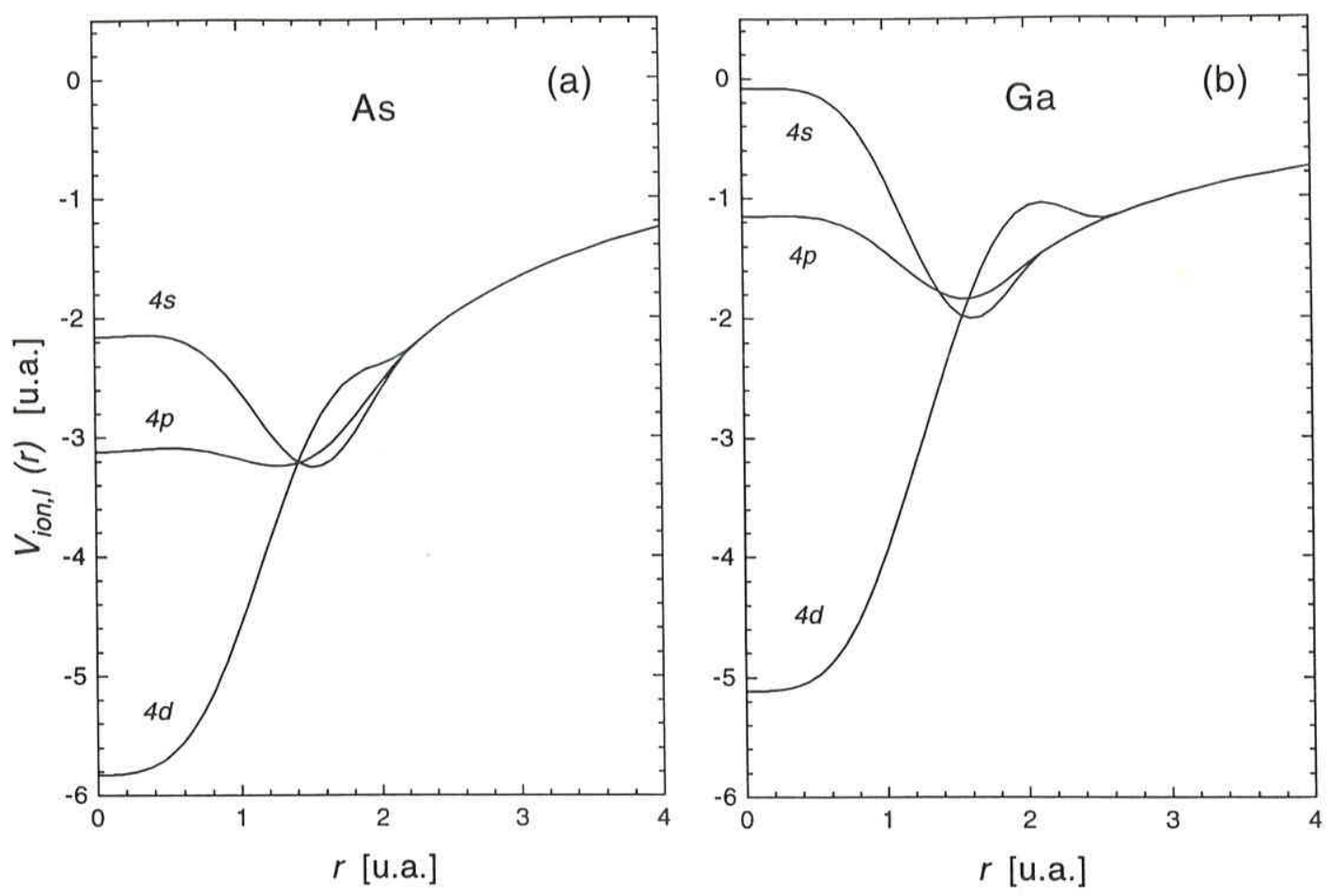

Figura A.1: Pseudopotenciais iônicos gerados pelo método de TroullierMartins. (a) Para o átomo de arsênio, com raios de corte $r_{c s}=r_{c p}=r_{c d}=2.3 a_{0}$. (b) Para o átomo de gálio, com raios de corte $r_{c s}=r_{c p}=2.3 a_{0}$ e $r_{c d}=2.8 a_{()}$, onde $a_{0}$ é o raio de Bohr $\left(a_{0}=0.529177 \AA\right)$.

para um ambiente cristalino. A perda de transferibilidade pode ser observada nos desvios da derivada logarítmica da pseudofunção de onda radial quando comparada com a derivada logarítmica da função de onda de todos os elétrons, em um determinado intervalo de energia.

Os pseudopotenciais iônicos para os diferentes átomos utilizados em nossos cálculos, foram gerados dentro da aproximação da densidade local (LDA), sem considerar polarização de spin. O pseudopotencial de gálio foi gerado a partir da configuração atômica de valência dada por, $4 s^{2} 4 p^{0.75} 4 d^{0.25}$, com raios de corte $r_{c s}=r_{c p}=2.3 a_{0}$ e $r_{c d}=2.8 a_{0}$ ( $a_{0}$ é o raio de Bohr). O aumento no raio de corte do orbital $4 d$ foi necessário para eliminar um estado "fantasma" detectado neste orbital. Para o átomo de arsênio, utilizamos configuração $4 s^{2} 4 p^{2.75} 4 d^{(0.25}$, com raios de corte $r_{c s}=r_{c p}=r_{c d}=2.3 a_{()}$. Na Figura A.1 são mostrados os pseudopotenciais iônicos destes elementos para cada orbital de valência. Aqui vemos que os pseudopotenciais não apresentam oscilações 

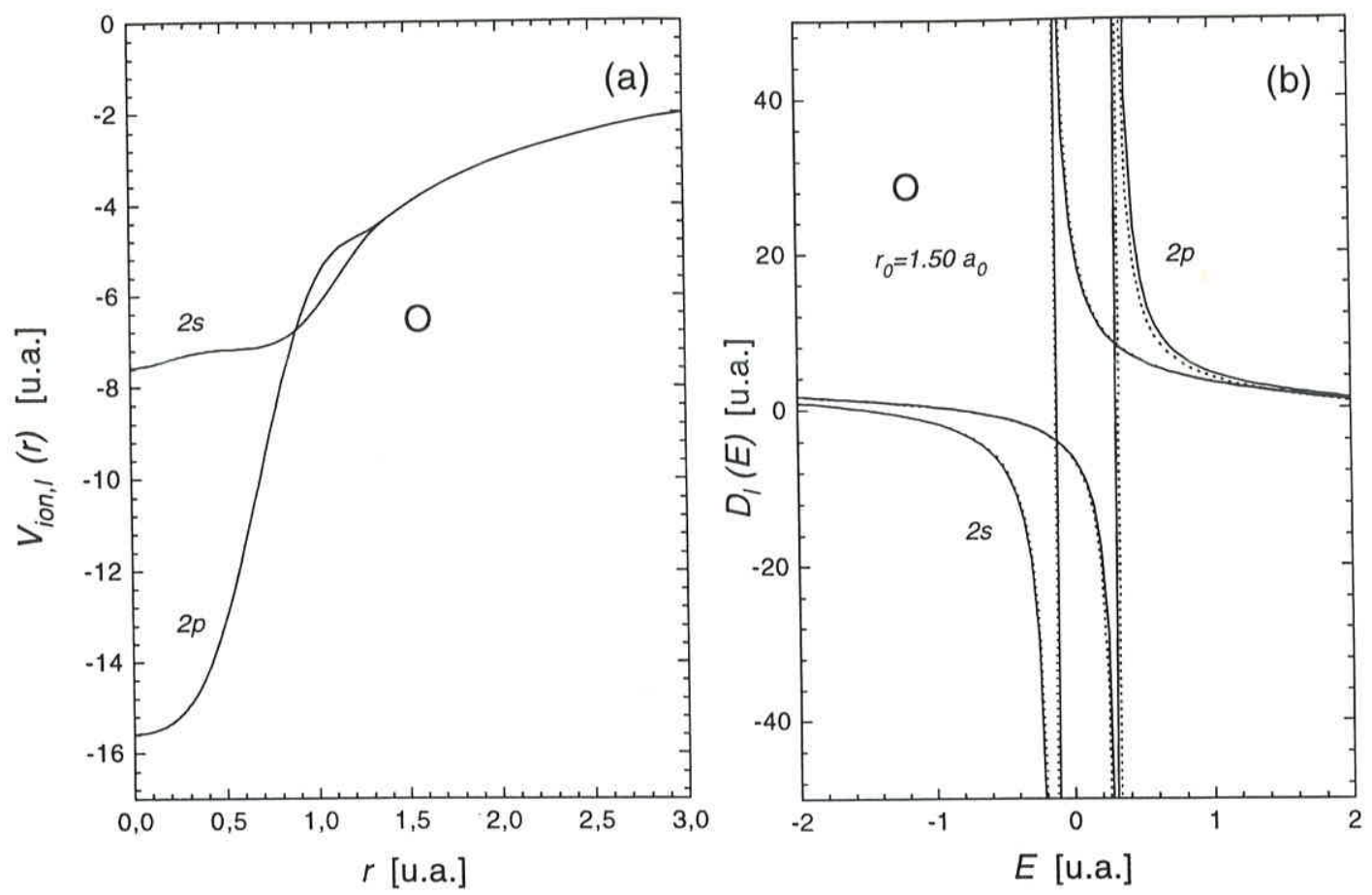

Figura A.2: (a) Pseudopotenciais iônicos dos orbitais $2 s$ e $2 p$ de oxigênio, gerados com raios de corte $r_{c s}=r_{c p}=1.45 a_{0}$. (b) Derivadas logarítmicas, obtidas $a r_{0}=1.5 a_{0}$, das pseudofunções de onda radial não locais (linha contínua) $e$ das funções de onda com todos os elétrons (linha pontilhada).

para raios menores que o raio de corte e possuem curvatura zero na origem, que são as características básicas do método de Troullier-Martins. Apesar destes raios de corte serem maiores que os utilizados em outros métodos, a transferibilidade não apresenta desvios significativos com respeito aos cálculos com todos os elétrons, o que assegura uma descrição mais realística das propriedades estruturais do cristal GaAs, como é mostrado no Apêndice B. Na Figura A.2 são mostrados os pseudopotenciais iônicos do oxigênio, gerados a partir da configuração atômica do estado fundamental, $2 s^{2} 2 p^{4}$, com raios de corte $r_{c s}=r_{c p}=1.45 a_{0)}$. Também mostramos as derivadas logarítmicas das pseudofunções de onda radial como função da energia, $D_{\ell}(E)$, definida por:

$$
D_{\ell}(E)=\left.\frac{d}{d r} \ln \left[R_{n, \ell}(r, E)\right]\right|_{r=r_{0}} .
$$

As pseudofunções $R_{n . \ell}$, foram obtidas a partir de pseudopotenciais iônicos não locais. Na figura A.2(a) podemos ver que o pseudopotencial do orbital $2 p$ é bas- 

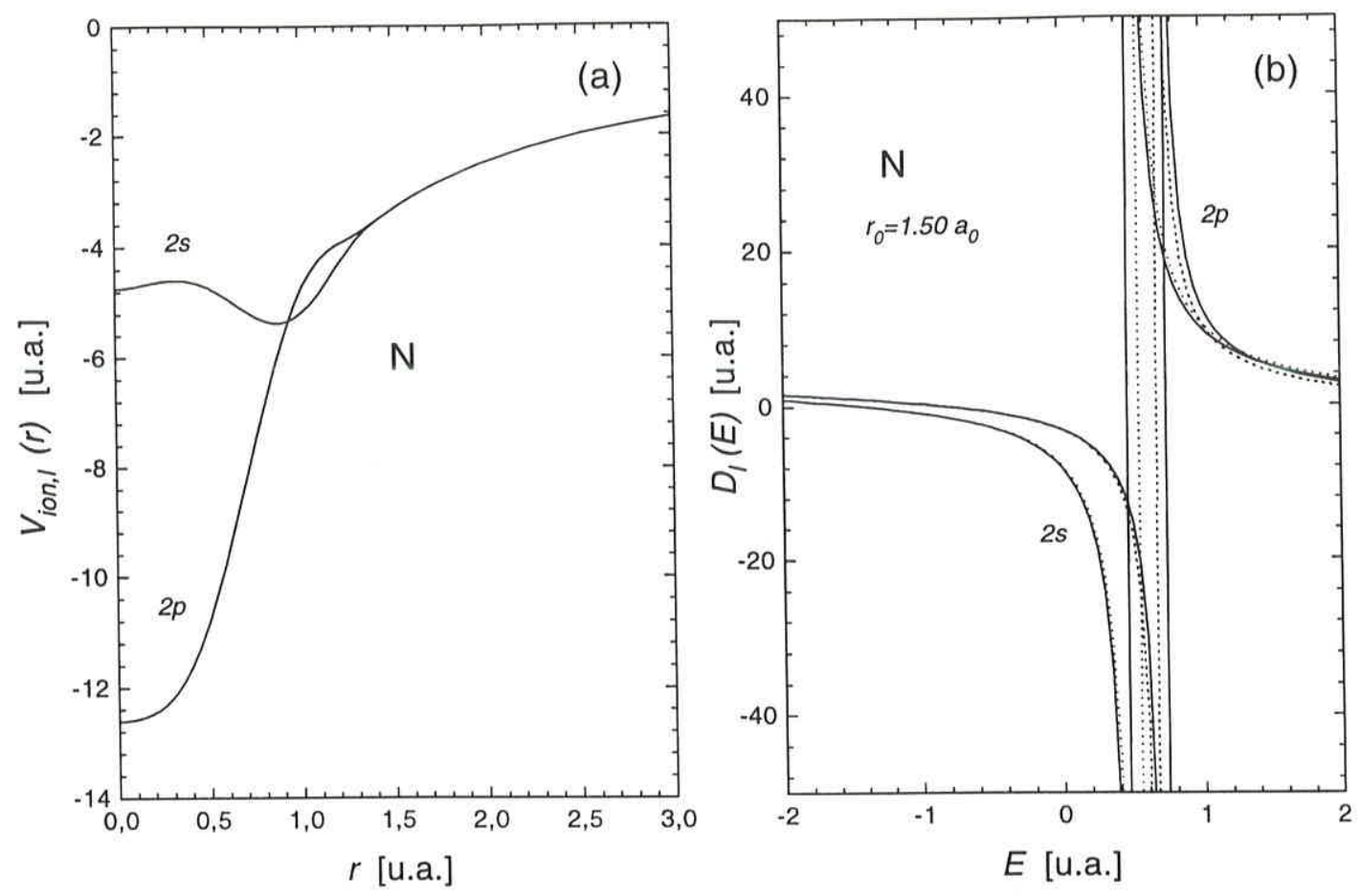

Figura A.3: (a) Pseudopotenciais iônicos dos orbitais $2 s$ e $2 p$ de nitrogênio, gerados com raios de corte $r_{c s}=r_{c p}=1.45 a_{0}$. (b) Derivadas logarítmicas, obtidas a $r_{0}=1.5 a_{0}$, das pseudofunções de onda radial não locais (linha contínua) e das funções de onda com todos os elétrons (linha pontilhada).

tante profundo. Este orbital apresenta uma energia da ordem de -15.5 hartrees, enquanto que o orbital $2 s$ é da ordem de -7.5 hartrees, bem maiores que para os átomos de arsênio e gálio, mostrando seu caráter fortemente localizado. Na Figura A.2(b) vemos que dentro do intervalo de energia considerado, não foram encontrados estados "fantasmas". Também vemos que a transferibilidade é muito boa, mostrando que a escolha dos raios de corte é adequada. Os pseudopotenciais de oxigênio gerados pelo mesmo método e parâmetros aqui apresentados, tem sido utilizado com sucesso na descrição das propriedades estruturais dos $\alpha$-quartz ${ }^{84}$

Os pseudopotenciais de nitrogênio foram gerados de forma similar ao oxigênio, na configuração do estado fundamental $2 s^{2} 2 p^{3}$, com raios de corte $r_{c s}=r_{c p}=1.45 a_{0}$. Estes são mostrados na Figura A.3(a), onde vemos que o orbital $2 p$ também é bastante profundo com uma energia da ordem de -12.5 hartrees, enquanto que o orbital $2 s$ é da ordem de -4.8 hartrees. A transferibilidade para nitrogênio também é boa, como mostra a Figura A.3(b), e da 

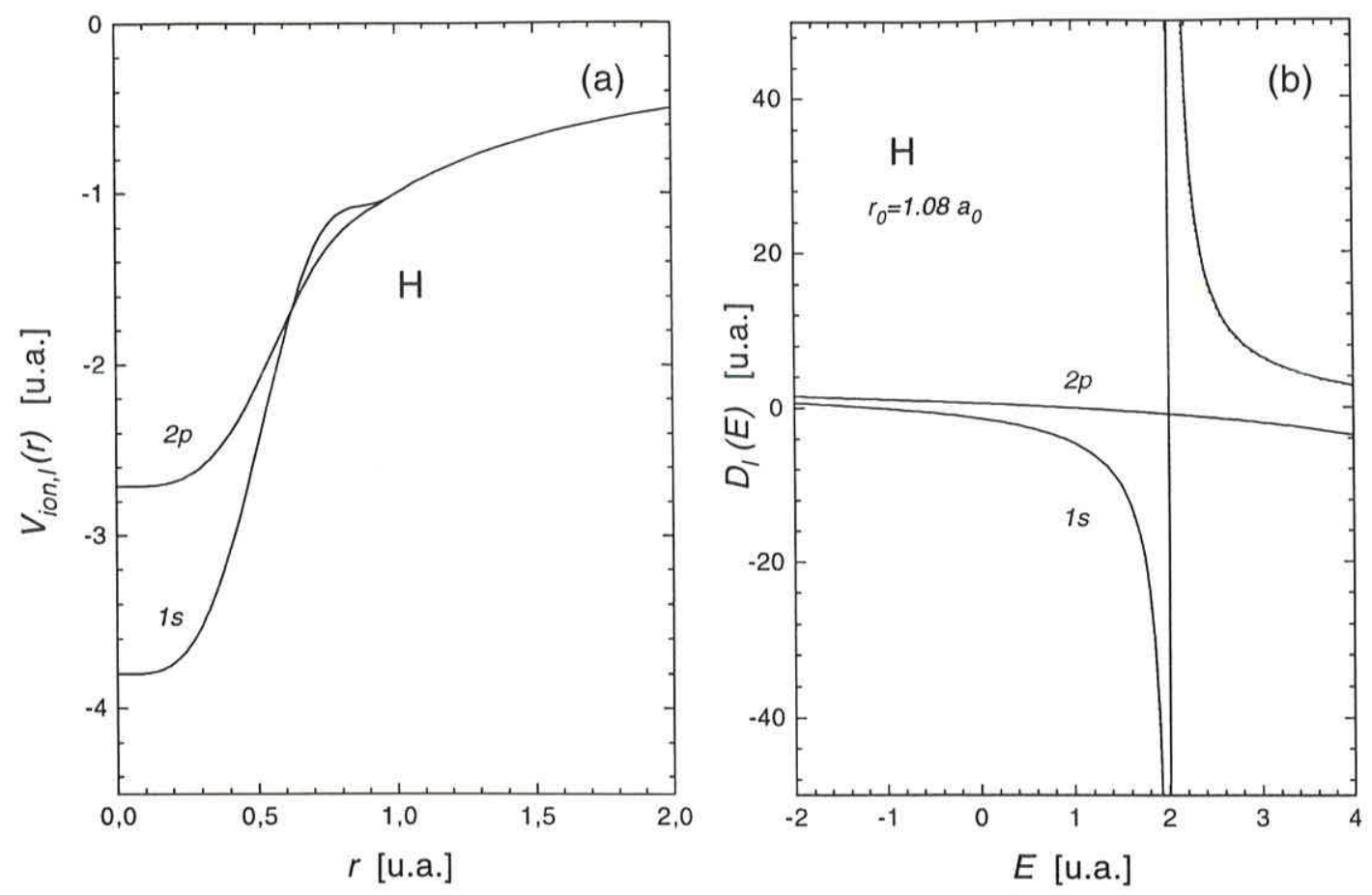

Figura A.4: (a) Pseudopotenciais iônicos dos orbitais $1 s$ e $2 p$ de hidrogênio, gerados com raios de corte $r_{c s}=r_{c p}=1.0 a_{0}$. (b) Derivadas logarítmicas, obtidas a $r_{0}=1.08 a_{0}$, das pseudofunções de onda radial não locais (linha contínua) e das funções de onda com todos os elétrons (linha pontilhada).

mesma forma que para oxigênio, não se observam estados "fantasmas".

O átomo de hidrogênio não possui estados de caroço tendo um único elétron de valência, logo descrever ele com pseudopotencial requer um certo cuidado. Usualmente em cálculos de estrutura eletrônica, para descrever o hidrogênio é usado o potencial coulombiano real, onde a divergência em $1 / r$ para $r \rightarrow 0$ é contornada fazendo o potencial convergir a um certo valor de energia. Em nossos cálculos utilizamos pseudopotencial com caroço nulo, o qual foi gerado na configuração do estado fundamental dada por $1 s^{1} 2 s^{0}$, com raios de corte $r_{c s}=r_{c p}=a_{0}$. Os pseudopotenciais iônicos e as derivadas logarítmicas são mostrados na Figura A.4. Nesta figura vemos que o pseudopotencial do único estado ocupado, $1 \mathrm{~s}$, tem um comportamento similar aos outros elementos leves, sendo mais raso, com energia da ordem de -3.8 hartrees, enquanto que o orbital $2 p$, pelo fato de estar vazio, não apresenta a localização observada para oxigênio e nitrogênio. A derivada logarítmica apresenta uma boa transferibilidade e a não aparição de estados "fantasmas". 


\section{Apêndice B}

\section{Parâmetros Estructurais do GaAs}

O cristal de arseneto de gálio ( $G a A s)$, é um semiconductor de estrutura zincblende e gap direto, i.e., o mínimo da banda de condução está situado no ponto $\Gamma$. Sua representação mais simples é constituída por uma célula unitária cúbica de fase centrada $(f c c)$ de dois átomos. Para determinarmos o parâmetro de rede teórico do cristal, calculamos a energia total do sistema de dois átomos, nas posições acima descritas, utilizando os pseudopotenciais de gálio e arsênio como gerados no Apêndice A. Como é usual nos cálculos de energia total com pseudopotencial, um dos três orbitais de valência é escolhido como local, enquanto que os outros dois são transformados na forma não local de Kleinman-Bylander. ${ }^{24}$ Nossa escolha para o orbital local foi o $p$, para os dois átomos, devido a que nesta representação foram obtidos melhores resultados para a tranferibilidade. Também incluimos uma correção para o termo de troca e correlação devido a interação entre as densidades de carga de caroço e de valência no átomo de gálio, que é conhecida como correção não linear de caroço. ${ }^{85}$ Esta interação afeta particularmente o elétron de valência do orbital $4 p$ que sofre uma blindagem artificial devido aos elétrons do orbital $3 d$ do caroço, ficando fracamente ligado. Com esta correção foram obtidas melhorias na transferibilidade do pseudopotencial do átomo de gálio e por conseqüência nos parâmetros estruturais do GaAs, como veremos mais em frente.

Para o cálculos do parâmetro de rede, utilizamos conjuntos base de ondas planas equivalentes a energias de corte de $E_{\text {cut }}=16,20,30,40 \mathrm{Ry}$, e dois pontos especiais de Chadi-Cohen ${ }^{34}$ para a soma na primeira zona de Brillouin. Na Figura B.1 são mostradas as curvas obtidas como função da energia de corte, as quais foram ajustadas a partir dos pontos teóricos com um polinômio 


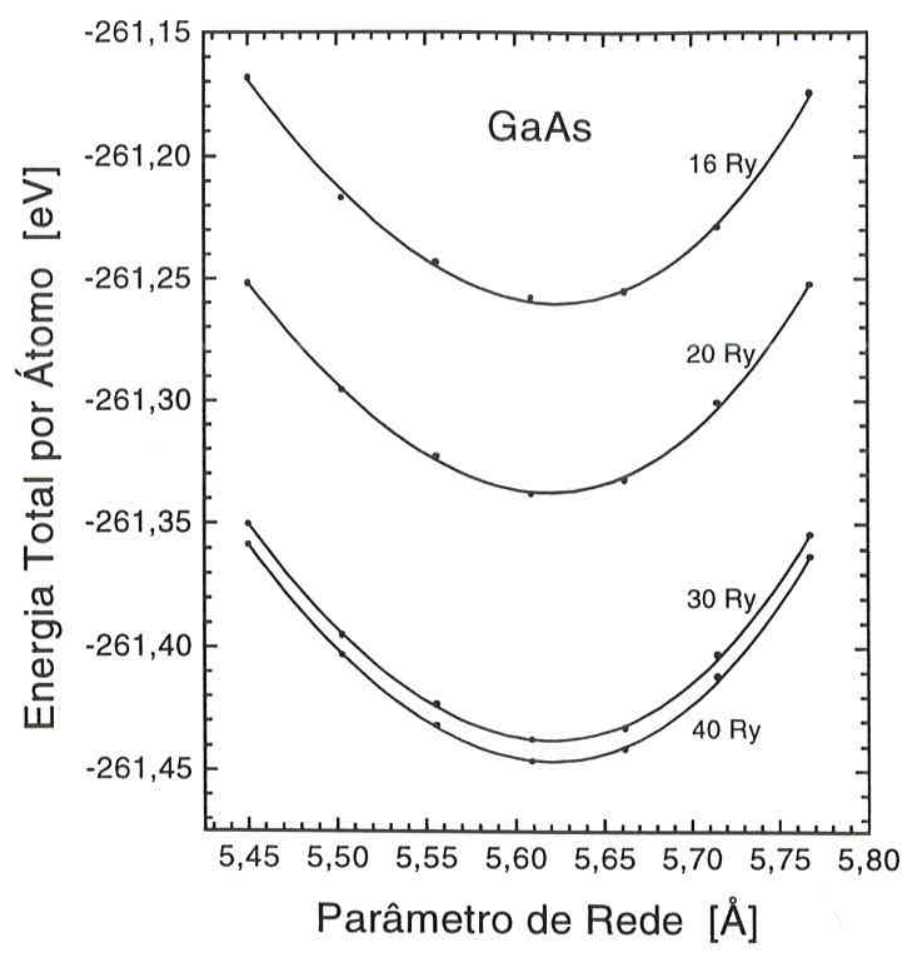

Figura B.1: Curvas de energia total por átomo como função do parâmetro de rede para GaAs, calculada para energias de corte de 16, 20, 30 e 40 Ry.

de ordem três. O ponto mínimo nas curvas nos dá o valor de equilíbrio para o parâmetro de rede teórico. A partir destas curvas também podemos obter o módulo de compressibilidade $B$, que é uma medida da deformação da estrutura cristalina submetida a pressão, definida por:

$$
B=-V \frac{d p}{d V},
$$

onde $V$ é o volume do cristal e $p$ á a pressão. A temperatura zero, a variação da energia interna para um sistema termodinâmico com um número fixo de partículas é dado por: $d U=-p d V$. Utilizando-se esta relação é possível expressar o módulo de compressibilidade em termos do volume da célula e a energia interna, que para nosso caso corresponde a energia total do sistema cristalino, assim:

$$
B=V \frac{d^{2} U}{d V^{2}} .
$$


Logo para uma célula $f c c$ de dois átomos com parâmentro de rede $a$ e volume $V\left(V=a^{3} / 4\right)$, o módulo de compressibilidade para o ponto mínimo da curva de energia total versus o parâmetro de rede é dado por:

$$
B=\frac{4}{9 a} \frac{d^{2} U}{d a^{2}} .
$$

Na Tabela B.1 apresentamos valores de equilíbrio para o parâmetro de rede, módulo de compressibilidade ou módulo de bulk e energia da banda proibida do GaAs no ponto $\Gamma$, obtidos para energias de corte de 20 e $48 \mathrm{Ry}$, as quais são comparadas com resultados experimentais. Nesta tabela também incluímos um cálculo de $G a A s$ sem considerar a correção não linear de caroço no átomo de gálio, com uma energia de corte de 20 Ry afim de avaliar a inclusão da correção. Notamos uma clara melhoria na largura da banda proibida $(\sim 10 \%)$ quando a correção é incluida, enquanto que o parâmetro de rede apresenta um aumento pouco significativo $(\sim 1 \%)$. Entretanto o módulo de bulk ultrapassa o valor experimental, tornando o cristal levemente mais duro, porém a diferença entre os módulos de bulk teóricos com e sem correção e o valor experimental, favorece a inclusão da correção. Para uma energia de corte de 48 Ry, não se observam variações significativas nos parâmetros estructurais o que mostra que a partir de um corte de $20 \mathrm{Ry}$, o cálculo de GaAs atinge a convergência. Calculamos a estrutura de bandas de energia do GaAs com uma energia de corte de $20 \mathrm{Ry}$, utilizando o parâmetro de rede obtido para esta energia ( $a=5.619 \AA$ ). Na Figura B.2 mostramos a estrutura de bandas, onde vemos os desdobramentos dos níveis de energia através da primeira zona

Tabela B.1: Parâmetro de rede (a), módulo de compressibilidade (B) e largura da banda proibida $\left(E_{\text {gap }}\right)$ do GaAs, obtidos para energias de corte $\left(E_{\text {cut }}\right)$ de 20 e 48 Ry.

\begin{tabular}{|c|c|c|c|}
\hline$E_{\text {cut }}[\mathrm{Ry}]$ & $a[\AA]$ & $B[\mathrm{Mbar}]$ & $E_{\text {gap }}[\mathrm{eV}]$ \\
\hline \hline $20^{*}$ & 5.564 & 0.720 & 1.17 \\
\hline 20 & 5.619 & 0.812 & 1.30 \\
\hline 48 & 5.609 & 0.805 & 1.30 \\
\hline Expt. $^{86.87}$ & 5.653 & 0.769 & 1.519 \\
\hline
\end{tabular}

(*) Sem correção de caroço. 


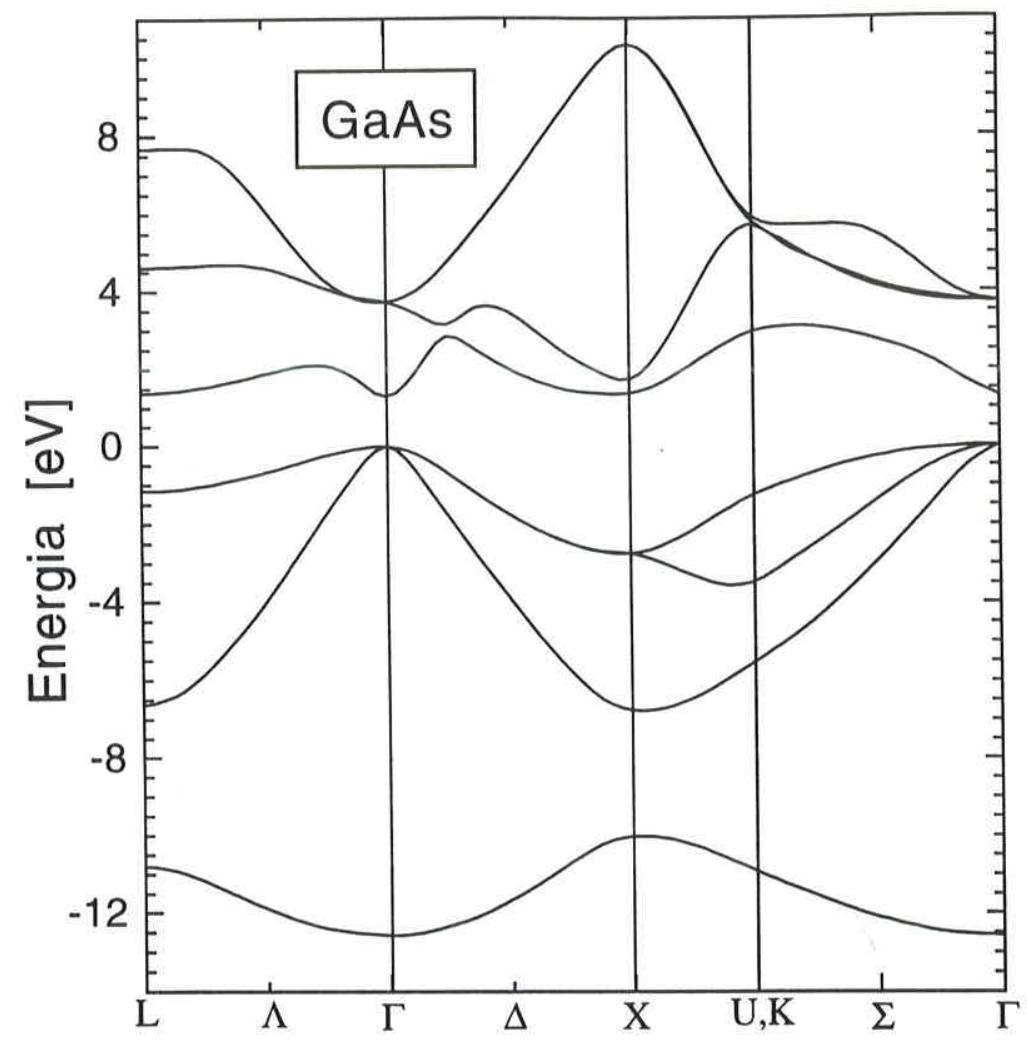

Figura B.2: Estrutura de bandas de energia do GaAs obtida com pseudopotencial não local de Troullier-Martins, para uma energia de corte de 20 Ry.

de Brillouin, em concordância com os pontos de alta simetria. A banda de valência apresenta uma largura no ponto $\Gamma$ de $12.57 \mathrm{eV}$, enquanto a banda proibida apresenta o valor de $1.30 \mathrm{eV}$. O valor experimental do gap, extrapolado a $0 \mathrm{~K}$, é de $1.519 \mathrm{eV},{ }^{87}$ e com respeito a nosso valor teórico a diferença chega a $14 \%$. Esta diferença é atribuída a aproximação da densidade local (LDA). Na Figura B.3 mostramos o gráfico de contorno da densidade de carga total para o cristal GaAs no plano [110], calculada com 48 Ry de energia de corte, em uma célula $b c c$ de 32 átomos. O espaçamento entre linhas de contorno corresponde a 12 elétrons por volume da célula $(e / \Omega)$, onde $\Omega=4764 a_{0}^{3}$. 


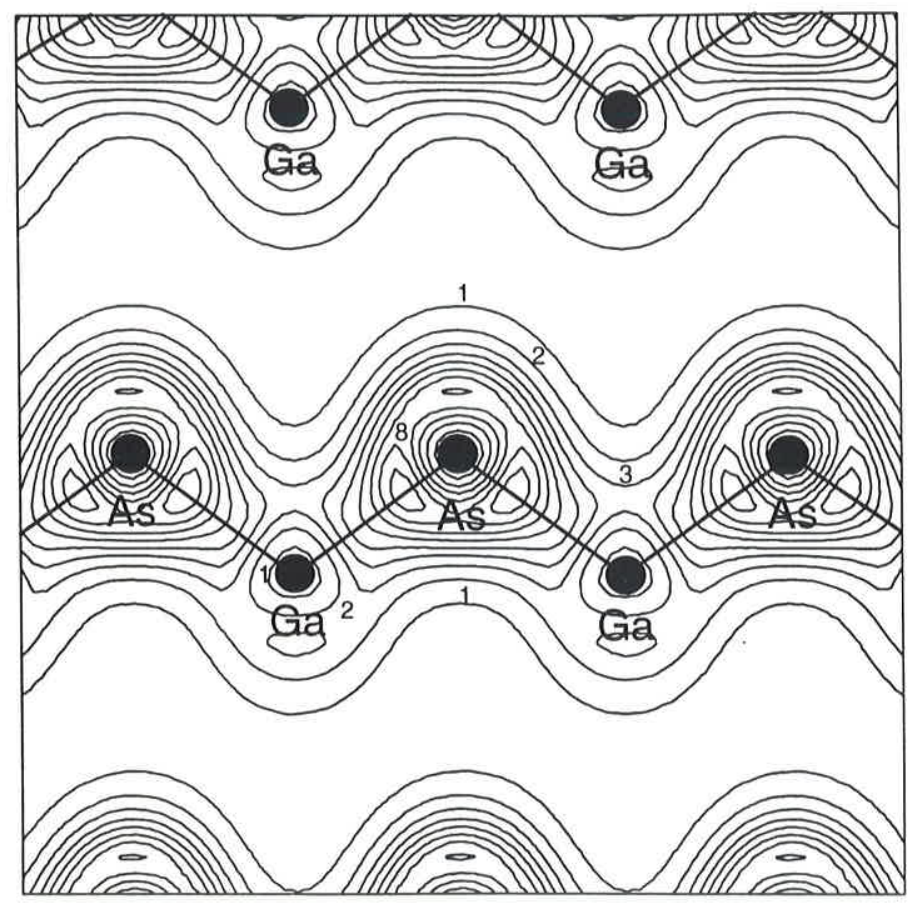

Figura B.3: Gráfico de contorno da densidade de carga total de valência no plano [110] para GaAs. Unidades em $12.0 \mathrm{e} / \Omega$. 


\section{Apêndice C}

\section{Testes de Dispersão e Convergência}

O estudo da estrutura eletrônica de defeitos em um ambiente cristalino, utilizando o espaço dos momento, requer a preservação da simetria translacional da rede. Para isto, é introduzido artificialmente uma periodicidade no sistema; construíndo-se uma supercélula de átomos hospedeiros onde o defeito está contido. Esta supercélula defeituosa é repetida no espaço, que devido as condições periódicas de contorno, é preenchido totalmente. É importante ressaltar que o tamanho da supercélula deve ser grande suficiente, de modo que a interação entre defeitos de células vizinhas seja mínima. Sistemas de impurezas como os por nós estudados, introduzem níveis de energia aproximadamente na metade do gap. A posição destes níveis não varia dentro da zona de Brillouin, mas se as distâncias entre impurezas vizinhas é tal que suas funções de onda se superpõem, os níveis de impureza apresentarão uma dispersão. Para quantificar esta dispersão fizemos cálculos de energia total para a impureza de oxigênio substitucional em $G a A s\left(G a A s: O_{A s}\right)$ utilizando três tipos de células de diferente tamanho. Uma célula cúbica simples de 8 átomos, uma célula cúbica de fase centrada $(f c c)$ de 16 átomos e uma célula cúbica de corpo centrado $(b c c)$ de 32 átomos. A distância entre impurezas de células vizinhas é de $5.56,7.87$ e $9.64 \AA$, respectivamente. Para estes três sistemas utilizamos o mesmo conjunto de ondas planas equivalente a uma energia de corte de $40 \mathrm{Ry}$. A dispersão pode ser estimada pela variação da energia do autovalor correspondente ao nível de impureza, para diferentes pontos- $k$ na primeira zona de Brillouin. Estes pontos- $k$ podem ser os proṕrios pontos especiais; assim para a célula de 8 átomos com 4 pontos especiais, obtivemos uma dispersão de $1.23 \mathrm{eV}$, para a célula de 16 átomos com 2 pontos especiais 


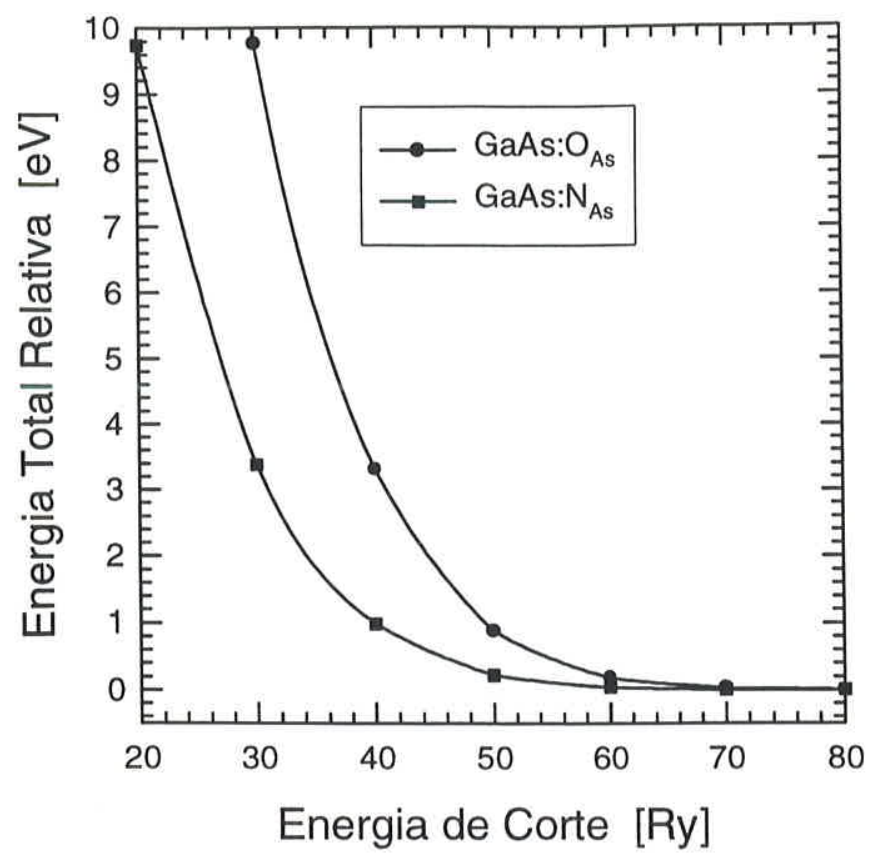

Figura C.1: Curva de convergência da energia total como função da energia de corte para os sistemas de impureza de oxigênio e nitrogênio em GaAs. A energia total zero é relativa a um cálculo com 80 Ry.

a dipersão é de $0.93 \mathrm{eV}$ e finalmente para a célula de 32 átomos com 2 pontos especiais obtivemos $0.12 \mathrm{eV}$.

Um outro teste importante que deve ser feito, é relativo a convergência dos sistemas com impurezas como função do número de ondas planas. Para isto fizemos cálculos de energia total para os sistemas de oxigênio e nitrogênio substituindo um átomo de arsênio em $G a A s$. Utilizamos uma célula cúbica de 8 átomos e 4 pontos especiais, com energias de corte variando entre 20 e 80 Ry, devido ao átomo de impureza apresentar orbitais $2 p$ fortemente localizados, como mostramos no Apêndice A. Uma célula de 8 átomos é muito pequena para tirar qualquer informação significativa das propriedades eletrônicas ou estruturais de sistemas com ela estudados, mas é apropriada para estudar a convergência, já que somente estamos interessados na variação da energia total em função do tamanho da base. Na Figura C.1 mostramos a variação da energia total relativa ao valor obtido para a maior energia de corte considerada, 80 Ry. Para o nitrogênio substituindo um arsênio (GaAs: $\left.N_{A s}\right)$, vemos que a convergência é atingida a partir de uma energia de corte de 50 Ry, que para a célula considerada corresponde a um conjunto da ordem de 7200 
ondas planas. Para o oxigênio no lugar de arsênio $\left(\mathrm{GaAs}: \mathrm{O}_{A s}\right)$, é necessário uma energia de corte maior devido a que seu pseudopotencial é mais profundo. A convergência é atingida a partir de $60 \mathrm{Ry}$, o que corresponde a aproximadamente 9400 ondas planas.

Os testes de dispersão e convergência mostram que o mais apropriado, por exemplo para descrever corretamente oxigênio em GaAs, seria utilizar uma célula de 32 átomos e uma energia de corte de 60 Ry. Com a disponibilidade de recursos computacionais que possuimos, este cálculo resulta inviável, mas acreditamos que seja possível descrever grande parte das propriedades do sistema que envolve oxigênio, que é nosso caso extremo, dentro de uma precisão aceitável, considerando um número de ondas planas equivalente a $48 \mathrm{Ry}$. Para o caso de nitrogênio consideramos uma energia de corte de 42 Ry. Uma forma usualmente utilizada para testar os pseudopotenciais e verificar se as energias de corte são adequadas, é fazer cálculos de energia total para moléculas pequenas contendo os átomos em questão, com o intuito de comparar as estruturas resultantes com dados experimentais. Fizemos cálculos de energia total para as moléculas de água $\left(\mathrm{H}_{2} \mathrm{O}\right)$, oxigênio $\left(\mathrm{O}_{2}\right)$, nitrogênio $\left(\mathrm{N}_{2}\right)$, amônia $\left(\mathrm{NH}_{3}\right)$ e hidrogênio $\left(H_{2}\right)$. As moléculas foram deixadas em uma célula cúbica vazia de $10 \AA$ de aresta, em uma geometria próxima a do equilíbrio com as coordenadas livres para relaxar. Na Tabela C.1 são mostrados nossos resultados. Aqui vemos que os valores teóricos das distâncias de ligação estão em excelente

Tabela C.1: Distâncias de ligação de moléculas ( $\left.d_{\text {teo }}\right)$, obtidas em cálculos de energia total com pseudopotencial, para energias de corte ( $E_{\text {cut }}$ ) de 42 e $48 R y$.

\begin{tabular}{|c|c|c|c|}
\hline Molécula & $E_{\text {cut }}[\mathrm{Ry}]$ & $d_{\text {teo. }}[\AA]$ & $d_{\text {expt. }}{ }^{88}[\AA]$ \\
\hline \hline $\mathrm{H}_{2}$ & 42 & 0.770 & 0.7414 \\
\hline $\mathrm{N}_{2}$ & 42 & 1.106 & 1.0977 \\
\hline $\mathrm{NH}_{3}$ & 42 & 1.027 & 1.0124 \\
\hline $\mathrm{O}_{2}$ & 48 & 1.238 & 1.2074 \\
\hline $\mathrm{H}_{2} \mathrm{O}$ & 48 & 0.976 & 0.9575 \\
\hline
\end{tabular}

concordância com os valores experimentais, com diferenças menores que 4\%, o que mostra que as energias de corte utilizadas são adequadas. Para a molécula de água obtivemos um ângulo entre ligações de $103.8^{\circ}$, bastante próximo de seu valor experimental, que é de $104.51^{\circ}$, enquanto que para a molécula de amônia os três ângulos $H-N$ - $H$ foram obtidos em $106.6^{\circ}$, onde o valor experimental correspondente é de $106.67^{\circ} .^{88}$ 


\section{Apêndice D}

\section{Energia de Formação}

O cálculo da energia de formação de um sistema cristalino contendo defeitos, permite estimar quais são as condições mais favoráveis para a incorporação destes defeitos. No caso de impurezas substitucionais, tais como oxigênio e nitrogênio em $G a A s$, a energia de formação pode ser determinada pela abundância relativa dos átomos constituentes no meio em que o cristal é crescido. Em um contexto termodinâmico, estas abundâncias podem ser descritas em termos dos potenciais químicos dos reservatórios atômicos $\left(\mu_{G a}\right.$, $\mu_{A s}, \mu_{O}$ e $\mu_{N}$ ), obtidos a partir da energia livre de Gibbs do sistema, definida por:

$$
G \equiv E-T S+p V
$$

onde $E, S$ e $V$ são as variáveis extensivas de energia total, entropia e volume, respectivamente, enquanto que $T$ e $p$ correspondem as variáveis intensivas de temperatura e pressão. Considerando um sistema homogêneo em equilíbrio termodinâmico, constituído por $n$ diferentes tipos de átomos em um volume $V$ com energia $E$, as variáveis intensivas do sistemas serão:

$$
G=G\left(T, p, N_{1}, N_{2}, \ldots, N_{n}\right),
$$

onde $N_{i}$ é o número de átomos da espécie $i$. Definimos a energia de formação como:

$$
E_{\text {form }} \equiv G\left(T, p, N_{1}, N_{2}, \ldots, N_{n}\right)-\sum_{i=1}^{n} G\left(T, p, N_{i}\right)
$$


onde $G\left(T, p, N_{i}\right)$ é a energia livre de um sistema com uma única espécie atômica $i$, que definimos como reservatório. Como neste caso $N_{i}$ é variável extensiva, podemos escrever:

$$
G\left(T, p, N_{i}\right)=N_{i} g_{i}(T, p),
$$

onde $g_{i}(T, p)$ é a energia livre por átomo do reservatório da espécie atômica $i$. O potencial químico deste sistema é dado por:

$$
\mu_{i}=\left(\frac{\partial G}{\partial N_{i}}\right)_{T, p}=g_{i}(T, p),
$$

i.e., o potencial químico do reservatório da espécie atômica $i$, é igual à sua energia livre por átomo. Logo a equação (D.3) pode ser reescrita como:

$$
E_{\text {form }}=G\left(T, p, N_{1}, N_{2}, \ldots, N_{n}\right)-\sum_{i=1}^{n} N_{i} \mu_{i} .
$$

$\mathrm{Na}$ fase condensada o termo $p V$ em (D.1) é considerado nulo devido às forças serem pequenas para o cristal em equilíbrio. Por outro lado o termo TS em princípio deveria ser considerado, mas também é ignorado devido a ser geralmente pequeno ${ }^{89}$ e tende a se cancelar quando energias livres relativas são subtraidas no cálculo da energia de formação. ${ }^{90,91}$ Desta forma a energia livre de um sistema de $n$ espécies atômicas, é aproximado pela energia total do sistema a $T=0$ :

$$
G\left(T, p, N_{1}, N_{2}, \ldots, N_{n}\right) \approx E_{\text {total }}\left(T=0, N_{1}, N_{2}, \ldots, N_{n}\right) .
$$

Finalmente a energia de formação pode ser escrita como a diferença entre a energia total a $T=0$ de um sistema formado por $n$ espécies atômicas e suas respectivas energias em um estado de referência. A referência corresponde aos reservatórios cujas energias (a $T=0$ ) são dadas pelos potencias químicos:

$$
E_{\text {form }}=E_{\text {total }}\left(T=0, N_{1}, N_{2}, \ldots, N_{n}\right)-\sum_{i=1}^{n} N_{i} \mu_{i} .
$$

Para a impureza isolada de oxigênio substitucional em GaAs no estado de carga $q$, estudada a partir de cálculos de energia total de primeiros princípios, a energia de formação pode ser escrita como: 


$$
E_{\text {form }}\left(O_{A s}^{q}\right)=E_{\text {total }}\left(O_{A s}^{q}\right)-n_{G a} \mu_{G a}-n_{A s} \mu_{A s}-\mu_{O}-q \mu_{e},
$$

onde $E_{\text {total }}\left(O_{A s}^{q}\right)$ é a energia total calculada com a supercélula contendo a impureza $O_{A s}^{q} \cdot n_{G a}\left(n_{A s}\right)$ representam o número de átomos de $G a(A s)$ na célula e $\mu_{e}$ é o potencial químico eletrônico ou a posição do nível de Fermi que funciona como variável. A equação (D.9) ilustra a forma básica da expressão para a energia de formação de impurezas substitucionais isoladas, a qual pode ser generalizada facilmente para outro tipo de defeitos.

Como os potenciais químicos são variáveis, é necessário determinar os intervalos de variação a que estão sujeitos considerando as fases que seus respectivos átomos podem formar. Para o cristal hospedeiro devemos considerar os seguintes vínculos: $\mu_{G a}+\mu_{A s}=\mu_{G a A s}, \mu_{G a} \leq \mu_{G a(b u l k)}$ e $\mu_{A s} \leq \mu_{A s(b u l k)}$, que representam respectivamente o equilíbrio termodinâmico dos átomos isolados da gálio e arsênio com respeito ao cristal $G a A s$, e a condição de não precipitação destes átomos nas suas respectivas fases bulk. O intervalo de variação dos potenciais químicos de gálio e arsênio e, portanto a estequiometria do sistema, podem ser descritos facilmente introduzindo um parâmetro $\lambda$ que varia entre zero e um, tal que:

$$
\begin{aligned}
& \mu_{G a}=\mu_{G a(b u l k)}-\lambda \Delta H_{f} \\
& \mu_{A s}=\mu_{A s(b u l k)}-(1-\lambda) \Delta H_{f},
\end{aligned}
$$

onde $\Delta H_{f}$ é o calor de formação do GaAs, calculado em $0.61 \mathrm{eV} / 2$ átomos, enquanto que seu valor experimental é de $0.74 \mathrm{eV} / 2$ átomos. ${ }^{92} \mathrm{~A}$ introdução do parâmetro $\lambda$ permite simular as condições de formação do defeito em um ambiente rico em gálio $(\lambda=0)$ ou rico em arsênio $(\lambda=1)$. Para determinar um valor máximo ou limite superior para o potencial químico da impureza, é necessário explorar todos os possíveis compostos que esta pode formar com os átomos hospedeiros no qual se liga, de forma a assemelhar o melhor possível sua interação no sistema de defeito. Para o caso específico do defeito $O_{A s}$ em $G a A s$, um valor máximo para $\mu_{O}$ pode ser obtido a partir do cálculo da energia total do composto óxido de gálio, $\mathrm{Ga}_{2} \mathrm{O}_{3}$, já que este óxido reproduz as ligações do oxigênio quanto substitui um arsênio em $\mathrm{GaAs}$.

Para a obtenção de um limite superior para os potenciais químicos de gálio e arsênio, fizemos cálculos de energia total destes dois elementos nas 

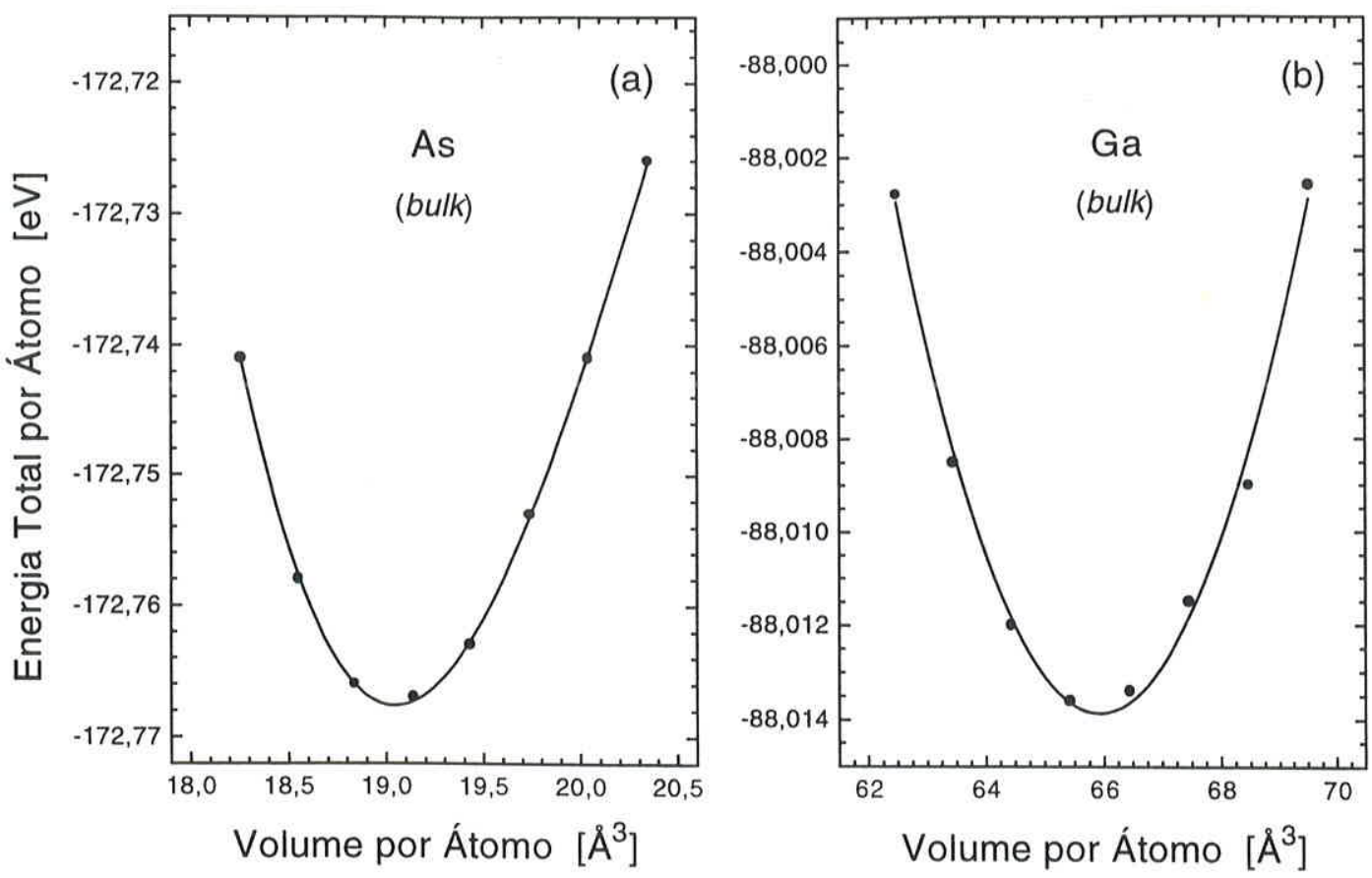

Figura D.1: Curvas de energia total em função do volume do cristal. (a) Arsênio bulk, calculado com 48 pontos especiais. (b) Gálio bulk, calculado com 74 pontos especiais. A energia de corte nos dois casos é de 48 Ry.

suas respectivas fases bulk, utilizando os mesmos pseudopotenciais e energia de corte que nos cálculos dos sistemas de impureza. Arsênio bulk forma uma estrutura romboédrica com parâmetros experimentais dados por ${ }^{93} a=4.131 \AA$ e $\alpha=54.16^{\circ}$, e dois átomos por célula unitária. Devido ao seu caráter metálico, utilizamos 48 pontos especiais de Monkhorst-Pack ${ }^{45}$ para determinar corretamente sua estrutura eletrônica. Nossos resultados teóricos para os parâmetros, obtidos após a obtenção do mínimo de energia e permitindo uma posterior relaxação, foram: $a=3.622 \AA$ e $\alpha=44.94^{\circ}$. A energia total obtida foi -172.790 eV/átomo. Gálio bulk forma uma estrutura ortorrómbica de oito átomos por célula unitária, com parâmetros experimentais ${ }^{93} a=4.511 \AA$, $u=0.0785$ e $v=0.1525$. No cálculo da estrutura eletrônica foram utilizados 74 pontos especiais de Monkhorst-Pack, ${ }^{45}$ nossos resultados para os parâmetros foram $a=4.432 \AA, u=0.0821$ e $v=0.1556$, com energia total de -88.053 eV/átomo. Na Figura D.1 são mostradas as curvas de energia total calculadas para as duas estruturas. Um limite superior para $\mu_{O}$ foi obtido a partir do cálculo do potencial químico de óxido de gálio $\mu_{G_{2} \mathrm{O}_{3}}$, considerando-se a seguinte relação: 

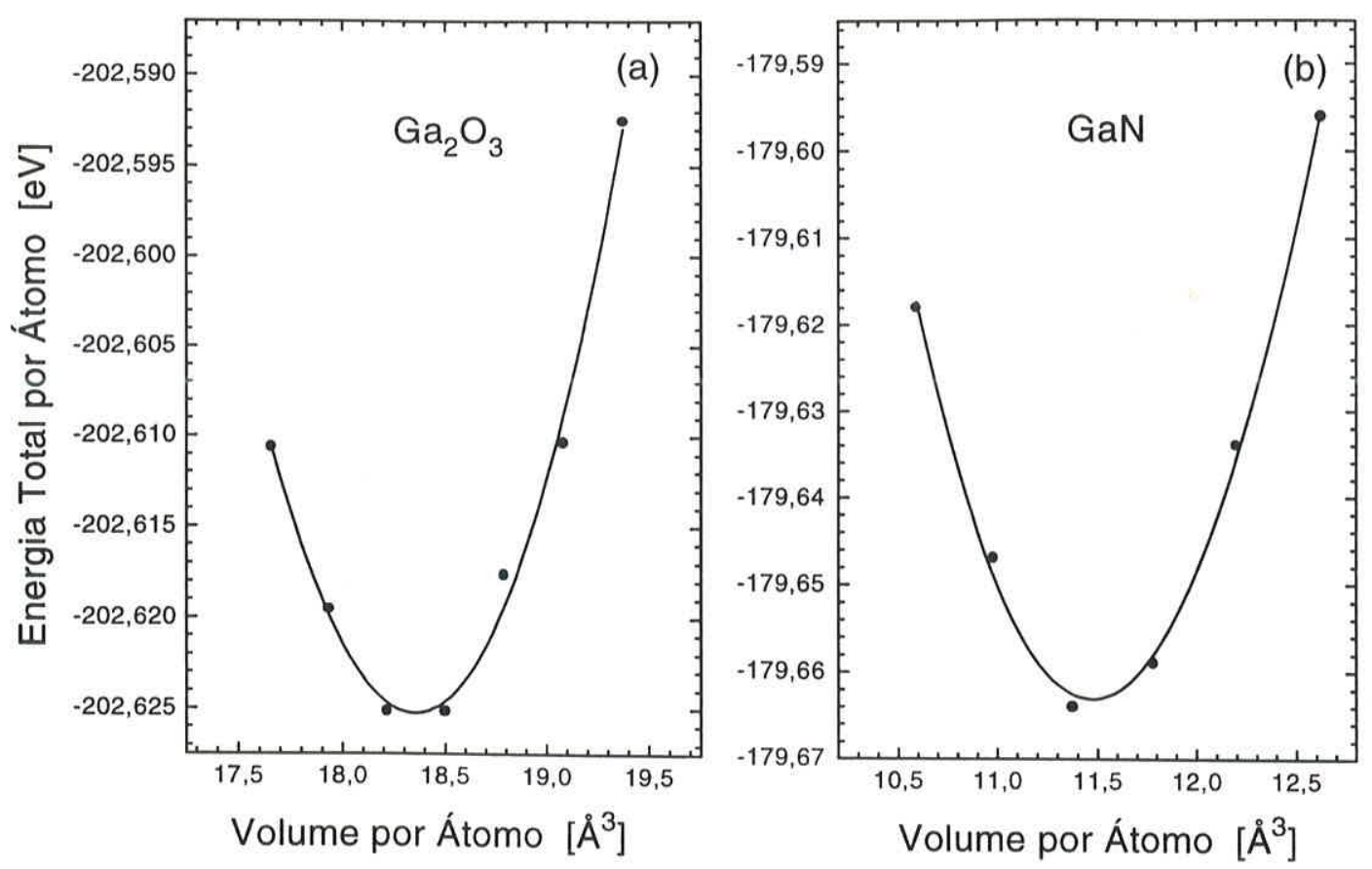

Figura D.2: Curvas de energia total em função do volume do cristal. (a) Oxido de gálio $\left(\mathrm{Ga}_{2} \mathrm{O}_{3}\right)$, calculado com 74 pontos especiais e 48 Ry de energia de corte. (b) Nitreto de gálio (GaN), calculado com 10 pontos especiais e 42 Ry de energia de corte.

$$
2 \mu_{\text {Ga(bulk })}+3 \mu_{O}^{\max }=\mu_{G a_{2} O_{3}} .
$$

Óxido de gálio forma uma estrutura romboédrica, que pode ser representada equivalentemente por uma estrutura hexagonal com parâmetros experimentais $a=4.979 \AA$ e $c=13.429 \AA{ }^{94}$ em uma célula unitária de 6 átomos; os cálculos de energia total foram realizados considerando 74 pontos especiais de Monkhorst-Pack com uma energia de corte de $48 \mathrm{Ry}$. Os resultados téoricos dos parâmetros estruturais obtidos no ponto mínimo da curva de energia total são $a=4.817 \AA$ e $c=12.992 \AA$. A energia total do sistema após a relaxação é de $-203.28 \mathrm{eV} /$ átomo. Na Figura D.2(a) é mostrada a curva de energia total para esta estrutura. Para obter um limite superior para o potencial químico da impureza substitucional de nitrogênio em $G a A s$, devemos considerar independentemente os dois possíveis sítios substitucionais que ela pode ocupar afim de assemelhar corretamente sua interação com o cristal. 
Para o defeito $N_{A s}$ em $G a A s$, um limite superior para $\mu_{N}$ foi obtido a partir do cálculo do potencial químico de nitreto de gálio, $G a N$. Este é um cristal semicondutor que pode formar estrutura zinc-blende ou wurtzite, onde para a fase zinc-blende o parâmero de rede experimental ${ }^{95}$ é de $a=4.52 \AA$ e o módulo de bulk de $B=1.90 \mathrm{Mbar}$. Os cálculos de energia total para este cristal foram feitos utilizando-se uma célula cúbica $f c c$ de dois átomos, 10 pontos especiais de Chadi-Cohen ${ }^{34}$ na primeira zona de Brillouin e uma energia de corte de 42 Ry. Nossos resultados para o parâmetro de rede e módulo de bulk são: $a=4.52 \AA$ e $B=1.00$ Mbar. A energia total para $G a N$ foi obtida a partir do mínimo da curva de energia total em função do volume da célula, Figura D.2(b), e o valor obtido é - $179.663 \mathrm{eV} /$ átomo. Logo um limite superior para potencial químico do nitrogênio no sistema considerado é obtido a partir da relação:

$$
\mu_{N}^{\max }+\mu_{G a(b u l k)}=\mu_{G a N} .
$$

Por último, um valor máximo para o potencial químico de nitrogênio quando forma o defeito $N_{G a}$ em $G a A s$, foi obtido a partir do cálculo da energia total da molécula de nitrogênio $N_{2}$. Esta foi calculada em uma caixa de $10 \AA$ de aresta, utilizando 4 pontos especiais de Chadi-Cohen e uma energia de corte de 42 Ry. A energia total obtida na geometria de equilíbrio é de $-538.936 \mathrm{eV}$. Finalmente um limite superior para $\mu_{N}$ neste sistema é obtido utilizando a relação:

$$
2 \mu_{N}^{\max }=\mu_{N_{2}} .
$$




\section{Bibliografia}

[1] J. Bernholc, N. O. Lipari e S. T. Pantelides, Phys. Rev. Lett. 41, 895 (1978).

[2] J. Bernholc, N. O. Lipari e S. T. Pantelides, Phys. Rev. B 21, 3545 (1980).

[3] G. A. Baraff e M. Schlüter, Phys. Rev. Lett. 41, 895 (1978).

[4] G. A. Baraff e M. Schlüter, Phys. Rev. B 19, 4965 (1979).

[5] G. D. Watkins e J. W. Corbett, Phys. Rev. 121, 1001 (1961); J. W. Corbett, G. D. Watkins, R. M. Chrenko e R. S. McDonald, Phys. Rev. 121, 1015 (1961).

[6] G. A. Baraff, E. O. Kane e M. Schluter, Phys. Rev. Lett. 47, 601 (1981).

[7] J. Schneider, B. Dischler, H. Seelewind, P. M. Mooney, J Lagowski, M. Matsui, D. R. Beard e R. C. Newman, Appl. Phys. Lett. 54, 1442 (1989).

[8] R. Car e M. Parrinello, Phys. Rev. Lett. 55, 2471 (1985).

[9] P. Hohemberg e W. Kohn, Phys. Rev. 136, B864 (1964).

[10] Advances Quantum Chemestry, Vol. 21, editado por S. B. Trickey (Academic Press inc., San Diego, 1990), pp. 7-26.

[11] W. Y. Han, Y. Lu, H. S. Lee, W. M. Cole, S. N Schauer, R. P. Moerkirk, K. A. Jones e L. W. Yang, Appl. Phys. Lett. 61, 87 (1992).

[12] J. Wagner, M. Maier, Th. Lauterbach, K. H. Bachem, A. Fischer, K. Ploog, G. Mörsch e M. Kamp, Phys. Rev. B 45, 9120 (1992).

[13] D. M. Kozuch, M. Stavola, S. J. Pearton, C. R. Abernathy e W. S. Hobson, J. Appl. Phys. 73, 3716 (1993). 
[14] B. Pajot e Ch. Song, Phys. Rev. B 45, 6484 (1992).

[15] B. Clerjaud, D. Côte, W. -S. Hahn, A. Lebkiri, W. Ulrici e D. Wasik, Phys. Rev. Lett. 77, 4930 (1996).

[16] B. Clerjaud, D. Côte, W. -S. Hahn, A. Lebkiri, W. Ulrici e D. Wasik, Phys. Stat. Sol. (a), 159, 121 (1997).

[17] R. Murray, Physica B 170, 115 (1991).

[18] W. Kohn e L. J. Sham, Phys. Rev. 140, A1133 (1965).

[19] J. C. Phillips e L. Kleinman, Phys. Rev. 116, 287 (1959).

[20] J. Perdew e A. Zunger, Phys. Rev. B 23, 5048 (1981).

[21] D. M. Ceperley e B. J. Alder, Phys. Rev. Lett. 45, 566 (1980).

[22] C. Herring, Phys. Rev. 57, 1169 (1940).

[23] D. R. Hamann, M. Schlüter e C. Chiang, Phys. Rev. Lett. 43, 1494 (1979).

[24] L. Kleinman e D. M. Bylander, Phys. Rev. Lett. 48, 1425 (1982).

[25] X. Gonze, P. Käckell e M. Scheffler, Phys. Rev. B 41, 12264 (1990).

[26] D. M. Bylander e L. Kleinman, Phys. Rev. B 41, 907 (1990).

[27] N. Troullier e J. L. Martins, Sol. State Comm. 74, 613 (1990).

[28] G. P. Kerker, J. Phys. C 13, L189 (1980).

[29] J. R. Chelikowsky, H. E. King, Jr., N. Troullier, J. M. Martins e J. Glinnemann, Phys. Rev. Lett. 65, 3309 (1990).

[30] K. M. Glassford, N. Troullier, J. M. Martins e J. Chelikowsky, Sol. State Comm. 76, 635 (1990).

[31] J. Ihm, A. Zunger e M. Cohen, J. Phys. C 12, 4409 (1979).

[32] P. P. Ewald, Ann. Phys. (Leipzig) 64, 253 (1921).

[33] A. Baldereschi, Phys. Rev. B 7, 5212 (1973).

[34] D. J. Chadi e M. L. Cohen, Phys. Rev. B 8, 5747 (1973). 
[35] H. Hellmann, Einführung in der Quanten Theorie (Deuticke, Leipzig, 1937).

[36] R. P. Feynman, Phys. Rev. 56, 340 (1939).

[37] J. C. Slater, J. Chem. Phys. 57, 2389 (1972).

[38] M. C. Peyne, M. P. Teter, D. C. Allan, T. A. Arias e J. D. Joannopoulos, Rev. Mod. Phys. 64, 1045 (1992).

[39] G. Song, W. Ge, D. Jiang e G. Hsu, Appl. Phys. Lett. 50, 1666 (1987).

[40] H. Ch. Alt, Phys. Rev. Lett. 65, 3421 (1990).

[41] P. W. Anderson, Phys. Rev. Lett. 34, 953 (1975).

[42] M. Linde, J. M. Spaeth e H. Ch. Alt, Appl. Phys. Lett. 67, 662 (1995).

[43] M. Linde, H. Ch. Alt e J. M. Spaeth, Mater. Sci. Forum 196-201, 213 (1995).

[44] R. Jones e S. Öberg, Phys. Rev. Lett. 69, 136 (1992).

[45] H. J. Monkhorst e J. D. Pack, Phys. Rev. B 13, 5188 (1976).

[46] R. Stumpf e M. Scheffler, Comput. Phys. Comm. 79, 447 (1994).

[47] H. A. Jahn e E. Teller, Proc. Roy. Soc. London 161, 220 (1937).

[48] M. Lannoo, Phys. Rev. B 25, 2987 (1982).

[49] S. Canuto e A. Fazzio, Phys. Rev. B 33, 4432 (1986).

[50] S. B. Zhang e J. E. Northrup, Phys. Rev. Lett. 67, 2339 (1991).

[51] T. Mattila e R. M. Nieminen, Phys. Rev. B 54, 16676 (1996).

[52] D. Vanderbilt, Phys. Rev. B 41, 7892 (1990); K. Laasonen et. al., ibid. 47, 10142 (1993).

[53] G. B. Bachelet, D. R. Hamann e Schlüter, Phys. Rev. B 26, 4199 (1982);

D. R. Hamann, Bull. Am. Phys. Soc 33, 803 (1988).

[54] D. G. Thomas e J. J. Hopfield, Phys. Rev. 150, 680 (1966). 
[55] R. Schwabe, W. Seifert, F. Bugge, R. Bindemann, V. F. Agekyan e S. V. Pogarev, Sol. State Comm. 55, 167 (1985).

[56] T. Makimoto, H. Saito e N. Kobayashi, Jpn. J. Appl. Phys. 36, 1694 (1997).

[57] T. Shima, S. Kimura, T. Iida, A. Obara, Y. Makita, K. Kudo, K. Tanaka, Nucl. Instr. and Meth. in Phys. Res. B 120, 293 (1996).

[58] J. Miao, I. M. Tiginyanu, H. L. Hartnagel, G. Irmer, J. Monecke e B. L. Weiss, Appl. Phys. Lett. 70, 847 (1997).

[59] R. Faulkner, Phys. Rev. 175, 991 (1968).

[60] M. Jaros e S. Brand, J. Phys. C 12, 525 (1979).

[61] M. J. Caldas, J. Dabrowski, A. Fazzio e M. Scheffler, Phys. Rev. Lett. 65, 2046 (1990).

[62] C. -T. Sah, Y. -C. Sun e J. -T. Tzou, Appl. Phys. Lett. 43, 204 (1983); J. Appl. Phys. 54, 5864 (1983).

[63] J. I. Pankove, D. E. Carlson, J. E. Berkeyheiser e R. O. Wance, Phys. Rev. Lett. 51, 2224 (1983); J. I. Pankove, R. O. Wance e J. E. Berkeyheiser, Appl. Phys. Lett. 45, 1100 (1984).

[64] Hydrogen in Semiconductors, Semiconductors and Semimentals, Vol. 34, Editado por J. I. Pankove e N. M. Johnson (Academic Press, San Diego, 1991).

[65] A. Amore Bonapasta, Phys. Rev. B 51, 4172 (1995).

[66] A. Amore Bonapasta, Phys. Rev. B 48, 8771 (1993).

[67] S. J. Breuer, R. Jones, P. R. Briddon e S. Öberg, Phys. Rev. B 53, 16289 (1996).

[68] Sun-Ghill Lee e K. J. Chang, Phys. Rev. B 54, 8522 (1996).

[69] R. M. Silverstein, G. C. Bassler e T. C. Morril, Spectrometric Identification of Organic Compounds, (John Wiley \& Sons, New York, 1991).

[70] C. G. Van de Walle, Phys. Rev. B 49, 4579 (1994).

[71] K. J. Chang e D. J. Chadi, Phys. Rev. Lett. 62, 937 (1989). 
[72] C. G. Van de Walle, Y. Bar-Yam e S. T. Pantelides, Phys. Rev. Lett. 60, 2761 (1988); C. G. Van de Walle, P. J. H. Danteneer, Y. Bar-Yam e S. T. Pantelides, Phys. Rev. B 39, 10791 (1989).

[73] K. J. Chang e D. J. Chadi, Phys. Rev. B 40, 1164 (1989).

[74] P. Briddon, R. Jones e G. M. S. Lister, J. Phys. C 21, L1027 (1988).

[75] J. D. Holbech, B. B. Nielsen, R. Jones, P. Sitch e S. Öberg, Phys. Rev. Lett. 71, 875 (1993).

[76] M. Budde, B. B. Nielsen, R. Jones, J. Goss e S. Öberg, Phys. Rev. B 54, 5485 (1996).

[77] C. H. Seager, R. A. Anderson e D. K. Hahn, J. Appl. Phys. 68, 3268 (1990).

[78] Z. N. Liang, P. J. H. Denteneer e L. Niesen, Phys. Rev. B 52, 8864 (1995).

[79] L. Korpás, J. W. Corbett e S. K. Estreicher, Superlatt. Microstruct., 10, 121 (1991); L. Korpás, J. W. Corbett e S. K. Estreicher, Phys. Rev. B 46, 12365 (1991).

[80] A. Amore Bonapasta, Phys. Rev. B 46, 10119 (1992).

[81] L. Pavesi e P. Giannozzi, Phys. Rev. B 46, 4621 (1992).

[82] J. Vetterhöffer, J. Wagner e J. Weber, Phys. Rev. Lett. 77, 5409 (1996).

[83] A. Amore Bonapasta, Phys. Rev. B 54, 7618 (1996). 83-875511992.

[84] J. R. Chelikowski, H. E. King, Jr., N. Troullier, J. L. Martins e J. Glinnemann, Phys. Rev. Lett. 65, 3309 (1990).

[85] S. Louie, S. Froyen e M. J. Cohen, Phys. Rev. B 26, 1738 (1982).

[86] R. I. Cottan e G. A. Saunders, J. Phys. C 6, 2105 (1973).

[87] Landolt-Börstein, New Series Group III/22a, Vol. 17, (Springer-Verlag, Berlin, 1982).

[88] CRC Handbook of Chemistry and Physics, $73^{a}$ ed., editado por D. R. Lide (CRC, Boca Raton, 1993), pp. 9-19. 
[89] D. B. Laks, C. G. Van de Walle, G. F. Neumark e S. T. Pantelides, Phys. Rev. Lett. 66, 648 (1991).

[90] G. X. Qian, R. M. Martin e D. J. Chadi, Phys. Rev. B 38, 7649 (1988).

[91] N. Chetty e R. M. Martin, Phys. Rev. B 45, 6089 (1992).

[92] Large's Handbook of Chemistry, $12^{a}$ ed., editado por J. A. Dean (McGrawHill, New York, 1979), pp. 9-23 e 9-8; Handbook of Chemistry and Physics, $65^{a}$ ed., editado por R. C. Weast (CRC, Boca Raton, 1984), pp. D-53 e D-68.

[93] R. W. G. Wyckoff, Cristal Structure, Vol. 1, (John Wiley and Sons, 1963).

[94] R. W. G. Wyckoff, Cristal Structure, Vol. 2, (John Wiley and Sons, 1964).

[95] M. E. Sherwin e T. J. Drummond, J. Appl. Phys. 69, 8423 (1991). 